\title{
Design of Transition-Metal Nitride Thin Films for Thermoelectrics
}

\section{Sit Kerdsongpanya}

\author{
ศิษฎ์ เกิดทรงปัญญา
}

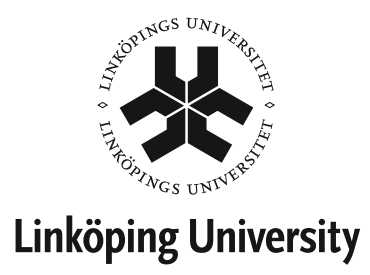

Linköping University

Department of Physics, Chemistry and Biology

Thin Film Physics

SE-581 83 Linköping, Sweden

Linköping 2015 
(C) Sit Kerdsongpanya (ศิษฏ์ เกิดทรงปัญญา)

(C) American Physical Society (Paper)

(C) AIP Publishing LLC (Paper)

ISBN 978-91-7519-067-9

ISSN 0345-7524

Typeset using $\mathrm{ET}_{\mathrm{E}} \mathrm{X}$

Original typesetting format by Olle Hellman

Printed by LiU-Tryck, Linköping 2015 
To those who feel enthusiastic on materials science 



\begin{abstract}
Thermoelectric devices are one of the promising energy harvesting technologies, because of their ability to convert heat (temperature gradient) to electricity by the Seebeck effect. Furthermore, thermoelectric devices can be used for cooling or heating by the inverse effect (Peltier effect). Since this conversion process is clean, with no emission of greenhouse gases during the process, this technology is attractive for recovering waste heat in automobiles or industries into usable electricity. However, the conversion efficiency of such devices is rather low due to fundamental materials limitations manifested through the thermoelectric figure of merit $(Z T)$. Thus, there is high demand on finding materials with high $Z T$ or strategies to improve $Z T$ of materials.
\end{abstract}

In this thesis, I discuss the basics of thermoelectrics and how to improve $Z T$ of materials, including present-day strategies. Based on these ideas, I propose a new class of materials for thermoelectric applications: transition-metal nitrides, mainly ScN, $\mathrm{CrN}$ and their solid solutions. Here, I employed both experimental and theoretical methods to synthesize and study their thermoelectric properties. My study envisages ways for improving the thermoelectric figure of merit of $\mathrm{ScN}$ and possible new materials for thermoelectric applications.

The results of my studies show that $\mathrm{ScN}$ is a promising thermoelectric material since it exhibits high thermoelectric power factor $2.5 \times 10^{-3} \mathrm{Wm}^{-1} \mathrm{~K}^{-2}$ at $800 \mathrm{~K}$, due to low metallic-like electrical resistivity while retained relatively large Seebeck coefficient. My studies on thermal conductivity of ScN also suggest a possibility to control thermal conductivity by tailoring the microstructure of $\mathrm{ScN}$ thin films. Furthermore, my theoretical studies on effects of impurities and stoichiometry on the electronic structure of ScN suggest the possibly to improve ScN $Z T$ by stoichiometry tuning and doping. For $\mathrm{CrN}$ and $\mathrm{Cr}_{1-\mathrm{x}} \mathrm{Sc}_{\mathrm{x}} \mathrm{N}$ solid solution thin films, the results show that the power factor of $\mathrm{CrN}\left(8 \times 10^{-4}\right.$ $\mathrm{Wm}^{-1} \mathrm{~K}^{-2}$ at $770 \mathrm{~K}$ ) can be retained for the solid solution $\mathrm{Cr}_{0.92} \mathrm{Sc}_{0.08} \mathrm{~N}$. Finally, density functional theory was used to enable a systematic predictionbased strategy for optimizing $\mathrm{ScN}$ thermoelectric properties via phase stability of solid solutions. $\mathrm{Sc}_{1-\mathrm{x}} \mathrm{Gd}_{\mathrm{x}} \mathrm{N}$ and $\mathrm{Sc}_{1-\mathrm{x}} \mathrm{Lu}_{\mathrm{x}} \mathrm{N}$ are stabilized as disordered solid solutions, while in the Sc-Nb-N and Sc-Ta-N systems, the inherently layered ternary structures $\mathrm{ScNbN}_{2}$ and $\mathrm{ScTaN}_{2}$ are stable. 


\section{Svensk sammanfattning}

Sedan den industriella revolutionen har fossila bränslen varit vår huvudkälla till energi i motorer för transport, elproduktion och uppvärmning av byggnader. Eftersom mänskligheten och vår teknik växer för varje år som går, fortsätter efterfrågan på fossila bränslen att öka. Med tanke på att fossila bränslen inte är förnybara, riskerar vi att de tar slut. Dessutom är resultatet av denna ständiga förbränning av fossila bränslen generering av växthusgaser, t.ex. kolmonoxid och koldioxid, som orsakar klimatförändringar, som ett ytterligare problem. Således finns det ett ökande behov av nya former av energikällor som kan ersätta fossila bränslen.

För närvarande finns det olika typer av tekniker för förnybar energi som solceller, vätgasteknik (bränsleceller), vindkraftverk, vattenkraft, etc. Ett annat koncept som har studerats är energiåtervinning, vilket innebär att fånga eller lagra spillenergi och förvandla det till användbar energi. Spillenergi är den energi, oftast värmeförluster, som förloras i generatorer, vibrationer från motorer, och så vidare. Ungefär $60 \%$ av den ursprungliga energin avges som spillvärme. Om vi kan återvinna all denna förlust till användbar energi igen, kan vi spara stora mängder bränslen utsläppen av koldioxid kommer att minska.

Med hänsyn till dessa krav, så är termoelektriska komponenter intressanta kandidater. En termoelektriska komponent är tillverkad av material som direkt återvinner värme (en temperaturgradient) till elektrisk energi utan utsläpp av växthusgaser. De kan också kyla genom den omvända processen, när de genererar en temperaturgradient från en pålagd ström. Detta innebär att de kyler utan rörliga delar eller något kylmedel som kan orsaka miljöproblem. Verkningsgraden är emellertid låg, för närvarande 10\% -15\%, dessutom är de flesta av dagens termoelektriska material giftiga. Jag har därför studerat en ny klass av material, övergångsmetallnitrider, som en kandidat för termoelektriska tillämpningar. Övergångsmetallnitrider är kända för sina utmärkta mekaniska egenskaper, de används till exempel som beläggningar på skärverktyg i syfte att förbättra prestanda och livslängd. De uppvisar ocksåolika elektriska egenskaper (metaller, halvledare och supraledare). Min studie är inriktad på att förstå de termoelektriska egenskaperna hos övergångsmetallnitrider, främst skandiumnitrid och kromnitrid. Resultaten visar att båda materialen kan vara bra kandidater för termoelektriska tillämpningar. 


\section{Preface}

This Thesis is a collection of my knowledge and results of my research since July 2010 in the Thin Film Physics Division of the Department of Physics, Chemistry, and Biology (IFM) at Linköping University. This Thesis was initiated as my Master Thesis "Nanolaminate Thermoelectric Thin Films", (LITH-IFM-AEX-10/2296-SE) published 2010 and Licentiate Thesis "Scandium Nitride Thin Films for Thermoelectrics”, (LIU-TEK-LIC-2012:4), published 2012.

My work has primarily been financially supported from the Swedish Research Council (VR) through Grants No. 621-2012-4430 and 621-2009-5258 and the Linköping Center in Nanoscience and technology (CeNano). Additional financial support has been provided from the Linnaeus Strong Research Environment LiLi-NFM, the Swedish Foundation for Strategic Research (Ingvar Carlsson Award and Future Research Leaders 5 to my supervisor), and the European Research Council under the European Community's Seventh Framework Programme (FP/2007-2013) / ERC grant agreement no 335383 (NINA). The calculations were performed using computer resources provided by the Swedish national infrastructure for computing (SNIC) at the National Supercomputer Centre (NSC).

Sit Kerdsongpanya

ศิษฏ์ เกิดทรงปัญญา

Linköping, April 2015 


\section{Acknowledgement}

At the beginning of my Ph.D study, I had a plan that my thesis should be a collection of my knowledge that I have obtain during my Ph.D study. My intention is that my Thesis should be useful to those that have an interest not only in thermoelectric materials development but material science in general. Thus I have put on my best effort to compose this Thesis so it looks as it is today.

This idea cannot have become true if I did not get all the support and contributions from all these people that I have met during my study. Therefore I would like to express my sincere gratitude to the following people:

First of all, I owe my deepest gratitude to my supervisor Per Eklund for the opportunity, trust and belief on that day when I walked into your office and asked for a master project. Thanks you for the freedom and support during my Ph.D study, you always open and listen to my crazy ideas and allow me to grow as a research scientist instead of like feeling a student that walk after someone. And the last thanks to his wise suggestions and great advice that always pour on me. Sorry I would like to thank you more but I am out of words. I hope you feel as I feel.

I would also like to thanks my co-supervisor, Björn Alling, who introduced me how to consider my research problems in theoretical direction, it was fun to work with you. And I would never have thought that I would get chance to work with someone awesome like you. (After I got scared and run away from theoretical project by you). I would like to give my gratitude to my former co-supervisor Gunilla Wingqvist, for teaching me on methodical thinking, how to deal with a research problems, and asking myself a good research question, I would never become like I am today without all of these helps from you. I wish to express my sincere thanks to Lars Hultman, my co-supervisor, for your guidance, support and organization on our division, Thin Film Physics.

I am really grateful to Jens Birch for always giving me nice suggestions and great discussion. The best sentence that I got from you at really early stage still stuck in my heat that is "you have to think it yourself". And I have fun under your lead too, those new changes and organizations.

I would like to thanks all of my co-authors for their contributions on my research papers, especially, Jun Lu for beautiful TEM images, Jens Jensen for ERDA measurements, Ngo Van Nong and Nini Pryds for helping me measure thermoelectric properties, always giving me a lot of good suggestions, and a nice hospitality during my stay at Risø. And the last person, who really influenced me during past two years of my study, Fredrik Eriksson, I would like to give the big thanks to you for the time that you spent on keeping Adam working, so I got enough samples to finish my Ph.D, and the last moment Thesis proofreading from you, it was the biggest help. 
In addition, my study would not go this smooth without helps from these people Thomas, Kalle and Harri a group of people who keep all machines up and running. Inger, Kristin, Malin, and Anette, for an administrative work. Therese for every time I need to pay my conference fee. A big thanks to all of you.

A special thanks to all the people in Thin film physics, Nanostructured materials, Plasma and coatings physics, Nanoscale engineering, and Semiconductor materials division for your direct or indirect help. Thanks to all the member in Agora Materiae graduate school for the fun time that we spent together during past three years, especially, a big thanks to Per Olof Holtz for good mentorship and your effort to make our graduate school being a nice place for all members.

I would like to personally acknowledge all the research funding agencies for giving me an opportunity to enjoy this great experience and be abel to achieve my dream. In addition, thanks for giving us (me and my supervisor) an opportunity to explore on this fascinating research topic. In exchange I have put up all my best effort into my research, so that the results are fulfilled the intention of funding.

A big thanks to Ajan Sukkaneste Tungasmita (Jeed) and Leif Johansson who give such a big opportunity to study at Linköping University without this opportunity all of these great experience of my life time would never happen to me. And I would like to express my biggest gratitude to Ajan Rujikorn Dhanawittayapol without his encourage on that night. I would never come this far.

There is a small group of people that I look forward to see every weekend in front of my computer screen. They are my parents and my little sister in Thailand who makes me laughs every times. Also, they encourage, support, and pray for me every day. I would like to thanks them for everything that they have sacrificed for me up until now.

Finally, thanks to all readers. I am pleased that you show your interest and taking your time to read this Thesis.

Sit Kerdsongpanya

ศิษฏ์ เกิดทรงปัญญา

Linköping, April 2015 


\section{List of included publications}

\section{Paper I}

Anomalously high thermoelectric power factor in epitaxial ScN thin films

Sit Kerdsongpanya, Ngo Van Nong, Nini Pryds, Agnè Žukauskaitė, Jens Jensen, Jens Birch, Jun Lu, Lars Hultman, Gunilla Wingqvist, Per Eklund

Applied Physics Letters 99, 232113 (2011).

\section{Author's contributions :}

I planned the experiments and performed all depositions. I did the X-ray diffraction measurements. I took part in pole figure, transmission electron microscopy, Hall measurements and thermoelectric measurements. I summarized all the results and wrote the article.

\section{Paper II}

Effect of point defects on the electronic density of states of ScN studied by first-principles calculations and implications for thermoelectric properties

Sit Kerdsongpanya, Björn Alling, Per Eklund

Physical Review B 86, 195140 (2012).

Author's contributions :

I planned the study with input from my supervisors, performed all calculations expect the hybrid functional calculation and wrote the article.

\section{Paper III}

Phonon Thermal Conductivity of Scandium Nitride for Thermoelectric Applications from First-Principles Calculations

Sit Kerdsongpanya, Olle Hellman, Bo Sun, Yee Kan Koh, Ngo Van Nong, Jun Lu, Sergei I Simak, Björn Alling, Per Eklund

In manuscript.

Author's contributions :

I planned the theoretical studies together with Olle Hellman and performed all calculations. I planned the experiments and perform all depositions. I did all X-ray diffraction measurements, summarized all data and wrote the article with help from Olle Hellman in the theoretical part. 


\section{Paper IV}

Experimental and Theoretical Investigation of $\mathrm{Cr}_{1-\mathrm{x}} \mathrm{Sc} \mathrm{c}_{\mathrm{x}} \mathrm{N}$ Solid Solutions for Thermoelectric Applications

Sit Kerdsongpanya, Fredrik Eriksson, Jens Jensen, Jun Lu, Bo Sun, Yee Kan Koh, Benjamin Blake, Ngo Van Nong, Björn Alling, Per Eklund

In manuscript.

Author's contributions :

I planned and performed the theoretical studies with input from my supervisors. I planned all experiments and performed all depositions. I did the X-ray diffraction measurements. I took part in thermoelectric measurements. I summarized and interpreted all the results, and wrote the article.

\section{Paper V}

Phase stability of ScN-based solid solutions for thermoelectric applications from first-principles calculations

Sit Kerdsongpanya, Björn Alling, Per Eklund

Journal of Applied Physics 114, 073512 (2013).

\section{Author's contributions :}

I planned the study with input from my supervisors, performed all calculations, analyzed all the data, and wrote the article. 


\section{List of not included publications}

\section{Paper VI}

Phase-stabilization and substrate effects on nucleation and growth of $(\mathrm{Ti}, \mathrm{V})_{\mathrm{n}+1} \mathrm{GeC}_{\mathrm{n}}$ thin films

Sit Kerdsongpanya, Kristina Buchholt, Olof Tengstrand, Jun Lu, Jens Jensen, Lars Hultman, Per Eklund

Journal of Applied Physics 110, 053516 (2011).

\section{Paper VII}

Highly oriented delta- $\mathrm{Bi}_{2} \mathrm{O}_{3}$ thin film stable at room temperature synthesized by reactive magnetron sputtering

Petru Lunca Popa, Stefen Sønderby, Sit Kerdsongpanya, Jun Lu, Nikolaos Bonanos, Per Eklund

Journal of Applied Physics 112, 053516 (2011).

\section{Paper VIII}

Mechanism of Formation of the Thermoelectric Layered Cobaltate $\mathrm{Ca}_{3} \mathrm{Co}_{4} \mathrm{O}_{9}$ by Annealing of $\mathrm{CaO}-\mathrm{CoO}$ Thin Films

Biplab Paul, Jeremy L. Schroeder, Sit Kerdsongpanya, Ngo Van Nong, Norbert

Schell, Daniel Ostach, Jun Lu, Jens Birch, Per Eklund

Advanced Electronic Materials 1, 140022 (2015). 


\section{Table of Contents}

1 Introduction 3

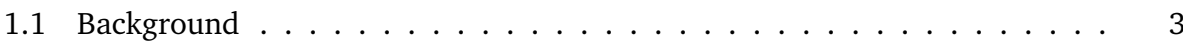

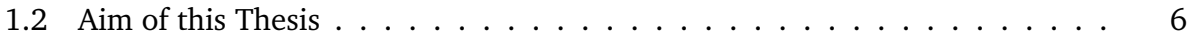

1.3 Outline of this Thesis . . . . . . . . . . . . . 7

2 Fundamentals of thermoelectricity 9

2.1 Thermoelectric effects . . . . . . . . . . . . . . . . . . 9 9

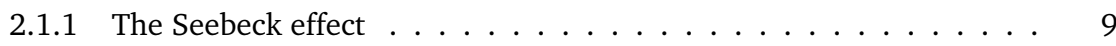

2.1 .2 The Peltier effect . . . . . . . . . . . . . . . . . . 10

2.1 .3 The Thomson effect . . . . . . . . . . . . . . . 11

2.1.4 Thermoelectric mechanisms . . . . . . . . . . . . . . . 11

2.2 Thermoelectric efficiency . . . . . . . . . . . . . . . . . . 15

2.2.1 Carnot's theorem . . . . . . . . . . . . . . . . 15

2.2.2 Coefficient of performance, Efficiency of heat engine, and Thermoelectric figure of merit . . . . . . . . . . . . 16

2.2.2.1 Thermoelectric refrigeration and coefficient of performance 16

2.2.2.2 Thermoelectric generation and efficiency of generator . . 18

2.2.2.3 Thermoelectric Figure of Merit, geometrical considerations 19

2.2.3 Basic consideration of improving thermoelectric figure of merit . . . 19

3 Thermoelectric figure of merit 23

3.1 Lattice dynamics . . . . . . . . . . . . . . . . . . . . . . . . . . . . . . . 3

3.2 Lattice thermal conductivity . . . . . . . . . . . . . . . 30

3.3 Seebeck coefficient, electrical resistivity and electron thermal conductivity . 32

3.4 Maximization of thermoelectric figure of merit . . . . . . . . . . . . 35

3.4.1 Minimizing lattice thermal conductivity . . . . . . . . . . . . . . 35

3.4.1.1 Phonon-phonon scattering . . . . . . . . . . 36

3.4.1.2 Phonon scattering by impurities or alloying atoms . . . . . 38

3.4.1.3 Phonon scattering by grain boundary scattering . . . . . . 41

3.4.1.4 Nanostructure all-scale hierarchical architecturing . . . . . 42

3.4.2 Improving Seebeck coefficient and electrical conductivity . . . . . . 44

3.4.2.1 The "best" electronic band structure for thermoelectrics . . 44

3.4.2.2 The materials parameter, B . . . . . . . . . . 46

3.4.2.3 Optimum band gap of thermoelectric materials . . . . . 51

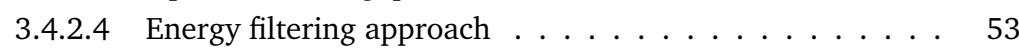

3.4.2.5 Modulation doping . . . . . . . . . . . . . 54

3.4.2.6 Reducing the electronic thermal conductivity . . . . . 56

3.5 Review of scandium nitride $(\mathrm{ScN}) \ldots \ldots \ldots \ldots$ 
3.6 Review of chromium nitride $(\mathrm{CrN}) \ldots \ldots \ldots \ldots$. . . . . . . . . 61

4 Theoretical calculations $\quad 67$

4.1 Ab-initio calculations . . . . . . . . . . . . . . . . . 67

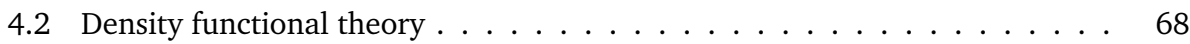

4.3 Khon-Sham equation . . . . . . . . . . . . . . . 71

4.4 Exchange and correlation functional approximations . . . . . . . . . . . 74

4.4.1 Local density approximation . . . . . . . . . . . . . . . . . 74

4.4.2 Generalized gradient approximations . . . . . . . . . . . . . 75

4.4.3 Hubbard-corrected ("+U") approximation $(\mathrm{DFT}+\mathrm{U}) \ldots \ldots 76$

4.4.4 Hybrid functionals . . . . . . . . . . . . . . . . . 79

4.4.5 Pseudopotentials and Projector augmented wave . . . . . . . . . . 80

4.5 Phase stability . . . . . . . . . . . . . . . . 82

4.5.1 Phase stability of solid solution and decomposition processes . . . . 84

4.6 Phase Stability Calculations . . . . . . . . . . . . . . . 86

4.6.1 Configurational Modeling of solid solution . . . . . . . . . . . 88

4.7 Molecular Dynamics _. . . . . . . . . . . . . . . . . . . . . . . . . . . . . . .

4.7.1 Basic molecular dynamics formalism . . . . . . . . . . . 92

4.7.2 Time integration algorithm . . . . . . . . . . . . . . . 97

4.7.3 Thermodynamical averages in molecular dynamics . . . . . . . . . . . 98

4.7.4 Thermostat . . . . . . . . . . . . . . . . . 100

4.7.5 Free energy determination in molecular dynamics . . . . . . . . . 104

4.7.6 Basic molecular dynamic routine . . . . . . . . . . . . 106

4.8 Force constants calculations . . . . . . . . . . . . . . . . 108

4.8.1 One dimensional anharmonic potential . . . . . . . . . . . . 109

4.8.2 Temperature Dependent Effective Potential Method (TDEP) . . . . . 112

4.8.3 The Extension of Temperature Dependent Effective Potential Method to the periodic crystal lattice . . . . . . . . . . . . . . . . . . 114

4.8 .4 Free energy calculations . . . . . . . . . . . . . . . 117

4.8.5 Lattice thermal conductivity calculations . . . . . . . . . . . . . . . 119

5 Deposition processes $\quad 123$

5.1 The physics of sputtering . . . . . . . . . . . . . . . . . . 123

5.1 .1 Plasma. . . . . . . . . . . . . . . . . . . . . 124

5.1.2 The plasma, floating, and bias potentials, potential distribution, and sheath . . . . . . . . . . . . . . . . . 126

5.1 .3 Transport of sputtered species . . . . . . . . . . . . . 126

5.1 .4 Effects at the substrate . . . . . . . . . . . . . . . . . . . . . . . . . . 127

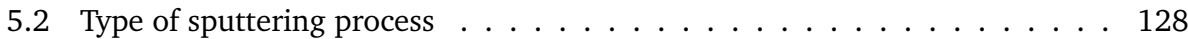

5.2 .1 DC magnetron sputtering . . . . . . . . . . . . . . 128

5.3 Reactive sputtering . . . . . . . . . . . . . . . 130 
6 Characterization techniques 133

6.1 Structure characterization . . . . . . . . . . . . . 133

6.1 .1 X-ray Diffraction $(\mathrm{XRD}) \ldots \ldots \ldots \ldots 133$

$6.1 .1 .1 \quad \theta-2 \theta \operatorname{scan} \ldots \ldots \ldots \ldots 134$

6.1.1.2 $\omega$ scan or rocking curve measurement . . . . . . . . 135

6.1.1.3 Pole figure measurement . . . . . . . . . . . 136

6.1 .2 Scanning Electron Microscope (SEM) . . . . . . . . . . . . . 137

6.1.3 Transmission Electron Microscope (TEM) . . . . . . . . . . . . . . 137

6.2 Compositional characterization . . . . . . . . . . . . . . . . 140

6.2.1 Energy-Dispersive X-ray Spectroscopy (EDX or EDS) . . . . . . . . . 140

6.2.2 Elastic Recoil Detection Analysis (ERDA) . . . . . . . . . . . . . 140

6.3 Electrical characterization . . . . . . . . . . . . . . . . . 141

6.3 .1 Resistivity measurement . . . . . . . . . . . . . . . . . . 141

6.3.2 Bulk carrier concentration and Hall mobility measurement . . . . . 142

6.3.3 Seebeck coefficient measurement . . . . . . . . . . . . . . . . . . . 144

6.3.4 Time-Domain Thermoreflectance (TDTR) . . . . . . . . . . . . . 146

$\begin{array}{lll}7 & \text { Summary and my contributions to the field } & 153\end{array}$

$\begin{array}{lr}\text { Bibliography } & 159\end{array}$

$\begin{array}{ll}\text { Paper I } & 181\end{array}$

Anomalously high thermoelectric power factor in epitaxial ScN thin films

Paper II

Effect of point defects on the electronic density of states of ScN studied by firstprinciples calculations and implications for thermoelectric properties

Paper III

Phonon Thermal Conductivity of Scandium Nitride for Thermoelectric Applications from First-Principles Calculations

Paper IV

Experimental and Theoretical Investigation of $C r_{1-\mathbf{x}} S c_{\mathbf{x}} N$ Solid Solutions for Thermoelectric Applications

\section{Paper V}

Phase stability of ScN-based solid solutions for thermoelectric applications from firstprinciples calculations 



\section{List of figures}

2.1 Illustration of Seebeck effect circuit . . . . . . . . . . . . 9

2.2 Illustration of Peltier effect circuit . . . . . . . . . . . . . 10

2.3 Thermoelectric devices . . . . . . . . . . . . . . . . . 12

2.4 Energy band diagrams for a thermoelectric power generation mode 13

2.5 Energy band diagrams for a thermoelectric refrigerator mode . . 14

2.6 Carnot's cycle in P-V diagram of a heat engine . . . . . . . 15

4.1 The schematic plot of Gibbs free energy versus material density . $\quad 82$

4.2 The schematic plot of the mixing Gibbs free energy with the molar fraction of solid solution $\mathrm{A}_{1-\mathrm{x}} \mathrm{B}_{\mathrm{x}}$ and relate phase diagram . . . 84

4.3 One dimensional oscillators . . . . . . . . . . . . . . . . 89

4.4 Comparison of thermostats . . . . . . . . . . . . . . . . 102

4.5 The molecular dynamics flow chart . . . . . . . . . . 106

4.6 Comparison of the temperature dependent effective potential method (TDEP) and the harmonic approximation in one dimensional potential . . . . . . . . . . . . . . . . . 110

4.7 Higher order expansion of one dimensional potential . . . . . 111

5.1 Sputtering setup and process schematics drawing . . . . . . . 124

5.2 Potential distribution in DC glow discharge . . . . . . . . . . 126

5.3 The magnetron arrangements . . . . . . . . . . . . . . . . . . . 129

5.4 The reactive sputtering hysteresis behavior . . . . . . . . . 130

6.1 Illustration of a $\theta-2 \theta$ scan . . . . . . . . . . . . . . . . . 135

6.2 Illustration of an $\omega$ scan $\ldots \ldots \ldots \ldots$

6.3 Illustration of a pole figure measurement . . . . . . . . . . . 136

6.4 Four point probe setup . . . . . . . . . . . . . . . . . 141

6.5 Hall effect schematic . . . . . . . . . . . . . . . . . . . . . 142

6.6 Schematic of resistivity measurement van der Pauw configuration 143

6.7 Schematic of Hall measurement van der Pauw configuration . . 143

6.8 Probe configuration for Seebeck measurement . . . . . . . . . . 144

6.9 The time-domain thermoreflectance on aluminum thin film . . . 148

6.10 The schematic drawing of the time-domain thermoreflectance setup 150 

人は何かの犠牲なしに何も得ることはできない。

何かを得るためには同等な代価が必要になる。

それが鍊金術における等価交換の原則だ。

そのころ僕らはそれが世界の真実だと信じてい

た。

Hito wa nanika no gisei nashi ni nani mo eru koto wa dekinai. Nanika o eru tameni wa dotona daika ga hitsuyo ni naru. Sore ga renkinjutsu ni okeru toka kokan no gensokuda. Sono ko ro bokura wa sore ga sekai no shinjitsuda to shinjite ita.

Humankind cannot gain anything without first giving something in return. To obtain, something of equal value must be lost. This is Alchemy's First Law of Equivalent Exchange. In those days, we really believed that to be the world's one, and only truth.

Said by Alphonse Elric from the episodes opening scene of Fullmetal Alchemist by Hiromu Arakawa, Original Creator 



\section{Chapter 1}

\section{Introduction}

"The most important thing about global warming is this. Whether humans are responsible for the bulk of climate change is going to be left to the scientists, but it is all of our responsibility to leave this planet in better shape for the future generations than we found it."

Mike Huckabee, former governor of Arkansas

\subsection{Background}

Today, fossil fuels are the main sources of energy for transportation, electricity, and heating or cooling your building. Consequently the demand for fossil fuels is increasing every year as reported by the International Energy Agency (IEA). In addition, they predicted that the demand will keep increase by about $37 \%$ at the end of 2040. ${ }^{1}$ Moreover, the result of continuously burning these fossil fuels is the generation of greenhouse gases causing global warming or climate change. Thus, humanity is facing a serious energy and climate issue, as results there is an increasing pressure on finding new technologies that can help us to solve these problems. One is finding new sustainable, clean, high efficient energy sources. Thus, solar thermal, solar cells, hydrogen technology (fuel cells), wind turbines, hydroelectric gravity dam, tidal wave power station, etc. have been developed for that purpose. Also, enhancing the efficiency on use of energy is contribution to solve the energy problems. This concept has shown in the 20-2020 climate and energy package that official policies announced by European Union (EU) as part of the Europe 2020 strategy with the objective to reduce the greenhouse gas by $80 \%$ within $2050 .{ }^{2}$ The details of the 20-20-20 climate and energy package are (i) a cut in greenhouse gas emission by $20 \%$ compared to 1990 emissions, (ii) Increasing the energy production from the renewable energy sources to $20 \%$, and the last (iii) Improvement in the EU's energy 
efficiency by $20 \%$. And recently the commission has expanded to 2030 in "the 2030 framework for climate and energy policies" as a first step of longer term plan with the higher aim of greenhouse gas reduction and increase of share in production of energy by renewable energy.

Thermoelectric technology can contribute to achieve those three demands. Thermoelectric devices convert heat (a temperature gradient) into electrical energy and perform cooling or heating by reverse process without moving parts and releasing any emission of greenhouse gases, because thermoelectrics are solid state devices. Thus the energy harvested by thermoelectric devices is renewable and clean. The source of temperature gradient can be solar energy, or waste heat from household, automobile, or industry. However, the efficiency of cooling and generating electrical energy is fairly low, ${ }^{3-7}$ since the efficiency of thermoelectric devices is limited by the thermoelectric figure of merit $(Z T)$ of the materials as well as their design. Currently we can obtain materials with $Z T \sim 1$ yielding a device efficiency of $10-20 \%$ of Carnot efficiency. $^{3}$ This should be compared to the $40-50 \%$ of heat engines.

Vining commented that we needed materials that have a $Z T$ of 20 to be able to replace current heat engines but that this number seems unlikely. ${ }^{8}$ Nevertheless, thermoelectric devices can play a role in increasing the efficiency of current technology by reducing the energy consumption or increasing, since about $60 \%$ of energy which is produced in the heat engine is lost as waste heat during the energy conversion process. For example, in the automobiles $80 \%$ of initial energy that is conversed from burning the petroleum in combustion process is loss. Only about $20 \%$ that is used to drive the automobiles. Among $80 \%$ of initial energy loss about $60 \%$ is a wasted heat i.e., $30 \%$ in exhaust heat and $30 \%$ in radiator heat, and the last $20 \%$ are friction and alternator. ${ }^{9}$ Nowadays, automobiles utilize this $5 \%$ of friction loss during breaks in terms of hybrid engine. If we use thermoelectric devices to capture or harvest these waste energies and convert into useable electricity, petroleum and coal consumption will be reduced. There is a current development on use of thermoelectric devices as electrical generator for vehicles by collecting waste energy from exhaust gas ${ }^{10,11}$ which corresponds to about $30 \%$ of energy produced by petroleum loss in combustion process. This waste heat recovery 
concept can be implemented in industry as well; thermoelectric devices with $\sim 1 \%$ efficiency with lifetimes longer than 5 years, are economically sustainable. ${ }^{12}$ Another important application of thermoelectric is improving efficiency of another renewable energy production technology.

Recently there is research on coupling thermoelectric device to photovoltaics (solar cell) in order to improve photovoltaic efficiency, showing that with $15{ }^{\circ} \mathrm{C}$ of temperature gradient produced from photovoltaic. This coupling can improve the photovoltaic efficiency from $12.5 \%$ to $16.3 \%$ of conversion efficiency. ${ }^{13}$ Furthermore, the authors also reported on the possibility to reduce photovoltaic degradation during operation due to cooling effect on thermoelectric device. The solar thermal technology also becomes an interesting renewable energy production as shown $7.7 \%$ increase in energy production from 2011 and 2012. ${ }^{2}$ By utilizing thermoelectrics, the efficiency of energy production by solar thermal devices should be improved. These considerations show that thermoelectrics is a promising technologyfor mitigating the energy crisis and climate change as energy production or improvement of the energy production efficiency technology without greenhouse gas production.

Today, thermoelectric devices are commonly used in the field of cooling/heating or sensing. Cooling and heating applications are easy to realize, despite the low $Z T$ of the devices, since we only need to make sure that we supply high enough current into the device to reach the needed cooling or heating temperature. Thus there are many products in the market ${ }^{14}$ e.g., picnic or wine coolers and niche applications like semiconductor laser cooling. ${ }^{15}$ For sensor applications, are also remarkably easy for example in temperature or water condensing sensor, ${ }^{16}$ since they do not require high conversion efficiency as long as the device gives a signal. On the other hand, the conversion efficiency of current thermoelectric device is a big issue for electrical generation applications. It is rare to see commercialized products for thermoelectric generators. Most of them are still under research e.g., waste heat recovery from exhaust gas in automobile, ${ }^{10,11}$ waste heat recovery in jet engine, ${ }^{11}$ or solar thermoelectric generator. ${ }^{17,18}$ The real application that has been implemented is in space missions by NASA in the form radioisotopic decay thermoelectric generators (RTGs). ${ }^{19,20}$ 
Despite the interesting application of thermoelectric device, the efficiency of the device is still too low for advanced application because low thermoelectric figure of merit $(Z T)$ materials are used. Thus, there is a demand and effort on improvement of thermoelectric materials with a goal of $Z T \sim 4$. However, the improvement of $Z T$ is not trivial, because the fundamental parameters that determine $Z T$ are interrelated yielding non-improvement of $Z T$. Many attempts have been made to maximize $Z T$ resulting in $Z T$ of $1-2 .{ }^{4,7,21}$ This shows that the maximizing thermoelectric figure of merit a fascinating research problem.

\subsection{Aim of this Thesis}

The scope of my research in this Thesis is to investigate and understand thermoelectric phenomena in a novel class of thermoelectric materials: transition-metal nitrides, mainly scandium nitride $(\mathrm{ScN})$, chromium nitride $(\mathrm{CrN})$, and their solid solution. There are not many studies focus on thermoelectric properties in transition-metal nitrides, despite the fact that they exhibit excellent mechanical properties, e.g., high hardness and elastic modulus, chemically inert, high oxidation resistance, these are required for harsh environment applications such as heat recovery in automobiles, jet engine, or industrial system. In term of electrical properties, Transition-metal nitride have wide range electrical properties which vary from metallic to semiconducting depending on type of transition metal element and their stoichiometry meaning that a possibility to modify electronic properties for high $Z T$. In addition they can withstand large temperature gradient without degradation or oxidation at mid-to-high temperature regime (300-800 K) which is an important requirement for thermoelectric application. Because the conversion efficiency is proportional to both temperature gradient and $Z T$. I have used experimental and theoretical techniques to study the relation between materials structure and orientation, chemical composition, and their electronic structure with transport properties. The knowledge that obtained from these studies will be used to suggest the possible strategies on improving $Z T$ in general. 


\subsection{Outline of this Thesis}

I would like to compose this Thesis as the collection of my knowledge that I have gained since I start my Ph.D. Apart from this reason, I would like to make my Thesis useful to whom that has interest in material science, especially in thermoelectric material development. This Thesis starts with introduction of basic knowledge of thermoelectric phenomena and the efficiency of thermoelectric device and material in Chapters 2. The thermoelectric figure of merit, which is a key parameter is also introduced, discussed in light of basic transport parameters and it is suggested how to improve thermoelectric properties based on reviewing of current thermoelectric materials in Chapter 3. Later, Chapter 4-6 will describe the theoretical methods, deposition technique, and characterization techniques that are used in this Thesis. Finally the summary of important findings during my study is in Chapter 7. 



\section{Chapter 2}

\section{Fundamentals of thermoelectricity}

"Nothing splendid was ever created in cold blood. Heat is required to forge anything. Every great accomplishment is the story of a flaming heart."

Arnold H. Glasow, former General of the Air

Force

In this chapter, we will discuss three important thermoelectric effects, i.e., Seebeck effect, Peltier effect, and Thomson effect (or Kelvin effect). Then we discuss on thermoelectric mechanism and efficiency of thermoelectric devices.

\subsection{Thermoelectric effects}

\subsubsection{The Seebeck effect}

Upon attempting to understand the magnetization of the earth, Thomas Johann Seebeck reported his discovery of the thermoelectric effect in 1821-23. ${ }^{22-24}$ He proposed that the magnetism of two different metals ( $\mathrm{Bi}$ and $\mathrm{Cu}$ ) was generated when one of the junctions were heated. Seebeck called this effect "thermomagnetism". Later in 1823, Hans Christian Ørsted showed that the temperature gradient generates electricity rather than magnetism as Seebeck proposed. ${ }^{25}$ The debate between $\emptyset$ rsted and Seebeck led to an important consequence in discovery conversion of magnetic field into electric field, i.e., Ampere's law. In contrast to Ampere's law, the phenomenon that Seebeck found is an electric current that is driven in a closed circuit due to an electromotive force (EMF) or voltage that is generated in a pair of dissimilar metals at a given temperature gradient. This effect is called Seebeck effect and illustrated the simple circuit in Figure 2.1. 
This effect gives definition of the "Seebeck coefficient" $(S)$ (also called thermoelectric power or thermopower) can be defined as the open circuit voltage that is generated by a temperature gradient.

$$
S_{\mathrm{AB}}=S_{\mathrm{B}}-S_{\mathrm{A}}=\frac{\Delta V_{\mathrm{AB}}^{o c}}{T_{\mathrm{H}}-T_{\mathrm{C}}}=\frac{\Delta V_{\mathrm{AB}}^{o c}}{\Delta T},
$$

where, $S_{\mathrm{AB}}=S_{\mathrm{B}}-S_{\mathrm{A}}$ is the difference in Seebeck coefficient of material $A$ and $B$ (usually in the unit of $\mu \mathrm{VK}^{-1}$ ), $T_{\mathrm{H}}$ and $T_{\mathrm{C}}$ are the temperature of hot end and cold end, respectively, and $V_{\mathrm{AB}}^{o c}$ is the open circuit voltage that is generated by a temperature gradient. Later in 1851, Gustav Magnus ${ }^{26,27}$ found that the Seebeck voltage is constant over the distribution of temperature along the metals between junctions meaning that the Seebeck coefficient is the thermodynamic state function. This discovery led to the development of thermocouple which can be used to measure temperature. To measure a temperature difference directly or an absolute temperature by setting one end to a known temperature, the thermoelectric voltage which is produced by heating is scaled up with a pair of dissimilar metals with known Seebeck coefficients, allowing for temperature to be determined.

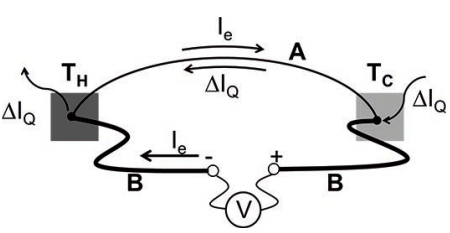

FIGURE 2.2: A Peltier effect circuit consists of semiconductor with two metal contacts, the electron current flow in from left to right which generate the heat current flow from right to left. The $T_{H}$ and $T_{C}$ are the temperature of hot end and cold end, respectively.

\subsubsection{The Peltier effect}

In 1834, the second thermoelectric effect was discovered by JeanCharles Peltier; it is called the Peltier effect. ${ }^{22,28}$ He showed that when electrical current flowed into dissimilar metals, the heating effect occurred when reverse electrical current was applied (see Figure 2.2). Similar to Seebeck, Peltier wrongly related his discovery to the Joule heating by claiming that there were no Joule heating when the low current pass though the circuit. Later in 1838, Heinrich Friedrich Emil Lenz, proved the discovery of Peltier and showed that the heating or cooling effect depends on the direction of applied current. ${ }^{29}$ Thus Lenz independently confirmed the effect of Joule heating in 1842, because Joule heating is independent of the direction of the current. The Peltier coefficient of two dissimilar materials can be define by the ratio between the heat current $\left(I_{\mathrm{Q}}\right)$ and the (electron) current density $(I)$,

$$
\left(\Pi_{\mathrm{B}}-\Pi_{\mathrm{A}}\right)=\Pi_{\mathrm{AB}}=-\frac{\Delta I_{\mathrm{Q}}}{I},
$$


where $\Pi_{\mathrm{AB}}=\Pi_{\mathrm{B}}-\Pi_{\mathrm{A}}$ is the differential Peltier coefficient of materials $A$ and $B$ in units of $\left(\mathrm{WA}^{-1}\right.$ ). The Peltier effect is quite difficult to measure experimentally due to Joule heating, which occurs when current is passed though metals due to electrons scattering.

\subsubsection{The Thomson effect}

The relation between the Seebeck and Peltier effects was described by William Thomson (later Lord Kelvin) in 1855, who predicted using the laws of thermodynamics and studied experimentally the rate of cooling when applying current in a single conductor having a temperature gradient. This effect is called Thomson effect, ${ }^{22}$ which is the third thermoelectric effect. The heating or cooling $(q)$ is

$$
q=\beta I \Delta T
$$

where $\beta$ is the Thomson coefficient of material in units $\left(\mathrm{VK}^{-1}\right)$, $I$ is the current which passes through the materials, and $\Delta T$ is the temperature different. The heating or cooling effect depends on electrical discharge of material which gives positive Thomson effect $(+\beta)$ or negative Thomson effect $(-\beta)$. The Thomson effect yields the relation between Seebeck and Peltier coefficient

$$
\Pi_{\mathrm{AB}}=S_{\mathrm{AB}} I
$$

and

$$
\frac{\beta_{\mathrm{AB}}}{T}=\frac{d S_{\mathrm{AB}}}{d T} .
$$

Both are useful for calculating the Seebeck and Peltier coefficient, since we cannot measure the absolute Seebeck and Peltier coefficient directly. Thomson coefficient can be measured directly.

\subsubsection{Thermoelectric mechanisms}

Now we will discuss all these thermoelectric effects in light of semiconductor physics. ${ }^{30}$ Figure 2.3 shows schematic drawings of simple thermoelectric devices in both power generator (left) and refrigerator (right) mode. Due to the difference in the chemical potential (Fermi energy), the charge carriers (electrons and holes) have to redistribute until their chemical potentials are equal in the 
FIGURE 2.3: Schematic drawing of (a) thermoelectric device in power generation mode and $(b)$ in refrigeration mode. The devices are made from n-type (electron conduction) and p-type (hole conduction) thermoelectric materials which are connected in a series with metal interconnect between them. The devices are placed parallel with the heat flow with heat source (or active cooling) and sink.

entire device. This effect results in band bending and formation of potential barriers at all the junction because of the difference in their conduction and valence bands. ${ }^{31}$ The corresponding energy band diagram for the thermoelectric generation mode can be seen in Figure 2.4. Since the device is subjected into the temperature gradient, this temperature gradient will give thermal energy into charge carrier (both holes and electrons) at the hot junction between semiconductor and metals interconnection, thus the charge carriers can now diffuse across the potential barrier. After absorbing thermal energy, the charge carriers at the hot end (hot carriers) will have higher energy than those at the cold end (cold carriers) leading to difference in momentum between these two type of charge carriers. Therefore, hot carriers can diffuse to the cold end faster than cold carriers diffuse back to the hot end resulting in a net current from hot to cold end. Once the hot carriers reach the cold end, they will relax to the metal interconnect by releasing heat.

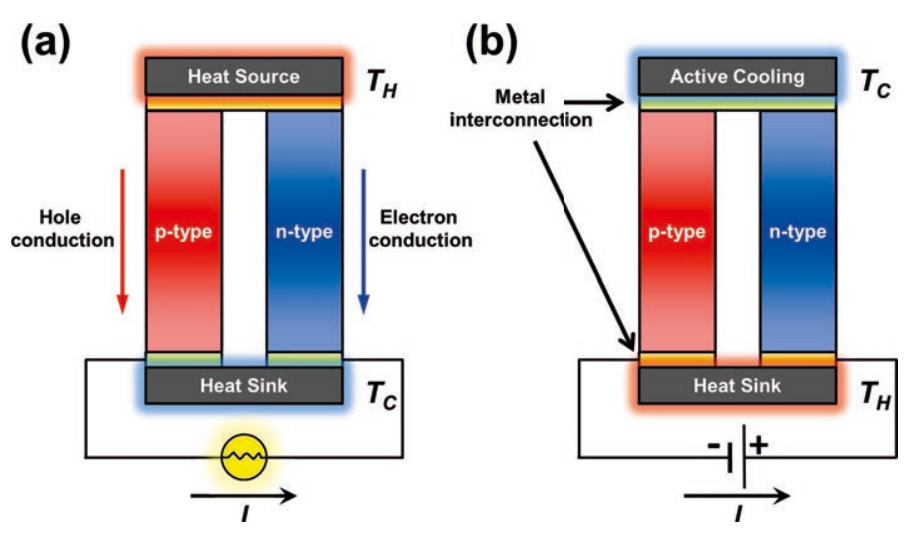

In addition to the higher temperature at hot end, the Fermi-Dirac distribution of charge carriers is also more diverse (soft) at the hot end than the cold end. This means that there is higher hot carrier concentration than the cold carriers. Furthermore, the thermal energy at hot end can generate more carriers in the semiconductor via thermal ionization donors and acceptor in n-type and p-type semiconductor, respectively. This results in a difference in the carrier concentration (concentration gradient) across the semiconductor legs and further energy band distortion. The results of these two effects combine and give the concentration gradient across both n-type and p-type semiconductor where holes and electrons will diffuse from high concentration to the 
low concentration (hot to cold end) as electrons and holes moves from higher energy states to lower one.

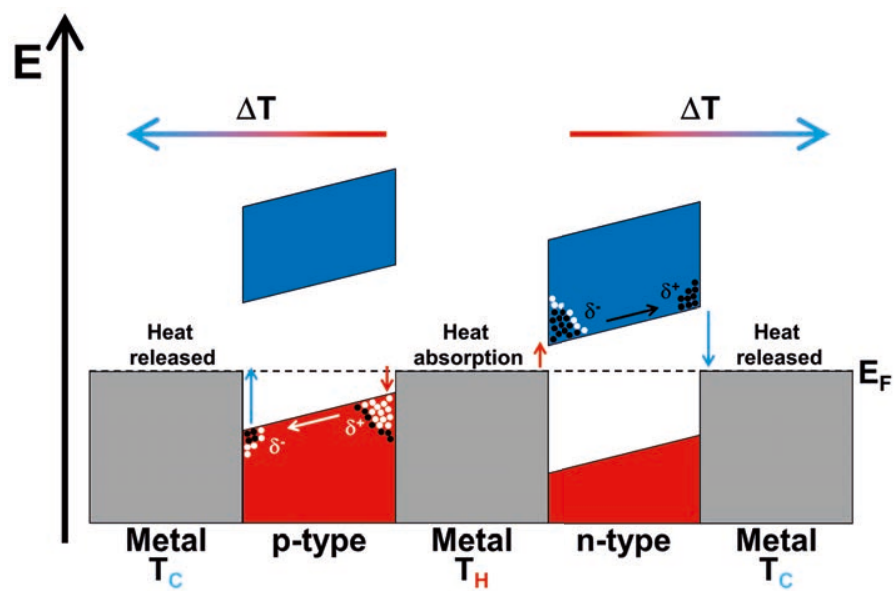

If these three processes continue, more electrons and holes are generated and diffuse away to the cold end. The electric field start to develop across semiconductor due to the left behind ionized donors and acceptors yielding the back flow of charge carriers (current). In the end the steady-state equilibrium of diffusion current that driven by the concentration gradient and back flow of current driven by the electric field is reached (see Figure 2.4). Thus, these mechanisms lead to the development of a voltage difference between hot and cold side which is called "thermoelectric voltage". And this is the explanation of "Seebeck effect".

Now, let us switch to consider the thermoelectric device in the refrigeration mode (see Figure 2.3(b)). In refrigeration mode, we apply current into the device instead of temperature gradient, meaning that we inject electrons into the device. Then those electrons will be drifted through the semiconductor and stop at metal-semiconductor junction which electrons cannot penetrate through due to the potential barrier. The electrons have to absorb thermal energy from the surrounding in order to pass the potential barrier and release the thermal energy when they relax at the end point of both materials, see Figure 2.5 for the energy band diagram in the refrigeration mode. This will cause a temperature drop at the junction and increasing temperature at the end point of the material, resulting in cooling effect at the junction of metal-semiconductor and heating effect then another end.
FIGURE 2.4: Schematic drawing of corresponding energy band diagrams for a thermoelectric power generation device in a temperature gradient at steady-state equilibrium. Here the gray area denotes as the conduction band of metal interconnections. Since they are metal, so there is not energy gap, unlike the $n$-type and p-type semiconductor, there is energy gap separates the valence band (red area) and conduction band (blue area). The white and black dot represents holes (ionized donors) and electrons (ionized acceptor), respectively. 
FIGURE 2.5: Schematic drawing of corresponding energy band diagrams for a thermoelectric cooling device after the electric current applied at steady-state equilibrium. Here the current is injected from left to right. Here, we use the same color scheme as in the power generation mode. Note that the effect of cooling or heating changes with the direction of electron current.
These cooling and heating effects will generate the temperature gradient across the device. This temperature gradient will leads to Seebeck effect which then brings the system to steady-state equilibrium. This is the explanation of "Peltier effect".

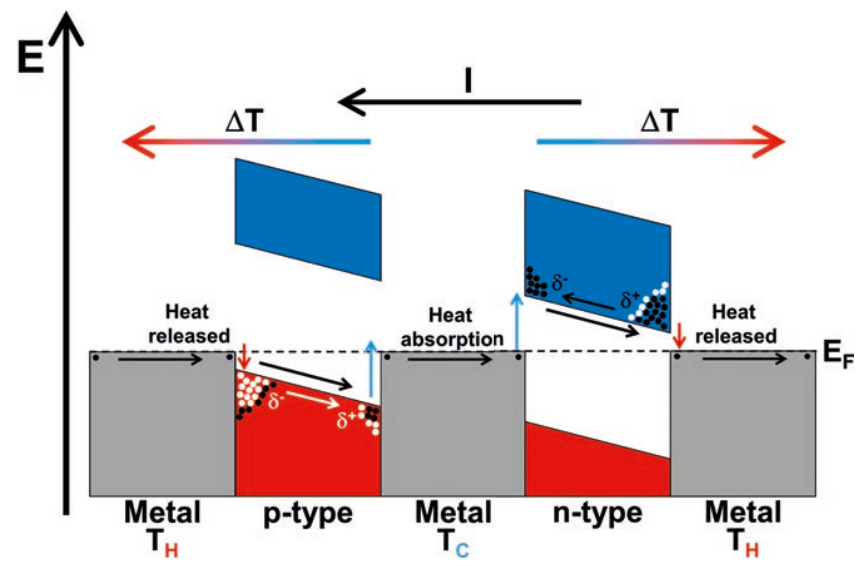

These discussions show that we can utilize Seebeck effect for generating electricity, as long as we supply heat onto one junction of the device and Peltier effect for cooling and heating without cooling agent, meaning that no greenhouse gas is used during cooling or heating process. These applications are interesting for the current situation where high demand on clean and renewable energy and green house gas reduction. In next section we will discuss on the efficiency of thermoelectric device. 


\subsection{Thermoelectric efficiency}

Thermoelectric devices can be considered as heat engines or heat pumps. This section is going to show how the efficiency of thermoelectric devices can be obtained from thermodynamics, and the relation of the thermoelectric figure of merit which is a number that determines the performance of thermoelectric devices.

\subsubsection{Carnot's theorem}

Since thermoelectric devices are a type of heat engines or heat pumps, consider the possible maximum efficiency. The ideally highest efficiency of heat engines and heat pumps was proposed by Nicolas Léonard Sadi Carnot in 1824 from basic ideas in thermodynamics. ${ }^{32}$ Carnot's theorem states that

"No engine operating between two reservoirs can be more efficient than a Carnot's engine operating between those same two reservoirs"

This theorem leads to the conclusion that the highest efficiency engine must work in a reversible cycle between hot and cold reservoirs without any loss, i.e., a cycle must include isothermal and adiabatic processes. This cycle is called Carnot cycle. The Carnot cycle of a heat engine is shown in Figure 2.6(a). The definition of a heat engine efficiency ${ }^{32}(\eta)$ for the heat engines is

$$
\eta=\frac{|\Delta W|}{\left|\Delta Q_{\mathrm{H}}\right|}
$$

where $\eta$ is the efficiency of generator, $\Delta W$ is the energy output from heat engine, and $\Delta Q_{\mathrm{H}}$ is the heat absorbed by the heat engine. The highest efficiency of a heat engine is the Carnot efficiency

$$
\eta=1-\frac{Q_{\mathrm{C}}}{Q_{\mathrm{H}}}=\frac{T_{\mathrm{H}}-T_{\mathrm{C}}}{T_{\mathrm{H}}} .
$$

The reverse Carnot cycle gives the coefficient of performance ${ }^{32}$ (COP) for heat pumps:

$$
\eta=\frac{\left|\Delta Q_{\mathrm{H}}\right|}{|\Delta W|},
$$

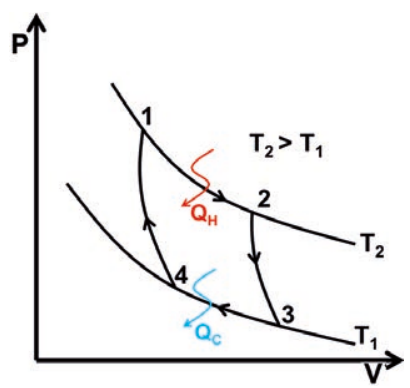

FIGURE 2.6: The Carnot's cycle in P-V diagram which include two isothermal line connect with two adiabatic lines. 
where $\Delta Q_{\mathrm{H}}$ is the net heat moved from cold side to hot side (cooling power), $\Delta W$ is the net energy consumed. The Carnot coefficient of performance is

$$
\eta=\frac{1}{1-\frac{Q_{\mathrm{C}}}{Q_{\mathrm{H}}}}=\frac{T_{\mathrm{C}}}{T_{\mathrm{H}}-T_{\mathrm{C}}} .
$$

\subsubsection{Coefficient of performance, Efficiency of heat engine, and Thermoelectric figure of merit}

The thermoelectric mechanisms discussed in section (2.1.4) leads us to consider how they can work as power generator (heat engine) or refrigerator (heat pump) utilizing the Seebeck or the Peltier process, respectively. These processes are in principle thermodynamically reversible. Unfortunately, there are also irreversible processes, i.e., Joule heating (due to electrical resistance in device) and thermal conduction. The actual efficiencies of thermoelectric refrigeration and generation are determined by applied thermodynamic concepts which give the relation to thermoelectric figure of merit $\left(Z T_{m}\right),{ }^{22,33,34}$ where $T_{m}$ is the average temperature over the device, $Z$ is dependent on the Seebeck coefficient $\left(S_{\mathrm{np}}\right)$, the total series resistance of the device $(R)$, and the total thermal conduction $(K)$ of the device. ${ }^{I}$ This section will show how we can determine this thermoelectric figure of merit.

\subsubsection{Thermoelectric refrigeration and coefficient of performance}

The coefficient of performance, COP of thermoelectrics in the refrigeration mode can be calculated by considering simple system as shown in Figure 2.3(b), thus the net absorbed heat is given in

$$
Q=S_{\mathrm{np}} I T_{\mathrm{C}}-K \Delta T-\frac{1}{2} I^{2} R
$$

where $S_{\mathrm{np}}$ is the difference in Seebeck coefficient from each thermoelectric material $\left(S_{\mathrm{np}}=S_{\mathrm{p}}-S_{\mathrm{n}}\right), K$ is the total thermal conductance ( $\left.K=K_{\mathrm{p}}+K_{\mathrm{n}}\right), R$ is the series resistance $(R=$

(I) These parameters are related to material properties and dimension.
$R_{\mathrm{p}}+R_{\mathrm{n}}$ ), $\Delta T$ is the absolute temperature different between hot and cold side, and $I$ is a current. The first term is Peltier cooling, using the Thomson relation (Equation (2.4)) to connect Peltier coefficient and Seebeck coefficient. The second term is the thermal 
conduction. The last term comes from Joule heating. Increasing current will increase the Peltier cooling, however, the Joule heating will dominate since it depends on $I^{2}$ giving the COP a negative value. By differentiating the net heat with respect to current, we can find the maximum current as

$$
I_{\max }=\frac{S_{\mathrm{np}} T_{\mathrm{C}}}{R}
$$

This gives the maximum net heat

$$
Q_{\max }=\frac{\left(S_{\mathrm{np}} T_{\mathrm{C}}\right)^{2}}{R}-K \Delta T-\frac{1}{2}\left(\frac{S_{\mathrm{np}} T_{\mathrm{C}}}{R}\right)^{2}
$$

Next, the electrical power consumption in thermoelectric devices is given by

$$
W=S_{\mathrm{np}} I \Delta T+I^{2} R
$$

where the first term is from thermoelectric effect producing the voltage and the second is electrical power for external applied voltage. The COP for thermoelectric refrigerator for maximum heat output can be given by the ratio between the maximum net heat and electrical power consumption which leads to

$$
\mathrm{COP}=\frac{\frac{1}{2} Z T_{\mathrm{C}}^{2}-\Delta T}{Z T_{\mathrm{H}} T_{\mathrm{C}}}
$$

where $Z$ is the thermoelectric figure of merit of thermoelectric devices defined as

$$
Z=\frac{S_{\mathrm{np}}^{2}}{K R}
$$

Usually, one uses the dimensionless thermoelectric figure of merit $Z T_{m}$ (see below). Note, here we assume there are no heat resistance and electrical resistance between interconnect metals meaning that heat and electrical current from heat sink and source can flow from metal contact to thermoelectric material perfectly. ${ }^{22,33}$ For the maximum COP, the current that satisfies this condition is defined by

$$
I_{\max }=\frac{S_{\mathrm{np}} \Delta T}{R \sqrt{1+Z T_{m}}-1},
$$


where $T_{m}$ is an averaged temperature between hot and cold side, $T_{m}=\frac{\left(T_{\mathrm{H}}+T_{\mathrm{C}}\right)}{2}$. By using this current, we can calculate the maximum COP as

$$
\mathrm{COP}=\left(\frac{T_{\mathrm{C}}}{T_{\mathrm{H}}-T_{\mathrm{C}}}\right)\left(\frac{\sqrt{1+Z T_{m}}-T_{\mathrm{H}} / T_{\mathrm{C}}}{\sqrt{1+Z T_{m}}+1}\right)=\gamma \operatorname{COP}_{\mathrm{C}}
$$

Thus, the maximum thermoelectric refrigerator efficiency is a product of the Carnot cooling efficiency, and $\gamma$ is the weight of performance. For example, let us consider the two limiting cases, First $Z T_{m} \ll 1$ gives

$$
\mathrm{COP} \approx\left[\frac{T_{\mathrm{C}}}{\left(T_{\mathrm{H}}-T_{\mathrm{C}}\right)}\right]\left[\left(\frac{Z T_{m}}{2}\right)\left(1-\frac{T_{\mathrm{H}}}{T_{\mathrm{C}}}\right)\right],
$$

the efficiency is lower that Carnot efficiency. Second if $R \rightarrow 0$ and $K \rightarrow 0$, the thermoelectric device would have only (close to) reversible process, that is their $Z T_{m} \rightarrow \infty$ and their efficiency is the Carnot efficiency $\left(\mathrm{COP}=\mathrm{COP}_{\mathrm{C}}\right)$.

\subsubsection{Thermoelectric generation and efficiency of generator}

The simplest thermoelectric device for generating was shown in Figure 2.3(a). The energy conversion efficiency is obtained like the heat engine (see Equation (2.6)). Hence, we choose load resistance $\left(R_{\mathrm{L}}\right)$ in an appropriate temperature range to give maximum efficiency. This is shown by Ioffe, ${ }^{35}$ showed that this occurs when $M$ is the ratio of $R_{L} / R$, so it is defined by

$$
M=\frac{R_{\mathrm{L}}}{R}=\sqrt{1+Z T_{m}},
$$

where $Z T_{m}$ is the thermoelectric figure of merit, and $T_{m}$ is average temperature. The maximum efficiency is

$$
\eta=\left(\frac{T_{\mathrm{H}}-T_{\mathrm{C}}}{T_{\mathrm{H}}}\right)\left(\frac{\sqrt{1+Z T_{m}}-1}{\sqrt{1+Z T_{m}}+T_{\mathrm{H}} / T_{\mathrm{C}}}\right)=\varepsilon \eta_{\mathrm{C}}
$$

Therefore, we can see that the maximum thermoelectric generator efficiency is the Carnot efficiency, and the actual efficiency is scaled by the factor of efficiency $\varepsilon$, depending on the temperature of heat source and sink and thermoelectric figure of merit $\left(Z T_{m}\right)$. This leads to the same conclusion as for heat pumps that we need maximum $Z T_{m}$ in order to get maximum efficiency. 


\subsubsection{Thermoelectric Figure of Merit, geometrical considerations}

Typically, the figure of merit is represented as a dimensionless number by multiplying it with average temperature between heat source and sink, therefore thermoelectric figure of merit is written as $Z T_{m} . Z T_{m}$ is related to the properties of materials such as Seebeck coefficient $(S)$, thermal conductance $(K)$, and electrical resistance $(R)$. As we mentioned, to achieve $Z T_{m} \gg 1$, the product of $R K$ need to be minimized, i.e., the ratio of length and cross section of both sides needs to satisfy the condition ${ }^{22}$

$$
\frac{L_{\mathrm{n}} A_{\mathrm{p}}}{L_{\mathrm{p}} A_{\mathrm{n}}}=\left(\frac{\rho_{\mathrm{p}} \kappa_{\mathrm{n}}}{\rho_{\mathrm{n}} \kappa_{\mathrm{p}}}\right)^{1 / 2}
$$

According to this relation, it gives the figure of merit of a pair of thermoelectric materials as

$$
Z=\frac{\left(S_{\mathrm{p}}-S_{\mathrm{n}}\right)^{2}}{\left[\left(\rho_{\mathrm{p}} \kappa_{\mathrm{p}}\right)^{1 / 2}+\left(\rho_{\mathrm{p}} \kappa_{\mathrm{p}}\right)^{1 / 2}\right]}
$$

Since the thermoelectric figure of merit is written in this form, it is easy to interpret how good the materials are as thermoelectrics because there is no relation with the dimension.

\subsubsection{Basic consideration of improving thermoelectric figure of merit}

Let us now consider the dimensionless figure of merit of a single thermoelectric material, usually represented as

$$
Z T_{m}=\frac{S^{2} T_{m}}{\rho \kappa_{\mathrm{tot}}}
$$

where $\rho$ is the electrical resistivity, $S$ is the Seebeck coefficient, $\kappa_{\text {tot }}$ is the total thermal conductivity, and $T_{m}=\frac{\left(T_{\mathrm{H}}+T_{\mathrm{C}}\right)}{2}, T_{\mathrm{H}}$ and $T_{\mathrm{C}}$ are the absolute temperature at hot and cold end. In principle the dimensionless thermoelectric figure of merit is defined with average temperature between hot and cold end. But in practical the reported dimensionless thermoelectric figure of merit uses the ambient temperature instead due to the different of temperature between hot and cold end is quite small in order to avoid 
the non-linear effect on the Seebeck coefficient measurement (see Section (6.3.3) in Chapter 6). Thus the result after the average the temperature between hot and cold end is not different from ambient temperature, the dimensionless thermoelectric figure of merit can be rewritten into $Z T$ where is $T$ is the absolute temperature at the point Seebeck coefficient, electrical conductivity, and total thermal conductivity are measured.

If we can maximize the thermoelectric figure of merit of a material, it will be reflected on maximizing efficiency of a device. The individual $Z T$ value tells us that efficient thermoelectric of materials require high Seebeck coefficient, electrical conductivity, and low thermal conductivity. Although we know these basic requirements, the interrelationship of those parameters that determine the $Z T$ is an issue that impedes any further improvement of $Z T$. In this section, we will discuss about of improving $Z T$. From semiconductor physics and transport theory, the parameters in $Z T$ can be expressed in a simple model (parabolic band, energy-independent scattering approximation). ${ }^{4}$ The Seebeck coefficient $S$ is given by

$$
S=\frac{8 \pi^{2} k_{\mathrm{B}}^{2}}{3 e h^{2}} m^{*} T\left(\frac{\pi}{3 n}\right)^{2 / 3},
$$

and the electrical resistivity, $\rho$ can be expressed ${ }^{31}$ as

$$
\rho=\frac{1}{\sigma}= \pm \frac{1}{e n \mu}
$$

where $k_{\mathrm{B}}$ is the Boltzmann's constant, $e$ is the electron charge, $h$ is Planck's constant, $T$ is the absolute temperature, $m^{*}$ is the effective mass of the charge carrier, $\sigma$ is the electrical conductivity ,$n$ is the charge carrier concentration, and $\mu$ is carrier mobility, the plus and minus sign denotes the type of charge carrier is holes or electron, respectively. The total thermal conductivity, $\kappa_{\text {tot }}$ is

$$
\kappa_{\mathrm{tot}}=\kappa_{e}+\kappa_{l}
$$

It is a sum of carrier and lattice thermal conductivity since both electrons and lattice vibrations (phonon) can carry heat. ${ }^{4}$

For electronic thermal conductivity $4,36,37$

$$
\kappa_{e}=\sigma L T=\frac{L T}{\rho}
$$


where $\sigma$ is the electrical conductivity, $\rho$ is the electrical resistivity, $L$ is Lorentz factor, and $T$ is the absolute temperature. From kinetic theory we can derive the lattice thermal conductivity ${ }^{36,37}$ as

$$
\kappa_{l}=\frac{1}{3} C_{V}\left\langle\mathrm{v}_{g}\right\rangle l_{\mathrm{mfp}},
$$

where $C_{V}$ is the volume specific heat, $\left\langle\mathrm{v}_{g}\right\rangle$ is an average phonon velocity, and $l_{\mathrm{mfp}}$ is an average phonon mean free path. Therefore, when the carrier concentration is raised, the total thermal conductivity also increases. Here, we see that for example reducing the carrier concentration and increasing the effective mass of the material increases the Seebeck coefficient.

However, it directly affects the electrical conductivity because once you either decrease the carrier concentration or increase the carrier effective mass, the electrical conductivity is decreasing. Furthermore an increasing of carrier concentration is not a choice to improve $Z T$, because it will decrease the Seebeck coefficient and increase total thermal conductivity of the materials. Therefore, semiconductors have high potential to be efficient thermoelectric materials compare to metals or insulator since they allows us to optimize the carrier concentration. However, most semiconductors are covalently bonded yielding high total thermal conductivity. ${ }^{22}$ In next Chapter, we will try to breakdown these interrelationship in order to improve the $Z T$ of the materials. 



\title{
Chapter 3
}

\section{Thermoelectric figure of merit}

\begin{abstract}
"A person by study must try to disengage the subject from useless matter, and to seize on points capable of improvement. When subjects are viewed through the mists of prejudice, useful truths may escape."
\end{abstract}

Joseph MacSweeny in An Essay on Aerial Navigation, with Some

Observations on Ships (1844)

As pointed out at the end of the last chapter, it is not trivial to improve the thermoelectric figure of merit due to the interrelationship between thermoelectric parameters. We need to break down these thermoelectric parameters into more fundamental parameters which allow us individually optimize each parameters. In this chapter, we will go through the fundamental of each thermoelectric parameter i.e., Seebeck coefficient, electrical resistivity (conductivity), electron and lattice thermal conductivity. Then we will review the current strategies that are used to improve material thermoelectric figure of merit.

\subsection{Lattice dynamics}

The first thermoelectric parameter that we are going to discuss, is lattice thermal conductivity. The idea of heat is carried through the solid as the atomic lattice vibrational waves was proposed by Born and von Kármán ${ }^{38}$ as the dynamics theory of the lattice. They replaced the problem of the real crystal with many atoms that connected together by chemical bonds from electrons interactions with a classical system consists of harmonic oscillating point masses (ions)that obey translational periodicity connecting by massless electron springs. This simplified system is called harmonic lattice. This simplification allows them to approximate the complicated interatomic potentials with a simple Taylor expansion as a function of the atomic displacements $\mathbf{u}$ around lattice 
equilibrium positions. ${ }^{39,40}$ Thus the potential energy per unit cell of the crystal can be expanded as

$$
\begin{aligned}
U(\{\mathbf{u}\})= & U_{0}+\sum_{i} \sum_{\mu} \Phi_{i}^{\mu} u_{i}^{\mu}+ \\
& \frac{1}{2 !} \sum_{i j} \sum_{\mu \nu} \Phi_{i j}^{\mu \nu} u_{i}^{\mu} u_{j}^{\nu}+ \\
& \frac{1}{3 !} \sum_{i j k} \sum_{\mu \nu \xi} \Phi_{i j k}^{\mu \nu \xi} u_{i}^{\mu} u_{j}^{\nu} u_{k}^{\xi}+\ldots
\end{aligned}
$$

where $i j k$ are indices to atoms, $\mu \nu \xi$ Cartesian indices and $\{\mathbf{u}\}$ is a set of displacements for a crystal lattice with a basis, the basis vector is defined by $\tau_{i}$ and $\mathbf{R}_{i}$ is a lattice vector, thus the position of the atom is given by

$$
\mathbf{r}_{i}=\mathbf{R}_{i}+\tau_{i}+\mathbf{u}_{i}
$$

This notation is defined for convenient use since $\{\mathbf{u}\}$ is only dynamical parameter in this case. And the coefficients of the Taylor expansion are

$$
\begin{gathered}
\Phi_{i}^{\mu}=\left.\frac{\partial U}{\partial u_{i}^{\mu}}\right|_{u=0}=0 \\
\Phi_{i j}^{\mu \nu}=\left.\frac{\partial^{2} U}{\partial u_{i}^{\mu} \partial u_{j}^{\nu}}\right|_{u=0} \\
\Phi_{i j k}^{\mu \nu \xi}=\left.\frac{\partial^{3} U}{\partial u_{i}^{\mu} \partial u_{j}^{\nu} \partial u_{k}^{\xi}}\right|_{u=0} .
\end{gathered}
$$

Here $\Phi$ is defined as a force constant matrices and the subscript $u=0$ means expanding around lattice equilibrium positions. Let consider the polynomial terms in Equation (3.1), the constant first term, $U_{0}$ is related to equilibrium potential energy where all atoms sit on the lattice points. The first-order term is vanished because of the crystal should not have a net force when all atoms are placed at equilibrium position. Thus, there are only quadratic, cubic and higher order terms left. According to the assumption, we consider the system with small oscillation amplitude (displacement), therefore we can neglect all the all the higher order terms due to small contributions and keep only retain only the quadratic term. Thus our harmonic interatomic potential will look like

$$
U(\{\mathbf{u}\})=U_{0}+\frac{1}{2 !} \sum_{i j} \sum_{\mu \nu} \Phi_{i j}^{\mu \nu} u_{i}^{\mu} u_{j}^{\nu}
$$


This formula is similar to the harmonic oscillator potential in the classical mechanics. Thus the approximation of interatomic potential by Born and von-Kármán can be called harmonic approximation. ${ }^{36,37,39-42}$ Then we can use this approximated interatomic potential to determine the dynamic of the lattice. To reduce number of coupled equations of motion that we need to solve, we define new notation for denoting the atoms, the star vector $\mathbf{R}_{l}^{\alpha \beta}$. These star vectors are defined for each atom $i$ at position $\tau_{\alpha}$ in the unit cell we can define vectors that point to every atom $j$ at position $\tau_{\beta}$. This set of $l$ vectors we denote $\left\{\mathbf{R}_{l}^{\alpha \beta}\right\}$ and only depends on the indices to the positions in the unit cell, $\alpha \beta$, and not on the global atomic indices $i j$. From these star vector, we can write the Hamiltonian of the harmonic lattice,

$$
H=\sum_{i \alpha}\left(\frac{\mathbf{p}_{i \alpha}^{2}}{2 M_{i \alpha}}+\sum_{\beta} \sum_{l} \mathbf{u}_{i \alpha} \overline{\overline{\mathbf{\Phi}}}_{\alpha \beta}\left(\mathbf{R}_{l}^{\alpha \beta}\right) \mathbf{u}_{l \beta}\right) .
$$

where $M_{\alpha}$ is the mass of atom $\alpha, \Phi_{\alpha \beta}\left(\mathbf{R}_{l}^{\alpha \beta}\right)$ is a $3 \times 3$ interatomic force constant sub-matrix as a function of the star vector $\mathbf{R}_{l}^{\alpha \beta}$ for each atom pair at position in unit cell, $\alpha \beta$. And corresponding equations of motion of this harmonic lattice can be written as

$$
M_{\alpha} \ddot{\mathbf{u}}_{\alpha}=-\sum_{\beta} \sum_{l} \Phi_{\alpha \beta}\left(\mathbf{R}_{l}^{\alpha \beta}\right) \mathbf{u}_{l \beta}
$$

Since there are $N_{\text {cell }}$ number of unit cells and each unit cell has $r$ basis atoms, therefore we have $3 \times r N_{\text {cell }}$ Newton's equations of motion that need to be solved. Since this harmonic lattice is analog to the harmonic oscillator problem. The solutions of these equation of motion are in the form of plane wave which can be written as

$$
u_{\alpha}^{\mu}=\sum_{s, \mathbf{q}} \sqrt{\frac{\hbar}{2 N M_{\alpha} \omega_{s}(\mathbf{q})}} \boldsymbol{\epsilon}_{s}^{\alpha \mu}(\mathbf{q}) e^{i \mathbf{q} \cdot \tau_{\alpha}}\left(a_{\mathbf{q} s}+a_{-\mathbf{q} s}^{\dagger}\right)
$$

and its corresponding momentum

$$
p_{\alpha}^{\mu}=\sum_{s, \mathbf{q}} \frac{1}{i} \sqrt{\frac{\hbar M_{\alpha} \omega_{s}(\mathbf{q})}{2 N}} \boldsymbol{\epsilon}_{s}^{\alpha \mu}(\mathbf{q}) e^{i \mathbf{q} \cdot \tau_{\alpha}}\left(a_{\mathbf{q} s}-a_{-\mathbf{q} s}^{\dagger}\right)
$$

where $N$ is the total number of atoms in the system $N=r N_{\text {cell }}, \mathbf{q}$ is a wave vector in the first Brillouin zone which is corresponded 
to the periodic boundary condition proposed by Born and von Kármán, $\omega_{s}$ is a vibrational frequency and $\epsilon_{s}$ is the polarization vector as a function of $\mathbf{q}$, where $s$ is a branch index. To determine $\omega_{s}(\mathbf{q})$ and $\boldsymbol{\epsilon}_{s}(\mathbf{q})$ Next let substitute $u$ from Equation (3.9) into Equation (3.8), we can now determine the $\omega_{s}(\mathbf{q})$ and $\boldsymbol{\epsilon}_{\boldsymbol{s}}(\mathbf{q})$ from the eigenvalue problem,

$$
\omega_{s}^{2}(\mathbf{q}) \boldsymbol{\epsilon}_{s}(\mathbf{q})=\overline{\overline{\mathbf{\Phi}}}(\mathbf{q}) \boldsymbol{\epsilon}_{s}(\mathbf{q})
$$

Here the $\omega_{s}(\mathbf{q})$ and $\boldsymbol{\epsilon}_{\boldsymbol{s}}(\mathbf{q})$ can be determined by diagonalizing the "dynamical matrix" $\overline{\overline{\mathbf{\Phi}}}(\mathbf{q})$. This matrix has the dimension $3 r \times 3 r$, $r$ is the number of basis atom in unit cell and constructs from $3 \times 3$ sub-matrices $\overline{\overline{\boldsymbol{\Phi}}}_{\alpha \beta}(\mathbf{q})$

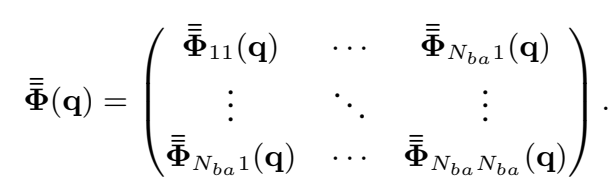

Each of the sub-matrix $\overline{\overline{\mathbf{\Phi}}}_{\alpha \beta}(\mathbf{q})$ is known as the reciprocal space dynamical matrix, is the Fourier transform of the constructed from the real space interatomic force constant matrices $\overline{\overline{\mathbf{\Phi}}}_{\alpha \beta}\left(\mathbf{R}_{l}\right)$ :

$$
\overline{\overline{\mathbf{\Phi}}}_{\alpha \beta}(\mathbf{q})=\sum_{l} \frac{e^{i \mathbf{q} \cdot \mathbf{R}_{l}}}{\sqrt{M_{\alpha} M_{\beta}}} \overline{\overline{\mathbf{\Phi}}}_{\alpha \beta}\left(\mathbf{R}_{l}\right),
$$

From the Equation (3.11),we obtain vibrational frequency (eigenvalue) $\omega_{s}(\mathbf{q})$ and polarization vector (eigenvector) $\boldsymbol{\epsilon}_{s}(\mathbf{q})$ as a multivalued function of $\mathbf{q}$. Since the $3 r \times 3 r$ dynamical matrix gives $3 r$ solutions for each wave vector $\mathbf{q}$ meaning that there are $3 r$ normal modes for each $\mathbf{q}$ which are identified by a branch index $s$. In other word there are $3 r$ of $\omega_{s}(1 \leq s \leq 3 r)$ for each $\mathbf{q}$ where $r$ is the number of basis atoms in unit cell. ${ }^{36}$

These $\omega(\mathbf{q})$ are then formed a dispersion relation, which describes the relation between vibrational frequency $\omega$ and the wavevector q. Due to symmetry of crystal, some of these normal modes can degenerate for a given $\omega$. Since there are $3 r$ normal modes for each $q$ value which are indexed by the branch index $s$. These normal modes can be categorized into 2 possible type depending on their behavior at the long wavelength limit $(\mathbf{q} \rightarrow 0)$. 
First type is acoustic modes, ${ }^{36,42}$ the vibrational frequency $\omega$ at small q shows linearly dependent before goes down to zero as $\mathbf{q} \rightarrow 0$. Since its dispersion relation behavior is similar to sound wave, thus this type of normal mode is called acoustic mode. The displacement of lattice neighboring atoms in acoustic mode will change in the same direction. Second type is optical modes, ${ }^{36,42}$ the $\omega$ does not drop to zero as $\mathbf{q} \rightarrow 0$ and this type of normal modes are strongly interact with optical wave in the ionic crystals (the ionic bond will form a strong electrical dipole). So we name this type of normal modes as optical modes. The displacement of neighboring atoms will displace in the opposite direction.

Here, three of these $3 r$ branches in dispersion belongs to acoustic mode (the number 3 comes from polarization directions, 2 longitudinal and 1 transverse polarization) and $3(r-1)$ branches are optical modes. ${ }^{36,42}$ For example, in the case of a crystal with 2 basis atoms per unit cell (a typical binary compound), there will be 3 branches are acoustic and 3 branches optical in a total of 6 branches. Since our system has $N_{\text {cell }}$ units cells, there should be $N_{\text {cell }}$ allowed of q according to Born and von Kármán and restriction to the first Brillouin zone. Thus we should have $N_{\text {cell }}$ of $\omega_{s}$ for each branch. Thus the total number of normal mode in our system equal to $3 r N_{\text {cell }}$.

According to the quantization language, we can transform harmonic Hamiltonian (Equation (3.7)) into a quantum mechanic operator which can be used to determine the energy of these normal modes. Thus the the coefficient $a$ and $a^{\dagger}$ in the position (Equation (3.9) and momentum (Equation (3.10)) have to transform into quantum mechanic operator called creation $\hat{a}^{\dagger}$ and annihilation $\hat{a}$ operator in harmonic oscillator. And these operators have commutator relations as following

$$
\begin{aligned}
& {\left[\hat{a}_{s \mathbf{q}}, \hat{a}_{s^{\prime} \mathbf{q}^{\prime}}^{\dagger}\right]=\delta_{\mathbf{q q}^{\prime}} \delta_{s s^{\prime}}} \\
& {\left[\hat{a}_{s \mathbf{q}}, \hat{a}_{s^{\prime} \mathbf{q}^{\prime}}\right]=\left[\hat{a}_{s \mathbf{q}}^{\dagger}, \hat{a}_{s^{\prime} \mathbf{q}^{\prime}}^{\dagger}\right]=0,}
\end{aligned}
$$

where $\delta_{s s^{\prime}}$ and $\delta_{\mathbf{q q}^{\prime}}$ are Kronecker delta. According to these commutation relations, they suggest that $\hat{a}_{s \mathbf{q}}$ and $\hat{a}_{s \mathbf{q}}^{\dagger}$ are used to create quasi-particle that satisfy Bose-Einstein statistic which is called "phonon". The name phonon is due to we are considering the atomic displacement with a certain set of vibrational frequencies (normal modes) that similar to the sound wave. From these 
creation and annihilation operator, we can write harmonic Hamiltonian operator as

$$
\hat{H}=\sum_{s, \mathbf{q}} \hbar \omega_{s}(\mathbf{q})\left(\hat{a}_{s \mathbf{q}}^{\dagger} \hat{a}_{s \mathbf{q}}+\frac{1}{2}\right)
$$

This harmonic Hamiltonian operator consists of $3 r N_{\text {cell }}$ independent oscillator Hamiltonians. For each of a single oscillator Hamiltonian with normal mode $\omega_{s}(\mathbf{q})$ can be written as

$$
\hbar \omega_{s}(\mathbf{q})\left(\hat{a}_{s \mathbf{q}}^{\dagger} \hat{a}_{s \mathbf{q}}+\frac{1}{2}\right)
$$

This normal mode of wave vector $\mathbf{q}$ of branch $s$ will contribute energy equal to

$$
E_{\mathbf{q} s}=\left(n_{s \mathbf{q}}+\frac{1}{2}\right) \hbar \omega_{s}(\mathbf{q}),
$$

where $n_{s \mathbf{q}}$ is the excitation states of normal mode of wave vector $\mathbf{q}$ of branch $s$ which is restricted to an integer values $0,1,2, \ldots$ In the second quantization language, we refer this excitation states as a state that contain $n_{s \mathbf{q}}$ number of phonons. To create and annihilate this phonon on this particular state that is identified by wave vector $\mathbf{q}$ of branch $s$, we then write

$$
\begin{aligned}
& \hat{a}_{s \mathbf{q}}^{\dagger}\left|n_{1 \mathbf{q}^{\prime}}, \ldots, n_{s \mathbf{q}}, \ldots\right\rangle=\sqrt{n_{s \mathbf{q}}+1}\left|n_{1 \mathbf{q}^{\prime}}, \ldots, n_{s \mathbf{q}}+1, \ldots\right\rangle \\
& \hat{a}_{s \mathbf{q}}\left|n_{1 \mathbf{q}^{\prime}}, \ldots, n_{s \mathbf{q}}, \ldots\right\rangle=\sqrt{n_{s \mathbf{q}}}\left|n_{1 \mathbf{q}^{\prime}}, \ldots, n_{s \mathbf{q}}-1, \ldots\right\rangle,
\end{aligned}
$$

and we define number operator $\hat{N}_{s \mathbf{q}}=\hat{a}_{s \mathbf{q}}^{\dagger} \hat{a}_{s \mathbf{q}}$, which satisfy,

$$
\hat{N}_{s \mathbf{q}}\left|n_{1 \mathbf{q}^{\prime}}, \ldots, n_{s \mathbf{q}}, \ldots\right\rangle=n_{s \mathbf{q}}\left|n_{1 \mathbf{q}^{\prime}}, \ldots, n_{s \mathbf{q}}, \ldots\right\rangle .
$$

Now the states of the entire of harmonic crystal are represented by the excitation states $n_{s \mathbf{q}}$, one for each of the $3 r N_{\text {cell }}$ normal modes. Therefore, the total energy of this lattice vibrations is just sum of the energies of each individual normal modes

$$
E_{\mathrm{tot}}=\sum_{s, \mathbf{q}}\left(n_{s \mathbf{q}}+\frac{1}{2}\right) \hbar \omega_{s}(\mathbf{q}) .
$$

It is clearly see that the energy of in the harmonic lattice is quantized into $3 N$ quantum numbers $n_{\mathbf{q} s}$. From the total energy expression, there is a term $\frac{1}{2} \hbar \omega_{s}(\mathbf{q})$ appears. This term is called zero point energy meaning that the lattice vibrational energy at the $0 \mathrm{~K}$ temperature, i.e., the atoms in the crystal still vibrate even the temperature of crystal equal to $0 \mathrm{~K}$. Since these phonons 
are quantized particle from lattice vibrations. Thus there are two types of phonon i.e., acoustic phonon from acoustic branches and optical phonon from optical branches where the neighbor atoms move in the opposite direction during vibration. Since phonons are quasiparticles, thus they should satisfy the Bose-Einstein partition function, ${ }^{42,43}$

$$
\mathcal{Z}=\prod_{s, \mathbf{q}} \frac{e^{-\beta \frac{\hbar \omega_{s \mathbf{q}}}{2}}}{1-e^{-\beta \hbar \omega_{s \mathbf{q}}}}
$$

where $\beta=-\frac{1}{k_{\mathrm{B}} T}$. From this partition function, we can obtain for example the Helmholtz free energy

$$
\begin{aligned}
F & =-k_{\mathrm{B}} T \ln \mathcal{Z} \\
& =\sum_{s, \mathbf{q}} \frac{\hbar \omega_{s \mathbf{q}}}{2}+k_{\mathrm{B}} T \ln \left(1-\exp \left(-\frac{\hbar \omega_{s \mathbf{q}}}{k_{\mathrm{B}} T}\right)\right) .
\end{aligned}
$$

For the convenience, we change from the sum over wave vector and branches into an integral over vibrational frequency, thus our phonon Helmholtz free energy can be expressed ${ }^{43}$ as

$$
F=\int_{0}^{\infty} g(\omega) \frac{\hbar \omega}{2}+g(\omega) k_{\mathrm{B}} T \ln \left(1-\exp \left(-\frac{\hbar \omega}{k_{\mathrm{B}} T}\right)\right) d \omega .
$$

where $g(\omega)$ is the phonon density of states which is given by

$$
g(\omega)=\sum_{s} \int_{\mathrm{BZ}} \frac{\delta\left(\omega-\omega_{s}(\mathbf{q})\right)}{2 \pi^{3}} d \mathbf{q} .
$$

Apart from the Helmholtz free energy, we can use to determine entropy by using the Helmholtz free energy, $\left(S=-\frac{\partial F}{\partial T}\right)$ at constant number of particle and volume or constant volume specific heat $C_{V}$. Since, phonons obey Bose-Einstein statistic, the equilibrium number of phonons in the mode $\mathbf{q}, s$ is given by BoseEinstein distribution functions:

$$
n_{s}^{0}(\mathbf{q})=\frac{1}{\exp \left(\hbar \omega_{s \mathbf{q}} / k_{\mathrm{B}} T\right)-1},
$$

where $k_{\mathrm{B}}$ is Boltzmann constant and $T$ is absolute temperature, and the chemical potential is equal to zero due to the total number of phonons in thermal equilibrium is not restricted. This distribution function can be considered as Planck distribution function. The average total energy of phonons is,

$$
\langle U\rangle=\sum_{s, \mathbf{q}} \hbar \omega_{s}(\mathbf{q})\left[n_{s}^{0}(\mathbf{q})+\frac{1}{2}\right] .
$$


And the constant volume specific heat can be written as

$$
C_{\mathrm{v}}=\frac{\partial\langle U\rangle}{\partial T},
$$

substitute $\langle U\rangle$ into the $C_{v}$, we got

$$
\begin{aligned}
C_{\mathrm{v}} & =\sum_{s, \mathbf{q}} \hbar \omega_{s}(\mathbf{q}) \frac{\partial}{\partial T} \frac{1}{e^{-\beta \hbar \omega_{s}(\mathbf{q})}-1}=\sum_{s, \mathbf{q}} \hbar \omega_{s}(\mathbf{q}) \frac{\partial n_{s}^{0}(\mathbf{q})}{\partial T} \\
& =C_{s \mathbf{q}}
\end{aligned}
$$

where $C_{s} \mathbf{q}$ is the mode specific heat capacity at wave function $\mathbf{q}$ and branch $s$. The concept of quantize vibrational lattice waves change our point of view in the heat conduction process i.e., we can imagine as heat generate particle name phonon that carry the thermal energy and propagate through the crystal instate of lattice vibrational wave. This consideration allows us explain most of thermal properties of the solid. Unfortunately, not all of thermal properties can be determined by harmonic approximation. There are several limitations in the harmonic approximation including thermal expansion is absent, thermal conductivity is infinite in the insulator where lattice thermal conductivity dominates, elastic constant is independent of pressure and temperature, etc.

There are two main reasons for the failure of harmonic approximation which are (i) harmonic approximation assume the crystal volume does not increase with temperature, (ii) missing higher order (anharmonic) term in the approximated interatomic potential. ${ }^{36,37,42}$ By missing higher order term leads to there are no phonon-phonon interactions meaning that phonons have infinite lifetime, thus they can conduct thermal energy (heat) forever similar to a ideal gas conduct heat in the infinite length cylinder. The problem about anharmonicity and how to obtain an accurate description of interatomic potential and force constant will be discuss in later Chapter 4.

\subsection{Lattice thermal conductivity}

By describing the lattice vibrations as quasi-particles, "phonons", allows us to use Boltzmann transport equation to describe the heat conduction in the solid. ${ }^{42}$ The phonon Boltzmann transport equation is obtained by assuming energy conservation in phonon 
scattering and using two approximations to simplify the Boltzmann transport equation, i.e., the linearized collision term and single mode relaxation time approximation ${ }^{42-44}$ which can be written as

$$
\mathbf{v}_{s \mathbf{q}} \nabla T \frac{\partial n_{s \mathbf{q}}^{0}}{\partial T}=-\frac{\left(n_{s \mathbf{q}}-n_{s \mathbf{q}}^{0}\right)}{\tau_{s \mathbf{q}}},
$$

where $n_{s q}$ is the nonequilibrium Bose-Einstein distribution function, $n_{s \mathbf{q}}^{0}$ is the equilibrium Bose distribution function (Equation (3.26)) at temperature $T, \mathbf{v}_{s q}$ is the phonon velocity, $\tau_{s q}$ is the total relaxation time, $s \mathbf{q}$ denotes the phonon mode of wave vector $\mathbf{q}$ and polarization (branch) $s$, and $T$ is the absolute temperature. Here, we assume that only one phonon mode with occupation number $n_{s \mathbf{q}}$ differs by a tiny amount from its equilibrium $n_{s \mathbf{q}}^{0}$. Considering the heat current density in direction $\alpha, J_{\alpha}$ :

$$
J_{\alpha}=\frac{1}{V} \sum_{s, \mathbf{q}} \hbar \omega_{s \mathbf{q}} \mathrm{V}_{s \mathbf{q} \alpha} n_{s \mathbf{q} \alpha}=-\kappa_{l} \Delta T
$$

where $V$ is the crystal volume, $\mathbf{v}_{s \mathbf{q} \alpha}=\frac{d \omega_{s \mathbf{q} \alpha}}{d \mathbf{q}_{s \alpha}}$ is phonon mode velocity, and $\alpha$ denotes a Cartesian direction. Substituting the $n_{s \mathbf{q}}$ from the single mode relaxation time approximation phonon Boltzmann transport equation (Equation (3.31)) and relate to lattice thermal conductivity $\kappa_{l},{ }^{42,44}$ we get

$$
\kappa_{\alpha \beta}=\frac{1}{V} \sum_{s, \mathbf{q}} C_{s \mathbf{q}} \mathrm{v}_{s \mathbf{q} \alpha} \mathrm{V}_{s \mathbf{q} \beta} \tau_{s \mathbf{q}},
$$

where $\kappa_{\alpha \beta}$ is the lattice thermal conductivity tensor for conduction in direction $\alpha \beta$, the $C_{s \mathbf{q}}$ is the general phonon mode specific heat from Equation (3.29), and $\alpha \beta$ denotes a Cartesian direction. Here, the term $\hbar \omega_{s \mathbf{q}} \mathrm{v}_{s \mathbf{q} \alpha} n_{s \mathbf{q} \alpha}^{0}$ is vanished due to the system should not contribute any conductivity at equilibrium. ${ }^{42-45}$ Here, we can change from the sum over wave vector into an integral over the vibrational frequency

$\kappa_{l}=\sum_{s} \frac{1}{3} \int C_{s}(\omega) \mathrm{v}_{s}^{2}(\omega) \tau_{s}(\omega) d \omega=\sum_{s} \frac{1}{3} \int C_{s}(\omega) \mathrm{v}_{s}(\omega) l_{s}(\omega) d \omega$,

where $l_{s}(\omega)$ is the frequency-dependent phonon mean free path $l_{s}(\omega)=\mathrm{v}_{s}(\omega) \tau_{s}(\omega), \tau_{s}(\omega)$ is the frequency-dependent phonon relaxation time, $\mathrm{v}_{s}(\omega)$ is frequency-dependent phonon velocity and $C_{s}(\omega)=\hbar \omega_{s} D(\omega)\left(\frac{\partial n_{s \omega}^{0}}{\partial T}\right)$ is the frequency-dependent specific heat with density of states $D(\omega)$ which is defined in Equation (3.25). Applying Debye approximation, ${ }^{36,42,43}$ the dispersion 
relation is linearized, $\omega(\mathbf{q})=\mathrm{v}_{g} \mathbf{q}$ and consider only contribution from acoustic branch meaning that we change integral over first Brillouin zone into integral over a sphere of radius $\omega_{D}$ chosen to contain precisely $3 N$ allowed normal mode where $N$ is the total number of atoms in crystal which equal to $r N_{c e l l}$, where $r$ is the number of basis atoms in the unit cell and $N_{\text {cell }}$ is the number of unit cells. Here we assume the crystal is thermally isotropic meaning that all the branches have similar phonon group velocity. The Debye frequency $\omega_{D}=\mathrm{v}_{g} q_{D}$. The generalize frequencydependent specific heat capacity can be written as

$$
C_{V}=9 N k_{\mathrm{B}}\left(\frac{k_{\mathrm{B}} T}{\hbar \omega_{D}}\right)^{3} \int_{0}^{x_{D}} \frac{e^{x}}{\left(e^{x}-1\right)^{2}} x^{4} d x
$$

where $k_{\mathrm{B}}$ is the Boltzmann constant, $\hbar=\frac{h}{2 \pi}$ is the reduce Planck constant, $N$ is the number of atoms in crystal which was defined above, $\omega$ is phonon frequency, $T$ is the absolute temperature, and $x=\frac{\hbar \omega}{k_{\mathrm{B}} T}$, and $x_{D} \equiv \frac{\hbar \omega_{D}}{k_{\mathrm{B}} T} \equiv \frac{\theta_{D}}{T}, \omega_{D}$ is Debye frequency and $\theta_{D}$ is Debye temperature. Substituting this Debye specific heat capacity into the lattice thermal conductivity, we obtain

$$
\kappa_{l}=\frac{k_{\mathrm{B}}}{2 \pi^{2} \mathrm{v}_{g}}\left(\frac{k_{\mathrm{B}} T^{3}}{\hbar}\right) \int_{0}^{x_{D}} \frac{\tau_{\text {tot }} x^{4} e^{x}}{\left(e^{x}-1\right)^{2}} d x .
$$

This lattice thermal conductivity is called Debye-Callaway lattice thermal conductivity. The $\mathrm{v}_{g}$ is a phonon group velocity $\mathrm{v}_{g}=$ $\frac{d \omega(\mathbf{q})}{d \mathbf{q}}$. This integral is depended on the total relaxation time $\left(\tau_{\text {tot }}\right)$, which can be expressed with Matthiessen's rule ${ }^{31,46}$ :

$$
\tau_{\text {tot }}^{-1}=\tau_{1}^{-1}+\tau_{2}^{-1}+\tau_{3}^{-1}+\ldots .
$$

Finally, we get the general expression for lattice thermal conductivity. ${ }^{47-49}$ Equations (3.34) and (3.36) suggest that reducing either one of these parameters, constant volume specific heat, phonon group velocity, or phonon relaxation time (introduce phonon scattering event) should result in decreasing of lattice thermal conductivity. Next, let consider the less thermoelectric parameters that we left in the beginning.

\subsection{Seebeck coefficient, electrical resistivity and electron thermal conductivity}

To maximize the thermoelectric figure of merit $Z T$, the Seebeck coefficient and the electrical conductivity need to be as large as 
possible, on the other hand the electron thermal conductivity is required to be as small as possible. It is hard to optimize each parameter individually. First we could neglect the electron thermal conductivity due to the fact that most of the thermoelectric materials are semiconductor. Thus we can assume that the lattice thermal conductivity in semiconductor is dominated over electron thermal conductivity due to "low" electrical conductivity compare to metal where the most contribution of thermal conductivity in metal comes from electrons. Furthermore, we had discussed the possibility about lowering lattice thermal conductivity in previous section. It is safely to say that the thermal conductivity in thermoelectric material is kind of under our control. This means that only Seebeck coefficient and electrical conductivity need to be maximized in order to get high $Z T$

In this section, we will consider the electrons (or holes) transport properties. Since electrons are charge particles that follow FermiDirac statistic. To examine, their transport properties in the solid, we again recall the linearized collision term and single mode relaxation time Boltzmann transport equation for electrons ${ }^{22,42,43}$ :

$$
\frac{f(E)-f_{0}(E)}{\tau_{\alpha}}=\mathrm{v}_{\alpha} \frac{\partial f^{0}(E)}{\partial E}\left(\frac{\partial E_{F}}{\partial \alpha}+\frac{\left(E-E_{F}\right)}{T} \frac{\partial T}{\partial \alpha}\right)
$$

Here, $f(E)$ is the non-equilibrium Fermi-Dirac distribution function, $f_{0}(E)$ is the equilibrium Fermi-Dirac distribution function at temperature $T, \mathrm{v}_{\alpha}$ is the velocity of the carriers in the $\alpha$ direction, $\tau_{\alpha}$ is the electrons relaxation time, $E_{F}$ is the Fermi energy (level), $T$ is the absolute temperature and $\alpha$ is the Cartesian direction. Here we used the same trick as in the case of phonon. The electrical current density $J_{\alpha}$ and electrical heat flux density $U_{\alpha}$ are

$$
\begin{gathered}
J_{\alpha}=\mp \int q \mathrm{v}_{\alpha} f(E) D(E) d E, \\
U_{\alpha}=\int \mathrm{v}_{\alpha}\left(E-E_{F}\right) f(E) D(E) d E,
\end{gathered}
$$

where $q$ is the electronic charge ( $-e$ for electron and $+e$ for hole), $D(E)$ is the electronic density of state (DOS). From these expression, we compare the term with Boltzmann transport equation above and eliminate the term relate to $f_{0}(E)$ due to no flow of any kind from the equilibrium distributions. Therefore we obtain 
a set of solutions from this Boltzmann transport equation:

$$
\begin{aligned}
\sigma_{\alpha} & =\frac{1}{\rho_{\alpha}}=\int \Sigma_{\alpha}(E)\left(-\frac{\partial f_{0}}{\partial E}\right) d E \\
S_{\alpha} & =\frac{k_{\mathrm{B}}}{q} \frac{\int \Sigma_{\alpha}(E)\left(\frac{E-E_{F}}{k_{\mathrm{B}} T}\right)\left(-\frac{\partial f_{0}}{\partial E}\right) d E}{\int \Sigma_{\alpha}(E)\left(-\frac{\partial f_{0}}{\partial E}\right) d E} \\
\kappa_{e \alpha} & =k_{\mathrm{B}}{ }^{2} T \int \Sigma_{\alpha}(E)\left(\frac{E-E_{F}}{k_{\mathrm{B}} T}\right)^{2}\left(-\frac{\partial f_{0}}{\partial E}\right) d E-T \sigma_{\alpha} S^{2} \\
& =\kappa_{0}-T \sigma_{\alpha} S^{2}
\end{aligned}
$$

where $\Sigma(E)$ is the transport distribution function,

$$
\Sigma(E)=\mathrm{v}_{\alpha}^{2}(E) \tau_{\text {tot } \alpha}(E) D(E) .
$$

Here $q$ is the carrier charge, $k_{\mathrm{B}}$ is the Boltzmann's constant, $T$ is the absolute temperature, $\sigma$ is the electrical conductivity, $S$ is the Seebeck coefficient, and $\kappa_{e}$ is the electron thermal conductivity, and $\kappa_{0}$ is the short circuit thermal conductivity, all parameters are expressed in case of applied field $\alpha$ direction where $\alpha$ is the Cartesian direction. Again all expression have the total electrons relaxation time $\tau_{\text {tot } \alpha}$ which follows Matthiessen's rule (Equation (3.37)). Finally, we have obtained the fundamental equations in thermoelectric applications. These equations suggest key parameters that are required to obtain high performance thermoelectric materials, i.e., the details of electronic band structure relate though density of state, charge carrier scattering mechanism, and the degree of degeneracy in materials. 


\subsection{Maximization of thermoelectric figure of merit}

Recall the thermoelectric figure of merit $Z T$ once again,

$$
Z T=\frac{S^{2} \sigma T}{\left(\kappa_{e}+\kappa_{l}\right)}=\frac{S^{2} T}{\rho\left(\kappa_{e}+\kappa_{l}\right)},
$$

where $S^{2} \sigma$ or $\frac{S^{2}}{\rho}$ is called power factor. According to our previous discussion, it is easily to realize that the lattice thermal conductivity is independent from the other thermoelectric parameters. Because of it depends the dynamics of the lattices, whereas the other parameters depend on the charge carriers (holes and electrons) conduction in the materials. This concept was first proposed by Slack as "Phonon-Glass Electron-Crystal (PGEC)". ${ }^{50}$ This idea is based on the fact that glass has the lowest lattice thermal conductivity, while crystals have good electrical properties. If we can combine these two features in the one material, the maximum $Z T$ should be obtained. Therefore, Slack suggested that the "best" thermoelectric material should have short phonon mean free path (short phonon relaxation time) at the same time the electron mean free path should really long (long carrier relaxation time). Base on this suggestion, there are two general ways how to improve the $Z T$ according to PGEC concept that is i) improve Seebeck coefficient $S$ and electrical conductivity $\sigma$ while keeping low lattice thermal conductivity and ii) reduce lattice thermal conductivity by maintaining power factor $S^{2} \sigma$.

\subsubsection{Minimizing lattice thermal conductivity}

The lattice thermal conductivity $\kappa_{l}$ can be minimized in three general ways (see Equation (3.34)), i) decreasing phonon group velocity $\mathrm{v}_{g}$ where $\mathrm{v}_{g}=\frac{d \omega(\mathbf{q})}{d \mathbf{q}}$, ii) lower constant volume specific heat, $C_{V}$, or ii) introducing phonon scattering mechanisms. Since $\mathrm{v}_{g}$ and $C_{V}$ are depended on the dispersion relation of materials phonon, $\omega(\mathbf{q})$. Therefore, if the material has loose chemical bond, large atomic mass ratio unit cell or both combined. Thus the complex materials or materials with many atoms per unit cell can be good candidate. Because of they should affect the $\omega(\mathbf{q})$ phonon dispersion relations result in low lattice thermal conductivity. The effect of bond softening effect can be seen in complex structure materials like Skutterudites or Clathrates. ${ }^{51-53}$ However, 
there is still a question on the lattice thermal conductivity mechanism i.e., the lattice thermal conductivity reduction occurs due to a resonant coupling between host and guest atom resulting in a deceasing phonon group velocity, or lattice disorder and point defects phonon scattering as suggested for skutterudites. ${ }^{54}$ Moreover the recently published results by Tadano et al. show that the low lattice thermal conductivity come from phonon-phonon scattering due to strong anharmonic effect induced by guest atoms. ${ }^{55}$ Note that the avoid crossing of the phonon modes is actually an effect of phonon-phonon scattering (see below for the discussion). Nevertheless, the clathrates and skutterudites have behavior follow the PGEC concept, i.e., low lattice thermal conductivity due to the coupling between host and guest atoms while Seebeck coefficient and electrical conductivity are relatively high due to charge carrier move along the host network results in these types of materials have high $Z T$ of about $1-1.25$ for skutterudites at 800 $900 \mathrm{~K}$ and $0.7-1$ for clathrates at $700-1000 \mathrm{~K} .^{54}$

Another type of material that follow PGEC concept is the natural inherently layered structures like layered oxide phase $\left(\mathrm{Na}_{x} \mathrm{CoO}_{2}\right.$, $\mathrm{Ca}_{3} \mathrm{Co}_{4} \mathrm{O}_{9}$, BiCuSeO oxyselenides, or $\mathrm{TiS}_{2}$ family). ${ }^{56-58}$ Alternating layers in these material structure can act as phonon scattering layers while providing another layer for charge carriers to pass through the materials results in high power factor $S^{2} \sigma$ while keeping low total thermal conductivity.

Apart from bond softening effect or inherit phonon scattering due to layer structure, the lattice thermal conductivity can be tailored by introducing phonon scattering mechanisms. This method can be combined with low lattice thermal conductivity materials which should result in a further decrease in lattice thermal conductivity. Next, we will discuss current interest phonon scattering mechanisms that are used nowadays.

\subsubsection{Phonon-phonon scattering}

Phonon-phonon scattering is an intrinsic property of materials as a result from the anharmonic interatomic potential. This scattering phenomenon will not occur, if the atomic bonding potential is a purely harmonic potential. ${ }^{36,37,42}$ Because the interatomic potential was truncated at second-order term, thus the phonon- 
phonon scattering description is missing. To include the phononphonon scattering description in the theory, the higher-order anharmonic term in the interatomic potential expansion need to be include. The third-order, forth-order, and so on will create corresponding three, four, and etc. phonons scattering, respectively. For the simplicity, we will discuss only three phonons scattering here. This three phonons can be explained as a lattice vibrational wave with wave vector $\mathbf{q}$ propagate in the anharmonic crystal. Due to the anharmonic forces some atoms move closer than their equilibrium position and some move further apart. This effect gives a local change of elasticity in the crystal because of some area lattices are over compressed and some are over expanded. Suppose there is another lattice vibrational waves with wave vector $\mathbf{q}^{\prime}$ tries to propagate in this anharmonic crystal; it will be reflected with a new wave vector $\mathbf{q}^{\prime \prime}$. Since the elasticity of the medium is changed due to a lattice vibrational waves $\mathbf{q}$. This situation similar to wave propagate into difference medium.

There are two types of three phonons scattering, Normal process (N-process) and Umklapp-process (U-process). ${ }^{36,41,42} \mathrm{~N}$-process occurs three phonons scatter with conserved total momentum in the unit cell i.e., $\mathbf{q}+\mathbf{q}^{\prime}=\mathbf{q}^{\prime \prime} .{ }^{36,37,41,42}$ On the other hand, $\mathrm{U}$ process needs to generate another phonon with additional momentum as a reciprocal lattice vector $\mathbf{G}$ to conserve the total phonon momentum in the unit cell i.e., $\mathbf{q}+\mathbf{q}^{\prime}=\mathbf{q}^{\prime \prime}+\mathbf{G} \cdot{ }^{36,37,41,42}$ Therefore, we can consider N-process as phonon redistributing process which yields indirect thermal resistivity and U-process will lead to direct thermal resistivity due to the scattered phonon is backscattered back into unit cell by phonon with momentum of reciprocal lattice vector meaning phonon is lost into another unit cell. The phonon relaxation time for Umklapp- and Normalprocess were suggested by Callaway ${ }^{47,49,59}$ as

$$
\tau_{U}(\omega)=\frac{1}{B_{U} \omega^{2} T e^{\theta_{D} / 3 T}} \quad \text { and } \quad \tau_{N}(\omega)=\frac{1}{B_{N} \omega^{2} T^{3}} .
$$

The constant $B_{U}$ and $B_{N}$ can be approximated as

$$
B_{U} \approx \frac{\hbar \gamma^{2}}{M \mathrm{v}_{g}^{2} \theta_{D}} \quad \text { and } \quad B_{N} \approx \frac{k_{\mathrm{B}}{ }^{3} \gamma^{2} V}{M \hbar^{2} \mathrm{v}_{g}^{5}}
$$

where $\theta_{D}$ is the Debye temperature $\theta_{D}=\frac{\hbar \omega_{D}}{k_{\mathrm{B}}}, M$ is the average mass of atoms in the crystal, $\mathrm{v}_{g}$ is the phonon group velocity, $\gamma$ is 
the Grüneisen parameter, $V$ is the crystal volume and $T$ is the absolute temperature. This expression taken from Morelli et al. Note that we still assume the thermally isotropic crystal. Otherwise, the $\theta_{D}, \mathrm{v}$, and $\gamma$ will be depended on longitudinal or transverse branches. Here we can consider that the U-process will typically dominate as the temperature increases, hence high temperature will generate many high momentum phonons with sufficiently to make them scatter outside the unit cell. This is the reason why total thermal conductivity decreases when the temperature increase in semiconductor and insulator.

This phonon-phonon scattering can be consider as an intrinsic property of materials. Since it depends on the degree of anharmonicity of the material which is related to the type of elements in the compound, strength and type of chemical bonds, and crystal structure. Thus we could engineer the material with desired anharmonicity by achieving the nesting of phonon dispersion relations via the charge carrier doping, chemical pressure, or tailoring the mass ratio to further reduce thermal conductivity.

\subsubsection{Phonon scattering by impurities or alloying atoms}

Anharmonicity is not only one effect that can change the local elasticity inside the crystal. When the point defects or alloying elements have incorporated in solid, they will create the distortion of the strain field (elasticity fluctuation) due to difference on chemical bonding and the mass fluctuation resulting in phonon scattering. Klemens first suggested the mathematical expression for all these effects. ${ }^{45,60,61}$

$$
\tau_{\text {imp }}=\frac{4 \pi \mathrm{v}_{g}^{3}}{V \Gamma \omega^{4}}
$$

Here Klemens applied the Debye approximation to derive this equation for $\tau_{\text {imp }}$. The general form of $\tau_{\text {imp }}$ can be written as

$$
\frac{1}{\tau_{\text {imp }}}=\frac{6 \Gamma \omega^{2} D(\omega)}{\pi V}
$$

where $V$ is the crystal volume, $\mathrm{v}_{g}$ is the phonon group velocity, $\omega$ is the vibrational frequency, $\Gamma$ is the scattering parameter, and $D(\omega)$ is the phonon density of states which equal to $\frac{3 \omega^{2}}{2 \pi^{2} \mathrm{v}_{g}^{3}}$. 
Klemens also suggested the scattering parameter $\Gamma$ for impurities phonon scattering relaxation time as

$$
\begin{aligned}
\Gamma & =\sum_{i} \Gamma_{i} \\
& =\sum_{i} x_{i}\left\{\left(\frac{\Delta M}{M}\right)^{2}+2\left[\left(\frac{\Delta K}{K}\right)-2 Q\left(\frac{\Delta R}{R}\right)\right]^{2}\right\},
\end{aligned}
$$

where $x_{i}$ is the frequency of the $i^{\text {th }}$ isotope or a fractional concentration of the $i^{\text {th }}$ species for alloy case, $M_{i}, K_{i}$ and $R_{i}$ are the atomic mass, bulk modulus, and bonding length of the matrix (or the concentration average for the alloy case) and $\Delta M$ and $\Delta K$ are the different of each quantity between matrix and impurities (including isotope) or alloying atoms. $\Delta R$ is the difference of local bonding length caused by a point defect. $Q$ is accounted for the accumulative influences on surrounding bonds. When the host atom is replaced by substitutional impurity or vacancy, the influences on the boning length is different. The substitutional impurity should contribute more than vacancy due to substitutional impurity does not fit well with lattice creating more distortion in the crystal, but vacancy is loosen the bonding. To accounting this fact, Klemens suggested $Q_{\text {imp }}=4.2$ for substitutional impurity and $Q_{\mathrm{vac}}=3.2$ for vacancy. ${ }^{60}$

Later Abeles ${ }^{62}$ improved this expression for alloying by replacing the bonding length term with $\delta d / R$ where $\delta a$ is the difference of lattice constant of matrix and alloy in their own lattice so the new $\Gamma$ for binary $\left(A_{1-x} B_{x}\right)$ or pseudo-binary $\left((A B)_{1-x}(A C)_{x}\right)$ is

$$
\Gamma=x(1-x)\left[\left(\frac{\Delta M}{M}\right)-\varepsilon\left(\frac{\Delta a}{a}\right)\right],
$$

where $\Delta M$ and $\Delta a$ are the difference in mass and lattice constants between two constituents and $M$ and $a$ are the molar mass and lattice constant of the alloy. The parameter $\epsilon$ is related to the Grüneisen parameter $\gamma$, the ratio of bulk modulus $\left(\frac{\Delta K}{K}\right)$ and the local bonding length $\left(\frac{\Delta R}{R}\right) G$ and Poisson ratio $P$ :

$$
\varepsilon=\frac{2}{9}\left[(G+6.4 \gamma) \frac{1+P}{1-P}\right]^{2} .
$$

From the scattering parameter $\Gamma$ suggests that we can achieve low lattice thermal conductivity by alloying with element that create the largest distortion weather in mass, elasticity, or bond 
distortion. This is the simplest approach to reduce lattice thermal conductivity for several decades until now. Due to the fact that the solid solution alloy can provide the carrier concentration optimization meaning that we can improve power fact $S^{2} \sigma$ and reduce $\kappa_{l}$ at the same time. However, the alloying can act as scattering center for the charge carrier which will reduce carrier mobility according to the carrier relaxation time for alloy scattering. Since the carrier alloy scattering relaxation time

$$
\tau_{\text {ca-al }}(\xi)=\frac{8 \hbar^{4}}{3 \sqrt{2} \pi \Omega x(1-x) U_{\text {ca-al }}^{2}\left(m_{b}^{*}\right)^{3 / 2}} \xi^{-1 / 2},
$$

where $\Omega$ is the volume per atom, $x$ is the molar fraction, $U_{\text {ca-al }}$ is the alloy scattering potential, $m_{b}^{*}$ is the density of state effective mass. This expression is for binary alloy nondegenerate parabolic semiconductor and $\xi$ is the reduce carrier energy, $\xi=\frac{E}{k_{\mathrm{B}} T}$. This carrier scattering comes from the discontinuity of crystal potential energy due to replacing the large amount of host atoms in the crystal with alloying atoms. This results in the fluctuation in the conduction (valence) band edge which scatters the carrier.

From the relaxation time equation, it suggest that the shortest relaxation time (low electrical conductivity see Equation (3.41)) occurs when $x(1-x)$ is maximum at $x=0.5$. Furthermore, the carrier alloy scattering rate depended on the carrier alloy scattering potential $U_{\text {ca-al }}=U_{\mathrm{A}}-U_{\mathrm{B}}$. Thus the choice of alloy element is important. We need the alloy elements have highest efficiency, i.e., the alloy atoms should effectively scatter only phonon and avoid scatter with carrier scattering. This suggest that the alloy atom should have heavy mass with large number of stable isotope (a lot mass fluctuation) and exhibit the lowest carrier alloy scattering potential $U_{\text {ca-al }}$. Unfortunately, the true factor that determining $U_{\text {ca-al }}$ is still on debated between whether it comes from difference in electron affinity of alloying and matrix elements or the different between energy band edge alloy cluster/atom and matrix. Thus we need to consider both facts for picking the suitable alloy elements.

If we consider the frequency dependent of phonon relaxation times that were considered so far. We should notice that there is no mechanism that can scatter mid and low frequency phonons leading to a large portion of heat can still be conducted by these mid and low frequency phonons. Because of the intrinsic phononphonon scattering cannot scatter all phonons in the frequency 
spectrum, only high frequency one is scattered due to $\tau_{\mathrm{U}} \propto \omega^{-2}$. And it can be improved by an alloys scattering since $\tau_{\text {imp }} \propto \omega^{-4}$. Due to the alloy scattering most of the high frequency phonon should be taken out. In order to scattering these mid to low frequency phonon in order to reduce lattice thermal conductivity furthermore, the grain boundary need to be introduced. Next we will discuss about grain boundary scattering of phonons.

\subsubsection{Phonon scattering by grain boundary scattering}

Grain boundaries can also affect the phonon conduction since they can limit the phonon mean free path. The relaxation time of grain boundary scattering is shown by

$$
\tau_{G}=\frac{L_{0}}{\mathrm{v}_{g}},
$$

where $\mathrm{v}_{g}$ is the phonon group velocity and $L_{0}$ is the effective grain size which is defined as $L_{0}=D$ for a crystal with cylindrical shape with cross-section of diameter $D$ and $L_{0}=1.12 d$ for a square cross-section of size $d .{ }^{63}$ This expression assume that the grain boundary is perfectly rough which is not the case in reality. Thus, we can modify an effective grain boundary length by including the specularity factor $p$,

$$
L=\frac{(1+p)}{(1-p)} L_{0}
$$

Here, the specularity factor is a limiting parameter, i.e., $p=1$ meaning the grain boundary scattering is purely specular scattering and the opposite case $p=0$ resulting in the grain boundary scattering is purely diffusive scattering (Casimir regime). ${ }^{63,64}$ Typically the grain size that required scattering phonon is in order of $10^{-6} \mathrm{~m}$ or less. Thus the polycrystalline material or nanocomposite ${ }^{\mathrm{I}}$ material that have grain size in the nanometer range should have low thermal conductivity due to effective scattering low frequency phonon. Because of this approach, it is the main reason of a huge improvement in material $Z T$ in the past decades. The low-dimension materials as thin film, quantum well/quantum dot structure, or nanowire can also benefit from the grain boundary phonon scattering mechanism. ${ }^{65-69}$ Moreover, the phonon scattering at interface is also possible which contributes to further decrease of lattice thermal conductivity. The alternating thin layer of two materials in superlattice structure can couple
(I) a material that contain two or more phases, segregated in the nanoscales. 
between alloying and size effects yielding the reduction of lattice thermal conductivity. ${ }^{70-72}$ However, grain boundary can also affect electrical conductivity due to carrier scattering at the boundary. This is because of the incoherent interface between grains resulting in carrier scattering by the discontinuity of the crystal potential. Thus if one could synthesis a nanostructure material consist of nanoinclusions that have coherent interface with the matrix. The carrier scattering interface should be minimized.

This idea was shown by Biswas et al. They synthesized a strained coherent interface nanoinclusions of SeTe in PbTe matrix due to phase segregation phenomena. In their study, they reported that this nanostructure can reduce lattice thermal conductivity while retaining electrical conductivity yielding an improvement of $Z T$ from 0.9 to 1.6 around $700 \mathrm{~K} .{ }^{73}$ In addition, this nanostructure is a nanocomposite. We would consider that the carrier should possible to scattering due to potential barrier between host matrix and nanoinclusions. However this is not the case, due to the energy band alignment between host matrix and nanoinclusions such a scattering mechanism is reduced.

\subsubsection{Nanostructure all-scale hierarchical architecturing}

This idea is based on the fact that different type of scattering mechanisms scatter phonon with different wavelength. If we can utilize all phonon scattering mechanisms together the lowest lattice thermal conductivity should be obtain. This idea was suggested by Zhao et al. suggest the method so called "all-scale hierarchical architecting". ${ }^{21}$ In order to achieve that goal different strategies are combined, introduction of dopant atoms on the lattice site by forming solid solution will enable short wavelength phonon scattering. Then forming a nanocomposite in order to scattering medium wavelength phonon via effectively be scatter by interface between nanoinclusions and matrix. The last portion of phonon that left in the crystal is long wavelength phonon. These long wavelength phonons can be scattered by grain boundary of the grain size in a range of $100 \mathrm{~nm}$ to $5 \mu \mathrm{m}$.

If all these steps are combined, the lattice thermal conductivity can significantly be reduced down to the minimum theoretical limit. Since, nanoinclusions has coherent interface with energy 
band alignment with the matrix; and the grain size of matrix is large enough so carriers are not suffer from collision. This type of architecture of nanostructure materials should substantial improve on the $Z T$. The result of this idea was shown by Biswas et al. ${ }^{74}$ They show that they can improve the $Z T$ in PbTe:SrTe with Na doping system about 1.1 to 2.2 with all-scale hierarchical architecting. The word "all-scale" means we are consider to scatter phonon from by atomic-, nano-, and meso-scales structures. ${ }^{21}$

Another idea that similar to hierarchal architecting was reported by Kim et. al. ${ }^{75}$ Instead of create participate inside the material they created the array of dislocation in the filled grain boundary. Since, the dislocation can also locally disturb an elasticity results in phonon scattering. ${ }^{76}$ This array of dislocations at the grain boundary can reduced the heat that carry from medium phonon frequency by $45 \%$. Since the grain boundary was filled with excessed Te. This excessed Te then forms a semi-coherent between interfaces of each grains. Therefore electron has pathway to move from grain to grain resulting electrical conductivity is improved compare to not fill grain boundary. According to these feature, they can improved the $Z T$ of $\left(\mathrm{Bi}_{0.5} \mathrm{Sb}_{1.5}\right)_{2} \mathrm{Te}_{3}$ from 1 to 2 at $320 \mathrm{~K}$.

Despite the fact that the lattice thermal conductivity reduction is the easiest way of improving $Z T$. After we reach the amorphous limit for lattice thermal conductivity. The thermal conductivity cannot be reduced any further. Accord to this fact, this lead us to incorporate between lattice thermal conductivity reduction and thermoelectric power improvement. Next we will discuss on the possibility of improving thermoelectric power factor while we keep lattice thermal conductivity low or the way that we can reduce lattice thermal conductivity without decreasing thermoelectric power factor. 


\subsubsection{Improving Seebeck coefficient and electrical conductivity}

Here we will discuss how we can improve the thermoelectric power factor $S^{2} \sigma$,

\subsubsection{The "best" electronic band structure for thermoelectrics}

In 1996 Mahan and Sofo made another analysis on finding the conditions for the best thermoelectrics as general as possible. ${ }^{77}$ They applied the Boltzmann transport equation to express three transport coefficients for thermoelectric which are depended on the transport distribution function, $\Sigma(E)$ and is defined by

$$
\Sigma(E)=\sum_{\mathbf{k}} \tau_{\text {tot }}(\mathbf{k}) \mathrm{v}_{x}^{2}(\mathbf{k}) \delta(E-E(\mathbf{k})) .
$$

For parabolic band structure, the transport distribution function can be written as

$$
\Sigma(E) \approx \tau_{\text {tot }}(E) \overline{\mathrm{v}}_{x}^{2}(E) D(E),
$$

which is similar to Equation (3.41) (3.42) and (3.43) where, $\mathrm{v}_{x}(\mathrm{E})$ is the group velocity of the carriers with energy $E$ in the direction of the applied field $x, \overline{\mathrm{v}}_{x}^{2}(E)$ is the average group velocity of the carriers, $\tau_{\text {tot }}$ is the total relaxation time of the carriers, and $D(E)$ is electronic density of states (DOS). According to their mathematical analysis, Mahan and Sofo concluded that the best thermoelectric materials should have a transport distribution function in a form of Dirac delta function. Thus they suggested that in the case of charge carriers energy independent relaxation time $(\tau(E)=C$, where $C$ is a constant), the shape of transport distribution function will only depend on the electronic DOS function. Therefore the material that has an electronic DOS exhibits very narrow and high peak at Fermi level, that material should result in the maximum $Z T .{ }^{77}$ Later Fan et al., generalized the Mahan and Sofo suggestion. ${ }^{78}$ Their conclusions agree with Mahan and Sofo suggestion. They also stressed that, transport distribution function needs to be as high and narrow as possible within the Fermi window $\left(k_{\mathrm{B}} \mathrm{T}\right)$ to maximize electrical conductivity and as asymmetric as possible with respect to the Fermi level to enhance the Seebeck coefficient. 
This approach of enhancing Seebeck coefficient by asymmetric electronic DOS can be related to the Mott equation. ${ }^{79-81}$ For degenerate semiconductor or metals, it can be written as

$$
\begin{aligned}
S & =-\left.\frac{\pi^{2} k_{\mathrm{B}}^{2} T}{3 e} \frac{d \ln \sigma(E)}{d E}\right|_{E=E_{F}} \\
& =-\left.\frac{\pi^{2}{k_{\mathrm{B}}}^{2} T}{3 e}\left(\frac{1}{D(E)} \frac{d D(E)}{d E}+\frac{1}{\mu} \frac{d \mu(E)}{d E}\right)\right|_{E=E_{F}},
\end{aligned}
$$

where $k_{\mathrm{B}}$ is Boltzmann's constant, $e$ is the electron charge, $T$ is the temperature, and $\mu$ is the carrier mobility. The Mott equation shows that the Seebeck coefficient can be increased when the electronic DOS and carrier mobility are strongly dependent on energy at Fermi level $\left(E_{F}\right)$. Therefore, if the material has very steep slope in the electronic DOS, this will result in a enhancement in Seebeck coefficient. This steep slope feature on the electronic DOS can be obtained from intrinsic properties of materials i.e., materials consist of localized orbital elements which may contribute a shape feature in electronic DOS around Fermi level. Or it can be engineered. There are two ways that have been suggested for engineering such a feature :

i) The distortion of the electronic density of states through low dimension materials. ${ }^{5,82}$ This effect appears when the dimension or structure of the materials is reduced from bulk material (3D) to quantum well (2D), quantum wire (1D), or quantum dot (OD) structures, the electronic band structure will be changed accordingly which generate localized states yielding the modification of the electronic density of states. For example it is shown in the $\mathrm{PbTe}$ nanowire.

ii) The distortion of the electronic density of states through stoichiometry tuning and doping. ${ }^{79,83}$ These point defects can form resonant or localize levels in energy band yielding asymmetry sharp peaks on electronic density of state. ${ }^{83}$ This approach has been demonstrated by Heremans et al., ${ }^{79,83}$ who obtained $Z T=$ 1.5 at $773 \mathrm{~K}$ in Tl-doped PbTe which is twice as large as p-type PbTe-based alloys. By doping PbTe with $2 \%$ of $\mathrm{Tl}$, the Seebeck coefficient increased due to Tl-induced peaks in electronic DOS around Fermi level. It is not only $\mathrm{Tl}$ that cause the resonant levels in $\mathrm{PbTe}, \mathrm{Ti}, \mathrm{Cr}$, and In can cause the resonant level in PbTe as well. ${ }^{83,84}$ This similar effect also observe in $\mathrm{Mg}_{2} \mathrm{Si}_{1-\mathrm{x}} \mathrm{Sn}_{\mathrm{x}}$ where 
$Z T$ can improve from 0.7 in the binary to 1.2 in the entire of temperature. ${ }^{83}$ However, this distortion of the electronic density of states may lead to undesired results. We will discuss in following section.

\subsubsection{The materials parameter, $\mathrm{B}$}

The $Z T$ can be rewritten in term of the reduce Fermi energy, $\eta=\frac{E_{F}}{k_{\mathrm{B}} T}$. For non-degenerate semiconductor in which MaxwellBoltzmann statistics can be used for the electrons and holes instead of Fermi-Dirac statistics, the new form of $Z T$ can be expressed as

$$
Z T=\frac{[\eta-(r+5 / 2)]^{2}}{(B \exp \eta)^{-1}+(r+5 / 2)} .
$$

The $Z T$ becomes a function of the reduce Fermi energy $\eta$, charge carrier scattering coefficient $r$, and the parameter includes properties of material which is called materials parameter $B$. This parameter was first considered by Chasmar and Stratton ${ }^{85}$ and is defined by

$$
B=\left(\frac{k_{\mathrm{B}}}{q}\right)^{2} \frac{\sigma_{0} T}{4 \kappa_{l}},
$$

where $\sigma_{0}$ is a quantity relates to the carrier mobility and the effective mass which can be described by

$$
\sigma_{0}=2 q \mu N_{\mathrm{v}}\left(\frac{2 \pi m_{d}^{*} k_{\mathrm{B}} T}{h^{2}}\right),
$$

where $h$ is Planck's constant, $k_{\mathrm{B}}$ is Boltzmann's constant, $q$ is an electron charge ( $-e$ for electron and $+e$ for hole), $\mu$ is a charge carrier mobility, $N_{\mathrm{v}}$ is the number of equivalent bands extrema (the maximum of valence band and the minimum of conduction band), i.e., valley degeneracy because of different spin-orbital states and valley ellipsoids, $m_{d}^{*}$ is the carrier density of states effective mass, $\tau_{\text {tot }}$ is the total relaxation time of carrier, $T$ is temperature, and $\kappa_{l}$ is the lattice thermal conductivity. The $Z T$ will increase as the material parameter is increased. If we neglect all the fundamental constants, the materials parameter is proportional to a weighted mobility, $\mu \cdot\left(m_{d}^{*} / m_{e}\right)^{3 / 2}$, where $m_{d}^{*}$ is the carrier density of states effective mass, and $m_{e}$ is the electron mass. ${ }^{22,50}$ Heavy carriers and high mobility are desired features for maximum $Z T$ materials. However, $\mu$ and $m_{d}^{*}$ are related to each other via $\mu=q \tau / m_{c}^{*},{ }^{31,86,87}$ where $q$ is carrier charge $(-e$ 
for electron and $+e$ for hole), $m_{c}^{*}$ is the conduction effective mass which is defined as

$$
m_{c}^{*}=3\left[\left(1 / m_{1}^{*}\right)+\left(1 / m_{2}^{*}\right)+\left(1 / m_{3}^{*}\right)\right]^{-1},
$$

and

$$
m_{d}^{*}=N_{\mathrm{v}}^{2 / 3} \cdot\left(m_{1}^{*} m_{2}^{*} m_{3}^{*}\right)^{1 / 3}=N_{\mathrm{v}}^{2 / 3} m_{b}^{*}
$$

where $N_{\mathrm{v}}$ is the number of equivalent carrier pockets (valley degeneracy), $m_{1}^{*}, m_{2}^{*}$, and $m_{3}^{*}$ are the inertial effective mass of carrier in three principle axis of a carrier pockets (single valley) which obtained from the effective mass tensor, and $m_{b}^{*}$ is the average band effective mass (single pocket density of states effective mass). ${ }^{31,86,87}$

Since the density of state effective mass and carrier mobility are connected with a inertial effective mass tensor that is defined as a second derivative of energy band, $E(\mathbf{k})$ with respect to wavevector, $\mathbf{k}$ (the curvature of energy band $\frac{1}{m_{i j}^{*}}=\hbar^{-2} \frac{\partial^{2} E(\mathbf{k})}{\partial k_{i} \partial k_{j}}$, where $i$ and $j$ equal to 1,2 , and 3 ). In case of the flat or broad energy band meaning a narrow electronic DOS, the carrier will have a heavy mass with low carrier mobility and vice versa. Generally, there is no universal requirement whether heavy effective mass or high mobility ${ }^{4}$ such as oxides or chalcogenides that carrier has a heavy effective mass with low mobility ${ }^{88}$ or SiGe that carrier has a light effective mass with high mobility. ${ }^{89}$ Now let me refer back to the previous section, if our doping creates a narrow feature in electronic DOS, i.e., flatten the energy band. It will indeed enhance Seebeck coefficient of material according to Mott formula. However, this enhancement of Seebeck coefficient will get compensated by electrical conductivity reduction due to a heavy inertial effective mass from a flat energy band. Thus the energy band distortion may not be a good way to improve the power factor. Nevertheless, there are some possible approaches that can decouple the relation between $\mu$ and $m_{d}^{*}$, so the material parameter can be optimized in order to achieve high $Z T$ materials.

The first approach was introduced by Hicks and Dresselhaus. ${ }^{90}$ They showed that multilayer or superlattice structures can be used to optimize the materials parameter by changing the layer thickness and choosing the optimum current direction. A narrow layer thickness will increase $B$ and choosing the best orientation of the layer structure in which either mobility or effective mass can be 
maximized. They showed that if $\mathrm{Bi}_{2} \mathrm{Te}_{3}$ superlattice is prepared in the $a-c$ plane and the carriers flow along $c$ axis which yield the highest mobility, the $Z T$ of 13 can be obtained, in the theory. If the superlattice of $\mathrm{Bi}_{2} \mathrm{Te}_{3}$ is prepared in conventional way, i.e., in $a$ - $b$ plane, a threefold increase in $Z T$ was predicted for $10 \AA$ layer thickness. ${ }^{90}$

The second approach is depended on the density of states effective mass which is defined in Equation (3.63). The equation shows that a large $B$ can be obtained from increasing the valley degeneracy $N_{\mathrm{v}}$, without direct effect on carrier mobility reduction from increasing of actual effective mass of mobile in the energy band. Note that there is still a reduction effect due to intervalley scattering when the carriers move across the valley to the other one.

Currently there are two ways to get large valley degeneracy. First, from intrinsic properties of the material, i.e., materials that have high symmetry will yield large valley degeneracy. ${ }^{3}$ If the energy bands extrema (bottom of valence bands or top of conduction bands) of those materials fall on the high symmetry point in Brillouin zone, it will give large valley degeneracy. But if the extrema of the materials fall on the $\Gamma$-point, the parameter $B$ will be not improved, since $\Gamma$-point will yield only one valley degeneracy. The highest symmetry structure is cubic, which has 48 symmetries, followed by hexagonal groups of 24 symmetries, etc. ${ }^{3}$ This suggests that the best thermoelectric materials need to be an indirect band gap semiconductor with cubic structure like $\mathrm{PbTe} .{ }^{22,34,84,88}$

Second, the convergence of electronic bands approach, ${ }^{83,91-93}$ it can be considered when two bands or more than two (mostly first and second extrema bands) at different point or the same point in Brillouin zone converge into each other yielding degenerate band (large valley degeneracy). This convergence of the energy bands can be facilitated by alloying, resulting in an improvement of $Z T .{ }^{91,93}$ The main reason on this high valley degeneracy can improve the over all $Z T$ is that when two bands are converge to each other leading to rapid increase in electronic density of state similar to the energy band distortion. Thus the Seebeck coefficient is enhanced by this fact. Since carriers in the valley degeneracy approach move in their own band without any energy band distortion. Thus they do not increase an inertial effective mass resulting 
in an improvement of Seebeck coefficient. Pei et al. followed up this study by modified the material parameter. ${ }^{86,87,94}$ In this case, they considered that since the majority chemical bond in thermoelectric materials are covalent. Thus it is safe to assume that both non-polar acoustic and optical phonon-electron scattering is dominated for thermoelectric transport. The phonon-electron scattering mobility $\mu_{\text {el-ph }}$ is given ${ }^{46,94-96}$ by

$$
\mu_{\text {el-ph }}=\frac{2^{3 / 2} \pi^{1 / 2} \hbar^{4} e C_{l}}{3 m_{c}^{*}\left(m_{b}^{*} k_{\mathrm{B}} T\right)^{3 / 2} D_{\text {el-ph }}^{2}},
$$

where $e$ is a electron charge, $N_{\mathrm{v}}$ is the valley degeneracy, $C_{l}$ is the average elastic constants, $D_{\mathrm{el} \text {-ph }}$ is the deformation potential coefficient, $\kappa_{l}$ is the lattice thermal conductivity, $T$ is the absolute temperature, $m_{c}^{*}$ is the carrier conductivity effective mass, and $m_{b}^{*}$ is the band effective mass. If we assume isotropic carrier pocket meaning $m_{1}^{*}=m_{2}^{*}=m_{3}^{*}$ due to isotropic carrier pocket has similar band curvature. This assumption give $m_{c}^{*}=m_{b}^{*}$. Then we can rewrite the material parameter as

$$
B_{\mathrm{mod}}=\frac{2 k_{\mathrm{B}}{ }^{2} \hbar}{3 \pi} \frac{N_{\mathrm{v}} C_{l}}{m_{c}^{*} D_{\text {el-ph }}^{2} \kappa_{l}} T .
$$

From this equation, it suggests that good thermoelectric material should have low lattice thermal conductivity, low effective mass, high valley degeneracy and weak coupling between charge carrier and phonon (low $D_{\text {el-ph }}$ ). Pei et al., showed that in PbTe doped I and La system. ${ }^{86}$ The band effective mass in PbTe:La is $20 \%$ heavier than PbTe:I due to increasing of the energy band gap resulting in band flattening. Their results showed that PbTe:La has higher Seebeck coefficient but PbTe:I exhibit superior in overall $Z T$ in entire temperature range, since the heavy band effective mass reduces carrier mobility in PbTe:La system. According to Pei et al. deformation potential coefficient $D_{\text {el-ph }}$ reduction is another way to increase $Z T$ of materials. There are a few researches showed that the $D_{\text {el-ph }}$ can be lower in the low dimension materials like thin films due to the biaxial strain between film and substrate.

In addition Jeong et al. found similar conclusion on their theoretical investigations on the best bandstructure for thermoelectric. ${ }^{97}$ Basically they followed the Mahan and Sofo study, but they used Landauer formula which is a simplify version of Boltzmann transport equation (BTE) to explain the carrier transport instead of using BTE. In their investigations, they considered the effect 
of different scattering mechanism. Their results suggested that the optimum bandstructure is sensitive to the scattering mechanism. If the carriers relaxation time is independent with energy (constant relaxation time), they arrive at the same conclusion as Mahan and Sofo, i.e., asymmetry sharp peak on electronic DOS around the Fermi level leads to maximize power factor $S^{2} \sigma$.

On the other hand, the board feature on the electronic DOS will give better improvement of power factor compare to narrow feature on the electronic DOS, if the relaxation time is proportional to the density of states $\tau(E)=\frac{C_{\mathrm{el}}}{D(E)}$ where $C_{\mathrm{el}}$ is constant. Because the electronic DOS with a very narrow feature around Fermi level will rapidly increase as energy increase resulting a dramatically decrease on electrical conductivity due to short relaxation time (high scattering rate). So we are back to our old dilemma, an increasing of Seebeck coefficient leads to reduction in electrical conductivity or vice versa. Actually, the scattering mechanism that we consider is a charge carrier-non polar acoustic phonon scattering or charge carrier-optical phonon scattering, similar to the scattering mechanism that was considered by Pei et al. ${ }^{86,87,94}$. Since the scattering relaxation times of these scattering mechanisms are inversely proportional to electronic DOS (see ref.[46, 96] for exact formula).

Furthermore, Jeong et al. also made a comparison study between high valley degeneracy and resonant energy level that distort electronic DOS approach, they concluded that the high valley degeneracy is better approach to improve the power factor than the resonant energy level electronic DOS distortion approach when the relaxation time is inversely proportional to density of state. They found that the electronic DOS distortion approach can indeed enhance the Seebeck coefficient, but it also degrades the electrical conductivity with the same reason that we discussed above. This is similar to Pei et al. comment on their review article about resonant energy level. ${ }^{87}$

Nevertheless, there are still possibilities to improve power factor in the resonant energy level that distorts DOS approach. Jeong et al. observed that electrical conductivity in the resonant energy level that distort DOS approach can be increase when Fermi level near DOS distortion, ${ }^{97}$ thus both Seebeck coefficient and electrical can be improved. The idea of co-doping that one doping con- 
tribute DOS distortion and another doping adjust the Fermi level to improve power factor was also suggested by Pei et al. ${ }^{87}$

Note that these considerations are based on two main assumptions i.e., non-polar phonon carrier scattering and isotropic band. In reality, it may not be isotopic as the assumption state. Pei et al., suggested we should optimize transport properties on the lightest band since it give lowest inertial effective mass, ${ }^{87}$ whereas Jeong et al., suggested that the degree of anisotropic of inertial effective mass and increase valley degeneracy can improve the thermoelectric properties. ${ }^{97}$ And they also stated that in mulitband semiconductor the conductivity depends strongly on valley degeneracy than on the degree of anisotropy. In addition, it is indeed safe to say that the non-polar phonon carrier scattering dominate. However, there are difference scattering mechanisms which give different band effective mass dependent. The electron-ionized impurity relaxation time is proportional to $\left(m_{b}^{*}\right)^{1 / 2},{ }^{46,96}$ whereas polar optical phonon-electrons or piezoelectric relaxation time is depended on $\left(m_{b}^{*}\right)^{-1 / 2} .46,96$ So these scattering mechanism will change the relation between $\mu$ and $m_{d}^{*}$. Thus one need to be careful when applied this approach. Since heavily doped or Polar materials that consist of some degree of electric dipole moment from the chemical bond can change the scattering mechanism of electrons.

\subsubsection{Optimum band gap of thermoelectric materials}

Apart from introducing a material parameter, Chasmar and Stratton also suggested the optimum band gap for the best thermoelectric materials. ${ }^{85}$ They discussed that at high temperature, minority carriers will decrease the Seebeck coefficient and increase the electronic thermal conductivity. This effect is called bipolar effect which is directly depended on the band gap of the materials. A small band gap requires low thermal energy to generate minority carrier (intrinsic regime) causing large bipolar effect. However, a large band gap is also not preferable, since it has too low carrier concentration and typically high lattice thermal conductivity. Therefore, they suggested the optimum of thermoelectric materials to be $6 k_{\mathrm{B}} T$, about $0.025 \mathrm{eV}$ at $\mathrm{T}=300 \mathrm{~K}$ and $0.070 \mathrm{eV}$ at $\mathrm{T}=$ $800 \mathrm{~K} .{ }^{85}$ Later, Mahan and Sofo generalized Chasmar and Stratton's approach by considering the case of partially or completely degenerate semiconductors and introducing new optimized pa- 
rameters for maximizing $Z T .{ }^{98,99}$ They emphasized the importance of the material parameter $B$ in that the $Z T$ is increased when $B$ increases and showed that the $Z T$ also depends on the materials parameter $B$ for degenerate semiconductor. But the $Z T$ will be larger when the material is less degenerate. Furthermore, they suggested that for the best band gap of semiconductor for large $Z T$ should equal to $10 k_{\mathrm{B}} T$ for both direct and indirect band gap, which is higher than suggestion from Chasmar and Stratton.

Inspired by the fact that the maximum of $Z T$ is limited by $B$ where it depends on the effective mass. Therefore, Mahan and Sofo reconsidered their approach by claiming that if the effective mass is proportional to the band gap. ${ }^{99}$ They concluded that there are two regions in the behavior of $Z T$ as a function of the band gap. For the band gap below $6 k_{\mathrm{B}} T$, the $Z T$ will decrease with decreasing the band gap because the bipolar effect, in agreement with Chasmar and Stratton. For the band gap higher than $10 k_{\mathrm{B}} T$, the $Z T$ will increase or decrease with the band gap depending on the dominate charge scattering mechanism which affects the band effective mass. For the case of acoustic phonon or polar optical phonon charge carrier scattering, the optimum band is $10 k_{\mathrm{B}} T$. And if the ionization scattering dominates instead, there is no optimum due to the material parameter $B$ increase with band gap.

Yet, there are some way to overcome the effect of bipolar effect, forming a solid solution with the larger band gap material can result in an increase of the band gap or utilizing the heterostructure band offsets between nanocomposite and matrix that can scatter electrons and holes differently. These two ideas can help us improve the $Z T$ of small band gap material or shifted the $Z T$ peak to higher temperature thus we are possible to use the materials at high temperature. 


\subsubsection{Energy filtering approach}

To consider the idea of energy filtering, let us recall the electrical conductivity and Seebeck coefficient expression (Equation (3.41) and (3.42)) :

$$
\begin{aligned}
\sigma & =\frac{1}{\rho}=\int \Sigma(E)\left(-\frac{\partial f_{0}}{\partial E}\right) d E \\
S & =\frac{1}{q T} \frac{\int \Sigma(E)\left(E-E_{F}\right)\left(-\frac{\partial f_{0}}{\partial E}\right) d E}{\int \Sigma(E)\left(-\frac{\partial f_{0}}{\partial E}\right) d E} \\
& =\frac{\left\langle E-E_{F}\right\rangle}{q T} .
\end{aligned}
$$

We can consider Seebeck coefficient as the entropy carried by the charge carriers. Or we can use Thomson relation $\pi=T S$, so the Peltier coefficient is the average energy transported by the change carriers. This suggest that if we have a lot of charge carriers that carry very energy above Fermi level, the highest Seebeck coefficient should be obtained. So how to do that, since Seebeck coefficient depends on Fermi window function $\left(-\frac{\partial f_{0}}{\partial E}\right)$ and transport distribution function $\Sigma(E)$ which is related to both relaxation time and electronic density of state (see Equation (3.44)). Since the number of charge carriers that participate in the conduction is given by Fermi window function which has a Gaussian-shape function centered at $E=E_{F}$ and a width of $\sim k_{\mathrm{B}} T$. Thus if the electronic DOS has asymmetric shape at the Fermi level meaning that the system will have more high energy electronic states for charge carriers. This should result in an increasing of Seebeck coefficient because there are more high energy charge carriers in the conduction. Thus, an asymmetric electronic DOS acts as a energy filler that fillers the low energy charge carrier and allow only high energy charge carriers can pass through.

And to maximize electrical conductivity, the transport distribution function need to be large within Fermi window so that electrical conductivity can be maximized. Because of this energy filtering effect from asymmetric, narrow and high electronic DOS, high power factor can be obtained. This is qualitative explanation why Mahan and Sofo bandstructure can improve the power factor. ${ }^{6}$ However if we create this asymmetry shape feature on electronic DOS by energy band distortion, it may lead to electrical conductivity compensation. Thus there is another way to obtain the effect of 
energy filtering. Since the charge carrier can be selectively filtered by the use of tall barriers $\left(\sim 1-10 k_{\mathrm{B}} T\right)$, we can get these barriers from heterostructure (single barrier) or superlattice (multibarriers). ${ }^{6}$ These tall barriers cut the contribution from low energy carriers in the conduction since they cannot move across or tunneling through the barriers. This effect gives the shape of transport distribution function (the product of density of states and Fermi-Dirac distribution) becoming asymmetric due to the modification of Gaussian-shape of Fermi window function. Therefore the high Seebeck coefficient can be obtained. We call this idea as the distortion of the electronic density of states through charge carrier energy filtering. ${ }^{6,100-102}$ However, the electrical conductivity of this structure will decrease. Because of a few high energy electrons/holes that move along in direction that perpendicular to the barrier can be emitted into structure. Electrons/holes that move in transverse direction cannot pass through the barrier although they have energy higher than the barrier height due to the conservation of transverse momentum causing a reverse current (electrons backscattering at barrier). The rough surface or scattering centers needs to be introduced in each layer of superlattice to make barriers are non-planar which will facilitate more high energy electrons/holes can be emitted.

Zide et al. demonstrated this approach in InGaAs/InGaAlAs superlattice show that the Seebeck coefficient is improved by factor of 2 to $3{ }^{103-105}$ Introducing those barriers will, however, decrease charge carrier mobility yielding low electrical conductivity. Therefore, the idea of metal/semiconductor was introduced to increase amount of conduction carrier. This was predicted in $\mathrm{ZrN} / \mathrm{ScN}$ superlattices, the calculated $Z T$ shows that it can be as large as 3 at $1200 \mathrm{~K} .{ }^{106}$ According to experimental study of similar structure $\mathrm{ZrN}(4 n \mathrm{~m}) / \mathrm{ScN}(6 n \mathrm{~m})$ superlattices claimed to show a room temperature Seebeck coefficient of $840 \mu \mathrm{VK}^{-1} .{ }^{6}$

\subsubsection{Modulation doping}

Inspirit by the fact that charge carrier can be scattered by ionized donors, acceptors or impurities, according to the relaxation time of ionized impurity scattering that is given ${ }^{46,95}$ by

$$
\tau_{I}(\xi)=\frac{16 \sqrt{2} \pi \epsilon^{2}\left(m_{b}^{*}\right)^{1 / 2} \xi^{3 / 2}}{N_{I} Z^{2} e^{4}\left[\ln \left(1+\gamma^{\prime}\right)-\gamma / 1+\gamma^{\prime}\right]}
$$


where $N_{I}$ is the number of ionized impurities, $Z$ is the atomic number, $e$ is the electronic charge, $\epsilon$ is the dielectric constant, $m_{b}^{*}$ is the density of state effective mass, $\xi$ is the reduced carrier energy $\xi=\frac{E}{k_{\mathrm{B}} T}$ and $\gamma^{\prime}=8 \epsilon m_{b}^{*}\left(\frac{k_{\mathrm{B}} T E}{\hbar^{2} q^{2} n}\right)$ where $n$ is the carrier concentration.

We can see that more dopants lead to low relaxation time (high scattering rate). In order to doped our thermoelectric material efficiently, the modulation doping was utilized. The modulation doping is not a brand new idea. The technique has been used in thin-film heterostructure as a type of high-electron-mobility transistor structure so called Modulation Field Effect Transistor (MODFET). ${ }^{107,108}$ Recently Zebarjadi et al. ${ }^{109}$ has implement similar idea of modulation doping in the nanocomposite bulk sample where doped nanoinclusions were embedded inside the undoped matrix. In their study, they used $\mathrm{Si}_{0.8} \mathrm{Ge}_{0.2}$ as matrix and $\mathrm{Si}_{0.95} \mathrm{~B}_{0.05}$ or $\mathrm{Si}_{0.97} \mathrm{P}_{0.03}$ as doped nanoinclusions. By grouped up dopants in the nanoinclusions, charge carriers that are injected from doped nanoinclusions due to the band alignment can now move freely in the undoped matrix. Thus the power factor can be improved due to increase of electrical conductivity compared to the convectional doping material with the same amount of doping concentration. If we combine this concept with the nanostructuring all-scale hierarchical architecting that discussed in Section (3.4.1.4) to engineer thermoelectric material. The maximum $Z T$ material should be obtained. After this idea was brought up there are serval report show that they can obtain the similar tend. $58,110,111$

Moreover, Faleev and Leonard mathematically showed that the Seebeck coefficient can be improved by metallic nanoinclusions in semiconductor host. ${ }^{112}$ The key mechanism is the driving force from non-equal Fermi level between metal and semiconductor causing electronic band bending (Schottky barrier) at interfaces. This barrier will not affect transportation of high energy charge carrier, however low energy charge carrier are strongly scattered. Thus this effect can give relaxation time have strong dependent with energy yielding enhancement of Seebeck coefficient (for example see Mott equation (3.58)). This concept can be considered as energy filtering enhance Seebeck coefficient instead of asymmetry electronic DOS, here we create asymmetry relaxation time via the Schottky barrier scattering to filter cold electron instead. 
This concept was brought up again in the review article by Yang et al. ${ }^{113}$ They suggested that we can utilize the grain boundary barrier to energy filter (scatter) low energy charge particle. Thus if we can combine this concept with modulation doping both Seebeck coefficient and electrical conductivity should improve.

\subsubsection{Reducing the electronic thermal conductivity}

Since the thermal conductivity contributed by electrons is small compare to the lattice thermal conductivity. Thus it is taken out when we consider the engineering strategy improve $Z T$. But all the attempt that we put on the engineer our material to obtain as high as possible $Z T$ involve with increasing electrical conductivity and reduce lattice thermal conductivity in the end the electron thermal conductivity will be dominate. In this fashion, if one could come up with an concept to reduce electron thermal conductivity without reducing electrical conductivity, this concept would be ideal for improving $Z T$. To find such a possibility let us consider the Seebeck coefficient $S$ (Equation (3.41)) and electron thermal conductivity $\kappa_{e}$ via the short-circuit thermal conductivity $\kappa_{0}$ (Equation (3.43)), then rewritten them in term of energy average

$$
S=\left(\frac{k_{\mathrm{B}}}{q}\right)\left\langle\left(\frac{E-E_{F}}{k_{\mathrm{B}} T}\right)\right\rangle
$$

and

$$
\kappa_{0}=\left(\frac{k_{\mathrm{B}}}{q}\right)^{2}\left\langle\left(\frac{E-E_{F}}{k_{\mathrm{B}} T}\right)^{2}\right\rangle T \sigma,
$$

where the average term is given by

$$
\left\langle\left(\frac{E-E_{F}}{k_{\mathrm{B}} T}\right)\right\rangle=\frac{\int \Sigma(E) \frac{\left(E-E_{F}\right)}{k_{\mathrm{B}} T}\left(-\frac{\partial f_{0}}{\partial E}\right) d E}{\int \Sigma(E)\left(-\frac{\partial f_{0}}{\partial E}\right) d E},
$$

where $\Sigma(E)$ is the transport distribution function. Equation (3.44). Substituting these expression for Seebeck coefficient and short circuit thermal conductivity into Equation (3.43) and rewrite them in term of electron thermal conductivity $\kappa_{e}$, we get

$$
\begin{aligned}
\kappa_{e} & =T \sigma\left(\frac{k_{\mathrm{B}}}{q}\right)^{2}\left\{\left\langle\left(\frac{E-E_{F}}{k_{\mathrm{B}} T}\right)^{2}\right\rangle-\left\langle\left(\frac{E-E_{F}}{k_{\mathrm{B}} T}\right)\right\rangle^{2}\right\} \\
& =T \sigma \mathcal{L} .
\end{aligned}
$$


Here the $\mathcal{L}$ is called Lorentz number, and it can be written as

$$
\mathcal{L}=\left\langle\left(\frac{E-E_{F}}{k_{\mathrm{B}} T}\right)^{2}\right\rangle-\left\langle\left(\frac{E-E_{F}}{k_{\mathrm{B}} T}\right)\right\rangle^{2} .
$$

This shows that the Lorenz number is no longer a constant as stated by Wiedemann-Franz law i.e., $\mathcal{L} \approx 2\left(\frac{k_{\mathrm{B}}}{q}\right)^{2}$ for nondegenerate 3D semiconductors and $\mathcal{L} \approx\left(\frac{\pi^{2}}{3}\right)\left(\frac{k_{\mathrm{B}}}{q}\right)^{2}$ for fully degenerate (3D metals). The constant Lorentz number is just an approximation when consider the special case where the electronic band structure is parabolic and a energy independent electron relaxation time. Now the Lorentz number is depended on the details electronic band structure, charge carriers scattering mechanism, and degree of degeneracy for each material. This suggests that we can reduce electron thermal conductivity via Lorentz number tuning.

Actually, Mahan and Sofo was suggested the way to reduce electron thermal conductivity. The shape feature close to Dirac delta function of the a transport distribution function $\Sigma(E)$ Equation (3.44) can reduce electron thermal conductivity. This also confirmed by Jeong et al. ${ }^{77,97}$ Note that Mahan and Sofo considered the charge carrier transport with constant relaxation time. To find materials that have electronic DOS suggested by Mahan and Sofo seem to be not easy, since it required to have integrated background area under the electronic DOS less than $1 \% .{ }^{77}$ Nevertheless, there are other ways to reduce electron thermal conductivity via Lorentz number reduction. Bian et al. showed that the Lorentz number in the superlattices can be tailored by varying the doping concentration and layer thickness. They showed that in some condition the $50 \%$ reduction of Lorentz number can be obtained. ${ }^{114}$ There was theoretical study by Mukerjee and Moore ${ }^{115}$ in the strongly correlated materials $\mathrm{Na}_{x} \mathrm{CoO}_{2}$ suggested that the Wiedemann-Franz law does not hold due to the uncorrelated assumption in the Wiedemann-Franz law.

As one can see that the maximizing power factor is still a challenging topic compared to reducing thermal conductivity, due to the trade-off between Seebeck coefficient and electrical conductivity. Thus, the main gain of $Z T$ at current stage comes from the reduction of lattice thermal conductivity. This means that we need a new approach that can couple maximize of power factor 
and reduction of lattice thermal conductivity as shown in PGEC concept. These presented approaches have shown that they can couple between maximizing power factor and also reduce the lattice thermal conductivity due to the alloying, nanoinclusion, or superlattice.

\subsection{Review of scandium nitride $(\mathrm{ScN})$}

$\mathrm{ScN}$ is an interstitial transition-metal nitride, metal atoms form a close-pack structure with nitrogen atoms occupy octahedral sites. Thus, ScN possesses a B1 $(\mathrm{NaCl})$ crystal structure, two interleaved of Sc and N with lattice parameter $a=4.501 \AA$. This follows the empirical prediction by Hägg which stated that if the ratio of the radii between non-metal to metal atoms, $r_{x} / r_{m}$ is smaller than 0.59 , the structure can be bcc, fcc, or hcp lattices. ${ }^{116}$ Like other transition-metal nitride, ScN has excellent mechanical and electrical properties that suitable for mid temperature thermoelectric application (400-700 ${ }^{\circ} \mathrm{C}$ ). ScN has high hardness, $\mathrm{H} \sim 21 G \mathrm{~Pa}$, chemically inert for most acid and bases, and high temperature stability with a melting point, $T_{m} \sim 2550{ }^{\circ} \mathrm{C} .{ }^{117-119}$ In addition, $\mathrm{ScN}$ is stable in air up to $550{ }^{\circ} \mathrm{C}$ (note that,there is $1-2 \mathrm{~nm}$ of surface oxide) and oxidized to $\mathrm{Sc}_{2} \mathrm{O}_{3}$ when temperature above 600 ${ }^{\circ} \mathrm{C} .{ }^{119-121}$ Hall measurements on as-deposited ScN showed n-type semiconductor with the carrier concentration of $\mathrm{ScN}$ has been reported to vary from $10^{18}$ to $10^{22} \mathrm{~cm}^{-3}$ due to incorporated impurities such as halogens, oxygen or $\mathrm{N}$ vacancies during synthesis and electron mobility of $1-1.8 \mathrm{~m}^{2} \mathrm{~V}^{-1} \mathrm{~s}^{-1} \cdot{ }^{119,121-126}$

Apart from these properties, most of the investigations on $\mathrm{ScN}$ concentrate on its electronic structure of $\mathrm{ScN}$, discussing whether $\mathrm{ScN}$ is a semimetal or semiconductor. ${ }^{121,122,125-128}$ Because of it is difficult to obtain pure $\mathrm{ScN}$, for example the report from Moram et al. shows that $\mathrm{ScN}$ has higher affinity to oxygen than TiN or ZrN. ${ }^{129}$ Therefore, those free carriers from impurity gives an uncertainty of the band gap determination by optical technique due to Burstein-Moss shift leading misinterpretation of band gap in ScN. ${ }^{121,125}$ Moreover, the theoretical calculation has shown underestimation of the band gap (detail discussion in Chapter 4) leading to the idea of $\mathrm{ScN}$ is a semimetal. The results of studies show that $\mathrm{ScN}$ is indirect semiconductor with band gap of in a range of $0.90-1.6 \mathrm{eV} .^{122,127}$ 
Recently, Deng et al. reported experimental and theoretical investigations on $\mathrm{ScN}$ electronic structure and transport properties. ${ }^{130}$ They found that the amount fluorine impurities in their ScN samples decreases with the Sc target lifetime. They states that this $\mathrm{F}$ comes from the purification process by fluorine reduction. They also stated that the found a trace about of Ta in their sample as well. Due to these F and Ta leads to carrier concentration in different ScN samples varies from 12.8 to $1.13 \times 10^{20} \mathrm{~cm}^{-3}$ with a related amount of $\mathrm{F}$ from $3 \pm 1$ to $<0.5$ at.\%. Based on this finding and the optical properties measurement, they confirmed that the optical properties of $\mathrm{ScN}$ is affected by this carrier concentration according to Burstein-Moss shift. Furthermore, they calculate the electronic structure of ScN with density functional theory. Here they applied HSE06 hybrid functional as their exchargecorrelation energy. They justify their results based on the optical properties that extracted from the results of calculations compare with the experimental results. They confirmed that $\mathrm{ScN}$ is a indirect semiconductor with band gap of $0.92 \pm 0.05 \mathrm{eV}$ at $\Gamma \rightarrow X$. Together with this results, they reported the transport effective mass at $X$ point $m_{t r}^{*}=0.40 \pm 0.02 m_{e}$ and density of state effective mass at $X$ point $m_{\mathrm{DOS}}^{*}=0.33 \pm 0.02 m_{e}$ where $m_{e}$ is the mass of electron. Since $m_{t r}^{*} \neq m_{\text {Dos }}^{*}$ suggests that the conduction band carrier pocket (band valley) at $X$ point is anisotropy.

ScN has been synthesized in thin films form, starting in early of 1970. Dismukes et al. reported that the polycrystalline ScN thin film with films thickness up to $20 \mu \mathrm{m}$ can be grown on $\alpha-\mathrm{Al}_{2} \mathrm{O}_{3}(1 \overline{1} 02)$ (r-plane sapphire) by halide chemical vapor deposition technique at $850-1000{ }^{\circ} \mathrm{C} .{ }^{121}$ Later they showed that $\mathrm{ScN}$ could epitaxially growth on $\mathrm{Al}_{2} \mathrm{O}_{3}(0001)$ and $\mathrm{Al}_{2} \mathrm{O}_{3}(1 \overline{1} 02){ }^{124}$ However, ScN film that is grown by this technique results in incorporation of halogen impurities. ${ }^{121,124}$

In order to avoid halogen incorporation, Gall et al. used the reactive magnetron sputtering technique to grow $\mathrm{ScN}$ in $\mathrm{N}_{2}$ atmosphere under ultrahigh vacuum conduction. ${ }^{117}$ The results of this study shows that the polycrystalline $\mathrm{ScN}$ thin films can be growth on $\mathrm{MgO}(001)$ at growth temperature about $750{ }^{\circ} \mathrm{C}$. The mix orientation of 111 and 002 of ScN occurs due to the growth kinetic that limited by the diffusivity of Sc adatom. In order to obtain a single crystal, TiN seed layer has been used to increase Sc adatom 
mobility. ${ }^{131}$ Furthermore, the high energy ion bombardment via unbalance magnetron and substrate bias technique had been used to increase Sc adatom mobility. ${ }^{117}$ The result shows that they can obtain single-crystal epitaxial growth $\mathrm{ScN}$ thin films by using $\mathrm{N}_{2}^{+}$ energy of $20 \mathrm{eV}$ at $750{ }^{\circ} \mathrm{C}$ for growth temperature without any seed layer. Moreover, the result from Rutherford backscattering spectroscopy (RBS) shows that their ScN films have N/Sc ratios of $1.00 \pm 0.02$. Recently work on sputtering by Gregoire et al. shows that single crystal $\mathrm{ScN}$ can be grown on $\mathrm{Al}_{2} \mathrm{O}_{3}(1 \overline{1} 02)$ with $20 \%$ of $\mathrm{N}_{2}$ in Ar ambient at growth temperature of $820{ }^{\circ} \mathrm{C} .{ }^{132}$ They also show that the film structure and electrical properties charge with the deposition geometry yielding they can obtain smooth surface and high change carrier mobility in $\mathrm{ScN}$ thin film.

Moustakas et al. showed that the stoichiometric polycrystalline ScN 111 orientation thin film can be grown on $\mathrm{Al}_{2} \mathrm{O}_{3}(0001)$ with an AlN seed layer by electron cyclotron resonance plasma-assisted molecular beam epitaxy (MBE). Al-Brithen et al. used radio frequency (RF) molecular beam epitaxy (MBE) to study Sc:N flux ratio on the growth mode and structure of $\mathrm{ScN}$ thin film. ${ }^{122,123,133}$ They showed that at Sc-rich regime, Sc-Sc bonds are formed at film surface lead to low diffusion barrier because this bond was weaker than Sc-N bond. Therefore, the surface adatoms diffusivity is higher than $\mathrm{N}_{2}$-rich regime yielding a flat-plateaus surface. Morem et al. studied MBE deposition of 111 oriented ScN on Si (111). ${ }^{134}$ They show that the quality of $\mathrm{ScN}$ film depends on the growth temperature. The highest $\mathrm{ScN}$ films quality on $\mathrm{Si}(111)$ was grown at optimum growth temperature of $850{ }^{\circ} \mathrm{C}$.

The above review showed that $\mathrm{ScN}$ is a cubic semiconductor with an indirect band gap at X point in Brillouin zone, which matches the requirement of high material parameter and optimum band gap for thermoelectric application. Also it has wide range of the carrier concentrations that span over the typical ideal range for thermoelectrics ${ }^{4}$ while it can possible to retaining a high carrier mobility. ${ }^{132}$ Moreover several investigations show that $\mathrm{ScN}$ can accumulate nitrogen vacancies or be introduced in a form of solid solution. Those vacancies and alloying atoms could yield an asymmetric feature at electronic density of states ${ }^{135}$ and also reduce lattice thermal conductivity in ScN. Furthermore, According to these reasons and the investigation of $\mathrm{ZrN} / \mathrm{ScN}$ superlattice by Zebarjadi et al. ${ }^{106}$ show that $\mathrm{ScN}$ has high potential to be a good 
thermoelectric material which results in high electrical conductivity coupled with large Seebeck coefficient yielding possibility to obtain large power factor. The results of my investigations are shown in [Paper I and II].

\subsection{Review of chromium nitride (CrN)}

$\mathrm{CrN}$ is a well-studied transition metal nitride. Most of the research on $\mathrm{CrN}$ have been related to it possible applications as a protective hard coating. For such purposes $\mathrm{CrN}$ has excellent mechanical properties such as high hardness ( $\sim 28 \mathrm{GPa}$ ), high corrosion resistance, and excellent wear properties. ${ }^{136-142} \mathrm{CrN}$ is easy to synthesis as a thin film by magnetron sputtering (DC, pulsed DC, middle frequency RF, or High Power Impulse). ${ }^{141-147} \mathrm{CrN}$ thin films can be grown with a very low internal stresses, thus it can be synthesized in various thickness from thin to thick coatings (100 $\mathrm{nm}$ to $100 \mu \mathrm{m}$ ) on a variety of steels and engineering ceramics with very good adhesion. ${ }^{137,141,142,148}$ Moreover there are number of reports on possibility on synthesis $\mathrm{CrN}$ in a bulk form as well. ${ }^{149-153}$ In addition to these properties, CrN exhibits a magnetostructural phase transition. ${ }^{154-156}$ At Néel temperature $\left(T_{\mathrm{N}}\right)$ above $270-286 \mathrm{~K}$ i.e., $\mathrm{CrN}$ possesses a paramagnetic $\mathrm{B} 1(\mathrm{NaCl})$ crystal structure with a lattice parameter reported to range from 4.135 to $4.185 \AA \AA^{143,157-160}$ When temperature below Néel temperature, it changes its phase to antiferromagnetic orthorhombic structure. Rivadulla et al., found that the pressure can induce phase transformation between paramagnetic CrN or antiferromagnetic $\mathrm{CrN}$ resulting the $T_{\mathrm{N}}$ is pressure dependent parameter. ${ }^{161}$ This result was later confirmed by Wang et al. For the high temperature phase, thin films of $\mathrm{CrN}$ rock-salt structure stable up to $600{ }^{\circ} \mathrm{C}$ when annealed in air before it decompose into $\beta-\mathrm{Cr}_{2} \mathrm{~N}$ which have a hexagonal phase, and later oxides at $700{ }^{\circ} \mathrm{C} .{ }^{146}$ Due to this transition, the drawback of using $\mathrm{CrN}$ for thermoelectric applications is the low thermal stability which limits usability at high temperature. Thus, $\mathrm{CrN}$ is engineered in order to be used at high temperature, a form of solid solution $\left(\mathrm{Cr}_{1-\mathrm{x}} \mathrm{Al} \mathrm{x}_{\mathrm{x}} \mathrm{N}\right)$ or superlattice (CrN/AlN, CrN/TiN, etc.) is chosen which has shown that they can improve $\mathrm{CrN}$ thermal stability as well as mechanical properties. ${ }^{136,147,162-169}$ 
Despite the fact that the mechanical properties of $\mathrm{CrN}$ has been well studied. The electrical properties of $\mathrm{CrN}$ are still controversial with conclusion varying from report to report. For example, many studies showed that the room temperature electrical resistivity of single crystal $\mathrm{CrN}$ is varied within a wide range of $1.7 \times 10^{-3}$ to $3.5 \times 10^{-1} \Omega \mathrm{cm} .{ }^{143,150-152,157,159,160,170,171}$ In addition, the reported temperature dependent electrical resistivity behavior upon the magnetostructural phase transition from orthorhombic to cubic rock-salt phase shows various of behaviors, metal to metal, ${ }^{159}$ metal-semiconductor, ${ }^{157}$ or semiconductor to semiconductor. ${ }^{149,151,171,172}$

In an early experimental work by Gall et al., ${ }^{170}$ it was observed that there is no cubic-to-orthorhimic phase transition occurring in their sample due to the epitaxial constrains in $\mathrm{CrN}_{1-\mathrm{x}}(001)$ film which $\mathrm{x} \leq 0.03$ on $\mathrm{MgO}(001)$ substrate. CrN films were grown by reactive DC magnetron sputtering. This result is in agreement with the report by by Inumaru et al., that showed the $\mathrm{CrN}(111)$ films grew on $\alpha-\mathrm{Al}_{2} \mathrm{O}_{3}$ does also not change their structure. ${ }^{159}$ For the electrical transport properties semiconductor behavior in wide temperature range from temperature below $T_{\mathrm{N}}$ to temperature above $T_{\mathrm{N}}$. In addition, Gall et al., also suggested that the conducting behavior in $\mathrm{CrN}$ films is a hopping conduction mechanism, and the band gap of $\mathrm{CrN}$ depends on the correlation energy drives the gap opening. ${ }^{170}$ Thus $\mathrm{CrN}$ could be a considered as a Mott insulator. On the contrary, Constantin et al., showed that their $\mathrm{CrN}(001)$ film on $\mathrm{MgO}(001)$ substrate grew by molecular beam epitaxy (MBE) has discontinuity appears on the temperature dependent electrical resistivity; ${ }^{157}$ it changes from metal for the temperature below $T_{\mathrm{N}}$ to semiconductor at temperature above $T_{\mathrm{N}}$. In this case they also calculated the band gap of $\mathrm{CrN}$ rock-salt phase which equal to $0.07 \mathrm{eV}{ }^{157}$

On the theoretical side, Herwadkar et al., ${ }^{173}$ performed density functional calculation with local density approximation corrected by Hubbard Coulomb terms ( $\mathrm{LDA}+\mathrm{U}$ ) where $U$ is varied from 3 to 5 for the $d$ electrons in $\mathrm{Cr}$ atoms. Their study focused on the low-temperature antiferromagnetic phase. The results suggested that $\mathrm{CrN}$ is a semiconductor at low temperature due to there is a band gap in electronic density of state at $U=3 \mathrm{eV}$. Here, they found that the band gap of $\mathrm{CrN}$ antiferromagnetic phase rather small, slightly below $1 \mathrm{eV}$ for the band gap. ${ }^{173}$ This small band 
gap and the fact that there are $\mathrm{N}$ vacancies may lead to a metalliclike behavior conduction at temperature below $T_{\mathrm{N}}$ due to electron doping from $\mathrm{N}$ vacancies. In addition, they commented that the $\mathrm{CrN}$ is a charge transfer insulator rather than Mott insulator due to the top of the valence band dominates by anion $p$ states and bottom of conduction band dominates by $d$ states. ${ }^{173}$

For the paramagnetic phase of $\mathrm{CrN}$, Alling et al., ${ }^{174}$ developed a theoretical description to simulate the magnetic disorder in paramagnetic phase of CrN. In their study, they utilized the special quasirandom structures (SQS) which is usually used to model disordered alloy structure (see Section (4.6)). Instead, they used it to model the magnetic disorder of $\mathrm{Cr}$ atoms in $\mathrm{CrN}$ paramagnetic phase. They assume 50\%-50\% concentration between $\mathrm{Cr}^{\uparrow}$ and $\mathrm{Cr}^{\downarrow}$ forming $\mathrm{Cr}_{0.5}^{\uparrow} \mathrm{Cr}_{0.5}^{\downarrow} \mathrm{N}$ paramagnetic phase meaning the total magnetic moment should equal to zero, and the magnetic pair correlation functions also equals zero on several coordination shells, which mimics an ideal paramagnet, but contrasting from e.g. ferromagnetic and antiferromagnetic states . This study Alling et al., suggested that $U=3 \mathrm{eV}$ is suitable for CrN paramagnetic phase. ${ }^{174} \mathrm{~A}$ result obtained by comparing the calculated density of state with the experimental photoemission data as well as the calculated structural parameters and experimental structure values. Alling et al., showed that a good description of the structural parameters of $\mathrm{CrN},{ }^{175}$ including the bulk modulus, demanded the consideration of finite local Cr magnetic moments, also in the high temperature paramagnetic phase, and not only in the low temperature antiferromagnetic phase. The most striking difference between non-magnetic and disordered magnetic modeling of the paramagnetic phase is perhaps seen for the bulk modulus. Rivadulla et al., suggested a collapse of the bulk modulus upon the transition from paramagnetic phase to antiferromagnetic phase ${ }^{161}$ while Alling et al., questioned the existence of such a collapse. ${ }^{175}$ Wang et al., later confirmed the view of Alling et al. ${ }^{153}$ Thus, I used this concept to model magnetic disorder in $\mathrm{Cr}_{1-\mathrm{x}} \mathrm{Sc}_{\mathrm{x}} \mathrm{N}$ my study.

In 2011, Gall et al. revisited this issue and conduct a series of investigations on electrical properties of $\mathrm{CrN}$ thin films growth by DC magnetron sputtering. From their studies, they confirmed their observation that the epitaxy can suppress the phase transition in $\mathrm{CrN}$ at $\mathrm{T}=T_{\mathrm{N}} \cdot{ }^{171}$ Here they compared between non- 
epitaxy polycrystalline CrN films on quartz glass with epitaxy single crystalline $\mathrm{CrN}(001)$ and $\mathrm{CrN}(111)$ on $\mathrm{MgO}(001)$ and $\mathrm{MgO}(111)$, respectively. Their results clearly showed that there is phase transition from low temperature antiferromagnetic phase to high temperature paramagnetic phase on polycrystalline samples but not on both single crystalline samples. In addition, they also did optical analysis on $\mathrm{CrN}(001)$ films on $\mathrm{MgO}(001)$. They suggested $\mathrm{CrN}$ has an indirect band gap of $0.19 \pm 0.46 \mathrm{eV}^{176}$ which is higher than the $0.09 \mathrm{eV}$ band gap that obtained from resistivity measurement by Herle et al., ${ }^{149}$ and $0.07 \mathrm{eV}$ suggested by Constantin et al. ${ }^{157}$

In addition, Gall et al. found that the room temperature electrical resistivity of $\mathrm{CrN}$ decreases as increasing crystalline quality of CrN. ${ }^{160}$ The conduction in $\mathrm{CrN}$ is govern by thermal activation of change carrier to the mobility edge in the upper Hubbard band. At the low temperature ( $\mathrm{T}$ below $130 \mathrm{~K}$ ), the electrical conduction of $\mathrm{CrN}$ can be described by the variable range hopping by Mott and Efors-Shklovskii (ES) with a cross over at $30 \pm 10 \mathrm{~K}$ from ES to Mott. Because of the number of $\mathrm{N}$ vacancies provide pathway for electron to hop at low temperature. On the other hand the crystal defects can cause the localized and delocalized states in the band tail of upper Hubbard band reduced the thermal activation energy to populate electron from Fermi level to the mobility edge. These $\mathrm{N}$ vacancies and crystalline defects can be generate as results from synthesis technique since $\mathrm{CrN}$ is sensitive to the high temperature due to phase decomposition as we discussed above; and the $\mathrm{N}_{2}$ can effect the N stoichiometry in CrN. Thus Gall et al. concluded that both $\mathrm{N}$ vacancies and the density of crystalline defects that can generate during synthesis are the main reasons for the variation of electrical transport in $\mathrm{CrN} .{ }^{160}$

These suggestions are confirmed by the recent report from Rivadulla et al. ${ }^{144}$ They studies thermoelectric effect of non-stoichiometric and stoichiometric $\mathrm{CrN}(001)$ thin films on $\mathrm{MgO}(001)$ growth by DC magnetron sputtering and annealed in ammonia $\left(\mathrm{NH}_{3}\right)$ gas for $2 \mathrm{~h}$ at $800{ }^{\circ} \mathrm{C}$ to obtain stoichiometric $\mathrm{CrN}$ films. The results shows that there is a factor of 100 different in the electrical resistivity. The main reason of such a improvement is due to improvement of crystalline quality yielding an improvement of mobility. ${ }^{144}$ Moreover the electrical transport behavior of show a metallic behavior for entire of temperature range with a kink 
at $T_{\mathrm{N}}$, for stoichiometric film. While the electrical transport behavior shows semiconductor behavior in entire of temperature range, for non-stoichiometric film. In both case, there is a kink appear at $T_{\mathrm{N}}$ indicating magnetostrutural phase transition transformation. For thermoelectric properties, there are some reports on bulk CrN showed that it has a large Seebeck coefficient of $135 \mu \mathrm{VK}^{-1}$ at room temperature up to $-200 \mu \mathrm{VK}^{-1}$ at $600 \mathrm{~K}$. And $\mathrm{CrN}$ has quite low thermal conductivity of about $1.7 \mathrm{Wm}^{-1} \mathrm{~K}^{-1}$ from room temperature to $600 \mathrm{~K}$. However, $\mathrm{CrN}$ has the drawback of relatively high electrical resistivity of $2 \times 10^{-5}$ to $1 \times 10^{-3} \Omega \mathrm{m}$ at room temperature. This is because the characteristically localized $3 d$ orbitals in CrN give a large effective mass for electrons resulting in large Seebeck coefficient and high electrical resistivity. Also the effect of impurities and crystal quality can increase the resistively. But the recently report from Rivadulla et al., shows that after adjusted the stoichiometry of $\mathrm{CrN},{ }^{144}$ the electrical resistivity reduces from $\sim 15 \mathrm{~m} \Omega \mathrm{cm}$ to $\sim 0.8 \mathrm{~m} \Omega \mathrm{cm}$ at T $=325 \mathrm{~K}$. Also they observe the improvement of Seebeck coefficient from $-75 \mu \mathrm{VK}^{-1}$ to $110 \mu \mathrm{VK}^{-1}$ at $\mathrm{T}=325 \mathrm{~K}$. While the lattice thermal conductivity is fairly low about $3 \mathrm{Wm}^{-1} \mathrm{~K}^{-1}$ at room temperature. From all these values result in $Z T$ of $\mathrm{CrN}$ thin film of about 0.16 at $\mathrm{T}=325 \mathrm{~K}$. Moreover there are some report on engineering CrN by forming solid solution with $\mathrm{V}$, Mo, W, or an oxynitride $\left(\mathrm{CrN}_{0.9} \mathrm{O}_{0.1}\right){ }^{151,152,177}$ This results, obtained even before a careful optimization of carrier concentration or nanostructuring to reduce thermal conductivity, shows that $\mathrm{CrN}$ is a promising material for thermo- electric applications. It rather cheap material compare to $\mathrm{ScN}$ and it can be synthesis as bulk, thin films or thick coating. In my study, I investigate the solid solution of $\mathrm{Cr}_{1-\mathrm{x}} \mathrm{Sc}_{\mathrm{x}} \mathrm{N}$ motivated by the fact that the $3 d$ orbitals of Sc is empty. Thus one can hope to exploit these empty $3 d$ orbitals to delocalize electrons in $\mathrm{Cr}$ $3 d$ orbitals, result in electrical resistivity reduction and possibly decreased thermal conductivity due to alloying element phonon scattering. The results of this study is shown in [Paper IV]. 



\section{Chapter 4}

\section{Theoretical calculations}

"The underlying physical laws necessary for the mathe-
matical theory of a large part of physics and the whole of
chemistry are thus completely known, and the difficulty
is only that the exact application of these laws leads to
equations much too complicated to be soluble. It there-
fore becomes desirable that approximate practical meth-
ods of applying quantum mechanics should be developed,
which can lead to an explanation of the main features of
complex atomic systems without too much computation."

Paul A.M. Dirac

in Containing Papers of a Mathematical and Physical Character, P. Roy. Soc. Lond. A Mat. Vol. 123, No. 792

In science, the theoretical descriptions of nature can be formed by analyzing the result of experiment, or by performing calculations based on existing theories and axioms. It is well acknowledged that theoretical modeling is a powerful tool for gaining information about the nature of materials in materials science. In this chapter, we will discuss the basic concept of the density functional theory (DFT), physical metallurgy and molecular dynamic (MD) formalism which have been used thought this Thesis. They are a theoretical scheme which is formulated to solve the complication of the quantum mechanics in first principle ( $a b$ initio) calculations for obtaining the phase stability, electronic and vibrational properties of materials.

\subsection{Ab-initio calculations}

In material science, our quest is to explain the nature of materials or engineer their properties. The materials properties are inherited by nature of the materials itself (type of elements, structures, chemical bonds). In principle, these chemical bonds, originating in the interaction between electrons and nuclei, can be obtained from quantum mechanics. One could imagine that by solving the 
Schrödinger equation, it would provide the microscopic properties that reflect all the relevant macroscopic properties of those materials. Thus this means we can predict the properties of new materials or suggest new path way to engineer our material for better properties.

This type of calculation is called "First principles" or "Ab initio" calculation, meaning the calculation from the beginning. ${ }^{178-182}$ However, we will end up with an intractable Schrödinger equation due to the number of particles (electron and nuclei) in the order of Avogadro's number coupled by the coulomb interaction of all charged particles which need to be solved. Obviously, the numerical computation with appropriate approximation will be the way to cope with this problem. Due to the continually refinement of the method and improvement of high performance computers, this allows the result of first principle calculations to become more accurate and valid in real material properties calculations. The success of this formalism brings to us a new era of material research moving away from experimental trial and error methodology to more precise theory driven approach in studying or engineering the materials.

\subsection{Density functional theory}

As we acknowledge quantum mechanics has a big success in describing atomic scale phenomena via the famous equation called Schrödinger equation ${ }^{\mathrm{I}}$ which can be written as

$$
i \hbar \frac{\partial \Psi\left(\mathbf{r}_{i}, \mathbf{R}_{I}, \boldsymbol{\sigma}_{i}, t\right)}{\partial t}=\hat{H} \Psi\left(\mathbf{r}_{i}, \mathbf{R}_{I}, \boldsymbol{\sigma}_{i}, t\right),
$$

The time independent Schrödinger equation is

$$
\hat{H} \Psi\left(\mathbf{r}_{i}, \mathbf{R}_{I}, \boldsymbol{\sigma}_{i}, t\right)=E \Psi\left(\mathbf{r}_{i}, \mathbf{R}_{I}, \boldsymbol{\sigma}_{i}, t\right),
$$

(I) The relativistic Dirac equation in one form or another is solved in practice. But to reduce mathematical complexity, the formalism of the Schrödinger equation is used here to describe the theory. where $\hbar$ is reduce Planck's constant $\left(\frac{h}{2 \pi}\right), \hat{H}$ is Hamiltonian and $\Psi\left(\mathbf{r}_{i}, \mathbf{R}_{I}, \boldsymbol{\sigma}_{i}, t\right)$ is wavefunction which is a function of position of electrons $\mathbf{r}_{i}$ and nuclei $\mathbf{R}_{I}$, spin of electrons and time in the system, and $E$ is the energy eigenvalue of each quantum states. As one could guess for such many-body systems, it is impossible to carry out this calculation in practice, since we have a huge number of coupled degrees of freedom that need to be solved to obtain ground state of electrons. Due to this complication, there are 
several approximations and models trying to simplify the problem. The Born-Oppenheimer approximation is a first simplification. ${ }^{183}$ This approximation comes from the fact that electrons have smaller mass than nuclei, yielding that electrons move faster than nuclei. Therefore when solving the electronic problems, we can neglect the change of nuclei position in the Hamiltonian, assuming them to be fixed in time, leaving only contribution from electrons in a fixed external potential from the nuclei. ${ }^{184}$ We can thus reformulate our electronic Hamiltonian in term of manybody interactions as

$$
\begin{aligned}
\hat{H}= & -\underbrace{\sum_{i} \frac{\hbar^{2}}{2 m_{e}} \nabla_{i}^{2}}_{\hat{T}}-\underbrace{\sum_{i, l} \frac{Z_{l} e^{2}}{\left|\mathbf{r}_{i}-\mathbf{R}_{l}\right|}}_{\hat{V}_{e x t}}+\underbrace{\frac{1}{2} \sum_{i \neq j} \frac{e^{2}}{\left|\mathbf{r}_{i}-\mathbf{r}_{j}\right|}}_{\hat{V}_{e e}} \\
& +\underbrace{\frac{1}{2} \sum_{l \neq k} \frac{Z_{l} Z_{k} e^{2}}{\left|\mathbf{R}_{l}-\mathbf{R}_{k}\right|}}_{\hat{V}_{I I}},
\end{aligned}
$$

where $\hbar$ is the reduced Planck's constant $\left(\frac{h}{2 \pi}\right), m_{e}$ is electron mass, $Z$ is atomic number. First term is the kinetic energy operator for the electrons $\hat{T}$, second term is the potential acting on the electrons from the nuclei $\left(\hat{V}_{\text {ext }}\right)$ which can be considered as external potential acting on electrons, third term is Coulomb interaction between electrons $\left(\hat{V}_{e e}\right)$ which is depended on separation between the position $\mathbf{r}$ and $\mathbf{r}^{\prime}$. Since the Coulomb interaction is long ranged, thus it will affect the electrons motion of other $N-1$ electrons which mean their motion is correlated, and the last term is the Coulomb interaction between nuclei $\left(\hat{V}_{I I}\right)$ which enter only as a constant in each considered geometry.

One of the effort on trying to solve this many-body problem by Hartree ${ }^{185-187}$ and later it was improved and applied to atoms by Fock. ${ }^{188}$ The Hatree-Fock method simplifies the many-body problem by transforming the problem into a set of self-consistent oneelectron model. This idea bases on minimizing the total energy with respect to all degrees of freedom in the total wave function of the system by using variational method to expectation value of Hamiltonian of the system, $\langle\Phi|H| \Phi\rangle$. So that the system wave function, $\Phi$ can be determined by a Slater determinant of single particle orbitals which will generate the coupled set of the nonlinear of all single particle wave function in the system, $\psi_{i}\left(r_{i}, \sigma_{i}\right)$ due to Pauli Exclusion Principle that does not allow electrons to 
stay on the same quantum states. Thus the system needs to be described as antisymmetric wave function. After the variational method we get Hatree-Fock equation which is shown as ${ }^{184}$

$$
\begin{aligned}
& {\left[\hat{T}+\hat{V}_{e x t(\mathbf{r})}\right] \psi_{i}\left(\mathbf{r}_{i}, \sigma\right)} \\
& +e^{2} \sum_{j, \sigma^{\prime}} \int \psi_{j}^{*}\left(\mathbf{r}_{j}, \sigma\right) \frac{1}{\left|\mathbf{r}-\mathbf{r}^{\prime}\right|} \psi_{j}\left(\mathbf{r}^{\prime}, \sigma^{\prime}\right) \psi_{i}(\mathbf{r}, \sigma) d \mathbf{r}^{\prime} \\
& -e^{2} \sum_{j, \sigma^{\prime}} \int \psi_{j}^{*}\left(\mathbf{r}^{\prime}, \sigma\right) \frac{1}{\left|\mathbf{r}-\mathbf{r}^{\prime}\right|} \psi_{j}(\mathbf{r}, \sigma) \psi_{i}\left(\mathbf{r}^{\prime}, \sigma^{\prime}\right) d \mathbf{r}^{\prime}=\varepsilon_{i} \psi_{i}(\mathbf{r}, \sigma),
\end{aligned}
$$

where the first terms groups together the single electron kinetic and external potential energy. The second term describes the repulsive Coulomb interaction occurs in pair of electrons so called Hartree term. Consequently when electrons are interchanged position and spin-orbital state, it will contribute another interaction to electrons in the system as the exchange interaction which is shown in the last term (Fock term) due to the antisymmetric quantum states. The solution of Hartree-Fock can obtain through the self-consistency method on the Hartree-Fock equation.

However, the Hartree-Fock approximation has not cover all degree of correlation interaction between electrons yield higher in total energy according to Hartree part. We will discuss on this issue on the next part. Given the complexity of the electrons wavefunction $\Phi$, it is wise to explain many-body electrons system in term of electron density $(n(\mathbf{r}, \sigma))$ or probability density of finding electrons at point $\mathbf{r}$ with spin $\sigma$. This reduces the many-coordinate problem to one parameter problem, i.e., we will work with only $3+1$ coordinates ( 3 from general coordinate and 1 is spin) instead of with $3 n$ coordinates (number three comes from three general coordinate, $n$ is a number of particles). ${ }^{178}$ By formalism of density, the Coulomb interaction energy can be decomposed into two parts ${ }^{184}$ which is direct and exchange interaction among the electrons as

$$
\begin{aligned}
\hat{V} & =\frac{1}{2} \int \frac{P(\mathbf{r}, \sigma ; \mathbf{r}, \sigma)}{\left|\mathbf{r}-\mathbf{r}^{\prime}\right|} d \mathbf{r} d \mathbf{r}^{\prime} \\
& =\frac{1}{2}\left[\int \frac{n(\mathbf{r}, \sigma) n\left(\mathbf{r}^{\prime}, \sigma^{\prime}\right)}{\left|\mathbf{r}-\mathbf{r}^{\prime}\right|} d \mathbf{r} d \mathbf{r}^{\prime}+\int \frac{\Delta n\left(\mathbf{r}, \sigma ; \mathbf{r}^{\prime}, \sigma^{\prime}\right)}{\left|\mathbf{r}-\mathbf{r}^{\prime}\right|} d \mathbf{r} d \mathbf{r}^{\prime}\right] \\
& =\hat{V}_{H}+\hat{V}_{x c}
\end{aligned}
$$

where $\hat{V}_{H}$ is the Hartree interaction energy and $\hat{V}_{x c}$ is the exchange-correlation interaction energy. $P(\mathbf{r}, \sigma ; \mathbf{r}, \sigma)$ is a pair-density 
distribution which can be described by the joint probability of finding one electron of spin $\sigma_{1}$ at point $\mathbf{r}_{1}$ and second electron of spin $\sigma^{\prime}$ at point $\mathbf{r}^{\prime}$, simultaneously. $n(\mathbf{r}, \sigma) n\left(\mathbf{r}^{\prime}, \sigma^{\prime}\right)$ is explained as the uncorrelated interaction of electrons i.e., the joint probability is just the product of individual probability of finding electron on each point and the degree of correlation, $\Delta n\left(\mathbf{r}, \sigma ; \mathbf{r}^{\prime}, \sigma^{\prime}\right)$ is the difference between correlated and uncorrelated electron density which is shown by

$$
\Delta n\left(\mathbf{r}, \sigma ; \mathbf{r}^{\prime}, \sigma^{\prime}\right)=P(\mathbf{r}, \sigma ; \mathbf{r}, \sigma)-n(\mathbf{r}, \sigma) n\left(\mathbf{r}^{\prime}, \sigma^{\prime}\right)=n_{x c}\left(\mathbf{r}, \sigma ; \mathbf{r}^{\prime}, \sigma^{\prime}\right)
$$

The Equation (4.6) can be interpreted as an electron at point $\mathbf{r}$, the exchange and correlation effects will repel electron giving a depletion region in the charge density around $\mathbf{r}$ which can be called as exchange-correlation hole with the charge density denoted as $n_{x c}$. This effect will reduce the probability of finding other electron around each electron producing a net weaker interaction among any two particles due to that region is screened from repulsive Coulomb potential from electrons as compare to a interaction of pure one-particle densities. This is also cancelled, at least partially self-interaction stemming from the independent particle ansatz. This is clearly shown that in Hartree-Fock approximation consider only the non-correlated interaction between densities of electrons and the exchange interaction due to Fock term.

By this interpretation, the effect of the exchange-correlation hole has included the effect of correlation interaction as the difference between the exact and uncorrelated interaction. This exchangecorrelation interaction is a back bone of density functional theory calculations, we will comes back to this topic later.

\subsection{Khon-Sham equation}

The Hartree-Fock scheme has a success to explain simple system like a few atoms or molecules, but the calculation still impracticable to solve for many-body systems like ionic/covalent crystals. Hohenberg and Kohn construct the core concept of modern density functional theory ${ }^{189}$ by using the idea of using electron density $(n(\mathbf{r}))$ instead of individual electrons to solve the complication of modern interacting many-body system. In addition Hohenberg and Kohn formulated two key theorems ${ }^{189}$ stated that 
Theorem 1 : For any system of interacting particles in an external potential $V_{\text {ext }}(\mathbf{r})$, the potential $V_{\text {ext }}(\mathbf{r})$ is determined uniquely, expect for a constant, by the ground state particle density $n_{0}(\mathbf{r})$.

This theorem results in the ground and excite states and all properties of such a system can be determined in principle for given only the ground state density $n_{0}(\mathbf{r})$.

Theorem 2: One can define a universal functional for the energy $E[n]$ in terms of the density $n(\mathbf{r})$ which is valid for any external potential $V_{\text {ext }}(\mathbf{r})$. For any particular $V_{\text {ext }}(\mathbf{r})$, the exact ground state energy of the system is the global minimum value of this functional, and the density $n(\mathbf{r})$ that minimizes the functional is the exact ground state density $n_{0}(\mathbf{r})$.

This theorem demonstrates how the ground state density can be determined by a minimization procedure of energy functional for any particular $V_{\text {ext }}(\mathbf{r})$.

Based on these two theorems Kohn and Sham formulated the key equations that is a back bone of DFT, today. ${ }^{190}$ They are based on replacing the real electrons with non-interacting electron with the same density as the real system of interacting electrons and all the interaction terms are inserted in a form of an effective potential. The Kohn-Sham energy functional ${ }^{\mathrm{II}}$ can be written as

$$
E[n]=T_{s}[n]+\int d \mathbf{r} V_{e x t}(\mathbf{r}) n(\mathbf{r})+E_{H}[n]+E_{x c}[n]+E_{I I},
$$

where $T_{s}[n]$ is the kinetic energy functional of the non-interacting particles,

$$
T_{s}[n]=\frac{1}{2} \sum_{i=1}^{N}\left\langle\varphi_{i}\left|\nabla^{2}\right| \varphi_{i}\right\rangle,
$$

$E_{H}[n]$ is the Hartree energy functional of the density

$$
E_{H}[n]=\frac{1}{2} \int \frac{n(\mathbf{r}) n\left(\mathbf{r}^{\prime}\right)}{\left|\mathbf{r}-\mathbf{r}^{\prime}\right|} d \mathbf{r} d \mathbf{r}^{\prime},
$$

$E_{x c}[n]$ is the exchange-correlation energy density functional for

(II) Here we express all expression in Hartree atomic unit. $\left(m_{e}=h=e=1\right)$ which the exact form is not known. But it can be approximated as

$$
E_{x c}[n]=\int n(\mathbf{r}) \epsilon_{x c}([n], \mathbf{r}) d \mathbf{r},
$$


where $\epsilon_{x c}([n], \mathbf{r})$ is the exchange-correlation energy density which is expressed as

$$
\epsilon_{x c}([n], \mathbf{r})=\frac{1}{2} \int \frac{\bar{n}_{x c}\left(\mathbf{r}^{\prime}\right)}{\left|\mathbf{r}-\mathbf{r}^{\prime}\right|} d \mathbf{r}^{\prime}
$$

and $\bar{n}_{x c}$ the coupling-constant-averaged hole can be expressed as

$$
\bar{n}_{x c}=\int_{0}^{1} n_{x c}^{\lambda}\left(\mathbf{r}, \mathbf{r}^{\prime}\right) d \lambda,
$$

where $n_{x c}^{\lambda}\left(\mathbf{r}, \mathbf{r}^{\prime}\right)$ is exchange-correlation density which was defined in Equation (4.6), $\lambda$ is the coupling constant which is described how correlate of electrons, this integration can be understood in terms of the potential energy which is considered from $\lambda=0$ means non-interacting electrons system, only exchange interaction is taken into account and $\lambda=1$ is all electrons are fully interacting or correlated. This connection is determined from adiabatic connection formulation. ${ }^{184,191}$ Since the exchangecorrelation hole density obeys the sum rule thus the couplingconstant-averaged hole should equal to $1,{ }^{184,192}$ so the correlation hole must integrate to zero leading to redistribution of the density of holes (electrons density depletion region). ${ }^{184}$ The last term $E_{I I}$ is the energy from interaction between nuclei. A variational process to minimize the total energy functional leads to a Kohn-Sham equation that is

$$
\left(-\frac{1}{2} \nabla^{2}+V_{e f f}(\mathbf{r})\right) \varphi_{i}=\varepsilon_{i} \varphi_{i},
$$

where $\varphi_{i}$ is single-particle wavefunction, $\varepsilon_{i}$ is energy that corresponding to that state, and $V_{s}(\mathbf{r})$ is the effective potential which is given by

$$
V_{e f f}(\mathbf{r})=V_{e x t}+\frac{\delta E_{H}[n(\mathbf{r})]}{\delta n(\mathbf{r})}+\frac{\delta E_{x c}[n(\mathbf{r})]}{\delta n(\mathbf{r})} .
$$

These Khon-Sham equations are a very useful approximation when attempts of first-principles calculations of the properties of condensed matter are done. However, the Khon-Sham equation cannot be solved exactly unless the exchange-correlation energy functional of the density has been defined exactly to correct the spurious, self-interaction energy functional from Hartree energy functional of the density, and other explicit many body effects. Unfortunately, there is no established way for obtaining the exchange-correlation holes density exactly, thereby the exchangecorrelation energy functional of the density need to be approximate. Hence in the next Section, we will discuss about exchangecorrelation approximations. 


\subsection{Exchange and correlation functional approximations}

Khon-Sham equations are obtained by replacing all electrons in the system with non-interacting electrons with the same electron density. To recover the many-body phenomena that missing in such a single-particle scheme, the exchange-correlation term is introduced which contains all the missing many particle effects, deriving the exchange-correlation is nontrivial. Fortunately, the exchange-correlation energy can be approximated because it is relatively small when compare with the kinetic energy and Hartree energy which can be exactly determined. Here in this Thesis four common functional approximations have been discussed which are the Local Density Approximation (LDA), ${ }^{190}$ Generalized Gradient Approximation (GGA), ${ }^{193,194}$ Hubbard-corrected ("+U") approximation (LDA+U or GGA+U), ${ }^{195-197}$ and Hybrid functionals. ${ }^{198-200}$

\subsubsection{Local density approximation}

The LDA ${ }^{\mathrm{III}}$ has been proposed together with the DFT calculation by Khon and Sham. ${ }^{190}$ In LDA, the exchange-correlation energy can be obtained easily by an integral over all space of the production of density with the exchange-correlation energy per particle of a homogenous electron gas with its density,

$$
E_{x c}^{\mathrm{LDA}}[n]=\int n(\mathbf{r}) \epsilon_{x c}^{\mathrm{hom}}([n(\mathbf{r})]) d \mathbf{r} .
$$

By using quantum Monte Carlo simulation, the exchangecorrelation energy of the homogenous electron gas interacting with a homogenous positive potential background has been obtained. ${ }^{201}$ The LDA has yielded great successes in term of determining the properties of materials. The background is that the exchange-correlation hole of all electronic system obeys the constraints imposed by the sum rules. This means we can obtain quite accurate exchange-correlation hole density from any physi-

(III) The local spin density approximation (LSDA) is more general approximation including magnetic polarization effects. cal Hamiltonian, like the LDA, even if it is not the correct Hamiltonian.

In addition, the Equation (4.15) shows that it is the system and spherically average of the exchange-correlation hole that enters 
to the exchangecorrelation energy functional meaning that the detailed shape of the exchange-correlation hole does not need to be correct. On the other hand, LDA has also weaknesses when it comes to considering real systems, where the electron density does not have homogenous distribution as the approximation assumes. ${ }^{192,202}$ This error leads to overbinding due to overlap of atomic exponential tail in the bonding. Because in real matter, the electron density has a big variation when it is considered far away from point $\mathbf{r}$ in space as the positive potential is not homogenous but located at the nuclei in difference point in space which make electron density varying in space. This is highlighted in systems with narrow bands, such as system consisting of $3 d, 4 f$ or $5 f$ valence electrons. ${ }^{174,196,203}$ To improve this weakness of LDA, we need to develop another approximation that allows consideration of the change of electron density.

\subsubsection{Generalized gradient approximations}

As it is stated in the name of the approximation, the exchangecorrelation energy functional can be expanded in terms of the density and its density gradients as

$$
E_{x c}^{\mathrm{GGA}}[n]=\int n\left(\mathbf{r} \epsilon_{x c}^{\mathrm{hom}}([n]) f(\nabla n, n, \ldots) d \mathbf{r}\right) .
$$

At first of the development of this approximation, it did not give consistent improvement over LDA because the gradient expansion of electron density disobeys the sum rules and other relevant conditions giving worse results because inaccurate of exchangecorrelation hole density. So the term "generalized" denotes a variety of ways proposed to modify the expansion (gradient) in term of a function, $f(\nabla n, n, \ldots)$ that is selected to fulfill the constrains to give an accurate results of the calculations. Those methods likes B88-GGA, ${ }^{194}$ PW91-GGA, ${ }^{204}$ and PBE-GGA, ${ }^{205}$ are the most commonly used to calculate the electronic structure of condensed matter nowadays. From this approximation the lattice parameter and of course other properties relate to volume of unit cell of most transition metals systems were calculated more precise compare to LDA. Because of the soft electron density in GGAs leads to exchange energy lower than the LDA. This results in correction of overbinding in the LDA. For correlation energy, it is expressed as LDA with an additive term that depends on the gradients which 
(IV) In general, the energy eigenvalues that obtain from Khon-Sham equation have no direct physical meaning. The electronic band structure which is constructed from ground and excitation of both eigenvalues and eigenfunctions, thereby, is not correct structure, even if the exact exchange-correlation energy functional is used. There are many approaches used to give a well-defined meaning in order to construct the electronic band structure such as quantum Monte Carlo or many-body perturbation methods by using total energy functional and Kohn-Sham solutions. Nevertheless, LDA and GGAs Kohn-Sham band structure has been shown to be a good approximation to experimentally obtained valence band spectra, except for the values of the band gaps. ${ }^{184}$ And so called strongly correlated material. show decreasing of correlation energy and disappeared at large density gradient. However the GGA instead give a slightly overestimated lattice parameter in some case. ${ }^{206,207}$

In addition, there are many reports show that both LDA and GGA underestimate the band gap of well-known semiconductor as well as transition metal nitride. ${ }^{127,128,174,208,209}$ It is well known that LDA fails to represent the electronic band structure of semiconductor materials in which cannot correct by using GGAs since they developed from the LDA. There are several reasons for this. First, the energy eigenvalues that are calculated from selfconsistency of Khon-Sham equation by using any functional do not represent exact energy eigenvalues ${ }^{\mathrm{IV}}$ Besides, the energy band gaps from Kohn-Sham equation do also not represent to true energy gaps due to a contribution from the discontinuity of derivatives of the exchange-correlation functionals are missing which LDA and GGAs do not take this effect into account (see ref. [210] chap. 2). Second, LDA and GGA have a common error on incompletely compensating the self-interaction energy in the Hartree interaction. For example in the strong correlation system which electrons are localized, thus the electron correlation are screened making the strong chemical bonds instead of weak bonds in reality. One attempt to solve this issue is by introducing the Hubbard $(+\mathrm{U})$ term which add the effect of a screened coulomb potential to the strong correlated orbital. We will discuss this approximation in the next Section.

\subsubsection{Hubbard-corrected (“+U”) approximation $(\mathrm{DFT}+\mathrm{U})$}

In the strong correlation system, there is a mixture in electrons contribution i.e., the large degree of localization in $d$ or $f$ orbitals and the small degree of localization (delocalization) in $s$ and $p$ orbitals. These contributions give LDA and GGA fail predicting some physical phenomena such as transition-metal oxide properties or Mott insulator. ${ }^{196,203,211}$ This is due to LDA and GGA functionals are over delocalized electrons in $d$ and $f$ orbitals, since the electrons can feel their own potential from incompletely canceling the self-interaction. One idea to solve this problem is introducing the Hubbard-corrected term which adds the Coulomb interaction between electrons into the localized orbital while we treat all elec- 
trons in the system with LDA or GGA. From this idea, we can write the Hubbard-corrected exchange-correlation Hamiltonian ${ }^{\mathrm{V}}$ as ${ }^{195,212,213}$

$$
\hat{H}^{\mathrm{LDA}+\mathrm{U}}=\hat{H}^{\mathrm{LDA}}+\hat{V}^{\mathrm{U}} .
$$

Here the $\hat{V}^{\mathrm{U}}$ includes a double counting (DC) term. Since the LDA Hamiltonian already has the contribution from the orbital related energy, therefore we need to remove the contribution from the Hubbard-corrected term so that we are not count twice of their contributions. Since, the orbitals in LDA are non-linearly mixed, thus the exact DC term cannot be expressed in the orbitals basis. ${ }^{214}$ Nevertheless it can be approximated by "around meanfield" (AMF) or "fully localized limit" (FLL) of the Hubbard term in the DFT spirit. ${ }^{197}$ This $\hat{V}^{\mathrm{U}}$ is based on the Hubbard model for the Coulomb interaction between two electrons in the localized orbitals that are described in the orbital and spin basis $|m \sigma\rangle$ inside subset of orbital $\mathcal{O}$ as suggested by Anisimov et al. ${ }^{195,212}$

$$
\hat{V}^{\mathrm{U}}=\frac{1}{2} \sum_{\sigma_{1} \sigma_{2}} \sum_{m_{1}} U_{m_{2} m_{3} m_{4}} U_{m_{1} m_{2} m_{3} m_{4}} a_{m_{3} \sigma_{1}}^{\dagger} a_{m_{4} \sigma_{2}}^{\dagger} a_{m_{2} \sigma_{2}} a_{m_{1} \sigma_{1}},
$$

where $U_{m_{1} m_{2} m_{3} m_{4}}$ is the matrix elements of screened Coulomb interaction which $U_{m_{1} m_{2} m_{3} m_{4}}=\left\langle m_{3} m_{4}|| \mathbf{r}-\left.\mathbf{r}^{\prime}\right|^{-1} \mid m_{1} m_{2}\right\rangle, m_{i}$ is the orbital angular momentum of electron at site $i$ which is related to angular momentum of electron at site $i m_{i}=-l_{i}, \ldots, l_{i}$ and $\sigma_{i}$ is the spin states of electrons at site $i$. From the Equation (4.17) and (4.18) then using rotationally invariant suggested by Dudarev et al. ${ }^{215}$ we can write a new functional for exchange-correlation energy as :

$$
\begin{aligned}
E^{\mathrm{LDA}+\mathrm{U}}[n] & =E^{\mathrm{LDA}}[n]+E^{\mathrm{U}}\left[o_{i}^{\sigma}\right] \\
& =E^{\mathrm{LDA}}[n]+\frac{U-J}{2} \sum_{\sigma}\left(\sum_{i} \rho_{i j}^{\sigma}-\sum_{i j} \rho_{i j}^{\sigma} \rho_{i j}^{\sigma}\right) .
\end{aligned}
$$

where $\rho_{i j}^{\sigma}$ is a density matrix on site $i$,

$$
\begin{aligned}
& \rho_{i j}^{\sigma}=o_{i}^{\sigma} o_{j}^{\sigma} \\
& o_{i}^{\sigma}=\int n^{\sigma}(r) \psi_{i}(r) d r
\end{aligned}
$$

where $o_{i}$ is the occupation of states projected onto the electron density at site $i$ using the atomic orbitals $\psi_{i}$. Then summation over $i$ and defines $U-J$ as the effective $U$ ( $\left.U^{\text {eff }}\right)$, we get total
(V) In principle this Hubbardcorrected approximation can be applied to both LDA and GGA. 
energy

$$
E^{\mathrm{LDA}+\mathrm{U}}[n, o]=E^{\mathrm{LDA}}[n]+\frac{U^{\mathrm{eff}}}{2} \sum_{i \neq j} o_{i} o_{j}-\frac{U^{\mathrm{eff}} N(N-1)}{2},
$$

where $N=\sum_{i \neq j} o_{i} o_{j}$ and designates $U^{\text {eff }}$ as $U$ from now on. This is the reason for the name of this functional LDA $+\mathrm{U}$. From the Equation (4.20), the last term, $\left[\frac{U^{\mathrm{eff}} N(N-1)}{2}\right]$ is come out from the around mean field approximated double counted term. ${ }^{197}$ Then taking derivative of Equation (4.20) with respect to the occupation number of orbital $o_{i}$ to obtain the orbital eigenvalue of $o_{i}$, we get

$$
\begin{aligned}
\epsilon_{i}^{\mathrm{LDA}+\mathrm{U}}=\frac{\partial E^{\mathrm{LDA}+\mathrm{U}}}{\partial o_{i}} & =\epsilon_{i}^{\mathrm{LDA}}+U \sum_{i \neq j} o_{i}-U N+\frac{U}{2} \\
& =\epsilon_{i}^{\mathrm{LDA}}+U\left(N-o_{i}\right)-U N+\frac{U}{2} \\
& =\epsilon_{i}^{\mathrm{LDA}}+U\left(\frac{1}{2}-o_{i}\right) .
\end{aligned}
$$

From the Equation (4.21), we can qualitatively interpret the physical effect of the introduction of the Hubbard term. Let consider the case of fully occupied and empty orbitals, the Hubbard term will shift the LDA energy of the fully occupied down by $-U / 2$, while the LDA energy of fully empty orbitals will raise by $U / 2$. This results in the electronic states become more localized in space and energy, as compared to the pure LDA solutions. Thus, the $\mathrm{LDA}+\mathrm{U}$ is primarily a way to compensate for the overdelocalization of LDA. These corrections may lead to the correct band gap solution, since the occupied bands are lower and empty bands are raised resulting in the energy gap appears in the electronic states. Moreover, this also correct some of self-interaction error from LDA or GGA which lead to more accurate physical prediction. However, this Hubbard-corrected approximation is very system dependent not only effect on the strong electrons correlated system, but the $U$ itself also system dependent which it need to test the value of $U$ against the experimental information to determine the appropriate $U$ for the further calculation. Thus one can consider this approximation is less $a b$ initio compare to another approximation or method. Nevertheless, the Hubbardcorrected approximation consumes less computer resource compare to more advanced approximation or method and give quite accurate results after obtaining a correct $U$. This is why Hubbardcorrected approximation still popular nowadays see ref. [213] for 
the further discussion on current DFT+U. So far we have introduce the first idea on how to solve the self-interact error in LDA and GGA. If we consider, Hartree-Fock approximation that shows the self-interaction is completely canceled by the exchanged interaction term as we discussed in the Section 4.2. However, the correlation energy is not present in Hartree-Fock approximation. Extra degrees of freedom can be introduced in the wavefunction in order to include the correlation interactions. However, this results in an increasing of computational cost with Hartree-Fock already requiring high computational resources due to wavefunction calculations. Thus, the hybrid functionals method has been introduced to solve this problem which we will discuss in next Section.

\subsubsection{Hybrid functionals}

The failures of LDA and GGAs exchange-correlation energy density functional on reproduce electronic band structure of most of semiconductors and lack of correlation interaction in HartreeFock approximation. However, both LDA and GGAs and HartreeFock approximation compensate their disadvantage by another advantage. This leads Becke takes advantage on the fact that the average exchange-correlation hole density depends on the coupling constant, which indicates the interaction of the electrons system leading him to approximate the exchange-correlation energy as linear combination of Hartree-Fock exchange and LDA or GGAs exchange and correlation energy. ${ }^{198,199}$ The attempt of Becke to replicate the intermediate regime of exchange-correlation hole density and the development of PBE-GGAs by Perdew et al. ${ }^{216-218}$ leads them to develop hybrid functional that correspond to the exchange-correlation energy in a form of

$$
E_{x c}^{\mathrm{PBE} 0}=E_{x c}^{\mathrm{PBE}-\mathrm{GGA}}+a\left(E_{x}^{\mathrm{HF}}-E_{x}^{\mathrm{PBE}-\mathrm{GGA}}\right),
$$

where $a$ is adjustable coefficients provided by fitting of experimental data, Perdew suggested $a=1 / 4$ that is determined by perturbation theory showing the most accuracy to experimental result. ${ }^{218}$ Since, the repulsive Coulomb interaction has two parts that is direct interaction which is long ranged and exchange interaction that takes care of the self-interaction is more short ranged. Heyd et al. ${ }^{200}$ takes this fact and separates the Coulomb potential into short-range and long-range component by using error 
function as a function of product between adjustable parameter governing the short-range interaction, $\omega$ and range, $r=\left|\mathbf{r}-\mathbf{r}^{\prime}\right|$. This leads to an exchange-correlation energy written as

$$
\begin{aligned}
E_{x c}^{\mathrm{HSE} 03}= & a E_{x}^{\mathrm{HF}, \mathrm{SR}}(\omega)+(1-a) a\left(E_{x}^{\mathrm{PBE}-\mathrm{GGA}, \mathrm{SR}}(\omega)\right. \\
& \left.+E_{x}^{\mathrm{PBE}-\mathrm{GGA}, \mathrm{LR}}(\omega)+E_{c}^{\mathrm{PBE}-\mathrm{GGA}}\right),
\end{aligned}
$$

which is called HSE03 hybrid functionals, ${ }^{200}$ where a is again adjustable coefficients similar to Perdew et al. ${ }^{218}$ suggested. From this equation see that Heyd focus on modifying the exchange energy since it can be called the one source that hinder the DFT calculation as Becke stressed this point. However, the DFT calculation bases on HSE03 hybrid functionals due to slowly converges causing high demanding of computational time. Therefore, HSE06 hybrid functional has developed to solved this problem ${ }^{219}$ by downsampling of short range Hartree-Fock exchange energy in reciprocal space yielding the results of this hybrid functional somewhat similar to PBE0 since downsampling lead to independent of $\omega$, but converges faster than PBE0 with respect to the number of k-point. So far, the results from DFT calculation by using hybrid functionals scheme show the most accurate due to the different portion of correlation energy and exchange energy are mixed in order to obtain exchange-correlation energy without the self-interaction energy. These results lead to significant improvement of $a b$ initio calculations of the most semiconductor and insulator materials. ${ }^{209,220-222}$

\subsubsection{Pseudopotentials and Projector augmented wave}

In order to apply the Kohn-Sham equations to real material systems, we need consider all the atoms in the crystal in our calculations. Luckily, we can simplify this problem by using Bloch's theorem due to the periodicity of the crystal lattice. In the Bloch's theorem shows that, the wavefunctions of the electron in the crystal can be considered as plane waves modulated with a function with the periodicity of the lattice. ${ }^{36,37}$ Plane waves are thus a convenient basis set to expand the wavefunction in the computation. Unfortunately, electrons wavefunction will oscillate rapidly when they close to the nuclei (localized behavior) and vary slowly at valence region (delocalized behavior) meaning that many plane 
waves are needed in order to calculate both electrons at core region than valence region. This results in a high requirement of computation resources and time. The pseudopotentials concept is introduced to deal with this issue (see ref. [184] chap. 11 for more details). This pseudopotential concept for condensed matter calculation was developed from the scattering problem that considers the effect of core electron scattering and behavior of valence wavefunction via the partial wave concept. A set of plane waves is used to describe the electron in the system which can be defined as modified valence wavefunctions. They consist of linear combination between true core and valence wavefunctions. There are modified valence wavefunction contains the sum over core states, thus the energy eigenvalue similar to the unmodified wavefunctions. However the transition from core region to valence region does not lead to a smooth pseudopotential. Therefore the projector augmented wave (PAW) method ${ }^{223}$ is implemented to deal with this problem which has been used extensively in this Thesis. In PAW, the concept of reducing number of plane waves by projecting the wavefunctions of the core electrons onto smooth auxiliary wavefunction via the transformation operator. We use the frozen-core approximation which assumes the core electrons do not mainly participate in chemical bonding. The core electrons smooth auxiliary wavefunctions are determined by original wavefunction outside the core region. Then the properties of core state will be determined only once with assistance of the smooth wavefunctions. However for the total energy evaluation, the full reconstructed wavefunction has been used to increase reliability. The size of the plane wave basis set is determined by a cut off for their energies: $\varepsilon(\mathbf{k})=\frac{1}{2} \mathbf{k}$, as

$$
|\mathbf{K}+\mathbf{k}|<K_{\text {cut }}
$$

where $\mathbf{k}$ denotes the sampled points of reciprocal space and $\mathbf{K}$ is a reciprocal lattice vector. Calculated properties need to be converged with respect to the value of $K_{\text {cut }}$ 

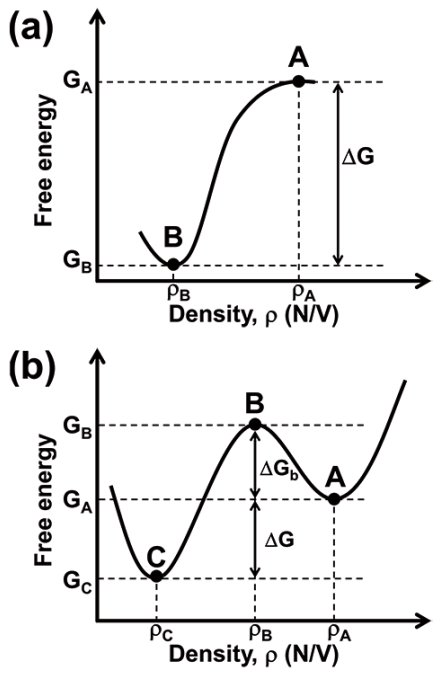

FIGURE 4.1: The schematic plot of Gibbs free energy versus material density. (a) Only one global minimum of Gibbs free energy (Point B), and (b) There are both local minimum (Point A) and global minimum of Gibbs free energy (Point B) which is separated by the Gibbs free energy barrier $\Delta G_{b}$

(VI) According to IUPAC, the adjective "free" is banned, thus they should be Gibbs energy and Helmholtz energy. 225

\subsection{Phase stability}

As we discussed in Chapter 3, the suggested strategies for improving thermoelectric figure of merit $\left(Z T_{m}\right)$ present-day involve doping, making solid solution, forming nano-inclusion, or stack them as superlattice. According to the applications of thermoelectrics, materials need to sustain a large temperature gradient. Therefore, this temperature gradient can possible change the phase of materials due to the thermal activated bulk diffusion causing phase separation in solid solution, mixing of nano-inclusion, or the diffusive interface in superlattice. These changes will positively or negatively effect thermoelectric properties of the materials. Therefore the knowledge of phase stability of thermoelectric materials is vitally important to determine in order to find a suitable strategy for improving their thermoelectric properties, accordingly. Here we used DFT calculations to help us predict phase stability trend of materials.

In order to determine thermodynamic phase stability of the materials, we have to consider their free energy. ${ }^{32,224}$ There are two free energy, we can consider i.e., Gibbs free energy $G$ and Helmholtz free energy $F .^{\mathrm{VI}}$ In this Thesis we consider the Gibbs free energy which is defined for the system at constant temperature $T$ and pressure $P$,

$$
G=H-T S,
$$

where $S$ is the entropy, and $H$ is the enthalpy, which is given by

$$
H=E+P V,
$$

where $E$ is the internal energy and $V$ is the volume. For Helmholtz free energy is defined for the system at constant temperature $T$ and volume $V$,

$$
F=E-T S \text {. }
$$

Therefore, if we say a material is in a thermodynamically stable phase or state, we always refer to a phase or state of the material that has thermodynamic equilibrium in a global minimum of free energy. ${ }^{226,227}$ When Gibbs free energy is a global minimum meaning that the system or material is thermodynamic equilibrium under constant $T$ and $P$. Whereas Helmholtz free energy is a global minimum meaning that the system or material is thermodynamic equilibrium under constant $T$ and $V$. 
The material that stay at this phase or state will resist to the perturbations. On the other hand, a material in a thermodynamically unstable phase or state, we describe as a phase or state of the material that has conditions where very small fluctuations to the material lead to spontaneous phase or state transformation to the stable phase due to a thermodynamic driving force, ${ }^{226,227}$ $\Delta G=G_{2}-G_{1}$ (see Figure 4.1(a)).

Next let us consider the Figure 4.1(b), there are two Gibbs free energy minimums i.e., local minimum at Point $\mathrm{A}$ and global minimum at point $C$. In this case, the phase at point $C$ is more stable than point A. Since, there is thermodynamic driving force which is equal to $\Delta G$ acts on the phase at point A. Ideally, if the free energy barrier is equal to zero $\Delta G_{b}=0$, the driving force $\Delta G$ will drive the material to Point $\mathrm{C}$ which is more energetic favorable thought phase transformation. Because the existence of the driving force the phase or state at Point $\mathrm{A}$ is an thermodynamic unstable $\left(\Delta G_{A}>\Delta G_{C}\right)$. But, the existence of the $\Delta G_{b}$ prevents the material from phase transformation unless the material can overcome the $\Delta G_{b}$. One can consider that $\Delta G_{b}$ is the energy requires to activate the atomic diffusion for atoms in the system or to generate a new surface (interface energy). So that they can diffuse to new equilibrium position with the driving force $\Delta G$. This means that the phase transformation is no longer spontaneous process. The rate of phase transformation is proportional to $\exp \left(G_{b} / k_{\mathrm{B}} T\right)$, where $k_{\mathrm{B}}$ is the Boltzmann's constant and $T$ is the absolute temperature. Alternately, we can say that our material is kinetically trapped in local minimum of free energy. Since the rate of phase transformation depends on the activation energy $\left(G_{\text {act }}=G_{b}\right)^{\mathrm{VII}}$. Here the phase or state at point A can be defined as thermodynamically metastable $\mathrm{e}^{224,226,227}$ :

Thermodynamically metastable is a phase or state of the material that resides at a local minimum in free energy not a global one meaning that it is thermodynamic unstable. The presence of the free energy barrier between metastable and globally stable phase or state makes the system can stand some external variations like thermal fluctuations. Before the thermodynamic driving force transforms the system to the more energetic stable phase or state within the rigorous limit of infinite time.
(VII) Note that all the energy can be converted to a thermal energy $\left(k_{\mathrm{B}} T\right)$ 


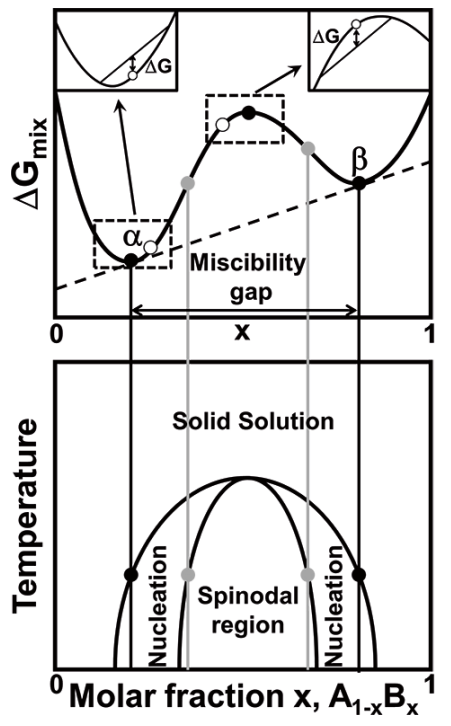

FIGURE 4.2: The schematic plot of (a) the mixing Gibbs free energy with the molar fraction of solid solution $A_{1-x} B_{x}$. The plot shows that this system has a miscibility gap where the solid solution with composition in the gap will reduce its Gibbs free energy by decomposition into $\alpha$ and $\beta$ phase, and (b) the related phase diagram indicates nucleation and growth or spinodal region.
The common examples for the metastable system are the phase in ambient pressure carbon i.e., diamond (metastable) to graphite (stable), superheated water (liquid) where the pure water in the clean container can be heated above boiling point without boiling (phase transition from liquid to gas phase), supercooled water (liquid) where the pure water can be cooled below freezing point without freezing, or supersaturation solutions where the large amount of solute that exceeds the equilibrium solubility can be dissolved in the solvent. All these phase transition process will eventually occur in a period or time. But we can increase the rate of phase transformation by adding some extra energy (increase kinetic energy) or provide nucleation sites, impurities (reduce free energy barrier). The metastable material is quite interesting in itself since they can possess the properties of both stable and unstable phase and this will yield the great deal on solid solution materials.

\subsubsection{Phase stability of solid solution and decomposition processes}

So far we have discussed only the single component phase stability. Next we consider the phase stability of two components (binary) system. ${ }^{224,226,227}$ Here we make a fictive substitutional solid solution $A_{x_{A}} B_{x_{B}}$ of $N$ atoms consists of $A$ atoms with molar fraction of $x_{A}$ and $B$ atoms with molar fraction of $x_{B}$, where $x_{A}+x_{B}=1$. We can define the Gibbs free energy of this solid solution as

$$
G=G_{A} x_{A}+G_{B} x_{B}+\Delta G_{m i x}\left(x_{A}, x_{B}\right)
$$

where, $G_{A}$ is the Gibbs free energy of pure $A, G_{B}$ is the Gibbs free energy of pure $B, \Delta G_{m i x}$ is defined as

$$
\Delta G_{m i x}\left(x_{A}, x_{B}\right)=\Delta H_{m i x}\left(x_{A}, x_{B}\right)-T \Delta S_{m i x}\left(x_{A}, x_{B}\right),
$$

where $\Delta H_{m i x}$ is the mixing enthalpy of an solid solution and $\Delta S_{\text {mix }}$ is the mixing entropy of an solid solution. Generally, the $\Delta G_{m i x}(x)<0$ the system will prefer to form solid solution and $\Delta G_{m i x}(x)>0$ the system will prefer phase separation. The schematic Gibbs free energy of our fictive solid solution with respect to their molar fraction at given temperature $T$ is shown in Figure 4.2(a). It appears that there are two minima i.e., there 
are two stable phases which correspond to $A$ rich $\alpha$ phase and $B$ rich $\beta$ phase. Thus we can draw a common tangent line connect between these two minima. This common tangent is represented the equilibrium between these two phases. For any composition between the composition of these two phase, the lowest $\Delta G_{m i x}$ can be now represented by this common tangent line. And the equilibrium state will be a mix of two different phases.

Thus the miscibility gap is appeared. Since any homogenous solid solution with overall composition of $x$ that has $G_{m i x}(x)$ above this common tangent line will be unstable phase. Due to there is a driving force $(\Delta G)$ will force this solid solution to decompose into $\alpha$ phase and $\beta$ phase so that the system can reduce its energy to equilibrium. The proportion between these two phase after decompose will be determined by the overall alloy composition before its decompose, and can be determined by the lever rule $\mathrm{V}^{\mathrm{VIII}}$.

There are two possible mechanisms for the phase decomposition which is separated by the grey point in the Figure 4.2(a). This point represents a locus of inflection point of the mixing Gibbs free energy curve where the second derivative of mixing Gibbs free energy with respect to concentration (curvature) is zero, $d^{2} G_{m i x} / d x^{2}=0$. Thus these two phase decomposition mechanisms are represented as below the point the grey point where $d^{2} G / d x^{2}>0$ which we call this region as the nucleation and growth decomposition region and above the grey point where $d^{2} G / d x^{2}<0$ which we call this region as spinodal decomposition region (shown in Figure 4.2(b)). ${ }^{224,227}$

First let consider the region where $d^{2} G / d x^{2}>0$, The small fluctuation in concentration leads to an increase in Gibbs free energy (see a left inset in Figure 4.2(a)). This means that the decomposition process is a kinetics limited. Since, this decomposition process requires the size of nucleus (cluster of alloyed atoms) is large enough to overcome the interface related nucleation barrier (relate to Gibbs free energy barrier) so that the new phases can be grown. This interface related nucleation barrier is the energy that require to create new surface, and the elastic strain energy that occurs due to an paternally coherent interface between new phases and parent phase causing the new phases strained; if the new phases and parent phase have incoherent interface, thus there is no elastic strain energy. Hence, the decomposition mechanism in
(VIII) In case of weak positive mixing enthalpy, the miscibility gap can be disappeared, since $\Delta S_{\text {mix }}$ is always positive which at high enough temperature could dominate $\Delta \mathrm{G}_{\text {mix }}$ through $-\mathrm{T} \Delta \mathrm{S}_{\text {mix }}$. So that we will get stable solid solution phase as shown in top region of phase diagram (Figure 4.2(b)) 
this region is called nucleation and growth decomposition. ${ }^{224,227}$ Because of this interface related nucleation barrier, we can consider that the alloy within this composition region is in metastable phase in thermodynamic sense.

On the other hand, the region that has $d^{2} G / d x^{2}<0$, in this case a small concentration fluctuation will lead to a decrease in Gibbs free energy (see a right inset in Figure 4.2(a)). Therefore this decomposition mechanism does not require to overcome free energy barrier; the decrease in Gibbs free energy will cause the spontaneous decomposition. We can call this decomposition mechanism Spinodal decomposition. ${ }^{224,227}$ Therefore, the alloy with this composition region can be considered as thermodynamically unstable.

Note that we can defined the point where $d^{2} G_{m i x} / d x^{2}=0$ as a spinodal where a locus of state or phase that represents the limit of possible metastability in thermodynamic sense and the binodal is the borderline of phase separation and stable solid solution. This is shown in Figure 4.2(b). Although an alloy inside the spinodal is thermodynamically unstable; this does not mean that we cannot synthesis such an alloy. A technique like magnetron sputtering (will be discussed in next Chapter) allows us to synthesize a material far from thermodynamic equilibrium. If the synthesis temperature is low enough, bulk diffusion cannot occur prohibiting phase separation. As diffusion is required, there is not enough for the solid solution to decompose; this means that the solid solution is unstable in thermodynamic sense, but still metastable and possible to synthesize due to kinetic limitations. The famous example for such a case is the $\mathrm{Ti}_{1-\mathrm{x}} \mathrm{Al}_{\mathrm{x}} \mathrm{N}$ alloy system. ${ }^{207,228}$

\subsection{Phase Stability Calculations}

As we discussed at the beginning of previous section, the possibility of phase stability prediction will enable us to select or design a suitable $Z T_{m}$ improvement strategy for our materials system. Computer calculations within the DFT scheme allow us to predict the phase stability by calculating the $\Delta G$ Furthermore this will help us save a lot of resource and time involve in thermoelectric materials development. In this Thesis, we theoretically study the phase stability of nitride solid solution materials and whether they will form disordered solid solution, ordered solid solution or 
phase separation. For example let us consider the phase stability of our fictive $A_{1-x} B_{x}$ solid solution. To determine its phase stability, we need to calculated the $\Delta G_{m i x}$ of this substitutional solid solution ${ }^{226}$ which is given by

$$
\Delta G_{m i x}(x)=\Delta H_{m i x}(x)-T \Delta S_{m i x}(x),
$$

where $x$ is the molar fraction of the component $\mathrm{B}$ in the substitutional solid solution $A_{1-x} B_{x}$. From this equation, we need to calculate $\Delta H_{m i x}(x)$ and $\Delta S_{m i x}(x)$, so that the $\Delta G_{m i x}(x)$ can be mapped out. First we start with $\Delta H_{m i x}(x)$ which is defined as

$$
\Delta H_{m i x}(x)=H_{\mathrm{A}_{1-x} \mathrm{~B}_{x}}-(1-x) H_{A}-x H_{B},
$$

where $H_{\mathrm{A}_{1-x} \mathrm{~B}_{x}}$ is the enthalpy of solid solutions, $H_{\mathrm{A}}$ is the enthalpy of $A$ and $H_{\mathrm{B}}$ is the enthalpy of $B$. The enthalpy can be obtain from total energies calculations at equilibrium volume (zero pressure), ${ }^{226} H=E$. Note that the phase stability determination, not only we compare $\Delta G_{m i x}(x)$ with any combination of other concentrations, but we need to compare with any possible ordered states as well. The enthalpy for the pure $A$ and $B$ elements is straight forward to obtained by performing unit cell DFT calculations. But the enthalpy of solid solution is not easy, especially the case of disordered solid solutions due to the unit cell is not enough to simulate the solid solution composition. And standard DFT formalism is builded on the Bloch's theorem (periodic boundary condition), thus it cannot be used to calculate the properties due to translational symmetry is invalid in such a configurational disordered system as the solid solutions. To get around this issue we need to create a supercell (consist of repeated unit cells), and it need to be large enough so that it truly represent the relevant features of disordered solid solution even with the translational symmetry of the supercell. But too large supercell is not preferred due to computer resources limitations. Thus we need a small enough supercell that can still capture the basic properties of disordered solid solution and possible to simulate calculation in super computer in a reasonable time. In order to create such a supercell is not easy, since the atoms in disordered solid solution need to be stochastically placed around the lattice point. We will discuss on how to create such a supercell in the coming section.

The knowledge of temperature dependent $\Delta G_{m i x}(x)$ is important in thermoelectric materials development, since the materials have to be stable in a large temperature gradient. Therefore 
we have to consider the $\Delta S_{m i x}(x)$, in the case of solid solutions, due to there are many contributions for $\Delta S_{m i x}(x)$ i.e., configurational entropy $\Delta S_{m i x}^{\text {conf }}(x)$, vibrational (phonon) entropy $\Delta S_{m i x}^{\text {phonon }}(x)$, magnetic entropy $\Delta S_{m i x}^{\text {mag }}(x)$, and electronic (electrons) entropy $\Delta S_{m i x}^{\mathrm{el}}(x)$. There exist several methods to calculate these entropy, for example, the vibrational free energy for ordered compounds or metals. But it is still difficult to calculate for the disordered solid solution case due to periodic boundary condition violation. We will discuss this topic in the next section about lattice dynamic calculation. Despite of this issue, we are still possible to make approximation to $\Delta S_{m i x}(x)$, so that we can get a reasonable trend of temperature dependent $\Delta G_{\text {mix }}(x)$. In the disordered solid solution, we can consider that the configurational entropy is dominating. Since the typical synthesis or working temperature of nitrides is low compare to their melting temperature ( $T \ll T_{\text {melt }}$ ), thus the contribution from mixing vibrational entropy is rather small $\Delta S_{m i x}^{\text {phonon }}(x) \rightarrow 0$, we can neglect it. Next, the contribution from magnetic mixing entropy can also be neglected due to magnetic entropy to first order, is linear expression with the composition, thus they cancel each other in the mixing term $\Delta S_{\text {mix }}^{\mathrm{mag}}(x) \approx 0$. The last mixing entropy contribution that we can neglect is electronic mixing entropy due to small contribution at low temperature (see ref. [229]). By considering only configurational entropy within the simple mean field approximation,

$$
\Delta S_{m i x}=-k_{\mathrm{B}}(x \ln (x)+(1-x) \ln (1-x)),
$$

where $k_{\mathrm{B}}$ is the Boltzmann constant. However, the configurational degree of freedom can be modeled much more accurately, something that is needed for the creation of a supercell describing the disordered solid solution.

\subsubsection{Configurational Modeling of solid solution}

In this section, we will follow the review article from Ruban and Abrikosov. ${ }^{230}$ To make it easy to illustrate, we consider our fictive $A_{1-x} B_{x}$ solid solution. The configurational interaction between $A$ and $B$ atoms can determine the possibility arrangement between $A$ and $B$ atoms in the lattice. Considering the case when the chemical bonds between $A-A$ and $B-B$ atoms are preferred over $A$ - $B$ bonds, meaning $A$ atoms and $B$ atoms preferably 
stay close to atoms of the same kind. This results in segregation between $A$ and $B$ atoms or clustering (Figure 4.3(a)). On the other hand, if the bond between $A-B$ atoms preferred, the situation changes i.e., now $\mathrm{A}$ and $\mathrm{B}$ atoms like to stay close to each other yielding the ordered solid solution (Figure 4.3(b)). And the last situation when, neither bonds $A-A$ and $B-B$ or $A-B$ are dominated, this will results in the disordered solid solution (Figure 4.3(c)). For the ideal disordered solid solution, we can define a system of atomic configuration that satisfy the condition $V / k_{\mathrm{B}} T \rightarrow 0$, where $V$ is the strongest effective configurational interaction in the system and $T$ is the temperature. The above schematic description is simple but Sanchez, Ducastelle, and Gratias developed a beautiful mathematical formalism for the concept of cluster expansion of the configurational part of the total energy. ${ }^{231,232}$

They take the concept of spin variable, $\sigma_{\mathbf{i}}$ to denote the atomic configuration. For our fictive $A_{1-x} B_{x}$ solid solution, at site $i$ we set $\sigma_{i}$ equal to +1 if that site $i$ is taken by atom $A$ and -1 if it is taken by atom $B$. Thus for a cluster with $N$ sites, we can write the vector that constants all the site atomic configurational spin variables as $\boldsymbol{\sigma}=\left\{\sigma_{1}, \sigma_{2}, \sigma_{3}, \ldots, \sigma_{N}\right\}$. Thus we can span the atomic (a)

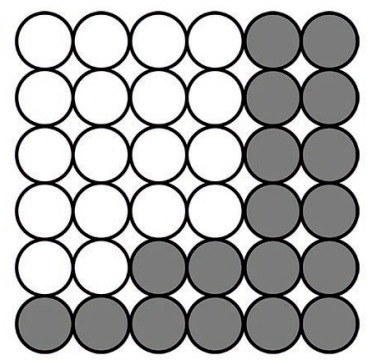

(b)

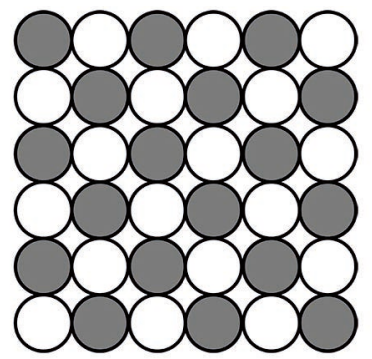

(c)

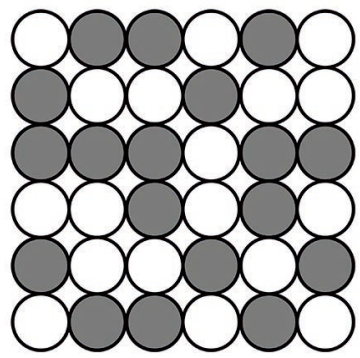

FIGURE 4.3: Illustration of the two-dimensional configuration ordering in a binary system $\mathrm{A}_{0.5} \mathrm{~B}_{0.5}$, (a) Clustering of atoms into a phase segregated two-phase system, (b) Ordered compound, and (c) Disordered solid solution. 
configuration space for a cluster $f$ with the basis functions:

$$
\Phi_{f}^{(n)}(\boldsymbol{\sigma})=\prod_{i \in f} \sigma_{i}
$$

which are defined for a given $n$ site cluster as a product of site atomic configurational spin variables $\sigma_{i}$. Since it is basis functions, it needs to satisfy a complete and orthogonal set, thus

$$
\left\langle\Phi_{\alpha}^{(n)}(\boldsymbol{\sigma}) \mid \Phi_{\beta}^{(n)}(\boldsymbol{\sigma})\right\rangle=\frac{1}{2^{n}} \sum_{\sigma} \Phi_{\alpha}^{(n)}(\boldsymbol{\sigma}) \Phi_{\beta}^{(n)}(\boldsymbol{\sigma})=\delta_{\alpha \beta},
$$

where $\delta_{\alpha, \beta}$ is Kreonecker delta, that is if two clusters differs by at least one site, this inner product is equal to 0 . It is 1 if they are the same. From this basis functions, we can define the cluster correlation functions, $\xi_{f}^{(n)}(\sigma)$, which defines the average value of the cluster function for symmetrically equivalent clusters in any given configuration $\sigma$ as

$$
\xi_{f}^{(n)}(\boldsymbol{\sigma})=\left\langle\Phi_{f}^{(n)}\right\rangle=\frac{1}{m_{f}^{(n)}} \sum_{\alpha \in f} \Phi_{\alpha}^{(n)}(\boldsymbol{\sigma}),
$$

where $m_{f}^{(n)}$ is the normalization factor. Therefore, we can obtain the configuration total energies from

$$
E_{t o t}=\sum_{f} V_{f}^{(n)} m_{f}^{(n)} \xi_{f}^{(n)}
$$

where $V_{f}^{(n)}$ is the configurational effective cluster interactions. From the Equation (4.36), Zunger et al. developed the method to create a supercell that can resemble the solid solution with a reasonable supercell size which is called Special Quasi-random Structure (SQS). ${ }^{233}$ This concept is based on the observation that short ranged and few sites clusters are typically more important than long ranged and multisite clusters when it coms to describing configurational energetics. Therefore we have to arrange our atoms inside supercell so that it give $\xi_{f}^{(n)}(\sigma)$ equal to solid solution ( 0 for disordered solid solution). Ideally this condition needs to satisfy all clusters $f$, however in reality this is not possible due to the periodic boundary of the supercell. Therefore, we have to create supercell that the atomic configuration give $\xi_{f}^{(n)}(\sigma)=0$ from the short range pair clusters up to as many clusters as possible in order to get the best SQS that represent the disordered solid solution. for a given supercell size. 
Since SQS supercell closely represents solid solution structure, therefore this allows us gain information about our solid solution, i.e., the effect of electrostatic energy due to charge transfer and the exact atomic position after lattice relaxation due to atoms at different local environments will locally move away from their fixed lattice point to lower their energy. Nevertheless, one has to be careful when generating the SQS supercell since the $V_{f}^{(n)}$ is typically never known in advance. If there is an alloy or solid solutions compound system that long range interactions are dominated, meaning that our SQS strategy becomes problematic. Therefore, we can say that there is no supercell can truly represent solid solution unless we obtain the knowledge about $V_{f}^{(n)}$ and match the cluster correlation functions for all relevant clusters in the solid solution structure. 


\subsection{Molecular Dynamics}

The molecular dynamics (MD) constitutes apart of the theoretical methodology that is used to calculate material properties in this thesis. The advantage of MD is it provides the knowledge of dynamic of the system i.e., positions and velocities of the atoms, forces, and energies. Since it is possible to connect temperature aspects into MD calculation, we can obtain the temperature dependent of materials properties or study thermodynamic of the system beyond the reach of static density functional theory (DFT) calculation. In this thesis we will use MD to obtain the phonon (lattice dynamic) properties of scandium nitride.

\subsubsection{Basic molecular dynamics formalism}

The molecular dynamics formalism can be derived from the timedependent Schrödinger equation (Equation (4.1)) based on two main approximations i.e., the Born-Oppenheimer approximation and the classical approximation that treats the nuclei as classical point particles. As we have discussed earlier, the DFT typically uses the Born-Oppenheimer approximation to neglect the motion of nuclei when solving the quantum mechanical problem of the electrons. The situation in molecular dynamics is the other side of the story. The Born-Oppenheimer approximation is used to decouple the dynamic of electrons from the nuclei. This approximation leads to the separation ansatz for the fast and slow variables that introduced by Born in 1951. ${ }^{183,234}$ The separation ansatz states that the system wavefunctions can be expressed as

$$
\Phi_{B O}\left(\mathbf{r}_{i}, \mathbf{R}_{I} ; t\right)=\sum_{k=0}^{\infty} \psi_{k}\left(\mathbf{r}_{i}, \mathbf{R}_{I}\right) \chi_{k}\left(\mathbf{r}_{i}, \mathbf{R}_{I} ; t\right),
$$

where the nuclei wavefunctions at energy state $k\left(\chi_{k}\left(\mathbf{r}_{i}, \mathbf{R}_{I} ; t\right)\right)$ and electrons wavefunctions at energy state $k\left(\psi_{k}\left(\mathbf{r}_{i}, \mathbf{R}_{I}\right)\right)$ are normalized to unity, i.e., $\left\langle\chi_{k}\left(\mathbf{r}_{i} \mathbf{R}_{I}\right) ; t \mid \chi_{k}\left(\mathbf{r}_{i} \mathbf{R}_{I}\right) ; t\right\rangle=1$ and $\left\langle\psi_{k}\left(\mathbf{r}_{i} \mathbf{R}_{I}\right) \mid \psi_{k}\left(\mathbf{r}_{i} \mathbf{R}_{I}\right)\right\rangle=1$. 
Consider the many-body Hamiltonian,

$$
\begin{aligned}
\hat{H}= & -\frac{\hbar^{2}}{2 M_{I}} \sum_{I} \nabla_{I}^{2}-\frac{\hbar^{2}}{2 m_{e}} \sum_{i} \nabla_{i}^{2}-\sum_{i, l} \frac{Z_{l} e^{2}}{\left|\mathbf{r}_{i}-\mathbf{R}_{l}\right|} \\
& +\frac{1}{2} \sum_{i \neq j} \frac{e^{2}}{\left|\mathbf{r}_{i}-\mathbf{r}_{j}\right|}+\frac{1}{2} \sum_{l \neq k} \frac{Z_{l} Z_{k} e^{2}}{\left|\mathbf{R}_{l}-\mathbf{R}_{k}\right|} \\
= & -\frac{\hbar^{2}}{2 M_{I}} \sum_{I} \nabla_{I}^{2}-\hat{H}_{e}\left(\mathbf{r}_{i}, \mathbf{R}_{I}\right)-\frac{1}{2} \sum_{l \neq k} \frac{Z_{l} Z_{k} e^{2}}{\left|\mathbf{R}_{l}-\mathbf{R}_{k}\right|} \\
= & -\frac{\hbar^{2}}{2 M_{I}} \sum_{I} \nabla_{I}^{2}-\hat{H}_{e}\left(\mathbf{r}_{i}, \mathbf{R}_{I}\right)-\hat{V}_{I I},
\end{aligned}
$$

and operate this Hamiltonian on $\Phi_{B O}$, multiply $\psi_{k}^{*}\left(\mathbf{r}_{i}, \mathbf{R}_{I}\right)$ to the left, integrate over all electronic space $\left\{\mathbf{r}_{i}\right\}$, and neglect the coupling term between nuclei and electrons we got

$$
\left[-\sum_{I} \frac{\hbar^{2}}{2 M_{I}} \nabla_{I}^{2}+E_{k}\left(\mathbf{R}_{I}\right)+E_{I I}\left(\mathbf{R}_{I}\right)\right] \chi_{k}=i \hbar \frac{\partial}{\partial t} \chi_{k},
$$

where $E_{k}\left(\mathbf{R}_{I}\right)$ is obtained from time-independent electronic Schrödinger equations

$$
\hat{H}_{e}\left(\mathbf{r}_{i}, \mathbf{R}_{I}\right) \psi_{k}\left(\mathbf{r}_{i}, \mathbf{R}_{I}\right)=E_{k}\left(\mathbf{R}_{I}\right) \psi_{k}\left(\mathbf{r}_{i}, \mathbf{R}_{I}\right),
$$

and $E_{I I}\left(\mathbf{R}_{I}\right)$ is is the energy from interaction between nuclei. Next we can approximate the nuclei wavefunction as a classical point particles by taking the classical limit $\hbar \rightarrow 0$. This results in the Equation (4.39) can be identify as equations of motion in the Hamilton-Jacobi formulation of the classical mechanics. And the equations of motion for each atoms $I$ can be determined by

$M_{I} \ddot{\mathbf{R}}_{I}(t)=-\nabla_{I} \int d \mathbf{r} \psi_{k}^{*} \hat{H}\left(\mathbf{r}_{i}, \mathbf{R}_{I}\right) \psi_{k}=-\nabla_{I}\left\langle\psi_{k}\left|\hat{H}\left(\mathbf{r}_{i}, \mathbf{R}_{I}\right)\right| \psi_{k}\right\rangle$.

For the details of this derivation of this equation, see by Marx and Hutter. ${ }^{235,236}$ According to the definition of classical force, $\mathbf{F}_{I}=-\nabla_{I}\left\langle\psi_{k}\left|\hat{H}\left(\mathbf{r}_{i}, \mathbf{R}_{I}\right)\right| \psi_{k}\right\rangle$ where and the Equation (4.41), we arrive at the most notably Newtonian equations of motion for the nuclei that are classically moved by an effective potential surface (global potential energy surface) $V^{E}$ due to electrons quantum mechanic interactions,

$$
M_{I} \ddot{\mathbf{R}}_{I}(t)=-\nabla_{I} V^{E}=\mathbf{F}_{I},
$$

Here we define $V^{E}\left(\mathbf{R}_{I}\right)=\left\langle\psi_{k}\left|\hat{H}\left(\mathbf{r}_{i}, \mathbf{R}_{I}\right)\right| \psi_{k}\right\rangle$. This equation is the central equation of molecular dynamics calculations, because 
it describes the classical trajectories (time evolution of position and velocity) of atoms on the phase space with which the physical properties of the system can be mapped out. ${ }^{237}$

If one carefully enough, one can realize that at this point we are back to the original issue i.e., we have to solve again manybody problems $V^{E}\left(\mathbf{R}_{I}\right)=\left\langle\psi_{k}\left|\hat{H}\left(\mathbf{r}_{i}, \mathbf{R}_{I}\right)\right| \psi_{k}\right\rangle$ to get the force $\mathbf{F}_{I}$, which the number of degree of freedom increase with the number of nuclei. Nevertheless, there is a way to get around this issue. The traditional way of getting around this problem is that we approximate $V^{E}$ as

$$
\begin{aligned}
V^{E} \approx V^{F F}= & \sum_{I}^{N} v_{1}\left(\mathbf{R}_{I}\right)+\sum_{I \neq J}^{N} v_{2}\left(\mathbf{R}_{I}, \mathbf{R}_{J}\right) \\
& +\sum_{I \neq J \neq K}^{N} v_{3}\left(\mathbf{R}_{I}, \mathbf{R}_{J}, \mathbf{R}_{K}\right)+\ldots
\end{aligned}
$$

is often called a "force field" potential. Now our degrees of freedom are reduced from number of electrons into the set of interaction potentials $\left\{v_{i}\right\}$. They can empirically fit from the classical potential e.g. Lennard-Jones potential, ${ }^{37}$ Stillinger-Weber potential, ${ }^{238}$ or Tersoff potential. ${ }^{239}$ This type of molecular dynamics is called classical molecular dynamics. The advantage of classical molecular dynamics is that it uses less computational resources thus the calculation time is fast. But it requires a hard work on parameter fitting since the accuracy of calculation is determined by accurate force field potential approximation. And this make the classical molecular dynamics has less ab initio aspect.

Due to the development of DFT, we can use it to obtain the $V^{E}$ without parameter fitting. With this method makes MD has a fully $a b$ initio aspect which can be called the $a b$ initio molecular dynamics. The Born-Oppenheimer molecular dynamics (BOMD) is a type of $a b$ initio molecular dynamics (AIMD). Here, we applied the Born-Oppenheimer approximation to separate the electron and nucleus dynamics by considering electrons have enough time to relax to the equilibrium state before we move nuclei. At the instance of time where all the nuclei have fixed their positions, we can use static DFT to calculate $V^{E}$ from the electronic structure problem $V^{E}\left(\mathbf{R}_{I}\right)=\left\langle\psi_{k}\left|\hat{H}\left(\mathbf{r}_{i}, \mathbf{R}_{I}\right)\right| \psi_{k}\right\rangle$, after obtaining the potentials from electronic structure, we use them to propagate all the nuclei via classical molecular dynamics. Let consider the 
Equation (4.41), To find the force that propagate the nuclei we consider Hellmann-Feynmann theorem, ${ }^{240}$

$$
\begin{aligned}
\mathbf{F}_{I} & =-\nabla_{I}\langle\psi|\hat{H}| \psi\rangle \\
& =-\left\langle\nabla_{I} \psi|\hat{H}| \psi\right\rangle-\left\langle\psi|\hat{H}| \nabla_{I} \psi\right\rangle-\left\langle\psi\left|\nabla_{I} \hat{H}\right| \psi\right\rangle .
\end{aligned}
$$

If the eigenvectors $|\psi\rangle$ are a complete basis set and purely independent of nuclei positions, due to the orthonormality of the eigenvectors the first and second term of above equation are vanished. Thus we can deduce Hellmann-Feynmann force,

$$
\mathbf{F}_{I}^{\mathrm{HF}}=-\left\langle\psi\left|\nabla_{I} \hat{H}\right| \psi\right\rangle=-\left\langle\psi\left|\frac{\partial H\left(\mathbf{R}_{I}\right)}{\partial \mathbf{R}_{I}}\right| \psi\right\rangle .
$$

According to many-body Hamiltonian (Equation (4.38)), we can obtain its derivative with respect to $\mathbf{R}_{I}$,

$$
\frac{\partial H\left(\mathbf{R}_{I}\right)}{\partial \mathbf{R}_{I}}=\frac{1}{2} \sum_{I \neq k} \nabla_{I} \frac{Z_{I} Z_{k} e^{2}}{\left|\mathbf{R}_{I}-\mathbf{R}_{k}\right|}-\sum_{i, I} \nabla_{I} \frac{Z_{I} e^{2}}{\left|\mathbf{r}_{i}-\mathbf{R}_{I}\right|},
$$

and the Hellmann-Feynmann force is given by

$$
\begin{aligned}
\mathbf{F}_{I}^{\mathrm{HF}}= & -\left\langle\psi\left|\frac{\partial H\left(\mathbf{R}_{I}\right)}{\partial \mathbf{R}_{I}}\right| \psi\right\rangle \\
= & -\nabla_{I}\left[\frac{1}{2} \sum_{I \neq k} \frac{Z_{I} Z_{k} e^{2}}{\left|\mathbf{R}_{I}-\mathbf{R}_{k}\right|}\right. \\
& \left.+\sum_{I} \int \psi^{*}\left(\mathbf{r}_{1} \ldots \mathbf{r}_{M}\right) \frac{Z_{I} e^{2}}{\left|\mathbf{r}_{i}-\mathbf{R}_{I}\right|} \psi\left(\mathbf{r}_{1} \ldots \mathbf{r}_{M}\right) d \mathbf{r}_{1} \ldots d \mathbf{r}_{M}\right] \\
= & -\frac{1}{2} \sum_{I \neq k} \nabla_{I} \frac{Z_{I} Z_{k} e^{2}}{\left|\mathbf{R}_{I}-\mathbf{R}_{k}\right|} \\
& -\nabla_{I}\left[\sum_{i} \int n\left(\mathbf{r} ;\left\{\mathbf{R}_{I}\right\}\right) \frac{Z_{I} e^{2}}{\left|\mathbf{r}_{i}-\mathbf{R}_{I}\right|} d \mathbf{r}\right] \\
= & -\frac{1}{2} \sum_{I \neq k} \nabla_{I} \frac{Z_{I} Z_{k} e^{2}}{\left|\mathbf{R}_{I}-\mathbf{R}_{k}\right|}-\sum_{i} \int n\left(\mathbf{r} ;\left\{\mathbf{R}_{I}\right\}\right) \nabla_{I} \frac{Z_{I} e^{2}}{\left|\mathbf{r}_{i}-\mathbf{R}_{I}\right|} d \mathbf{r} \\
& -\sum_{i} \int\left(\nabla_{I} n\left(\mathbf{r} ;\left\{\mathbf{R}_{I}\right\}\right)\right) \frac{Z_{I} e^{2}}{\left|\mathbf{r}_{i}-\mathbf{R}_{I}\right|} d \mathbf{r}
\end{aligned}
$$

where $n(\mathbf{r})$ is the electron density and If the electron density is fully self-consistent, the last term should be vanished. Thus the the Hellmann-Feynmann force can be written as

$$
\mathbf{F}_{I}^{\mathrm{HF}}=-\frac{1}{2} \sum_{I \neq k} \nabla_{I} \frac{Z_{I} Z_{k} e^{2}}{\left|\mathbf{R}_{I}-\mathbf{R}_{k}\right|}-\sum_{i} \int n\left(\mathbf{r} ;\left\{\mathbf{R}_{I}\right\}\right) \nabla_{I} \frac{Z_{I} e^{2}}{\left|\mathbf{r}_{i}-\mathbf{R}_{I}\right|} d \mathbf{r}
$$


However, we need to rely on the numerical calculations to determine the force, thus there is no guarantee that we can obtain fully self-consistency due to numerical uncertainty. Thus the last term of above equation will not disappear, so that the correction term need to be added to the force explicitly. Therefore, the corrected force that acts on the nuclei can be written as,

$$
\mathbf{F}_{I}=\mathbf{F}_{I}^{\mathrm{HF}}+\mathbf{F}_{I}^{\mathrm{NSC}},
$$

where $\mathbf{F}_{I}^{\mathrm{NSC}}$ is the "non-self-consistent correction" (NSC) force which is given by

$$
\mathbf{F}_{I}^{\mathrm{NSC}}=\int\left(\nabla_{I} n\left(\mathbf{r} ;\left\{\mathbf{R}_{I}\right\}\right)\right)\left(V^{\mathrm{SCF}}-V^{\mathrm{NSC}}\right) d \mathbf{r},
$$

where $V^{\mathrm{SCF}}$ is the self-consistent potential and $V^{\mathrm{NSC}}$ is the nonself-consistent potential. In addition, if our eigenvector is an incomplete basis set and depended on the position of nuclei, these lead to the nucleus gradients of the basis functions. Therefore the first and second term of Hellmann-Feynmann equation (Equation (4.44)) are not vanished, resulting in another type of correction force need to be added. This correction force is called "incomplete-basis-set correction" (IBS) $\mathbf{F}_{I}^{\mathrm{IBS}}$ in the solid state theory and corresponds to the "wavefunction force" or "Pulay force" in quantum chemistry. Luckily, we can neglect this force by choosing the basis set that is independent with the position of nuclei such as plane waves basis set. According to the Equation (4.49), we can define the $V^{B O}$ as

$$
-\nabla_{I} V_{e q}^{B O}=-\left\langle\psi_{e q}\left|\nabla_{I} \hat{H}\right| \psi_{e q}\right\rangle=\left(\mathbf{F}_{I}^{\mathrm{HF}}+\mathbf{F}_{I}^{\mathrm{NSC}}\right)=\mathbf{F}_{I}
$$

According to the definition of $V^{B O}$, it is easy to obtain by DFT calculations. We can consider $\left\langle\psi\left|\nabla_{I} \hat{V}^{B O}\right| \psi\right\rangle$ equal to system energy functional $E_{\mathrm{DFT}}[n(\mathbf{r}),\{\mathbf{R}\}]$. Thus the force that act on the nuclei will equal to

$$
\begin{aligned}
\mathbf{F}_{I} & =-\frac{d E_{\mathrm{DFT}}[n(\mathbf{r}),\{\mathbf{R}\}]}{d \mathbf{R}_{I}} \\
& =-\frac{\partial E_{\mathrm{DFT}}[n(\mathbf{r}),\{\mathbf{R}\}]}{\partial \mathbf{R}_{I}}-\frac{\partial E_{\mathrm{DFT}}[n(\mathbf{r}),\{\mathbf{R}\}]}{\partial \mathbf{R}_{I}} \frac{\delta n(\mathbf{r})}{\delta \mathbf{R}_{I}}
\end{aligned}
$$

This gives Born-Oppenheimer molecular dynamics (BOMD) higher accuracy than the classical molecular dynamics but it also requires a lot of computer resource in order to preform the calculations, because the BOMD is needed well convergent self-consistent calculation to eliminate the non-self-consistent correction. Nevertheless, the advance in computational calculation scheme and 
the growth in computer allows the ab initio molecular dynamics can be performed. The BOMD calculations have been used to determine the interatomic force constant (IFC) to determine the phonon thermal conductivity of $\mathrm{ScN}$ in this Thesis.

\subsubsection{Time integration algorithm}

According to the many-body nature of the problem, we cannot analytically solve the equations of motion (Equation (4.42)). Thus they need to be discretized and solved numerically by computer calculations. To solve these second order differential equations the proper time integration algorithm is required. ${ }^{181,182}$ The important property of time integration algorithm is that the accurate time reversibility. Because in MD the trajectory of the system should remain in the phase space that is constrained by the total energy of the system. This means if we integrate the system from $t$ to $t+\Delta t$ then go back in time again, the system should go back to exactly the same position and velocity from where they start. Otherwise the initial phase space is not conserve resulting in the shift in the phase space volume is called a drift. ${ }^{181}$ Consequently, this drift will cause the loss of energy conservation which is call energy drift. ${ }^{181}$ The energy drift can be categorized into two, short time and long time conservation. As stated, the short time conservation means the system total energy conserved over a short time then start to diverge in a long time. And it is opposite for long time conservation. These drifts are unavoidable due to numerical errors and to the discretization. Therefore a good time integration algorithm should minimal exhibit such drifts. The Verlet algorithm is commonly used in MD calculations due to its numerical stability and simplicity to implement making the calculation efficient. ${ }^{237}$ In the velocity Verlet algorithm, ${ }^{241}$ the positions and velocities are given by

$$
\begin{aligned}
& \mathbf{R}_{I}(t+\Delta t)=\mathbf{R}_{I}(t)+\dot{\mathbf{R}}_{I}(t) \Delta t+\frac{1}{2 M_{I}} \mathbf{R}_{I}(t) \Delta t^{2}, \\
& \dot{\mathbf{R}}_{I}(t+\Delta t)=\dot{\mathbf{R}}_{I}(t)+\frac{\mathbf{F}_{I}(t+\Delta t)+\mathbf{F}_{I}(t)}{2 M_{I}} \Delta t,
\end{aligned}
$$

where $\Delta t$ is the step discretizing time, $\dot{\mathbf{R}}_{I}(t)$ is the velocities $\mathbf{V}_{I}(t)$, and $\mathbf{F}_{I}(\mathrm{t})$ is the forces acting on atom $I$. The velocities from this method need to calculate in two steps i.e., first from the force at time $t$ and then calculate a new force at time $t+\Delta t$ from the new positions $\mathbf{R}_{I}(t+\Delta t)$. To integrate (or propagate) the 
system from time $t$ to time $t+\Delta t$, the instantaneous forces acting on atoms are given by DFT calculations and the initial positions and velocities need to be specified.

The choice of size of time step is also important to prevent the energy drift. Too large a time step can cause the system total energies diverge over time which is often refers as "exploding" since we can observe the large shift in atomic positions from the equilibrium positions. Typically the rule of choosing size of time step is choose according to the fastest movement in the system i.e., $\Delta t \sim\left(\frac{0.01}{\left.0.1 \Delta t_{\max }\right)}\right.$ where $\Delta t_{\max }$ is the period of the fastest phonon mode: $\Delta t_{\max }=1 / \omega_{\max } \cdot{ }^{210}$ For example, the time step of $1 \mathrm{fs}$ is used to calculated the thermal properties for $\mathrm{ScN}$ in my thesis since the typical time step for transition metal nitride is around 1$2 f$ s. After an integration with appropriate number of time steps, the properties of interest are gathered.

\subsubsection{Thermodynamical averages in molecular dynamics}

Despite the fact that molecular dynamics is based on Newton's equation of motion, we cannot however assume that MD is a deterministic method. This is because we cannot analytically solve those equations of motion as we discussed in previous section (Section (4.7.2)). Due to the numerical error and the discretization, the MD calculations are sensitive to the initial parameters resulting in the trajectories in each MD calculation not being relevant by itself. Therefore the MD calculation is actually considered as a statistical method to be considered as theoretical experiment. The integration algorithm suggests that after each integration round we obtain a set of $3 N$ positions and $3 N$ velocities which defines a point (configuration) in the $6 N$-dimensional phase space for the system consists of $N$ atoms. After calculation finished, the MD trajectory gives a set of configurations that sample through the phase space along a contour of constant energy. Since these configurations are connected with time, so the statistical mechanics according to ergodic principle suggest that we can replace the ensemble average of an ergodic system by a time average to obtain the macroscopic physical quantity in thermal equilibrium as

$$
\langle a\rangle=\frac{\int a(\{\mathbf{p}\},\{\mathbf{q}\}) \exp (-\beta H(\{\mathbf{p}\},\{\mathbf{q}\})) d\{\mathbf{p}\} d\{\mathbf{p}\}}{\int \exp (-\beta H(\{\mathbf{p}\},\{\mathbf{q}\})) d\{\mathbf{p}\} d\{\mathbf{p}\}}
$$


where $a(\{p\},\{q\})$ is the interested physical property as a function of $3 N$ momentum conjugates $\{\mathbf{p}\}$ and $3 N$ generalized coordinates $\{\mathbf{q}\}$ and $H(\{\mathbf{p}\},\{\mathbf{q}\})$ is a classical Hamiltonian of the system. Note that here I write only one integral sign instead of $6 N$ for a convenient. This equation can be replaced by

$$
\langle a\rangle=\lim _{N_{t} \rightarrow \infty} \frac{1}{N_{t}} \sum_{t=1}^{N_{t}} a(t)
$$

where $N_{t}$ is a number of time step and $a(t)$ is the time dependent interested physical property (see ref. [242] for more details discussion on ergodicity). The ergodic system means at sufficiently long periods of time, the system should pass though all microstates (points) in the phase space, thus we can say all the accessible microstates are equiprobable. This statement give an impression that only one or a few MD calculations are enough to get the ergodic system. Since MD calculation exhibits a sensitivity to initial conditions as discussed in the previous section (Section (4.7.2)) and this sensitivity will lead the system be ergodic after certain amount of simulation time (number of timestep). This is true for the large size system (millions or billions of particles) which can be done in classical MD. Because the large size system can cause a large numerical fluctuation which helps the system reach ergodicity. However one must be careful when considering small size system with particle numbers ranging from the tens to the hundreds. Since, the small system leads to low numerical fluctuation thus sometime after a few MD calculations we may end up with the system that do not fully represent ergodicity i.e., the MD calculation only sample a certain part in phase space. When this situation happens the properties derived from this MD calculations do not converge to the same average values for the properties as a full ergodic calculation.

Another issue that needs to be pointed out is when we sampling on harmonic or near harmonic potential. The harmonic or near harmonic potential can cause the situation where the trajectories of the system are fixed at certain area in the phase space so called "mode-locking". ${ }^{180}$ This will lead to the non-ergodic system. The small size system will suffer more than large size system due to smaller influence of the numerical fluctuation. To sample phase space in this situation, one can run many MD calculations with slightly different in the initial conditions. However this method requires long waiting time to obtain the results and sometimes 
more calculations are needed. To get around these issues we introduce the stochastic thermostat into the system which can break the mode-locking. This thermostat will be discussed in the next Section. As the key point in MD calculations is that the system need to be ergodic so that we can obtain meaning full physical properties. Thus if we have a potential in question, we can use the method that was developed by O. Hellman to monitor the system ergodicity. ${ }^{180}$

\subsubsection{Thermostat}

MD calculations yield the microcanonical (constant $N V E$ ) ensemble, since they obey the Newtonian dynamics which imply the energy conservation and are typically performed in a constant volume $V$ and number of atoms $N$. To study the temperature dependent processes, one must have knowledge on temperature of the system. However the determination of temperature from averaging system thermal energy over microcanonical ensemble is not trivial. Since a microcanonical ensemble with a finite number of particles, the instantaneous temperature will fluctuate and start to diverge over time. Moreover, considering in microcanonical ensemble will leads to many disadvantages for example, (i) one lose the experimental aspect since most of experiments are not performed in isolated system; (ii) one cannot calculate the transport coefficients due to viscous- or heat-flow since unable to evacuate the heat in dissipative non- equilibrium MD calculations; (iii) one cannot avoid the steady energy drift due to the numerical errors accumulation when it is impossible to avoid by timestep $\Delta t$ reduction or shorten the number of timestep, $N_{t}{ }^{\mathrm{IX}}$.

Instead one can perform the MD calculations that yields canonical (constant $N V T$ ) ensemble where the temperature can be controlled by exchanging energy of the system with environment via thermostat algorithms (imagine like heat bath or heat reservoir in thermodynamics sense). To use a thermostat, we need to know the instantaneous temperature of the system at any timepoint, $T(t)$. This temperature will then be compared to the reference

(IX) Note that the minimal energy drift is required for a good quality MD calculations. temperature $T_{0}$ Here the instantaneous temperature, $T(t)$ can be determined by the kinetic energy ${ }^{243}, K$ as

$$
T(t)=\frac{2}{3 k_{\mathrm{B}} N_{a}} K=\frac{2}{3 k_{\mathrm{B}} N} \sum_{i}^{N} \frac{M_{I} \dot{\mathbf{R}}_{I}^{2}}{2},
$$


and the temperature average is

$$
\langle T(t)\rangle_{t}=\frac{2}{3 k_{\mathrm{B}} N_{a}} \sum_{i}^{N}\left\langle\frac{M_{I} \dot{\mathbf{R}}_{I}^{2}}{2}\right\rangle_{t},
$$

where \langle\rangle$_{t}$ defines time average, $k_{\mathrm{B}}$ is the Boltzmann's constant, $M_{I}$ is the atomic mass of atom $I, \dot{\mathbf{R}}_{I}$ is the velocity of atom $I$ and $N$ is the number of atoms in the system. The equation obviously suggests that we need an algorithm that rescale the velocities at every $n$ timestep to match the kinetic energy to the interested temperature $T$ which is called velocities rescaling. Indeed, it is easy to implement but not efficient since it requires to rescale every timestep to generate a canonical ensemble and works only in a system with a large number of particles, which is something we can hardly effort in $a b$ initio molecular dynamics. Moreover the velocity rescaling does not confirm that the averages will be correct due to velocity trajectories are discontinuous at every rescaling step. ${ }^{244}$

The correct way to generate the canonical ensemble (MD trajectories) at interested temperature $T$ is defined a suitable thermostat that couple the system with a heat bath at the temperature $T$ of interest. There are two categories of thermostats i.e., deterministic thermostats and stochastic thermostats. The Andersen thermostat $^{245}$ is a well-known stochastic thermostats. The principle behind this thermostat is introducing a fixed probability to every atom that will collide with the heat reservoir at every timestep. The collision probability is distributed according to Poisson distribution. After the collision, the atoms will gain a new velocities according to Maxwell-Boltzmann distribution which is used to control temperature. Over a series of integrations, the microcanonical ensembles will transform into a canonical ensemble due to non-zero collision frequency. ${ }^{245}$ It is this non-zero collision frequency that gives the stochastic effect to this thermostat, thus it can break the mode-locking.

However it is not good for a small size system ( $~ 100$ atoms) with too high collision frequency giving a discontinuous velocity trajectories. Because the randomly-occuring collisions cause significantly perturbation to the dynamics of the system. Besides, Andersen thermostat also exhibit time-irreversible causing a drift in phase space volume. Therefore a discontinuous velocity trajectories and drift in phase space volume can cause the results of MD 
FIGURE 4.4: Illustration of different molecular dynamics phase space trajectories on a classical harmonic potential surface using different thermostats. Courtesy by $\mathrm{O}$. Hellman ${ }^{180}$. calculations have to be unreliable, so we cannot extract velocities related properties from this calculations. Of course, this issue can be reduced by having low collision frequency, but this is inefficient since the system will take a long simulation time to sample a canonical ensemble. ${ }^{243}$

Another stochastic thermostat is using Langevin's equation of motion instead of Newtons ${ }^{180,243}$ :

$$
M_{I} \ddot{\mathbf{R}}_{I}(t)=\mathbf{F}_{I}(t)-M_{I} \gamma_{I} \dot{\mathbf{R}}_{I}(t)+\xi_{I}(t) .
$$

The original intention of the Langevin's equation of motion is to describe the Brownian motion of a particle in a fluid, so the particle velocity is reduced by the (positive) atomic friction parameter $\gamma_{I}$. The random motion of the particle occurs due to the collisions with the molecule in the fluid giving a random force $\xi_{I}$. At every timestep, the $\xi_{I}$ is distributed to atom $I$ in the system via Gaussian distribution with the standard deviation $\sigma^{2}$ that relate to the $\gamma_{I}$, as $\sigma^{2}=2 \gamma_{I} M_{I} k_{\mathrm{B}} T_{0}$. Thus $\gamma_{I}$ and $\xi_{I}$ control the temperature and stochastic behavior. Since the Langevin's equation of motion is a modified Newton's equation of motion, therefore it can be integrated by a Verlet-like algorithm ${ }^{246}$ resulting in a configurations distribution according to the canonical ensemble.

The Langevin thermostat can in many cases be considered as a superior stochastic thermostat (see Figure 4.4); it can prevent the system from mode-locking and speeding up the phase space sampling. In addition, we can choose a relatively large timestep $\Delta t$ since the atomic friction parameter $\gamma_{I}$ can stabilize the energy drift. This will fasten the MD calculation speed, and in addition it can be integrated by a Verlet-like algorithm. In case of low atomic friction parameter $\gamma_{I}$, the velocity trajectories is smooth so that there is not issue similar to Andersson thermostat in case of small

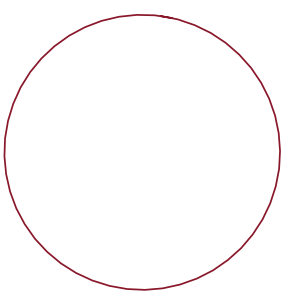

Microcanonical

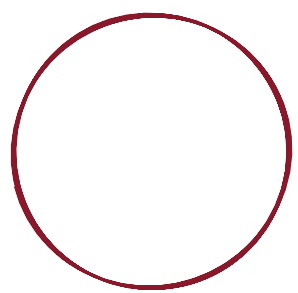

Nosé-Hoover

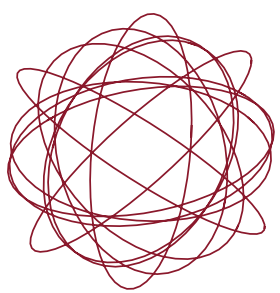

Andersen

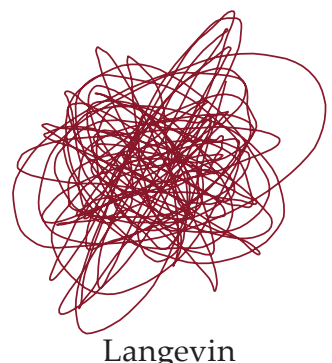

Langevin 
size system. However too strong $\gamma_{I}$ can increase the phase space sampling speed, at the same time it can rise the chance of discontinuous velocity trajectories. Too low $\gamma_{I}$, the system will loss the temperature control. This can be obviously seen if the $\gamma_{I}=0$, the Langevin's equation is back to Newton's equation. So the system configurations are back to microcanonical ensemble (no temperature). These considerations show the disadvantage of Langevin thermostat i.e., both atomic friction parameter $\gamma_{I}$ and a random force $\xi_{I}$ are system specific, as a consequence they need to be predetermined by doing the series of test MD calculations with Langevin thermostat for different $\gamma_{I}$ and $\xi_{I}$. And the Langevin thermostat is time-irreversible thus it generate phase space volume drift, therefore we can extract some physical properties like diffusion coefficient from the system with Langevin thermostat.

The last thermostat that we are going to introduce in this Thesis is the Nosé-Hoover thermostat. ${ }^{210}$ It was first introduced by Nosé ${ }^{247}$ and following developed by Hoover. ${ }^{248}$ And it is the most well-known thermostat since it is straightforward to code. The idea behind this thermostat is a coupling between the equations of motion to an external system with an addition of fictitious mass $Q>0$ and an extra degree of freedom $\zeta$ that controls the temperature. The new equation of motion for system with $N$ atoms can be expressed by

$$
\begin{gathered}
\ddot{\mathbf{R}}_{I}(t)=\frac{F_{I}(t)}{M_{I}}-\dot{\zeta}(t) \dot{\mathbf{R}}_{I}(t), \\
\ddot{\zeta}(t)=\frac{1}{Q}\left[\sum_{i}^{N} M_{I} \dot{\mathbf{R}}_{I}^{2}-3 N k_{\mathrm{B}} T_{0}\right] .
\end{gathered}
$$

These two equations can be discretized and integrate simultaneously during the calculation. Additionally the Equation (4.61) can be seen as the temperature controller, when the $\ddot{\zeta}(\mathrm{t})$ is negative, it gives the heat bath will heat up the system to until the system temperature reach $T_{0}$, the sign in front of $\dot{\zeta}$ in Equation 4.60 is positive. Oppositely, when the $\ddot{\zeta}(\mathrm{t})$ is positive, the heat bath will cool down the system the system temperature decrease to $T_{0}$. Therefore we can consider that Nosé-Hoover equations of motion sample canonical ensemble. ${ }^{248}$ The degree of coupling is depended on the fictitious mass $Q,{ }^{243}$ as if the $Q$ is too large, the system will lose the temperature control. Thus canonical ensemble distributions will be obtained after long calculation time. 
Obviously, when $Q \rightarrow \infty$ we get $\ddot{\zeta}=0$ therefore we back to microcanonical MD equation of motion. On the other hand, $Q$ is too small lead to small heat exchange at each timestep due to a tight coupling which may result in inefficient temperature control. Since there is no random factor in Nosé-Hoover equations of motion, it can be considered as deterministic thermostat. The Nosé-Hoover equations of motion also exhibit smooth and time reversible thus it well suited for small systems since it gives accurate MD trajectories over phase space.

But the oscillating behavior of the MD trajectories in phase space (see Figure 4.4) which is caused by heat flow in and out of the system due to the time-evolution of the $\dot{\zeta}$, results from a second-order differential equation (Equation 4.61). This only weakly disrupts the trajectories and cannot break the mode-locking of harmonic or near harmonic system. ${ }^{248}$ This is something we need to be careful when we extract the physical property average from the MD calculations with Nosé-Hoover thermostat. For the more details discussion about thermostat (see ref. [243]).

\subsubsection{Free energy determination in molecular dynamics}

In molecular dynamics, we typically consider the system configuration distribution according to canonical ensemble (NVT constant). Under these conditions equilibrium correspond to the minimization of the Helmholtz free energy, $F$ will be considered in MD. Unfortunately, the Helmholtz free energy is one of the thermal properties we cannot directly obtain from the MD trajectories (another obvious one is entropy). But one can extract differences in $F$ from a series of calculations for two systems for which the potential energies are known, which is called "thermodynamics integration".

Considering the partition function $\mathcal{Z}$ of the system with $\mathrm{N}$ atoms that has potential energy function of $U(\{\mathbf{r}\})$,

$$
\mathcal{Z}=\frac{1}{\Lambda^{3 N} N !} \int \exp (-\beta U(\{\mathbf{r}\})) d\{\mathbf{r}\}
$$

where $\Lambda$ is a constant and $\{\mathbf{r}\}$ is a set of atomic coordinates $\mathbf{r}$. From this partition function, we get Helmholtz free energy as,

$$
F=-k_{\mathrm{B}} T \ln \mathcal{Z} \text {. }
$$


Then we substitute the potential energy function of $U(\{\mathbf{r}\})$ with "metapotential", $U(\{\mathbf{r}\}, \lambda)$ which the coupling between the two potential energies using a switching parameter, $\lambda$,

$$
U(\{\mathbf{r}\}, \lambda)=(1-\lambda) U_{i}(\{\mathbf{r}\})+\lambda U_{f}(\{\mathbf{r}\}) .
$$

This technique is called The Kirkwood coupling technique. ${ }^{249}$ Then when differentiating the Helmholtz free energy with respect to $\lambda$, we get

$$
\begin{aligned}
\frac{\partial F}{\partial \lambda} & =-k_{\mathrm{B}} T \frac{\partial}{\partial \lambda} \ln \mathcal{Z}(\lambda) \\
& =\frac{k_{\mathrm{B}} T}{\mathcal{Z}(\lambda)} \frac{\partial \mathcal{Z}(\lambda)}{\partial \lambda} \\
& =\frac{\int \frac{\partial U}{\partial \lambda} \exp (-\beta U(\{\mathbf{r}, \lambda\})) d\{\mathbf{r}\}}{\int \exp (-\beta U(\{\mathbf{r}, \lambda\})) d\{\mathbf{r}\}} \\
& =\left\langle\frac{\partial U}{\partial \lambda}\right\rangle
\end{aligned}
$$

where $\left\langle\frac{\partial U}{\partial \lambda}\right\rangle$ is the the ensemble average of the derivative of $U(\{\mathbf{r}\}, \lambda)$ with respect to $\lambda$. And if our systems are ergodic, we can replace the ensemble average to the time average,

$$
\frac{\partial F}{\partial \lambda}=\left\langle\frac{\partial U}{\partial \lambda}\right\rangle_{t}
$$

Then integrate over $\lambda$ from system $i$ to $f$, which $\lambda$ at $i$ and $f$ are given as 0 and 1 , respectively,

$$
F_{f}-F_{i}=\int_{0}^{1}\left\langle U_{f}-U_{i}\right\rangle_{t} d \lambda
$$

Here we get, the free energy difference between system $i$ and $f$ equal to the integral of the time average potential energy difference between system $i$ and $f$. So if we know $U_{i}, U_{f}$, and $F_{i}$, we can integrate to find $F_{f}$. For example, If we select $U_{i}$ is a harmonic potential energy, so that we can exactly obtain the harmonic free energy of that system, $F_{i}=F_{\text {har }}$ from the phonon free energy functional. We can then integrate to the free energy of any system, $F_{f}$, since we can extract potential energy of any system from MD calculation. Some cautions need to be taken, running MD simulations with harmonic potential energy, there is a high chance of mode-locking may occurs. Therefore, a lot of phase space samplings is needed or one can also put atoms in a position of averaging values over calculations time, something that can take very long to converge. This means that the determination free energy by thermodynamic integrations requires long calculation time. 


\subsubsection{Basic molecular dynamic routine}

The basic principle in MD calculation can be considered as Figure 4.5. First we model the system of interest, in this thesis the system consists of a supercell lattice with atoms that mass and charge are assigned are spanned on the lattice point according to basis set. Then, the starting parameter of the system needs to be set, typically the positions and velocities of atoms will be selected according to the temperature that we interest. To select these parameters, there are different ways for example the most common one is choose ideal positions and random velocities, using a gaussian or Maxwell-Boltzmann distribution that corresponding to a certain temperature to assign the velocities, no displacement. This will speed up the phase space sampling in MD calculations.

FIGURE 4.5: Illustration of the basic molecular dynamics calculation routine.

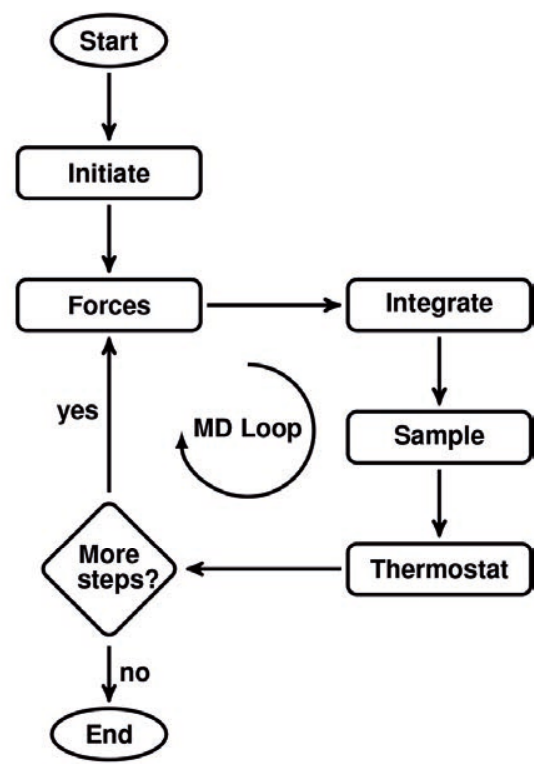

The precise and faster way of assign the initial positions and velocities was suggested by West et al. ${ }^{250}$ They suggested that if we know the harmonic potential of our system, one can assign the initial positions and velocities according to solutions of the eigenvalue problem in lattice dynamics (see Chapter 4) at the temperature of interest. This will minimize or remove the equilibration time in MD calculations. Since there is no the equilibration time, thus this make phase space sampling becomes faster due to parallel calculations which makes the calculation more efficient, less 
computer resource usage (see Section (5.7) in ref. [180]). After that the MD loop starts with two important steps : the force calculation and the positions and velocities integration. The force calculation is where all of the atomic interactions between atoms are calculated.

In AIMD, we obtain these atomic interactions by performing DFT calculations. These forces will used to integrate the atomic equations of motion and propagate all atoms inside supercell. After this step, the physical properties of the system will be calculated. We will obtain positions and velocities of the atoms, potential energies, kinetic energies (temperature). Next step, the temperature of the system will be adjusted by thermostat. It is often applied to the system but not mandatory, because it depends on the specific question of interest. As we discussed in thermostat section (Section (4.7.4)). Finally the system will be checked, if it reach number of time steps that we set. If the system does not reach, we add the time by one step and calculation start over from force calculation at the current atomic positions. This process will continue until the system reach the last time step. Once the calculation is done, all the interested data that was generated per time step will come out as an output. So when we need to do post-processing in order to obtain an interested or meaningful conclusion such as pressure, stress, diffusion coefficients or phase stability. 


\subsection{Force constants calculations}

The ability to calculate and predict lattice thermal conductivity from first principle is vital importance not only for those who are interesting in the field of thermoelectric development. But it also includes various other research fields such as thermal barrier development that requires low thermal conductivity to protect jet engine, or semiconductor and semiconductor packaging which high thermal conductivity is required to have high heat dissipation.

As discussed in Chapter 3, the harmonic approximation show the failures to describe many important thermal properties due to harmonic approximation does not consider the crystal volume change with temperature and neglect the anharmonic contribution resulting in phonons are non-interacting and live forever. For the first issue can be corrected by the quasi-harmonic approximation. ${ }^{251-253}$ In this approximation, the quadratic interatomic potential surface of the crystal, $U(\{\mathbf{R}\}, V)$ is now depended on the crystal volume $V$ as well as a set of atomic positions, $\{\mathbf{R}\}$. To determine the volume dependent phonon frequencies $\omega_{j \mathbf{q}}(V)$, the second order interatomic force constants $\Phi_{i j}^{(2)}(V)$ (where $i, j$ are the atomic indices) at each volume can be determined by the finite displacement method ${ }^{254}$ in zero-temperature DFT calculations where the $\Phi_{i j}^{(2)}(V)$ is approximated by the ratio between forces on all atoms that are induced by displacing on atom and the displacement of that atom. The example of this approach that has been implemented in Phonopy. ${ }^{253,255}$ Another method that can obtain the second-order force constants is called density functional perturbation theory ${ }^{256-258}$ (DFPT) where the second-order force constants can be obtained directly from the electronic calculations. By recalculating interatomic forces with multiple volumes, we will obtain the volume dependent phonon frequencies $\omega_{j \mathbf{q}}(V)$ where the effect of anharmonicity in the crystal partially included into the phonon frequencies. Therefore, the quasi-harmonic approximation can be used to determine constant volume heat capacity $C_{V}$, entropy $S_{\text {vib }}$, Helmholtz free energy $F_{h a m}$, thermal expansion (need the temperature dependent free energy).

Despite all of these successful results, the quasi-harmonic approximation still has some flaws due to fact that (i) the force constants are extracted at $0 \mathrm{~K}$ (DFT or DFPT calculation) meaning that the 
potential energy surface does not depend on temperature which is not true; (ii) the anharmonic effects beyond simple volume expansion is neglected or not fully covered, thus it fails to predict thermal properties of highly anharmonic system; Another weakness of quasi-harmonic approximation which is the consequence of previous point is that (iii) it only considers the $0 \mathrm{~K}$ Taylor expansion of the interatomic potential energy surface around the atomic equilibrium position up to the quadratic term (harmonic approximation), which at the temperatures close to the melting point this assumption becomes inaccurate. Since the atoms in the crystal does not stay at equilibrium position anymore. By omitting these physics in the approximation can lead to a serious issue. If any of the second derivatives in the interatomic force constants matrix result in negative values. These values will appear in the dynamical matrix resulting in it is not a hermitian matrix anymore. Therefore the $\omega$ becomes imaginary (eigenvalue $\omega^{2}$ is negative) which cannot be used to determine Helmholtz free energy meaning that that crystal lattice is dynamically unstable at zero temperature (see Chapter 3 for the formalism). But the fact that it exists in the reality due to the approximation does not take into account the fact that the material can be stabilized by the anharmonic contributions. For example the body-centered cubic iron-nickel alloy ${ }^{259}$ can be stabilized at high temperature, bcc Li that stabilizes at $70 \mathrm{~K}$ whereas the harmonic approximation show an unstable at $0 \mathrm{~K} .{ }^{260}$ Or the failure of quasi-harmonic approximation in predicting of the apparent of avoid crossing between transverse optical phonon branch and longitudinal acoustic branch of PbTe phonon dispersion relation due to strong anharmonic coupling. ${ }^{44}$ To Illustrate all these issues of (quasi-) harmonic approximation, we will follow the discussion in Ph.D Thesis by O. Hellman ${ }^{180}$ in the following section.

\subsubsection{One dimensional anharmonic potential}

We start this section by considering the one dimensional interatomic potential of a fictive crystal :

$$
U(x)=\frac{k\left(x-x_{0}\right)^{2}}{2}+\alpha e^{-\beta\left(x-x_{0}\right)^{2}},
$$

where the first term is a ordinary harmonic potential and the second term is added to make this interatomic potential anharmonic. 
True potential

TDEP

Harmonic ..........

FIGURE 4.6: The plot compares the TDEP and the harmonic approximation for both potential (left hand side panel)and force (right hand side panel) with the one dimensional interatomic potential described by Equation 4.68 where $\alpha$ and $x_{0}$ are the parameters in equation 4.68. The plots show that the TDEP has better fitted than the harmonic approximation. Reprinted figure with permission from ref. [261] @2013 by the American Physical Society.
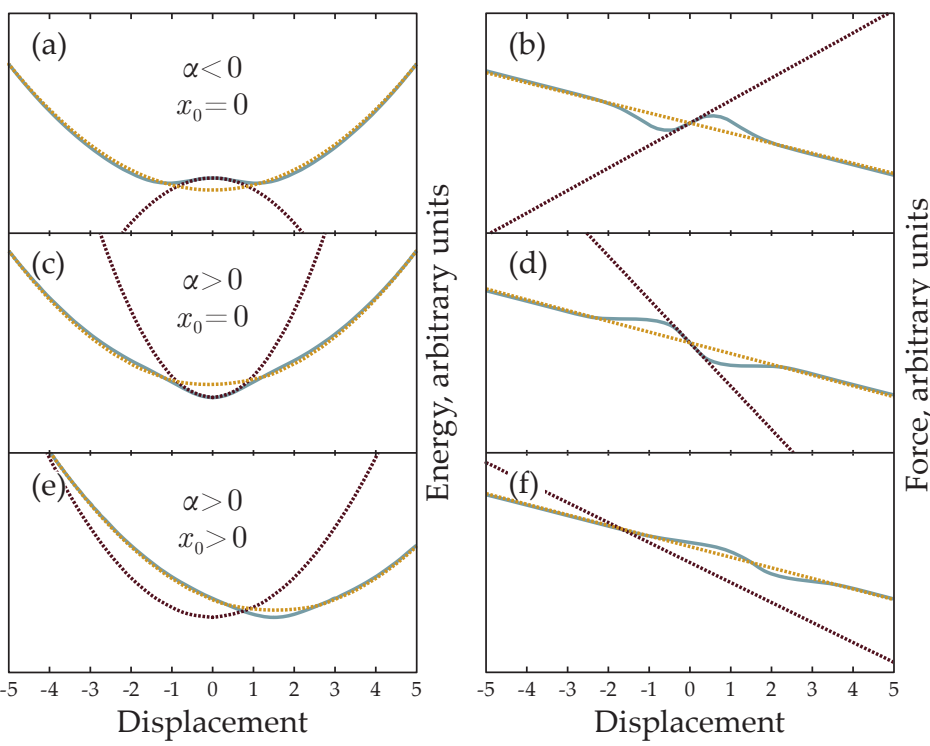

Here the equilibrium position is shifted by the temperature dependent displacement $x_{0}$ from 0 where we assume the equilibrium position at $T=0 K$.

According to harmonic approximation, this interatomic potential can be described (Equation 3.1, 3.4) as,

$$
U(x)=U_{0}+\frac{1}{2} \Phi^{(2)} x^{2} .
$$

And the second-order interatomic force constant can be obtained by

$$
\Phi^{(2)}=\left.\frac{d^{2} U}{\partial x^{2}}\right|_{x=0} .
$$

These equations suggest that if we take the second derivative with respect to atomic position at equilibrium $x=0$ of our fictive interatomic potential (Equation (4.68)) and substitute back in the potential expansion, we should reproduce our fictive $U(x)$. Let consider the second derivative

$$
\frac{d^{2} U}{d x^{2}}=k-2 \alpha \beta e^{-\beta\left(x-x_{0}\right)^{2}}+4 \alpha \beta^{2}\left(x-x_{0}\right)^{2} e^{-\beta\left(x-x_{0}\right)^{2}},
$$

and at the equilibrium position, we get

$$
\Phi^{(2)}=\left.\frac{d^{2} U}{d x^{2}}\right|_{x=0}=k+2 \alpha \beta\left(2 \alpha \beta x_{0}-1\right) e^{-\beta x_{0}^{2}},
$$

substitute this quantity into Equation 4.69, we obtain

$$
U(x)=U_{0}+\frac{1}{2} k x^{2}+2 \alpha \beta\left(2 \alpha \beta x_{0}-1\right) e^{-\beta x_{0}^{2}} x^{2} .
$$




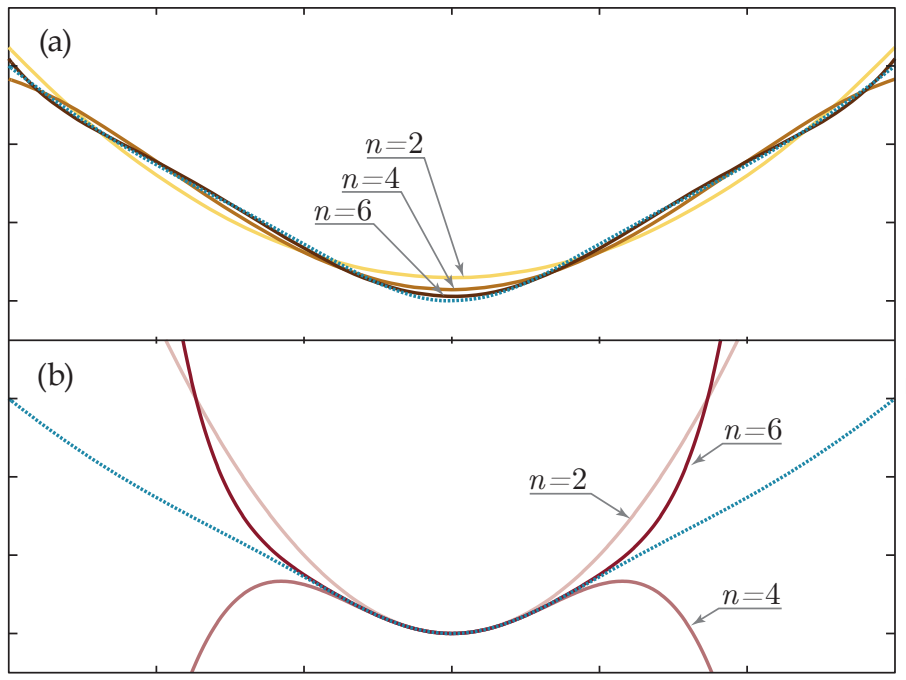

Displacement

Comparing this Equation and the Equation (4.69), it obviously see that the harmonic approximation does not fully reproduce the true potential (see Figure 4.6). This is due to the fact that the harmonic approximation only probed the interatomic potential energy surface at the equilibrium positions, and it totally neglects the fact that there is a shift of the equilibrium from the ideal position at $x=0$ due to temperature effect, here we denote as $x_{0}>0$ (panel (e) in the Figure 4.6). This effect is illustrated in Figure 4.6(a) only the area that close to the equilibrium position is well fitted. Thus this shows the reason why harmonic approximation still work well at low temperature where is not strong vibration of atoms in the crystal. When the effect of temperature comes in the atoms inside the crystal start to move further away from the equilibrium position leading to the uncorrelated between harmonic approximation potential and true interatomic potential (panel (e) in the Figure 4.6).

By changing the equilibrium volume of the crystal as suggested in quasi-harmonic approximation, the indirect correction of temperature effect is somehow added to the harmonic approximation potential; and we consequently expand the probing area in a potential area in the true interatomic potential. This is due to the atomic positions are shifted as the volume of the crystal changes. Hence the results from quasi-harmonic approximation

FIGURE 4.7: The plots show the effects of including higher order terms between the TDEP and harmonic approximation, (a) the case of TDEP exhibits higher order leads to better corrections and (b) the case of harmonic approximation oppositely exhibits with TDEP which is the higher order terms lead to more errors in the fitting. Reprinted figure with permission from ref. [261] (c2013 by the American Physical Society. 
can be used to explain most of the materials thermal properties. But when the effect of anharmonicity is strong. In this case the anharmonicity which is denoted as $\alpha$ changes from positive to negative as shown in Figure 4.6 (a) and (c). The (quasi-) harmonic potential shows totally wrong behavior, resulting in the harmonic approximation predicts the dynamically unstable instead of dynamically stable as it is shown in the true potential (Figure 4.6 (a)). The failure of (quasi-) harmonic approximation leads researchers try to develop a full description of interatomic potential by including the effect of temperature and anharmonicity. First attempt is including more terms in the expansion, ${ }^{39,262,263}$ since the quadratic term cannot explain the phonon-phonon scattering effect that lead to the infinite lattice thermal conductivity. However this did not help to solve the issue as can be seen in Figure 4.7 Thus there were a lot of formalism developed after ${ }^{264-266}$ but there were some issues among these techniques such as can be applied only to the simple system or not suitable for $a b$ initio calculations.

\subsubsection{Temperature Dependent Effective Potential Method (TDEP)}

In this Thesis, I have used the new formalism to obtain an accurate interatomic force constant that invented by Hellman et al. ${ }^{260,261,267}$ so-called Temperature Dependent Effective Potential method (TDEP) to determine the phonon properties. This technique is based on the idea by Born and Hooton ${ }^{262,268}$ that the atoms rarely occupy the positions where the phonon frequencies are negative, instead move into the effective potential of their non-stationary neighbors. This means that if we sampling the potential energy surface at probable atomic position for a given temperature, we should get a harmonic approximation that describes the system at elevated temperatures. According to this suggestion, let us go back to our one-dimensional interatomic potential (Equation (4.68)) and expand our interatomic potential model as a harmonic potential that is centered at $x^{\prime}$

$$
U(x)=\tilde{\Phi}^{(0)}+\frac{1}{2} \tilde{\Phi}^{(2)}\left(x-x^{\prime}\right)^{2},
$$

then perform a molecular dynamics (MD) calculations with an appropriate thermostat that control the temperature of our interest. At the end of MD calculations we obtain a set of $N_{t}$ forces, 
positions and energies, $\left\{F_{t}, x_{t}, E_{t}\right\}$, where $N_{t}$ is the number of timestep. These data can now be used to determine the second order force constants. To do this we have to guess a value for $x^{\prime}$, use the forces from molecular dynamics $\left\{F_{t}\right\}$ and minimize the difference forces between our model potential and BOMD calculated one

$$
\min _{\tilde{\Phi}^{(2)}} \Delta F=\min _{\tilde{\Phi}^{(2)}} \frac{1}{N_{t}} \sum_{t=1}^{N_{t}}\left|F_{t}-\tilde{\Phi}^{(2)}\left(x_{t}-x^{\prime}\right)\right| .
$$

This is easiest realized as a least squares fit of a straight line in forces, as demonstrated in the right panel of Figure 4.6. So the correct second order force constants at the correct equilibrium position $x^{\prime}$ can be obtained by the iterative self-consistent procedure : a guess for $x^{\prime}$ gives us a $\tilde{\Phi}^{(2)}$ and $\Delta F$. This residual force is used to move $x^{\prime}$ to a new position, and new $x^{\prime}$ will go into the loop to find a new a $\tilde{\Phi}^{(2)}$ and $\Delta F$. This iterative process will continue until it finds the correct equilibrium position $x_{0}^{\prime}$ that gives the correct $\Phi^{(2)}$. When the equilibrium position is determined we can safely set any first order term in our model polynomial is zero. The total energies from the MD calculations, $\left\{E_{t}\right\}$ can be used to extract the constant energy term, $\Phi^{(0)}$ from

$$
\Phi^{(0)}=\left\langle E_{t}-\frac{1}{2} \tilde{\Phi}^{(2)}\left(x_{t}-x^{\prime}\right)^{2}\right\rangle_{t} .
$$

This is the best possible potential of the harmonic form at a given temperature as shown in the Figure 4.6. It shows that the true potential and our model potential are perfectly fit at the area further away from equilibrium position and in the case of temperature dependent. Moreover the anharmonic behavior of the potential is implicitly described by the polynomial fit (see Figure 4.6 (a) and (c)). However, it show slightly off at the equilibrium position. This can be solved by increasing more term the polynomial which leads to converges towards the true potential faster than when higher order terms are added to the harmonic approximation see Figure 4.7. Next we will apply this technique to real three-dimensional lattices. 


\subsubsection{The Extension of Temperature Dependent Effective Potential Method to the periodic crystal lattice}

In this section we will describe how to upscale the temperature dependent effective potential method (TDEP) into a real periodic crystal lattice. According to the TDEP, we sampling the potential energy surface at the probable atomic positions giving us a harmonic approximation that describes the system at elevated temperatures. Therefore we can introduce a model Hamiltonian on the harmonic form for $N$ atoms:

$$
\hat{H}=U_{0}+\sum_{i=1}^{N} \frac{\mathbf{p}_{i}^{2}}{2 M_{i}}+\sum_{i=1}^{N} \sum_{j=1}^{N} \mathbf{u}_{i} \overline{\overline{\mathbf{\Phi}}}_{i j} \mathbf{u}_{j} .
$$

Our goal is to determine both the effective second-order interatomic force constants $\overline{\overline{\boldsymbol{\Phi}}}$ matrix and the ground state energy $U_{0}$. The TDEP implementation described in previous Section shows that it is straightforward solve for $\overline{\overline{\mathbf{\Phi}}}$ with all the $(3 N)^{2}$ components of $\overline{\overline{\boldsymbol{\Phi}}}$ as unknown quantities for any diatomic periodic lattice. However, if we implement TDEP to obtain these parameter as is, it would requires many computer resources. Since, the Born-Oppenheimer molecular dynamics already requires a lot of computer resources by itself combining with we need to solve for all components of $\overline{\overline{\mathbf{\Phi}}}$, in order to find all the second-order interatomic force constants will dramatically increase the demand for computer resources. For example the case of diatomic bcc supercell lattice modeled as a $4 \times 4 \times 4$ supercell (128 atoms) has 147456 unknowns in $\overline{\overline{\boldsymbol{\Phi}}}$. To solve all these unknown with supercomputer would be inefficiency.

Luckily, the crystal lattices (Bravais lattices) obey the symmetry, so that many of these unknowns will be equivalent. In case of Bravais lattices, the inversion symmetries are prevailed, thus the permutation of atomic positions and order of the second derivatives do not matter. ${ }^{36}$ This leads to

$$
\begin{aligned}
& \overline{\overline{\mathbf{\Phi}}}^{\alpha \beta}\left(\mathbf{R}_{l}\right)=\overline{\overline{\mathbf{\Phi}}}^{\beta \alpha}\left(-\mathbf{R}_{l}\right) \\
& \Phi_{\mu \nu}^{\alpha \beta}\left(\mathbf{R}_{l}\right)=\Phi_{\nu \mu}^{\alpha \beta}\left(\mathbf{R}_{l}\right) .
\end{aligned}
$$

where $\alpha \beta$ are indices to a unit cell with $N_{u c} \leq N$ atoms and $\mu \nu$ are Cartesian indices. The star vectors $\mathbf{R}_{l}$ is a pair vectors 
in the supercell $\mathbf{R}_{i j}$ connects atoms of type $\alpha$ and $\beta$. Then the interatomic potential energy follows translational invariance in the Bravais lattices, thus the potential energies and forces should remain the same under any arbitrary translation i.e. $U(\mathbf{u}+a)=$ $U(\mathbf{u}) ; \mathbf{F}(\mathbf{u}+a)=\mathbf{F}(\mathbf{u}){ }^{36}$

$$
\sum_{l \alpha} \overline{\overline{\mathbf{\Phi}}}^{\alpha \beta}\left(\mathbf{R}_{l}\right)=0 \text { for each } \beta .
$$

Furthermore, the Bravais lattices also obey the rotation or mirror symmetries, elements of its point group, which leave the molecule invariant. This means that we can related the interatomic force constants matrix $\overline{\overline{\mathbf{\Phi}}}$ with the symmetry operation, $\hat{S}$. The components of $\overline{\bar{\Phi}}$ are related as follows ${ }^{269}$ :

$$
\Phi_{i j}^{\alpha \beta}\left(\mathbf{R}_{l}\right)=\sum_{\mu \nu} \Phi_{k l}^{\mu \nu}\left(\mathbf{R}_{l}\right) S^{\mu \alpha} S^{\nu \beta},
$$

In order to utilize these symmetries properties to reduce the unknowns, $\theta_{k}$, we operate as following: first we span our interatomic force constants matrix tensor, $\overline{\overline{\mathbf{\Phi}}}$ that related to $\Phi_{\mu \nu}^{\alpha \beta}\left(\mathbf{R}_{l}\right)$ with unknowns variable, called $\theta_{k}$. This $\theta_{k}$ will run from 1 until it reaches all vectors $\mathbf{R}_{l}$ that are determined by the size of the supercell. We will call the maximum $\mathbf{R}_{l}$ as a cutoff radius $\mathbf{R}_{\text {cutoff. Then }}$ we applied all the symmetries properties (Equation 4.78-4.81) to construct the matrix tensor $\overline{\overline{\mathbf{C}}}$ from a set of displacements $\mathbf{U}$ that relates to the symmetry constrains of our interesting system. This $\overline{\overline{\mathbf{C}}}$ will be span to all the displacements $\mathbf{U}$ within $\mathbf{R}_{\text {cutoff }}$ After obtaining $\overline{\overline{\mathbf{C}}}$, we use it to determine which of these unknowns $\theta_{k}$ are equivalent to each other, or 0 by symmetry and leave only the inequivalent $\theta_{k}$. The amount of inequivalent $\theta_{k}$ are depended on our interested lattice symmetry. For our example diatomic bcc lattice, we can reduce the $\theta_{k}$ from 147456 unknowns into 11 unknowns of $\theta_{k}$.

The set of inequivalent $\theta_{k}$ will form a matrix $\Theta$, and uses it to construct our model harmonic forces, $\mathbf{F}_{\mathrm{H}}$ that relates to the model Hamiltonian (Equation 4.77) as

$$
\mathbf{F}_{\mathrm{H}}=\overline{\overline{\mathbf{C}}}(\mathbf{U}) \boldsymbol{\Theta},
$$

where the component of these forces matrix tensor can be expressed as

$$
f_{i}=\sum_{k} \theta_{k} \sum_{\delta} c_{k \delta}^{i} u_{\delta}
$$


where the coefficients $c_{k \delta}^{i}$ is the coefficients for linear combinations of all displacement components $u_{\delta}$ within $\mathbf{R}_{\text {cutoff }}$ after symmetry operations are applied to a given lattice points and the matrix tensor $\Theta$ will hold all the inequivalent $\theta_{k}$. Here we perform Born-Oppenheimer molecular dynamics calculations with an appropriate thermostat controlling the temperature to obtain a set of displacements $\left\{\mathbf{U}_{t}^{\mathrm{MD}}\right\}$, forces $\left\{\mathbf{F}_{t}^{\mathrm{MD}}\right\}$ and potential energies $\left\{E_{t}^{\mathrm{MD}}\right\}$ where the subscript $t$ is denotes the displacements, forces and energies at timestep $t$. Then we minimize the difference between the forces from BOMD and the model harmonic forces (Equation 4.82) from our model Hamiltonian that are given by

$$
\begin{aligned}
\min _{\Theta} \Delta \mathbf{F} & =\min _{\Theta} \frac{1}{N_{t}} \sum_{t=1}^{N_{t}}\left|\mathbf{F}_{t}^{\mathrm{MD}}-\mathbf{F}_{t}^{\mathrm{H}}\right|^{2}= \\
& =\min _{\Theta} \frac{1}{N_{t}} \sum_{t=1}^{N_{t}}\left|\mathbf{F}_{t}^{\mathrm{MD}}-\overline{\overline{\mathbf{C}}}\left(\mathbf{U}_{t}^{\mathrm{MD}}\right) \boldsymbol{\Theta}\right|^{2}= \\
& =\min _{\Theta} \frac{1}{N_{t}}\left\|\left(\begin{array}{c}
\mathbf{F}_{1}^{\mathrm{MD}} \\
\vdots \\
\mathbf{F}_{N_{t}}^{\mathrm{MD}}
\end{array}\right)-\left(\begin{array}{c}
\overline{\overline{\mathbf{C}}}\left(\mathbf{U}_{1}^{\mathrm{MD}}\right) \\
\vdots \\
\overline{\overline{\mathbf{C}}}\left(\mathbf{U}_{N_{t}}^{\mathrm{MD}}\right)
\end{array}\right) \boldsymbol{\Theta}\right\| .
\end{aligned}
$$

where, the $N_{t}$ is the total number of timesteps in the molecular dynamics calculations. The linear least squares solution $\Theta$ can be obtained by the pseudo inverse method likes a Moore-Penrose pseudo inverse

$$
\boldsymbol{\Theta}=\left(\begin{array}{c}
\overline{\overline{\mathbf{C}}}\left(\mathbf{U}_{1}^{\mathrm{MD}}\right) \\
\vdots \\
\overline{\overline{\mathbf{C}}}\left(\mathbf{U}_{N_{t}}^{\mathrm{MD}}\right)
\end{array}\right)^{+}\left(\begin{array}{c}
\mathbf{F}_{1}^{\mathrm{MD}} \\
\vdots \\
\mathbf{F}_{N_{t}}^{\mathrm{MD}}
\end{array}\right) .
$$

then the solutions are then mapped to the force constant matrix $\Phi_{\mu \nu}^{\alpha \beta}\left(\mathbf{R}_{l}\right)$,

$$
\Theta \longrightarrow \Phi_{\mu \nu}^{\alpha \beta}\left(\mathbf{R}_{l}\right),
$$

where the matrix tensor dimensions can be expressed, the force vector $\left(\mathbf{F}_{t}^{\mathrm{MD}}\right)$ will have $3 N N_{t}$ rows. Each submatrix tensor $(\overline{\overline{\mathbf{C}}})$ will have $3 N$ rows and $N_{\theta}$ columns. The full coefficient matrix $\left(\mathbf{U}_{t}^{\mathrm{MD}}\right.$ ) has $3 N N_{t}$ rows and $N_{\theta}$ columns. $\Theta$ is a row vector with $N_{\theta}$ rows. This symmetry implementations can help us to improve the he numerical accuracy and computation efficiency when comparing to another method. Since we use the symmetries to eliminate the unnecessary interatomic force constants beforehand. So that if there is the numerical errors appear during force calculation should average or cancel them out leads to more reliable 
results. Unlike another method that use symmetries to fasten the calculations time by determining the interaction in one direction and then using the symmetry relations to translate and rotate that interaction. If there is unavoidable numerical errors in the first calculation, they will propagate to all interactions leads to poor accuracy.

From the force constant matrix $\Phi_{\mu \nu}^{\alpha \beta}\left(\mathbf{R}_{l}\right)$, we can now solve for all the components to find dynamical matrix $\overline{\overline{\mathbf{\Phi}}}(\mathbf{q})$ via Equation (3.13) and the number of coordination shells which is related to $\mathbf{R}_{l}$. After obtaining $\overline{\overline{\mathbf{\Phi}}}(\mathbf{q})$, we now can proceed to calculate phonon dispersion relations from the eigenvalue equation (3.11) and thermal properties. The full procedure and detail on how to implement TDEP by Born-Oppenheimer molecular dynamics can be found on ref. [260, 261, 270]. In next section, we looks into how to obtain free energy and lattice thermal conductivity from BOMD calculations with TDEP.

\subsubsection{Free energy calculations}

Following discussion is the method of determining the free energy for temperature dependent effective potential. First we begin with write down the full Helmholtz free energy,

$$
F=\left\langle E_{k}\right\rangle+\left\langle U_{\mathrm{BOMD}}\right\rangle+F_{\mathrm{vib}}+F_{\mathrm{AH}},
$$

where $\left\langle E_{k}\right\rangle$ the average kinetic energy, $U_{\mathrm{BOMD}}$ is the temperature dependent potential energy, $F_{\text {vib }}$ is the harmonic Helmholtz free energy , $F_{\mathrm{AH}}$ is the anharmonic Helmholtz energy. Here the first and third term is quite easily determined. The $\left\langle E_{k}\right\rangle$ can directly be obtain normal Born-Oppenheimer molecular dynamics calculations with the thermostat to regulate temperature. And the $F_{\text {vib }}$ can be obtained from Equation (3.24) in the Section (3.1) by using the phonon frequencies that obtain from temperature dependent effective potential method (TDEP) which also includes the zero point energy.

Next we need to determine temperature dependent potential energy, $U_{\text {BOMD }}$. The $U_{\text {BOMD }}$ can directly be obtained via BOMD calculations. But the problem is that $U_{\mathrm{BOMD}}$ is rapidly oscillating over time due to the numerical fluctuation, thus it requires long calculation time to converge. To get around this issue, we use 
the fact that the model Hamiltonian potential energy from TDEP should, on average, be equal to potential energy from BOMD. If this is true, it means that the full anharmonicity is included in the $\left\langle U_{\mathrm{BOMD}}\right\rangle$. According to this concept, let us consider the model Hamiltonian potential energy in TDEP which is written as

$$
\begin{aligned}
U_{\mathrm{HTEP}}(t) & =U_{0}+\frac{1}{2} \sum_{i j \alpha \beta} \Phi_{i j}^{\alpha \beta} u_{i}^{\alpha}(t) u_{j}^{\beta}(t) \\
U_{0} & =U_{\mathrm{HTEP}}(t)-\frac{1}{2} \sum_{i j \alpha \beta} \Phi_{i j}^{\alpha \beta} u_{i}^{\alpha}(t) u_{j}^{\beta}(t) .
\end{aligned}
$$

Since $\left\langle U_{\mathrm{BOMD}}\right\rangle=\left\langle U_{\mathrm{HTEP}}\right\rangle$, we should obtain

$$
U_{0}=\left\langle U_{\mathrm{BOMD}}(t)-\sum_{i j \alpha \beta} \frac{1}{2} \Phi_{i j}^{\alpha \beta} u_{i}^{\alpha}(t) u_{j}^{\beta}(t)\right\rangle .
$$

Here, the harmonic thermal excitations are removed from the BOMD potential energies. Consequently, the accuracy of $U_{0}$ is improved by roughly an order of magnitude. In addition, it showed in the Section (4.8.1) that if we include higher order terms in the potential energy expansion, it leads to better corrections. This result will further serve to minimize these fluctuations.

At this point, we have obtain potential energy from BOMD calculations, $U_{\text {BOMD }}$ and model Hamiltonian potential with TDEP, $U_{\text {HTEP. }}$ We can use these two potential energies to determine the anharmonic correction of free energy as the reference point by thermodynamic integration method that was discussed in Section (4.7.5). So the full free energy will be

$$
F=\left\langle E_{k}\right\rangle+U_{0}+F_{\mathrm{vib}}+\underbrace{\int_{0}^{1}\left\langle\left. U_{\mathrm{BOMD}}\right|_{\lambda}-\left.U_{\mathrm{HTEP}}\right|_{\lambda}\right\rangle_{t} d \lambda}_{\Delta F_{\mathrm{AH}}} .
$$

Since the $U_{\text {HTEP }}$ is developed to describe the anharmonic system with harmonic form as good as possible. After integrate over the Kirkwood coupling parameter $\lambda$, the different between $U_{\text {BOMD }}$ and $U_{\text {HTEP }}$ would be small leading to anharmonic correction term $\Delta F_{\mathrm{AH}}$ is small. As discussed, the thermodynamic integration technique is numerically inefficient. Because the system size effect, even though we can accurately control the numerics. ${ }^{271}$ As shown by Hellman et al. ${ }^{270}$ that we can get a reasonable free energy when the system size above 100 atoms with the free energy is converged within $1 \mathrm{meV} /$ atom. After obtaining the free energy, we 
can use to calculate the constant heat capacity $C_{p}=-T \frac{\partial^{2} G}{\partial T^{2}}$ and the volume Grüneise parameters $\gamma_{\lambda}=\left(\frac{-V}{\omega_{\lambda}}\right)\left(\frac{\partial \omega_{\lambda}}{\partial V}\right)$, where the Gibbs free energy can be mapped out from Helmholtz free energy from Gibbs-Helmholtz relation $G=-V^{2}\left[\frac{\partial(F / V)}{\partial V}\right]$ at constant temperature, $\lambda$ denotes the phonon mode with wave vector $\mathbf{q}$ and branch index $s$ at each temperature.

\subsubsection{Lattice thermal conductivity calculations}

To calculate the lattice thermal conductivity, the model potential energy (Equation (4.77)) need to expand to the third term with the third-order interatomic force constant, $\Phi^{(3)}$. Since the quadratic potential energy cannot be used to obtain a corrected lattice thermal conductivity due to lack of three phonons scattering. Without phonon scattering event, the phonons will live forever leading to a infinite lattice thermal conductivity. The new model Hamiltonian can be written as ${ }^{267}$

$$
\begin{aligned}
H= & U_{0}+\sum_{i} \frac{\mathbf{p}_{i}^{2}}{2 m_{i}}+\frac{1}{2 !} \sum_{i j \alpha \beta} \Phi_{i j}^{\alpha \beta} u_{i}^{\alpha} u_{j}^{\beta}+ \\
& \frac{1}{3 !} \sum_{i j k \alpha \beta \gamma} \Phi_{i j k}^{\alpha \beta \gamma} u_{i}^{\alpha} u_{j}^{\beta} u_{k}^{\gamma},
\end{aligned}
$$

where $m_{i}, \mathbf{p}_{i}$, and $u_{i}$ are the mass, momentum, and displacements of atom $i, \Phi_{i j}^{\alpha \beta}$ is the second-order force constant $\Phi^{(2)}$ that couple for each pair of atoms $i$ and $j$, and $\Phi_{i j k}^{\alpha \beta \gamma}$ is the third-order force constant $\Phi^{(3)}$ that couple for each triplet of atoms $i, j$, and $k$ where $\alpha \beta \gamma$ are the Cartesian indices. Although there is an additional third-order term, the TDEP is still possible to apply to find the $\Phi^{(3)}$ in the similar manner as $\Phi^{(2)}$ that we introduced at earlier section. ${ }^{270}$ After performing Born-Oppenheimer molecular dynamics calculations, the $\Phi^{(2)}$ and $\Phi^{3}$ are extracted and mapped into the dynamical matrix $\overline{\overline{\mathbf{\Phi}}}(\mathbf{q})$ according to the number of coordination shells, $\mathbf{R}_{l}$ (the maximum of $\mathbf{R}_{l}$ is determined by the supercell size). Then the phonon dispersion relation can be obtained from the eigenvalue equation (3.11). After obtaining the phonon dispersion relation, we use the phonon Boltzmann transport equation to obtain the phonon current $J_{\alpha}$ density in the $\alpha$ direction through the sample when the temperature gradient $\Delta T$ is applied. Here we use the linearized collision approximation to solve phonon Boltzmann transport equation. Let start with the 
heat current density,

$$
J_{\alpha}=\frac{1}{V} \sum_{\mathbf{q} s} \hbar \omega_{\mathbf{q} s} \mathrm{v}_{\mathbf{q} s \alpha} n_{\mathbf{q} s \alpha},
$$

where $V$ is the crystal volume, $\mathbf{v}_{\mathbf{q} s \alpha}$ is the phonon velocity in the mode $\mathbf{q}$ and branch (polarization) index $s$, and if the applied temperature gradient is small, $n_{\mathbf{q} s \alpha}$ is the non equilibrium distribution functions which can be linearized as

$$
n_{\mathbf{q} s \alpha} \approx n_{\mathbf{q} s}^{0}-\mathbf{v}_{\mathbf{q} s \alpha} \tau_{\mathbf{q} s \alpha} \frac{d n_{\mathbf{q} s}^{0}}{d T} \frac{d T}{d \alpha},
$$

where $n_{\mathbf{q} s}^{0}$ is the equilibrium (Bose) distributions at temperature $T, \alpha$ is denoted a Cartesian direction and $\tau_{\mathbf{q} s \alpha}$ is the phonon lifetime in the transport direction. From this linearization of non equilibrium distribution functions, substituting back to the $J_{\alpha}$, we get

$$
\begin{aligned}
J_{\alpha} & =\frac{1}{V} \sum_{\mathbf{q} s} \hbar \omega_{\mathbf{q} s} \mathrm{v}_{\mathbf{q} s \alpha}\left(n_{\mathbf{q} s}^{0}-\mathrm{v}_{\mathbf{q} s \alpha} \tau_{\mathbf{q} s \alpha} \frac{d n_{\mathbf{q} s}^{0}}{d T} \frac{d T}{d \alpha}\right) \\
& =\frac{1}{V} \sum_{\mathbf{q} s} \hbar \omega_{\mathbf{q} s} \mathrm{v}_{\mathbf{q} s \alpha} n_{\mathbf{q} s}^{0}-\sum_{\mathbf{q} s} \hbar \omega_{\mathbf{q} s} \mathrm{v}_{\mathbf{q} s \alpha}^{2} \tau_{\mathbf{q} s \alpha} \frac{d n_{\mathbf{q} s}^{0}}{d T} \frac{d T}{d \alpha} \\
& =\frac{1}{V} \sum_{\mathbf{q} s} \hbar \omega_{\mathbf{q} s} \mathrm{v}_{\mathbf{q} s \alpha} n_{\mathbf{q} s}^{0}-\kappa_{\alpha} \frac{d T}{d \alpha} .
\end{aligned}
$$

Comparing with the heat flux density

$$
J_{\alpha}=-\kappa_{\alpha} \Delta T
$$

Here, the first term $\hbar \omega_{\mathbf{q} s} \mathrm{v}_{\mathbf{q} s \alpha} n_{\mathbf{q} s}^{0}$ should vanish since there is no heat conduction at equilibrium. The $\kappa_{\alpha}$ is defined as lattice thermal conductivity that is transported in $\alpha$ direction which is given by

$$
\kappa_{\alpha}=\frac{1}{V} \sum_{\mathbf{q} s} C_{\mathbf{q} s} \mathbf{v}_{\mathbf{q} s \alpha}^{2} \tau_{\mathbf{q} s \alpha}
$$

where $C_{\mathbf{q} s}=\hbar \omega_{\mathbf{q} s} \frac{\partial n_{\mathbf{q} s}^{0}}{\partial T}$ is the mode specific heat. The phonon lifetimes are determined from the linearized phonon Boltzmann equations (PBE),

$$
\begin{aligned}
k_{\mathrm{B}} T \mathrm{v}_{\lambda \alpha} \frac{\partial T}{\partial x_{\alpha}} \frac{\partial n_{\lambda \alpha}^{0}}{\partial T}= & \sum_{\lambda^{\prime} \lambda^{\prime \prime}}\left[W_{\lambda \lambda^{\prime} \lambda^{\prime \prime}}^{+}\left(F_{\lambda^{\prime \prime}}-F_{\lambda^{\prime}}-F_{\lambda}\right)\right. \\
& \left.+\frac{1}{2} W_{\lambda \lambda^{\prime} \lambda^{\prime \prime}}^{-}\left(F_{\lambda^{\prime \prime}}+F_{\lambda^{\prime}}-F_{\lambda}\right)\right] \\
& +\sum_{\lambda^{\prime}}\left(W_{\lambda \lambda^{\prime}}^{i m p}\left(F_{\lambda^{\prime}}-F_{\lambda}\right)\right),
\end{aligned}
$$


where the left-hand side of this equation describes the phonon diffusion induced by the thermal gradient and the equilibrium phonon distribution. On the right-hand side represents the collision events that relate to the 3-phonon scattering matrices $\left(W_{\lambda \lambda^{\prime} \lambda^{\prime \prime}}^{ \pm}\right)$where $W_{\lambda \lambda^{\prime} \lambda^{\prime \prime}}^{ \pm}$is depended on the component in $\overline{\overline{\mathbf{\Phi}}}$ (q) and impurity (mainly isotope) scattering matrix ( $\left.W_{\lambda \lambda^{\prime}}^{i m p}\right)$. The notation $\lambda$ denotes the phonon mode of wave vector $\mathbf{q}$ and branch index $s$. The full iterative solution of the phonon Boltzmann equations (PBE) that was introduced by Omini and Sparavigna ${ }^{272}$ and implemented by Ward et al. ${ }^{273}$ In this method the phonon lifetime is related through

$$
\tau_{\mathbf{q} s \alpha}=\frac{T F_{\mathbf{q} s \alpha}}{\hbar \omega_{\mathbf{q} s} v_{\mathbf{q} s}}
$$

where $F_{\mathbf{q} s \alpha}$ is the iteration functions that relates to the phonon scattering rates and $\omega_{\mathbf{q} s}$ is the phonon frequencies. To get phonon lifetimes, the PBE is solved on the odd three dimensions $\mathbf{q}$ mesh where the momentum conservation is fulfilled. For the energy conservation, there are two computational methods that can be used : (i) The adaptive broadening scheme of Yates et al. ${ }^{274}$ or (ii) a tetrahedron approach. ${ }^{223,275,276}$ Both methods should converge to the same results. But the criteria of choosing between these two methods are tetrahedron approach is computationally efficient for the system with small numbers of atoms per unit cells, while the materials with large unit cells, the adaptive Gaussian scheme was preferable since it more easily handled the many brach crossing. After obtaining the phonon lifetimes, the lattice thermal conductivity can be calculated. Sometime, the materials have microstructure with average grain size, $l$. The lattice thermal conductivity accumulation (or lattice thermal conductivity with grain boundary scattering) can be determined ${ }^{267}$ by

$$
\kappa_{\alpha \alpha}(l)=\frac{1}{V} \sum_{\mathbf{q} s} C_{\mathbf{q} s} v_{\mathbf{q} s}^{2} \tau_{\alpha \mathbf{q} s} \Theta\left(l-\mid \mathbf{v}_{\mathbf{q} s} \tau_{\alpha \mathbf{q} s}\right),
$$

where $\Theta(x)$ is the Heaviside step function. This is the end of theoretical methods discussion. In the next chapter, we will consider the material synthesis technique that was used in my study. 



\title{
Chapter 5
}

\section{Deposition processes}

\author{
"Method is much, technique is much, but inspira- \\ tion is even more." \\ Benjamin N. Cardozo, former Associate Justice \\ of the Supreme Court of the United States
}

In vapor deposition of thin films, ${ }^{277}$ the films are grown by combination of "building blocks" of free atoms and molecules in vapor phase which condense into solid phase and deposit on a surface as film. There are two main approaches: physical vapor deposition (PVD) and chemical vapor deposition (CVD). In chemical vapor deposition, the films are grown on the substrate (the object we need to coat) by chemically reacting gases. For physical vapor deposition, the vapor phase originates from a solid or liquid which is transformed into vapor by electricity, electron beam, arc, or laser or sputtered. In this chapter, I will describe sputter deposition, which I have used to synthesize material in this Thesis.

\subsection{The physics of sputtering}

Sputtering was first studied by W. R. Grove who found thin metal layer deposition on glass tube when he studied a gas discharge in a glass tube. ${ }^{278}$ "Sputtering" is a physical phenomenon which ejects particles of material from a solid surface (known as target). The impact of energetic ions (often $\mathrm{Ar}^{+}$) which is generated inside plasma can eject or "sputter" the particles ${ }^{\mathrm{I}}$ from target by transferring momentum and energy between ions and atoms at the target. The sputtered particles are transported to the substrate where they condense and form a film. A schematic sputtering setup is shown in Figure 5.1(a). This sputtering setup is called diode sputtering. A base pressure between high to ultra-high vacuum $\left(\sim 10^{-7}-10^{-9}\right.$ Torr) inside the chamber is often required to avoid contamination in films.

(I) These sputtered particle are usually of 1-2 atoms but may be a clusters of atoms. 
FIGURE 5.1: (a) Schematic drawing of sputtering setup. (b) Sputtering process at surface of target.
(II) Radio frequency ac power (RF diode plasma) or pulse DC power (pulse-DC diode plasma) can also be applied to ignite plasma as well.
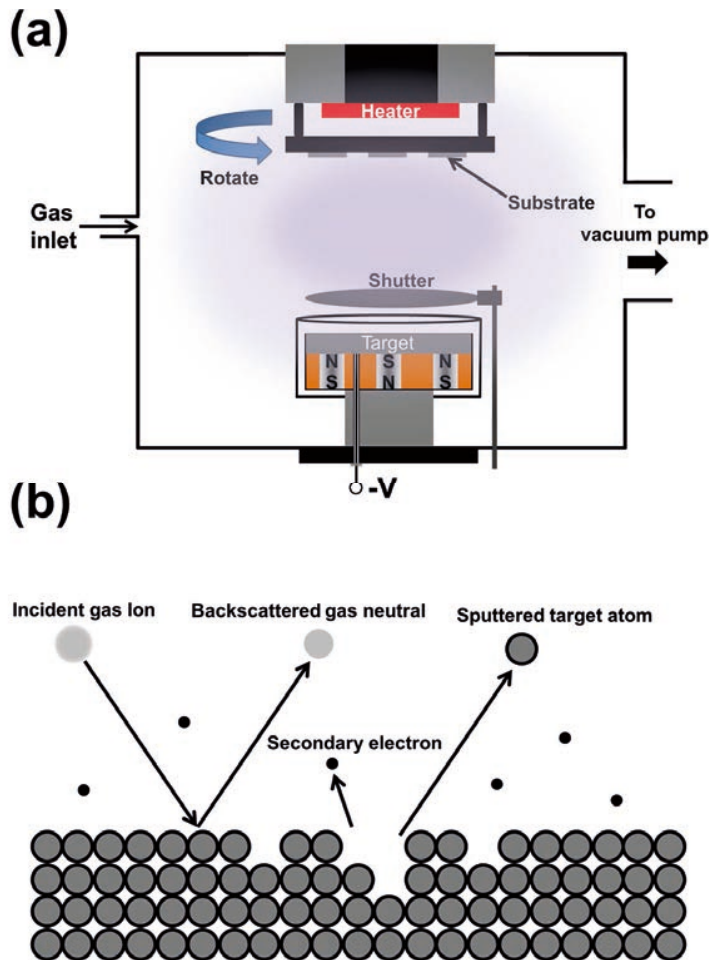

\subsubsection{Plasma}

Plasma is essential for the sputtering process. The word "plasma" was conceived by Irving Langmuir in 1928, ${ }^{279}$ when he studied the oscillating behavior of ionized gases in high current vacuum tubes. A definition of plasma ${ }^{279}$ is :

Plasma is a weakly ionized gas, including a collection electrons, ions, and neutral atomic which is when considering averaged charges over all particles in plasma volume is electrically neutral.

Plasma mainly categorizes into two types of processing discharges, low gas pressure discharge and high gas pressure discharge. In sputtering process, a low gas pressure discharge plasma (glow discharge plasma) which is a discharge of 0.001-1 Torr (0.13-133 $\mathrm{Pa}$ ) of argon (Ar) or other noble gases is used. To ignite plasmas, high DC voltage (several $\mathrm{kV}$ ) is applied by power supply between metal electrodes (called DC diode plasma) ${ }^{\mathrm{II}}$. The mechanism which turns a gas to plasma starts when an electron is 
ejected from cathode given an initial current $I$. That electron is accelerated to the anode by the electric field (E) with energy $(U=-e V)$. As the voltage increases, electrons will gain energy enough when they pass through or collide with neutral gas atoms and ionize them. The collision is followed by the electron impact ionization $^{\text {III }}$

$$
e^{-}+A r^{0} \longrightarrow A r^{+}+2 e^{-}
$$

where $\mathrm{Ar}$ is a argon neutral atom, $\mathrm{Ar}^{+}$is a argon positive ion, and $e^{-}$is an electron. As results of the process, there are two electrons that will collide with another neutral gas. The ions are drifted by electric field and hit the cathode where electrons (often called secondary electrons) will be ejected from cathode. This is a first stage of low-pressure plasma discharge regime which is called Townsend discharge. The characteristic of this regime is a very low flow of current due to a small amount of charge carriers in system. The current in the Townsend discharge will increase rapidly due to continued emission of electrons from ionization process and ions striking the cathode, but the voltage is limited by constant impedance of power supply. This process will continue until an avalanche of ionization collisions occurs. The voltage will drop with a sharp rise of current since the gas in the system breaks down into ions, so that the system will have enough secondary electrons to produce a number of ions that regenerate the same amount of electrons. Therefore, the plasma becomes self-sustaining and begins to glow due to de-excitation process in plasma allows light emission from plasma. This regime is called the normal glow discharge. Here, if one raises the power applied to the electrodes, ions will bombard the entire cathode surface. Further increase in power will lead to increased current density and voltage, the abnormal glow discharge regime. This regime is the one used in plasma processing e.g. sputtering, plasma etching, and plasma enhanced CVD. If current continues to rise, the cathode will be heated. Consequently, thermionic electrons are emitted from cathode surpassing secondary electrons leading to a second avalanche. The voltage drops rapidly with high current allow arc propagate toward cathode. Arcs are defined as gas or vapor discharges where the cathode voltage drops in order to minimize ionization. Arcs can be self-sustained with high current by providing electron emission from cathode and anode. This regime is known as arc discharge and is mainly used in cathodic arc deposition process.
(III) The other reactions in plasma can be found in $\mathrm{M}$. Ohring. ${ }^{277}$ 


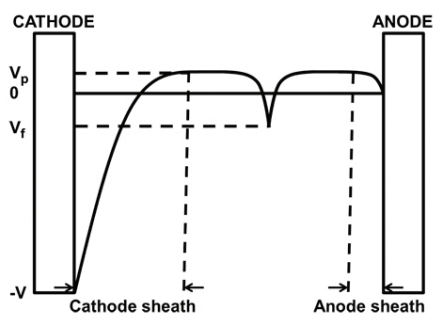

FIGURE 5.2: The potential distribution from DC glow discharge. Typically in normal sputter system, the cathode is biased with negative voltage. Anode is a chamber wall including substrate which is grounded. $V_{p}$ is the plasma potential and dot lines indicate region of sheath. Adapted from M. Ohring. 277

\subsubsection{The plasma, floating, and bias potentials, potential distribution, and sheath}

If any object is inserted into the plasma, its potential will be slightly negative with respect to the plasma unless it is grounded $(V=0)$. Because of the dissimilarity between electrons and ions (i.e., in mass, velocity, and energy), electrons have higher mobility than ions. ${ }^{280}$ Thus electrons can accumulate on surface of object (e.g., substrate, chamber wall, etc.) giving a negative potential (floating potential, $V_{f}$ ). Since electrons accumulate on the surface, they leave positive ions behind meaning that plasma will have positive potential (plasma potential, $V_{p}$ ) relative to floating potential (and relative to ground). Therefore, each object will develop a depletion sheath which is called dark space, because of the electron inside plasma is repelled by floating and grounded potential, so that in the area close to electrode or immersed object will have low electron density result in less ionization process (low plasma density) yielding less emissions of light. In DC sputtering process, there are two major dark spaces which are cathode and anode sheaths. Since cathode (target) is connected to negative voltage and generate sputtered species and anode (chamber wall) is grounded. The potential distribution in typical sputtering process is shown in Figure 5.2 By application of a negative voltage or bias potential, the floating potential is replaced. This method is used to attract ions to bombard substrate which can be used to improve film quality. In contrast, if positive bias potential is applied to a substrate, it will draw a lot of electrons yielding substrate heating and non-uniform current density contribution. Positive bias should thus usually be avoided.

\subsubsection{Transport of sputtered species}

After the sputtering event occurs, the sputtered species from surface of the target are transported into the plasma. However, the sputtered species may lose their energy by collision with sputtering gas and charged particles in plasma. Therefore, many of the sputtered species can be lost during transport. Only some of them will arrive and condense on the substrate. We can define transportation regime of sputtered species into three regimes due to the mean free path of sputtered particles $\lambda_{\mathrm{mfp}}$ which depends on pressure of sputtering gas (number of sputtering gas atoms and 
volume of the chamber) and size of sputtering particles in the system, and temperature. ${ }^{277,281}$ For the case of two gases, the mean free path of gas a in gas $\mathrm{b}, \lambda_{\mathrm{a}, \mathrm{b}}$ is given ${ }^{282}$ by

$$
\lambda_{\mathrm{a}, \mathrm{b}}=\frac{4 k_{\mathrm{B}} T}{\pi\left(d_{\mathrm{a}}+d_{\mathrm{b}}\right)^{2} P_{2}\left(1+\frac{M_{\mathrm{a}}}{M_{\mathrm{b}}}\right)^{1 / 2}},
$$

where $k_{\mathrm{B}}$ is the Boltzmann's constant, $d_{\mathrm{a}}$ and $d_{\mathrm{b}}$ are the molecular diameter of gas a and gas $\mathrm{b}, m_{\mathrm{a}}$ and $m_{\mathrm{b}}$ are the mass of gas a and $\mathrm{b}, P_{\mathrm{b}}$ is the partial pressure of gas $\mathrm{b}$, and $T$ is the absolute temperature. First, ballistic regime is a regime that sputtered species move straight from the target without collisions giving sputtered species on the substrate surface have high energy. The films that are grown under this condition will have good stoichiometry and high density. Second, diffusive regime is a regime that sputtered species have low mean free path meaning they will lose all of their energy due to collision and the motion of species is random which is why this regime is also called the thermalized regime. The transition regime is located between first two regimes where sputtered atoms suffer collisions but still retain some energy. It is therefore better to run sputtering in ballistic regime. However, this requires low pressure and we have to face the problem that plasma cannot sustain itself due to low sputtering gas. Therefore, the transition regime is usually the best choice to run sputtering process with working pressure of 3-6 mTorr (0.4-0.8 Pa) and the distance from material to substrate is approximately $10 \mathrm{~cm}$.

\subsubsection{Effects at the substrate}

When sputtered species arrive at the substrate, they will condense on surface. This is determined by the sticking coefficient. The sticking coefficient affects the film composition and deposition rate because of its temperature dependence. During thin film deposition, the substrate can be heated. This will give extra energy to the sputtered species (adatom) which can move on the substrate surface therefore, we can obtain dense and well oriented film. On the other hand, this extra heat will allow sputtered species to evaporate from substrates yielding off-stoichiometric films. This is important point when one needs to grow film which includes high vapor pressure material. Resputtering is another process that causes films to lose the intended stoichiometry. Resputtering is a process in which deposited species on the substrate 
are resputtered by highly energetic ions (closely to the target voltage, i.e., $\sim 300 \mathrm{eV}$ ) from the plasma or neutrals backscattered from target. This situation is easy to observe when we apply high bias voltage to substrate. This high bias voltage can provide denser film since it will induce ions to bombard the growing film. However, if the bias voltage is too high, they will result in high energetic ions can sputter the growing film. This resputtering process strongly affects composition and microstructure, especially films consisting of both light and heavy elements.

\subsection{Type of sputtering process}

There are many modes of sputtering e.g., the use of reactive gases during process (known as reactive sputtering), the use of magnets behind target, called magnetron sputtering and different applied voltage forms e.g. direct current (DC magnetron sputtering), radio frequency (RF magnetron sputtering), or high-power impulse (High-Power Impulse Magnetron Sputtering, HiPIMS). These many forms of sputtering technique have been widely used for coating in both research work and industrially. In this thesis, I use reactive DC magnetron sputtering to grow scandium nitride thin films.

\subsubsection{DC magnetron sputtering}

Considering the ionization process, Equation 5.1, shows that we need sufficient amount of electrons to maintain plasma discharge. Therefore a high voltage and high gas pressure are required to ignite the plasma. This makes all sputtered species are thermalized due to collision as we discussed in Section (5.1.3). This leads to low deposition rate with poor film quality due to low energy adatom and impurity incorporation. One could guess these requirements are not appropriate for industrial appreciation of sputtering process. Thus Penning showed that by the use of electric and magnetic field, we can trap outgoing secondary electrons close to the target resulting in increased ionization of the plasma near the surface of the target which enhancing the sputtering efficiency. ${ }^{277,281,283}$ The main concept behind this method is the interaction of charged particles with electric and magnetic 
fields as given by the Lorentz force

$$
\mathbf{F}=m \frac{d \mathbf{v}}{d t}=e(\mathbf{E}+\mathbf{v} \times \mathbf{B})
$$

where $e$ is the electronic charge, $m$ is the particle mass, here is electron mass, $\mathbf{v}$ is the velocity, $E$ the electric field and $B$ the magnetic field. If the vector product $\mathbf{E} \times \mathbf{B}$ is not equal to zero, this produces a circular drift electron motion. To implement this idea, the magnetron was invented. Figure 5.3 shows schematic drawing of magnetron setup, the permanent magnets are placed under the target. Thus a magnetron applies a static magnetic field located parallel to the target surface. The negative voltage applied to target (cathode) will generated electric field, electrons will experience the effect of electric field causing their motion in loop form parallel to the cathode surface along magnetic field. For this purpose, the secondary electrons, which are produced during ion bombardment (see Figure 5.1(a)) can be confined by the field from the magnetron. Therefore, the advantage of inserting magnets under target allows sputtering processes to operate at low pressure (about $m$ Torr) within a few hundred volts of applied voltage. In addition, the confinement of the plasma close to target will increase sputtering rate or deposition rate as shown in Figure 5.3. Therefore, this technique is used in both research and industrial production, since it has much higher deposition rate (can be 10 times higher) than diode sputtering. There are two standard types of magnetron configurations, balanced magnetron or unbalanced magnetron type I or type II. For a balanced magnetron, the strength of inner and outer magnets is equal leading to the plasma effectively being trapped close to target surface causing low ion bombardment on the growing film which is a disadvantage for dense and good adhesion coatings. Instead of condensing the plasma near the target surface, the unbalanced
FIGURE 5.3: Drawing of a magnet configuration in DC magnetron sputtering showing race-track region (a) balanced magnetron and (b) unbalanced magnetron. (a)

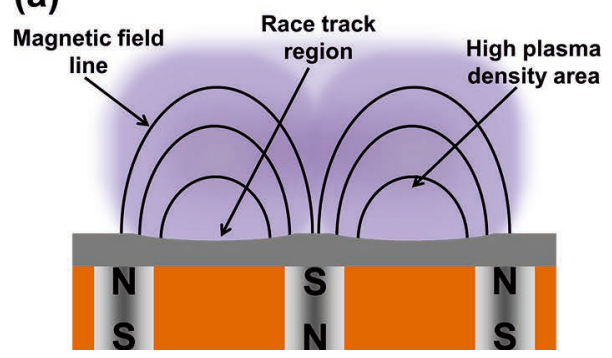

(b)

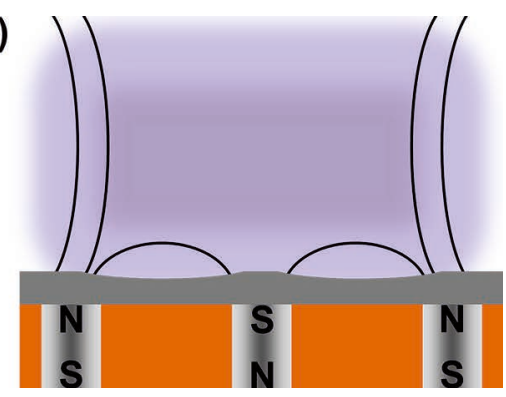




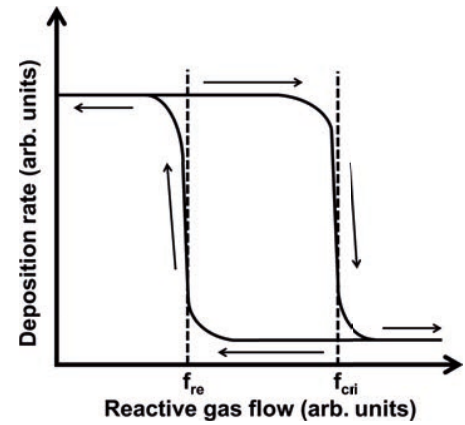

FIGURE 5.4: The deposition rate as a function of reactive gas flow. It is shown hysteresis behavior in reactive sputtering where $f_{\text {re }}$ is a recovery reactive gas flow and $f_{\text {cri }}$ is a critical reactive gas flow. Adapted from M. Ohring. 277 magnetron, on the other hand, allow electrons to escape from the confinement of electric and magnetic field cause the plasma in the region that further way from the target surface which leads to a better sputtered species transportation and ion bombardment yielding improvement of film quality. For type I, the inner magnets have higher strength than outer magnets. In type II configuration the outer magnets is stronger than the inner ones, i.e., the opposite to type I. Note that if the target is made from magnetic materials, the magnetic field from the target can interfere with the magnetron yielding inefficient electrons trapping. Thus, the advantage of using magnetron is gone. To solve this problem, we can engineer the shape of the target. Commonly thinner target is desired to allow magnetic field from the permanent magnet pass through the target or replace the permanent magnet and utilize the magnetization of target to confine electrons instead.

\subsection{Reactive sputtering}

Sputtering of compounds has issues with the stoichiometry of films, as result of a loss of certain sputtered species during transportation from target to substrate. In order to obtain good stoichiometric growth, reactive sputtering is often preferred. ${ }^{277,284}$ This technique uses sputtering from metallic target in reactive gas ambient. The target vapor phase species will react with reactive gas atoms which are cracked from its molecules by the plasma. This reaction takes place when sputtered species and reactive gas atoms arrive at the substrate surface and form compound films. No reactions occur during gas phase since that would require three-body collisions which is extremely improbable to occur because sputtering process has low pressure (about mTorr). Reactive sputtering is widely used for growth of oxides, nitrides, or sulfides for example. The reactive gas can react to the surface of the metallic target yielding a thin compound sheet on top of the target which is the so-called target poisoning effect. This sheet will affect the sputtering rate. In Figure 5.4 shows the so-called hysteresis curve of deposition rate against reactive gas flow rate that occurs during reactive sputtering of metallic targets. There are three different deposition modes, metallic mode, compound (poisoned) mode and transition mode which affect the deposition rate. In metallic mode, the sputtering system has a lot of metallic species than reactive gas, so film is deposited at very high rate 
(see Figure 5.4). This situation will be maintained until the system reaches an unstable reactive gas flow point which is called the critical flow point $\left(f_{\text {cri }}\right)$. If we still increase flow above this point, the deposition rate will suddenly drop in a non-linear or avalanche phenomenon. The extra reactive gas will form insulating sheet on top of target reduced bias voltage of target (target poisoning). As a result low $\mathrm{Ar}^{+}$ions bombard the target. This situation cannot reverse back to metallic mode easily by reducing reactive gas flow. The system has residual reactive gas which sticks at all chamber surfaces, target, and substrate. Thus time is needed for metallic species to consume reactive gas to return to metallic mode at the recovery reactive gas flow $\left(f_{\text {re }}\right)$. In order to prevent or reduce this situation, we can adjust the flow of reactive gas at the metallic mode in order to get high deposition rate. In case of using oxygen as reactive gas, one need to charge the supply cathode voltage from DC to radio frequency ac power or pulse DC power due to RF sputtering is compatible with insulator since each ac loop the ions in plasma will bombard and discharge to the target, so when the target is poisoning the sputtering process will continue with a little change. However, running reactive deposition under this condition makes it hard to control chemical composition of the film. Therefore, the method of partial pressure feedback control of the reactive gas was introduced. This method will allow us to operate reactive sputtering at the transition mode which is an intermediate mode between metallic and compound mode, therefore we can obtain the film that has correct stoichiometry with high deposition rate. 



\title{
Chapter 6
}

\section{Characterization techniques}

\begin{abstract}
"In physical science a first essential step in the direction of learning any subject is to find principles of numerical reckoning and practicable methods for measuring some quality connected with it. I often say that when you can measure what you are speaking about, and express it in numbers, you know something about it; but when you cannot measure it, when you cannot express it in numbers, your knowledge is of a meagre and unsatisfactory kind; it may be the beginning of knowledge, but you have scarcely in your thoughts advanced to the stage of science, whatever the matter may be."
\end{abstract}

Baron William Thomson Kelvin in From lecture to the Institution of Civil Engineers, London (3 May 1883)

This chapter will discuss and describe the characterization techniques that I have used in this Thesis.

\subsection{Structure characterization}

\subsubsection{X-ray Diffraction (XRD)}

$\mathrm{XRD}^{285,286}$ is one of the most useful techniques to characterize the structure of materials. XRD is used to identify the crystalline phases in a sample; structural properties can also be accurately determined: phase of the material, crystalline quality, strain state, grain size, epitaxial relationship, preferred orientation, and more. This technique is based on Bragg's law ${ }^{36,37}$ :

$$
n \lambda=2 d_{h k l} \sin \theta
$$

where $n$ is an integer corresponding to order of diffraction, $\lambda$ is the wavelength of the X-ray source, $d_{h k l}$ is the distance between 
lattice planes at given Miller indices $(h k l), \theta$ is the angle between the incident beam and the atomic plane. By diffraction of X-rays, there is a set of possible peaks depending on the crystal structure. In the present work, various XRD techniques using $\mathrm{CuK}_{\alpha}$ as X-ray source were used to do structural characterization of as deposited films.

\subsubsection{1 $\theta-2 \theta$ scan}

This is the most common mode in XRD. In the $\theta-2 \theta$ scan, the Xray tube movement is coupled with the detector movement, as we scan the X-ray beam with incident angle of $\omega=\theta$ and move the detector to detect diffraction X-ray at diffraction angle of $2 \theta$ (see Figure 6.1(a)). The scattering wavevector or momentum transferred to the crystal, $\mathbf{q}$, with $\mathbf{q}=\mathbf{k}_{\text {diff }}-\mathbf{k}_{\text {inc }}$, where $\mathbf{k}_{\text {inc }}$ is the incident X-ray wavevector which $\left|\mathbf{k}_{\text {inc }}\right|=2 \pi / \lambda$ and $\mathbf{k}_{\text {diff }}$ is the diffracted X-ray wavevector for the elastic scattering condition $\left|\mathbf{k}_{\text {inc }}\right|=\left|\mathbf{k}_{\text {diff }}\right|$, is changed during scanning with direction perpendicular sample surface. Once the magnitude of $\mathbf{q}$ fulfills the Laue condition ${ }^{36,37}$ (reaches the reciprocal lattice point in reciprocal space) that is

$$
\mathbf{q}=\mathbf{G}_{h k l},
$$

where $\mathbf{G}_{h k l}$ is the reciprocal lattice vector which equal to $\frac{2 \pi}{d_{h k l}} \hat{\mathbf{n}}$ and $\hat{\mathbf{n}}$ is the unit vector that is perpendicular to the $(h k l)$ lattice plane. Constructive interference will occur, thus the detector will detect diffracted X-ray so that there will be a peak appear on the screen (see Figure 6.1(b)). From the Equation (6.2), we can recover Bragg's law

$$
\begin{aligned}
\mathbf{q}=\mathbf{k}_{\text {diff }}-\mathbf{k}_{\text {inc }} & =\mathbf{G}_{h k l} \\
\left(\mathbf{k}_{\text {inc }}+\mathbf{G}_{h k l}\right)^{2} & =\mathbf{k}_{\text {diff }}^{2} \\
\left|k_{\text {inc }}\right|^{2}+2 \mathbf{k}_{\text {inc }} \cdot \mathbf{G}_{h k l}+\left|G_{h k l}\right|^{2} & =\left|k_{\text {diff }}\right|^{2} .
\end{aligned}
$$

Since $\left|\mathbf{k}_{\text {inc }}\right|=\left|\mathbf{k}_{\text {diff }}\right|$ and $\mathbf{G}_{h k l}=-\mathbf{G}_{h k l}$ (translational invariant in crystal lattice), thus

$$
2 \mathbf{k}_{\mathrm{inc}} \cdot \mathbf{G}_{h k l}=\left|G_{h k l}\right|^{2} .
$$

From Figure 6.1(a), we get $2 \mathbf{k}_{\text {inc }} \cdot \mathbf{G}_{h k l}=2\left|k_{\text {inc }}\right| \sin \theta$ and we know that $\left|\mathbf{G}_{h k l}\right|=2 \pi / d_{h k l}$, thus obtain the Bragg's law

$$
\begin{aligned}
2 \frac{2 \pi}{\lambda} \sin \theta & =\frac{2 \pi}{d_{h k l}} \\
2 d_{h k l} \sin \theta & =\lambda .
\end{aligned}
$$


Here, the detected peaks in the diffraction pattern are used to identify the crystalline phases present in the sample. Also we can determine the average grain size in the samples from the full width at half maximum (FWHM) of the diffraction peak intensity in $\theta-2 \theta$ scan due to the coherent scattering. The average grain size can be determine via Scherrer equation which is defined as

$$
D=\frac{K \lambda}{\beta \cos \theta},
$$

where $D$ is the average size of the ordered domain, $K$ is the dimensionless shape factor (typically $\sim 0.9$ ), $\beta$ is the full width at half maximum of diffraction peak intensity, $\lambda$ is the X-ray wavelength, $\theta$ is the Bragg angle.
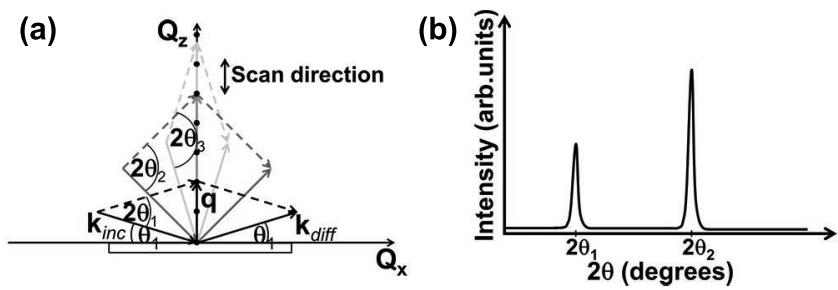

\subsubsection{2 $\omega$ scan or rocking curve measurement}

This scanning mode operates with X-ray tube and the detector fixed at $2 \theta_{h k l}$ where the Bragg condition is fulfilled, meaning that we are at the top of the diffraction peak of a certain lattice plane $(h k l)$. Then we scan $\omega$ which is not equal to $\theta$ like in $\theta-2 \theta$ scans (see Figure 6.2(a)), or we can keep the X-ray tube and the detector fixed at $2 \theta_{h k l}$ and "rock the sample" around the diffraction peak with angle of $\omega$. This type of scan observes the broadening (FWHM) of diffraction peak or reciprocal lattice point which will give us the information about crystalline quality. Since the broadening in $\omega$ scan occurs due to mainly mosaicity, dislocation, or curvature of the sample.

In the case of a perfectly aligned lattice plane crystal will produce a very sharp peak since there is no variation in $d_{h k l}$ (well define reciprocal lattice point), a little tilt in $\omega$ yields no diffraction peak since the Bragg condition is not met (see Figure 6.2(b)). The example of this is $\omega$ scan of a single crystal. Note that the perfect crystal still has broadening of diffraction peak due to instrument
FIGURE 6.1: X-ray scattering and momentum transfer in different condition (a) geometry symmetric, and (b) example result from $\theta-2 \theta$ scan. 
FIGURE 6.2: X-ray scattering and momentum transfer in (a) rocking curve or $\omega$ scan, and (b) example result from $\omega$ scan.
FIGURE 6.3: Illustration of Xray diffraction in pole figure measurement.
(I) In the non-perfect crystal, there are two types of intrinsic broadening, i.e., instrument and the domain size broadening. broadening ${ }^{\mathrm{I}}$. However, we can eliminate instrument broadening and the intrinsic width of the crystal grain by doing an $\omega$ scan on a set lattice plane since for example the broadening from mosaicity or dislocation will increasingly propagate in high order lattice plane and subtract the FWHM of high order with low order lattice plane, we will get only the contribution from mosaicity or dislocation.
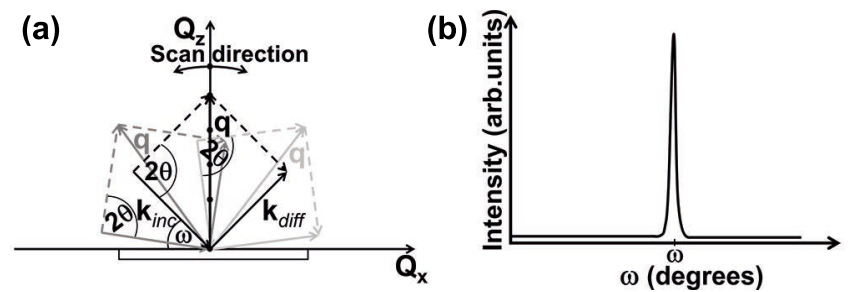

\subsubsection{Pole figure measurement}

To determine film texture, preferred orientation, epitaxial relation, or crystal symmatry identification, pole figures are used. The $\theta-2 \theta$ scan only gives reflection from lattice planes perpendicular to the plane normal. Sometimes, this result is not enough to identify the phase.

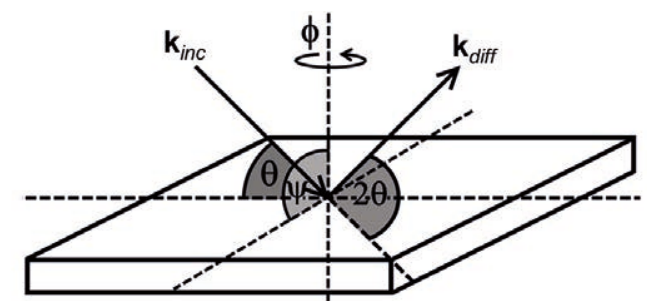

This is for example when the film is highly textured, i.e., the out-of-plane orientation of the crystal grain in the film is preferred, while in-plane orientation can be random (fiber texture) or aligned to substrate orientation (biaxial texture). To do a pole figure measurement, the $2 \theta$ angle of a set of lattice planes is fixed and the azimuth angle, $\phi$ in a range $0 \leq \phi \leq 360$ and tilt angle, $\psi(0 \leq \psi \leq 90)$. A set of sample orientations which fulfill Bragg's law ( $2 \theta$ fixed) can be mapped out from the intensity, $I(\psi, \phi)$. Since the sample is rotated with $\phi$, the information of 
crystal symmetry can also be obtained. The symmetry of the crystal is useful information to identify the materials. In the case of an epitaxial film on a substrate, the epitaxial relation can be obtained by the comparing in-plane direction between the film and the substrate using orientation measured from pole figure. If the tilt angle of certain set of lattice planes in the sample is known, one can perform only $\phi$ scan to find out the relation.

\subsubsection{Scanning Electron Microscope (SEM)}

$\mathrm{SEM}^{285}$ is a technique that acquires three-dimensional topographical surface images of a sample. Electrons can behave as waves the wavelength of which depends on it energy. SEM take advantage of this fact using electrons instead of light, which results in higher resolution and depth of field compared to Optical Microscope (OM). SEM is analogous with the reflective mode of OM. In SEM operation, high vacuum is required to prevent electron collision with the particles (gas molecules) before interact with the sample. Therefore the sample needs to be vacuum compatible. Electrons are emitted from electron gun, then focused electron beam by one or two electromagnetic condenser lens. After that the electron beam is scanned over the surface of sample. A detector collects the result of the interaction between the electron beam and the sample, e.g., secondary electrons (SE), X-rays, backscattered electrons (BSE), etc. The most common modes are imaging is secondary electrons (SE) mode, in which the surface topography is obtained by secondary electron contrast and backscattered electrons (BSE) that are used to both compositional contrast and topographical contrast. SEM is a simple method to understand topographical surface of the sample and does not require much time in sample preparation process. It is also easier to handle than Transmission Electron Microscope (TEM) since it does not require sample preparation before measurement if your material is electrical conducting. However, SEM has lower resolution compared to TEM due to low electron energy and the set up limitation.

\subsubsection{Transmission Electron Microscope (TEM)}

$\mathrm{TEM}^{287}$ is widely used for investigation of microstructure and composition of materials down to atomic scale (below $1 \AA$ ). TEM 
uses a high energy electron beam; typically the range of acceleration voltage in TEM is 60 to $300 \mathrm{kV}$ (compare to 5 to $20 \mathrm{kV}$ in SEM). Thus TEM provides very high resolution and large depth of field. TEM works in transmission mode like optical microscopes. The electron beam is accelerated from electron gun, then is focused by electromagnetic condenser lenses and aperture to control to intensity, intensity spread, convergent angle, current to get a coherence electron beam. The electron beam will penetrate a very thin sample $(<50 \mathrm{~nm})$. The scattering between the electron beam and the sample will occur. The transmitted and diffracted electron beam will focus by objective lens and gives crystallographic information due to electron diffraction patterns in reciprocal space on the back focal plane and the sample image in real space on the image plane. After being focused, these electron beams will go through the projection lenses system that consist of intermediate lens and projection lens to project the diffraction pattern or image onto a fluorescent screen or CCD camera. The image contrast in TEM can be produced by density or thickness of the sample (density and mass contrast), the diffraction of electrons (diffraction contrast), and the phase difference of electrons (phase contrast). The density and mass contrast occurs because electron beam is absorbed or backscattered by the samples (electron has small mean-free path). A dense or thick sample can absorb or backscatter more electrons than the soft or thin sample resulting in a different intensity of the image (contrast). The diffraction contrast occurs when coherent electron beam penetrates a very thin sample, some electrons directly transmit though the sample and some electrons are elastically diffracted due to atomic plane according to the Bragg's law. These electrons diffracted by the same set of atomic planes will then form a diffraction spot at back focal plane of objective lens. So if we take into account all the atomic planes, a diffraction pattern will form. Since these spots contain the image of sample, by selecting transmitted or diffraction spots (electron beam) in diffraction plane using objective aperture will result in a different contrast in the image. For transmitted spot (direct beam), it will give the lowest contrast image or no contrast (if there is no absorption or backscatter) since, for the ideal case all transmitted beams have the same intensity unless there is dislocation which scatters transmitted electron beam this will produce different intensity on the image; this image is called bright-field image. On the other hand, if we select the diffracted spots, since electrons 
differently diffract in the sample giving different intensity on the image; this image is called dark-field image. However, producing a dark-field image by selecting diffracted spots can produce a comatic image since the diffracted electron beam is not coherent. To correct this the center-dark field is introduced, we tilt the electron beam of a diffraction angle instead. Then the diffracted beam becomes transmitted beam which is more coherent. The last contrast is phase contrast. This contrast is used for producing high-resolution TEM image which is atomic volume projection of the crystal lattice. The term 'phase' comes from the phase of electron that is shifted when it interact with atomic potentials. After interactions, electrons will be transmitted or diffracted then they will inference at image plane producing a lattice fringes since electrons have different phase shift.

The spacial resolution in TEM can in principle go down to $1.9 \mathrm{pm}$ for $300 \mathrm{kV}$ acceleration voltage of electron. In practice, the resolution limited around $1 \AA$, because the resolution in TEM mostly is limited by electromagnetic lens aberration, i.e., chromatic $\left(C_{c}\right)$ and positive spherical $\left(C_{s}\right)$ aberration, i.e., electron beam has different focus plane causing the defocus when it is not coherent and has different convergent or scattering angle, respectively. The chromatic aberration can be reduced by having well-define electron gun and better condenser lenses system to have a coherent electron beam. For positive spherical aberration, nowadays there is a commercialized negative spherical corrector to compensate the positive spherical aberration. Despite to these corrections, there are a number of high order lens aberrations that limit TEM spacial resolution.

As mention earlier, the sample must be very thin for the electron beam to go through the sample. TEM sample preparation is time consuming. I prepare my samples by cutting with a low speed diamond saw into 2 small pieces of $1.8 \mathrm{~mm} \times 0.8 \mathrm{~mm}$ then put them on titanium grid and glue with araldite glue at $180{ }^{\circ} \mathrm{C}$ for 2 hours. Then the sample is polished with the diamond paper down to 50 $\mu \mathrm{m}$. After that the sample is ion milled to electron transparency with argon at $5 \mathrm{kV}$ and finish with low energy $(\sim 2 \mathrm{kV})$ ion beam in order to remove amorphous layer. 


\subsection{Compositional characterization}

\subsubsection{Energy-Dispersive X-ray Spectroscopy (EDX or EDS)}

The interaction between high energy electron beam and material produces characteristic X-rays from elements, like "fingerprint" of element. In EDX, ${ }^{285}$ the energy of characteristic X-ray is detected. If an electron microscope (SEM or TEM) is equipped with the Xray energy detector, the Energy Dispersive X-ray Spectroscopy (EDX/EDS) can carry out. Since characteristic X-rays is a fingerprint of elements, so we can use detected characteristic X-rays to qualitatively map out the composition of samples, and sometime the semi-quantitative composition in the samples can be mapped out in term of metals ratio. These qualitative analysis and semiquantitative element analyses are easily performed by EDX. By qualitative determination, the EDX is the best choice because it is fast. Moreover, we can use EDX not only for determining composition of the sample but also imaging. But EDX still has drawbacks in low resolution and accuracy. In addition, EDX is not suitable for light elements like $\mathrm{C}$ as well as $\mathrm{N}$, O or $\mathrm{H}$ which difficult to quantify, and also cannot obtain the type of bond or chemical shifts like X-ray Photoelectron Spectroscopy (XPS). Since, the type and amount of impurities can dramatically affect thermoelectric properties. Thus, I usually do further composition analysis with elastic recoil detection analysis (ERDA) which will be discussed in next section.

\subsubsection{Elastic Recoil Detection Analysis (ERDA)}

ERDA is a type of ion-beam analysis technique, which is used for compositional analysis in thin film. It is powerful and precise to analyze light element content or impurity in samples (such as $\mathrm{C}$, $\mathrm{N}, \mathrm{O}$, especially $\mathrm{H}$ ). Moreover, ERDA also give composition information in depth profiles of the samples. This is the advantage of ERDA measurements. The principle of ERDA is based on momentum and energy transfer, the sample is bombarded with hight energy heavy ion-beam hit and forward recoil atom of material inside sample. Commonly, iodine or chlorine ions with high energy about $40 \mathrm{MeV}$ are used. The incident angle of ion-beam is 
set typically $15-25^{\circ}$ from sample surface. The forward recoiled atoms and scattering incoming ions are detected at exit recoil angle, which relates to the incident angle (energy and momentum conservation). The Time-of-Flight Energy (ToF-E) detector is applied to distinguish forwarded recoil atoms by using the velocity and energy loss of the recoiled atom to quantify which element of atom is it. ERDA has some disadvantages, i.e., ERDA has low sensitivity to heavy atom since it is difficult to recoil, a tandem accelerator is necessary; also the evaluation is not trivial.

\subsection{Electrical characterization}

Therrmoelectric properties are very much dependent on the carrier concentration and mobility, especially their behavior as a function of temperature, which one could use to determine the transport mechanisms.

\subsubsection{Resistivity measurement}

Resistivity can be obtained by the four-point-probe technique which can be used on either bulk or thin film samples. ${ }^{288}$ The advantage of this setup is that the measurement is independent of contact and probe resistance, as long as size of probe is small compared to sample area. Figure 6.4 shows the four probes lined up and pressed against the sample surface; one pair of them measures current and another pair measures voltage.

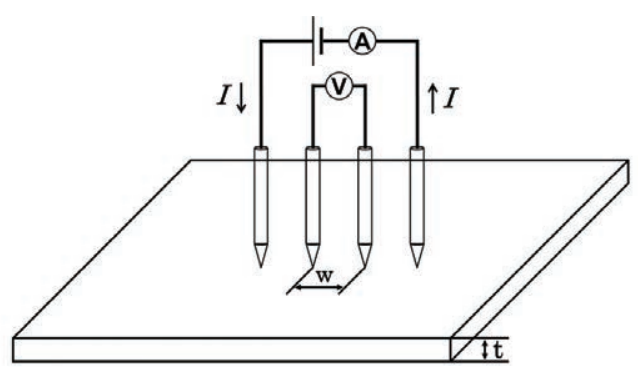

The sheet resistance, $R_{s}$ in a thin sheet can be calculated from

$$
R_{s}=\frac{\pi}{\ln 2} \frac{V}{I}=4.532 \frac{V}{I}
$$

FIGURE 6.4: Schematic drawing of four-point probe set up. 
FIGURE 6.5: Schematic drawing of the Hall effect in n-type semiconductor slab, Here $w$ is the width of slab, $d$ is the thickness of the slab, $B$ is magnetic field, I is current, $V_{H}$ is Hall voltage, and e is electrons. where $R_{s}$ is the sheet resistance, $I$ is an applied current, and $V$ is the measured voltage. This equation is valid for $t \leq s / 2$, where $t$ is the film thickness and $s$ is the space between probes.However, most samples are not effectively semi-infinite in either lateral or vertical dimension as we assume. Equation (6.7) needs to be modified to account for the finite geometry of samples. Thus we have to introduce geometry correction parameter, $F$ to correct the sheet resistance. For a rectangular sample, $F=F\left(\frac{b}{s}, \frac{a}{b}\right)$ where $a$ and $b$ is the length and width of sample, $s$ is the probe spacing. After measurement, sheet resistance must be multiplied by the film thickness to obtain the specific resistivity of film $(\rho)$. Thus, the specific resistivity is obtained from $\rho=R_{s} \cdot t$, where $\rho$ is the specific resistivity $(\Omega m$ or $\mu \Omega \mathrm{cm})$.

\subsubsection{Bulk carrier concentration and Hall mobility measurement}

When an electrical current is applied perpendicularly to a magnetic field on a piece of metal or semiconductor, the charge carriers in side those pieces experience force acting on them, changing their trajectories. The force is called as Lorentz force $\left(\mathbf{F}_{\mathbf{B}}\right)$ which is described as

$$
\mathbf{F}_{\mathbf{B}}=q(\mathbf{v} \times \mathbf{B}),
$$

where $q$ is electron charge ( $-e$ for electrons and $+e$ for holes), $\mathbf{v}$ is velocity of charge carriers, and $B$ is a magnetic field. The Lorentz force is a basic principle behind the Hall effect. ${ }^{36,37}$ An electrical current $(I)$ is applied on a bar-shaped n-type semiconductor along the $\mathrm{x}$-axis from left to right in the presence of a perpendicular magnetic field in $z$ direction (Figure 6.5). The free electrons inside the semiconductor interact with magnetic field and drift away from their direction toward the upper side of the slab due to the Lorentz force.

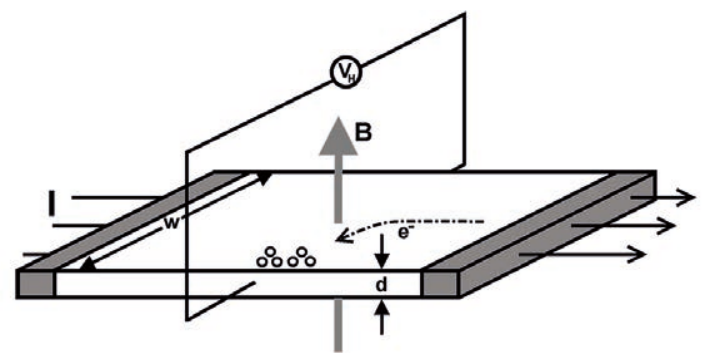


Note that the force that acts on holes will push holes to the same direction as electrons because they have opposite velocity direction and positive charge. This results in a charge accumulation on the side of the slab, yielding the voltage drop across on two sides which is called the Hall voltage, $V_{H}$ (negative for n-type semiconductor and positive for p-type semiconductor). ${ }^{31}$ The Hall voltage is determined by

$$
V_{H}=\frac{I B}{q n d}
$$

where $I$ is the applied current, $B$ is the magnitude of applied magnetic field, $d$ is the sample thickness, and $q$ is the electron charge. Thus, we can determine the bulk charge carrier concentration, $n$ from

$$
n=\frac{I B}{q d V_{H}}
$$

If the sheet resistance $R_{S}$ is known, the carrier Hall mobility of the sample can be obtained from

$$
\mu=\frac{\left|V_{H}\right|}{R_{s} I B}=\frac{1}{q n_{s} R_{s}}
$$

where $n_{s}$ is sheet carrier concentration and the electrical resistivity ( $\rho$ ) can be determined by $\rho=R_{s} d$. To determine both the mobility and the bulk charge carrier concentration at the same measurement, a combination of electrical resistivity and Hall voltage measurement is required.

Thus, the van der Pauw technique is used, ${ }^{288}$ a convenient method widely used to measure the resistivity of uniform samples because this technique requires less sample preparation only four tiny ohmic contacts placed in the corners of the thin samples. In order to obtain the sheet resistance $\left(R_{S}\right)$, van der Pauw showed that the sheet resistance is related to two characteristic resistances $R_{A}$ and $R_{B}$ through the van der Pauw equation. ${ }^{288}$

$$
e^{\left(-\pi R_{A} / R_{S}\right)}+e^{\left(-\pi R_{B} / R_{S}\right)}=1
$$

This means if one could measure two resistances corresponding to terminals shown in Figure 6.6, the sheet resistance can be solved numerically and the electrical resistivity can be determined by $\rho=R_{s} d$. From the figure, we can determine $R_{A}$ and $R_{B}$ by applying a current $(I)$ to a pair of contacts and measure voltage from another pair, thus the $R_{A}$ and $R_{B}$ are calculated from

$$
R_{A}=V_{43} / I_{12} \quad \text { and } \quad R_{B}=V_{14} / I_{23}
$$
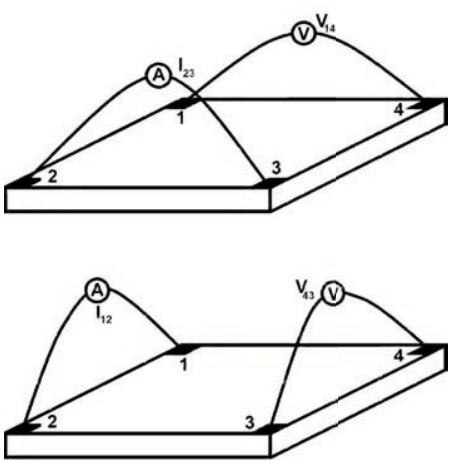

FIGURE 6.6: Schematic of van der Pauw configuration for resistivity measurement in square sample.

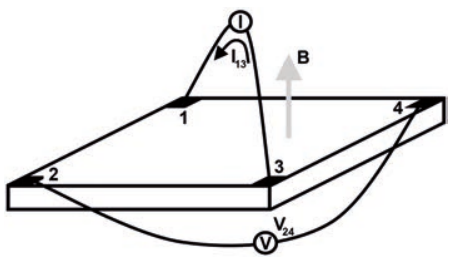

FIGURE 6.7: Schematic of van der Pauw configuration for Hall measurement in square sample. 
(a)

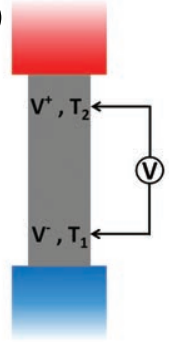

(b)

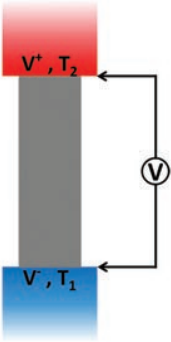

According to the configuration of the contacts, we can use it to obtain Hall voltage $\left(V_{H}\right)$. In order to measure the Hall voltage, a constant current $(I)$ is applied between a pair of contacts placed opposite from each other in a presence of perpendicular magnetic field $(B)$ and the Hall voltage is measured across the other pair (see Figure 6.7). Therefore, we can use Equation (6.10) to obtain the charge carrier concentration $(n)$ and Equation (6.11) for the carrier Hall mobility $(\mu)$.

FIGURE 6.8: Schematic drawing of probes configurations for Seebeck measurement, (a) the potentiometric (four probes) and (b) the axial-flow (two probes) configuration, where $\mathrm{T}_{1}<\mathrm{T}_{2}$ (adapted from ref.[27]).

\subsubsection{Seebeck coefficient measurement}

Assume two materials are physically and chemically homogenous and isotropic with a good Ohmic and isothermal contact, the $V_{\mathrm{AB}}$ will depend on hot and cold temperature explicitly and independent of the temperature distribution between interface. This is called the empirical Law of Magnus. ${ }^{26,27}$ For $T_{2}<T_{1}$, we can show that Seebeck coefficient is linearly dependent with $T$,

$$
V_{\mathrm{AB}}=\left(S_{\mathrm{B}}-S_{\mathrm{A}}\right)\left(T_{2}-T_{1}\right)=S_{\mathrm{AB}} \Delta T .
$$

The basic set up for Seebeck coefficient is shown Figure 6.8. In the axial-flow technique, the temperature and voltage difference are measured on the probes which are placed inside the heat source and sink contact to the end of the sample. ${ }^{27}$ In the potentiometric technique, the temperature and voltage difference are measured at two points on the middle of sample and on the axis parallel to temperature gradient, other two probes are placed inside the heat source and sink. Note that the diameter of temperature/voltage probes needs to be smaller than the distance between them to obtain accurate results. Generally, there are two main methods, i.e., integral and differential method, used to obtain Seebeck coefficient. The integral method (large $\Delta T$ ), the temperature at one end of the sample is fixed, $T_{1}$ while the temperature at other end is varied through the temperature of interest via $T_{2}=T_{1}+\Delta T$ then the voltage that is generated by that temperature gradient is measure accordingly. To obtain the Seebeck coefficient, fitting is applied to the entire data set $V_{\mathrm{ab}}\left(T_{1}, T_{2}\right)$ then differentiated with respect to $T_{2}$, so the Equation (6.14) becomes

$$
S_{\mathrm{AB}}\left(T_{2}\right)=S_{\mathrm{B}}\left(T_{2}\right)-S_{\mathrm{A}}\left(T_{2}\right)=\frac{d V_{\mathrm{AB}}\left(T_{1}, T_{2}\right)}{d T_{2}} .
$$

The purpose of this thesis is finding good mid-high temperature thermoelectric materials, therefore we do not use the integral 
method because it is not appropriate on high temperature measurement because of the difficulty to maintain isothermal contacts through. On the other hand, the differential method (small $\Delta T$ ) is a proper method to determine high temperature Seebeck coefficient. In the differential method, a small thermal gradient $\Delta T$ is applied across a sample. The temperature of interest is maintained at mean temperature $T_{m}=\frac{\left(T_{1}+T_{2}\right)}{2}$, where $T_{1}=T_{m}-\left(\frac{\Delta T}{2}\right)$ and $T_{2}=T_{m}+\left(\frac{\Delta T}{2}\right)$. To avoid nonlinearity problems and eliminate the offset voltage from inhomogeneites and nonequilibrium of contact interface, one has to choose temperature interval $\Delta T$ small enough giving $V_{\mathrm{AB}} \propto T_{0}$. Thus, the Seebeck coefficient is linearly dependent with $T$, so that it can be simply determined by slope of voltage versus temperature difference, where Equation (6.14) becomes

$$
\frac{\Delta V_{\mathrm{AB}}}{\Delta T} \simeq S_{\mathrm{AB}}\left(T_{m}\right) .
$$

There are three differential methods often use to calculate the Seebeck coefficient from the linear fit of the data points which are depended on the time interval for performing one voltage measurement. Those thee methods are steady-state $(d c)$, quasisteady-state $(q d c)$, and transient ( $a c$ ) condition during measurement. For the steady-state condition, the stable temperature difference across specimen is required before measuring electrical voltage. Therefore, the steady-state is successful in reducing the offset voltages, however, one have to face the time consuming problem due to stabilization of temperature difference for each point of measurement. However, the absolute Seebeck coefficient of each material needs to be determined. It can be done by using a set of reference wires (including a set of thermocouple) whose absolute Seebeck coefficients were already obtained from Thomson effect. The most used materials for high temperature measurement are $\mathrm{Cu}$ and $\mathrm{Pt}$, for Pt the most accurate reference data has been obtained by Roberts with estimates uncertainty $\pm 0.2 \mu \mathrm{VK}^{-1}$ at the highest temperature. ${ }^{289}$

Seebeck coefficient measurement can be combined with the fourpoint probe technique where two probes are placed at both end of the sample and the another two probes will be placed at the center for voltage measurement. This configuration will allow us to obtain both Seebeck coefficient and resistivity of the sample simultaneously. Since a temperature gradient is applied to get 
Seebeck coefficient. In order to obtain the "correct" voltage for resistivity measurement, the fast switching current polarity generator is used to supply the current into sample, then we will average the measured voltages to avoid the arise of voltage due to Seebeck effect, excess voltage from Peltier effect, and inhomogeneous current flow that are measured during experiments. Thus the measured voltage drop across sample is

$$
V_{\mathrm{IR}}=\frac{\left(V_{I^{+}}+S \Delta T\right)-\left(V_{I^{-}}+S \Delta T\right)}{2}
$$

In this thesis, ZEM-3 is used to measure Seebeck coefficient. It uses the differential method with steady-state condition operated upon potentiometric technique to obtain both sheet resistivity and Seebeck coefficient. Two R-type thermocouples are used as a voltage and temperature gradient measurement probes with distance between probes of $4 \mathrm{~mm}$. The other two probes are Pt connect to nickel-base connected to both end of the film with sliver paste for passage of current through the film which lower probes also connect to heater for generating temperature gradient across to the film. To increase an average temperature nickel shield is cover all of described component with thermocouple on the side to determine temperature. The sample is inserted into a furnace pumped to vacuum condition with a small amount of He gas. The Seebeck is obtained by linear fit of three $\Delta T$ intervals, e.g., 10, 20 and 30 K.

\subsubsection{Time-Domain Thermoreflectance (TDTR)}

Measuring thermal conductivity of thin film samples which is the great challenge. Since a thin film (about hundreds of nanometer thick) samples have to grow on a substrate, the thermal conductivity of substrate will dominate the heat flow meaning that we cannot obtain the stable temperature gradient across the thin film sample. There are a few techniques that were developed to solve this issue such as the hot wire method, $3-\omega$ method and the timedomain thermoreflectance. ${ }^{290}$

TDTR is used to determine thermal conductivity of our thin film samples. ${ }^{290}$ This technique uses the idea of an acoustic strain wave that is generated when the materials relax from thermal stress by the thermal expansion due to a local heating from an optical pulse. This acoustic strain wave will later propagate in 
material then it will transmit and reflect at the interface between film and substrate. This acoustic strain wave that travel inside the material can change the refractive index in the material. This effect is so-called the piezo-optic effect. We can detect this effect from the change in surface reflectivity $\Delta R$ when that acoustic strain wave reflect back to the surface. Therefore, if we can synchronize the two laser beams that one is used to heat up the sample (pump beam) and later another one is used to probe the change in $\Delta R$ of the sample (probe beam). So, we can obtain the amount of temperature change from

$$
\Delta T(z)=(1-R) \frac{Q}{C(\xi A)} e^{-z / \xi},
$$

where $R$ is the sample reflectivity, Q is the optical beam energy, C is the specific heat per unit volume, A is the optical spot area, $\xi$ is the optical absorption length, and $\mathrm{z}$ is the distance into sample. TDTR uses the piezo-optic effect ${ }^{290}$ to obtain thermal conductivity of thin film samples by fitting the change of reflectivity of a thin metal layer, typically $\sim 100 \mathrm{~nm}$ of Al that was put on top of the thin film sample ${ }^{\mathrm{II}}$ Here the experiment is done by following: a short and low energy pump pulsed laser beam (typically $\sim 100$ $n s$ and $\sim 1 n \mathrm{~J}$ ) is focused on the metal film to heat up for a few Kelvin. After absorption of a pump laser pulse for a few nanoseconds, the metal film will cool down due to heat propagation passing through interface into film sample. By using the probe pulsed laser beam (usually has the same pulsed width as pump beam) to detect the $\Delta R$ thin metal, then we can obtain the rate of cooling via Equation (6.18) as shown in Figure 6.9. Then this measurement data will be fitted to the heat diffusion differential equation which is defined as

$$
\kappa_{s} \frac{\partial^{2} T_{s}(z, t)}{\partial z^{2}}=c_{s} \frac{\partial T_{s}(z, t)}{\partial t},
$$

where $\kappa_{s}$ is the thermal conductivity of thin film sample, $c_{s}$ is the specific heat per unit volume of thin film sample, $T_{s}(z, t)$ is the function of temperature of thin film sample at position $z$ and time $t, z$ is the distant into the film, and $t$ is the represent time. Here we assume that the lateral heat flow is smaller than the heat flow into the sample. To solve this partial differential equation we need two boundary conditions as

(i) the rate of the metal film loses temperature must be equal to the energy flux into the sample film :
(II) Here I discuss the technique in a simple version by using heat diffusion differential equation in the Cartesian coordinate to illustrate the concept of the technique. TDTR has been improved in a past decade in terms of using the heat diffusion equation in cylindrical coordinate instead of Cartesian coordinate and combined the fact that the TDTR signals are measured as a function of the modulation frequency and the laser repetition rate of laser pulses as the "pulse accumulation" model as the the data analysis corrections. Moreover we can modulate the frequency instead of time delay which is called Frequcy-domain thermoreflectance (FDTR). 291-293 


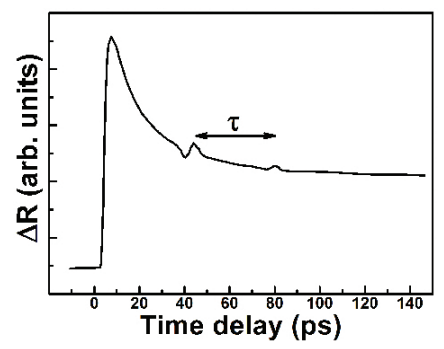

FIGURE 6.9: The time-domain thermoreflectance measurement data of aluminum thin film deposited on a silicon substrate. The plot shows the change in the reflectivity $(\Delta R$ ) with respect to the laser probe-pulse time delay. The time difference between two peak $(\tau)$ is the time for acoustic pulse travel twice thickness of the aluminum thin film. Thus the thickness of aluminum film can be determined from $\tau=2 d / v_{L}$, where $\mathrm{d}$ is the thickness of the thin film and $v_{L}$ is the longitudinal sound velocity in the thin film. Adapted from ref. [290].

$$
\left.\kappa_{s} \frac{\partial T_{s}(z, t)}{\partial z}\right|_{z=0}=c_{m} d \frac{\partial T_{m}(z, t)}{\partial t}
$$

and

(ii) the temperature drop at boundary between thin film sample and metal film is related though the heat flux crossing at boundary :

$$
\left.\kappa_{s} \frac{\partial T_{s}(z, t)}{\partial z}\right|_{z=0}=-\frac{T_{m}(0, t)-T_{s}(0, t)}{R_{K}},
$$

where $d$ is the thickness of metal film, $c_{m}$ is the specific heat per unit volume of metal film, $T_{m}(z, t)$ is the temperature of metal film at position $z$ and time $t$, and $R_{K}$ is the thermal-boundary resistance between thin film sample and metal film. In order to solve these equations, we must know following parameter accurately, the thickness of metal film $d$, the specific heat per unit volume of metal film and thin film sample $c_{m}$ and $c_{s}$. Then we vary thermal conductivity of thin film sample $\kappa_{s}$ and thermal-boundary resistance $R_{K}$ until the solution fit the measurement data.

However, if the thickness of the thin film sample is thinner when compared to thermal diffusion length (penetration depth) of thermal waves $\delta$ defined as $\delta=\sqrt{\kappa_{s} \tau_{\max }} / c_{s}=\sqrt{\kappa_{s} / \pi c_{s} f}$, where $\tau_{\max }$ is the maximum time delay of the probe beam with respect to the pump beam, $f$ is the frequency of the probe beam with respect to the pump beam, then the heat from the pump beam can flow into the substrate causing the error in our fitting results. Therefore, the correction to our model need to be make. This can be done by generating another set of heat diffusion differential equations for thin film sample and substrate with three additional accurately known parameter which are $l$ is the thin film sample thickness, the specific heat per unit volume of substrate $c_{\text {sub }}$, and thermal conductivity of the substrate $\kappa_{\text {sub }}$. Then guessing the thermalboundary resistance $R_{K}$ until the solution fits the measurement.

Here we can define a term called "thermally thin" when the thin film sample thickness smaller than $\delta$ and "thermally thick" when the thin film sample thickness larger than $\delta$. For the thermally thin sample, the uncertainty of measurement will come the modeling of thin film and substrate thermal conductance, since it is difficult to avoid. On the other hand, the thermally thick sample, the uncertainty comes from how well estimation of sample specific heat 
capacity since the error of this estimation will linearly propagates into $\kappa_{s}$. Typical uncertainties of $\kappa_{s}$ which is determined by TDTR are around $5-10 \%$.

For the measurement setup (see Figure 6.10), the Ti:sapphire laser oscillator that produces a train of femtosecond laser at high repetition about $80 \mathrm{MHz}$, is usually used in TDTR measurement. However there are alternative such as $1.55 \mu$ m wavelength Er:fiber, $1.05 \mu \mathrm{m} \mathrm{Yb:fiber,} \mathrm{Yb:tungstate,} \mathrm{Nd:glass} \mathrm{are} \mathrm{also} \mathrm{used.}$ The reason for using these fiber laser oscillators are the smaller footprint and lower cost than Ti:sapphire one. ${ }^{292}$ Then the output of the laser source will be split into two paths i.e., pump and probe path, by a polarizing beam splitter. This polarizing beam splitter is also used to control the power of the probe and pump beam by adjusting the polarization angle of the beam where the pump beam has higher power than the probe since the pump beam has to heat the metal film. The pump beam will continue to an electro-optic modulator (EOM) which is used to chop undesired frequencies of the pump beam (typically at frequencies beyond $10 \mathrm{MHz}$ ) to avoid the overheating effect on the thin film sample by the unmodulated pump beam. The pump beam has to pass through optical delay line which can consist of a retroreflector or mirror put on a mechanical stage in order to create the time delay between pump and probe beam, so that we perform both heating and measuring the dependence of optical reflectivity in metal film transducer at the temperature, $\frac{d R}{d T}$ with pump and probe beam, simultaneously. After this stage the pump beam is focused by optical lens on the metal film transducer.

For the probe beam, it will pass though a audio-frequency mechanical chopper to remove the coherent background signals. Then the probe beam will be focused on the sample spot as the pump beam. The pump and probe spot size typically around 10$50 \mu \mathrm{m}$ in diameter. The reflected probe beam will go to a high bandwidth photodetector. The output will send to a lock-in amplifier where the reference signal has the same frequency as EOM. The voltage output from lock-in amplifier will be proportional to $\Delta R$. Then measuring this signal with the changed of mechanical stage in order to get a relation between $\Delta R$ and the optical probe-pulse time delay. This shows that TDTR is a good technique to measure thermal conductivity of thin films. However, there are some limitations we need to consider. First the roughness of the 
FIGURE 6.10: The schematic drawing of the time-domain thermoreflectance setup. Adapted from ref. [290].

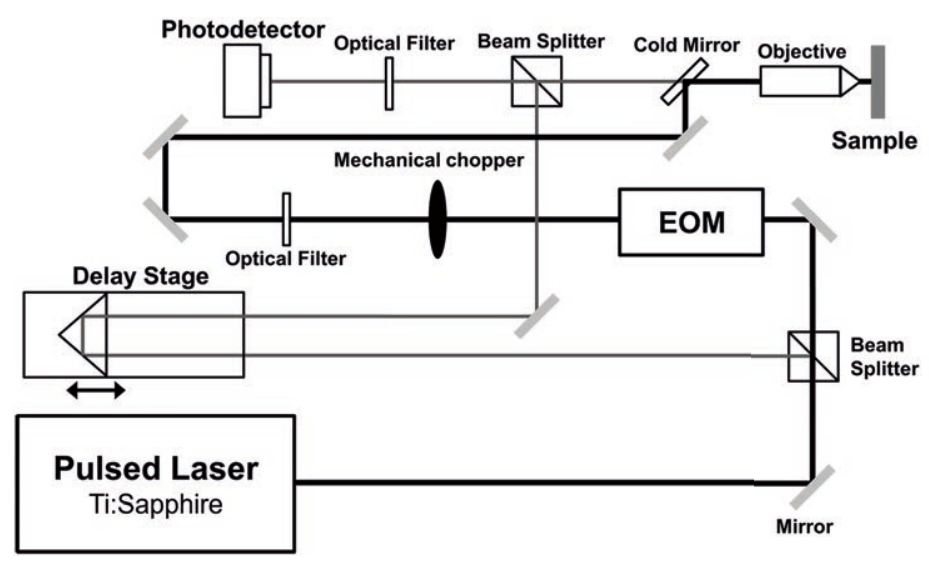

surface sample should be lower than $15 \mathrm{~nm}$, this is to avoid the diffuse scattering of acoustic strain wave at the interface between thin film sample and metal film transducer. ${ }^{292}$ Second, TDTR has difficulty in measuring in-plane thermal conductivity. This is due to the nature of this technique that TDTR has only sensitivity in the direction that perpendicular to the film surface (or cross-plane thermal conductivity). This is due to the failure of Fourier theory in TDTR measurements. ${ }^{49}$ Therefore, the careful data analysis is required to study the anisotropy thermal conductivity of materials by TDTR. ${ }^{292}$ The third limitation is TDTR cannot measure below $30 \mathrm{~K}$ because we cannot keep the temperature gradient of the metal film transducer. Because the metal has small heat capacity, we need to reduce the laser power to keep the temperature gradient stable. For the low temperature measurement, the lowest temperature that we can use TDTR is depended on the ambient absolute temperature, $T$ multiply the heat capacity per unit volume of metal film transducer, $C .{ }^{292}$ For the high temperature measurement, there is no such a limitation. But there is a possibility that the metal film transducer may physically or chemically interact with the thin film sample at high temperature which can cause dramatic errors on the results. ${ }^{292}$ So we should select an appropriate metal film transducer for specific measurement. A details discussion of current TDTR measurements can be found in ref. [292, 294]. 

痛みを伴わない教訓には意義がない。人は何 かの犠牲なしには何も得ることはできないの だから。しかしその痛みに耐之、乗り越之た とき人はなにものにも負けない強勒な心を手 に入れる。そう鋼のような心。

Itami wo tomonawanai kyokun niwa igi ga nai. Hitowa nanika no gisei nashi niwa nanimo eru kotowa dekinai no dakara. Shikashi sono itamini tae, norikoeta toki hitowa nanimononimo makenai kyoujin na kokoro wo teni ireru. Sou haganeno youna kokoro.

There's no such thing as a painless lesson-they just don't exist. Sacrifices are necessary. You can't gain anything without losing something first. Although if you can endure that pain and walk away from it, you'll find that you now have a heart strong enough to overcome any obstacle. Yeah... a heart made Fullmetal.

Said by Edward Elric in the last episode of Fullmetal Alchemist Brotherhood Hiromu Arakawa, Original Creator 


\section{Chapter 7}

\section{Summary and my contributions to the field}

This Thesis is focused on fundamental research about understanding thermoelectric properties of $\mathrm{ScN}, \mathrm{CrN}$ and their solid solid solutions, and possibility to improve their thermoelectric figure of merit $(Z T)$. To achieve these goals, I employed both experimental and theoretical methods to investigate their thermoelectric properties in connection with structural, and chemical composition.

$\mathrm{ScN}$ thin films in this Thesis were grown by reactive dc magnetron sputtering on $\mathrm{Al}_{2} \mathrm{O}_{3}(0001)$. I discovered that $\mathrm{ScN}$ has high power factor $2.5 \times 10^{-3} \mathrm{Wm}^{-1} \mathrm{~K}^{-2}$ at $800 \mathrm{~K}$, due to low metalliclike electrical resistivity $\left(\sim 3 \times 10^{-6} \Omega \mathrm{m}\right)$ with retained relatively large Seebeck coefficient $-86 \times 10^{-6} \mathrm{VK}^{-1}$. This power factor is unexpectedly large for a transition-metal nitride and similar in magnitude to state of the art thermoelectric materials such as $\mathrm{Bi}_{2} \mathrm{Te}_{3}$ or PbTe. ${ }^{84,295}$ Following studies show that the power factor of ScN is dependent on their composition. The power factor of $\mathrm{ScN}$ can be changed by one order of magnitude depending on e.g. oxygen, carbon, and fluorine content. [Paper I]

In order to understand why $\mathrm{ScN}$ has such a high power factor with high sensitivity to impurities and find a possibility to improve that power factor, I used density functional theory (DFT) calculation with supercell approach to study the effect of substoichiometry and impurities on electronic density of states (DOS) of ScN. The general gradient approximation (GGA) has been chosen to use as the main exchange correlation energy in this study. GGA requires limited computing resources which is good for this study because we need a large supercell to obtain doping concentration close to the experiment value. In addition, it is a reasonable approximation to describe $\mathrm{ScN}$, as it gives lattice parameter close to the experimental value, ${ }^{127,128,296}$ despite the fact that GGA give an incorrect band gap. The results show that $\mathrm{Sc}$ and $\mathrm{N}$ vacancies can introduce asymmetric peaks in the density of states close to the 
Fermi level with a shift of the Fermi level. In addition, substitutional point defects can shift the Fermi level in the electronic band according to their valence but do not introduce sharp features. The band gap problem with GGA, was addressed by using hybrid functionals. The results from hybrid functional calculations show that $\mathrm{ScN}$ has $0.9 \mathrm{eV}$ band gap unlike GGA calculation that show zero point band gap. Introducing $\mathrm{N}$ vacancy gives a sharp peak in the middle of energy gap with Fermi level is shifted. The vacancies induce the sharp peak in ScN DOS and impurities shift the Fermi level, which could be the reason why ScN has substantially high thermoelectric power factor following the Mahan-Sofo suggestion. ${ }^{77}$ This study also suggest the possibility pathway for improving power factor of thermoelectric materials by introducing an appropriate dopant with the presence of vacancies to move the Fermi level near the location of the vacancy peak in the electronic density of states. [Paper II]

However, ScN still has high thermal conductivity, which is undesirable for thermoelectric applications. To address this issue, I conducted a theoretical and experimental study on the effect of the microstructure on the lattice thermal conductivity of $\mathrm{ScN}$, using a theoretical model based on molecular dynamics (developed by Hellman et al. ${ }^{260,261,270}$ ). The results show a trend of lattice thermal conductivity versus grain size with good agreement between theoretical and experimental results. There is a systematic difference about $30 \%$ in the magnitudes since the model does not include the impurity scattering effect and the type of grain boundary interface. Yet, we demonstrate that it is a good model to predict the effect of the microstructure on the lattice thermal conductivity. Moreover, the results from this study also provide a possibility to control thermal conductivity by tailoring the microstructure of ScN thin film. [Paper III]

Another way to reduce thermal conductivity is forming a solid solution. Here I selected $\mathrm{CrN}$ to form solid solution with ScN. CrN is a transition-metal nitride like $\mathrm{ScN}$, but with higher hardness and corrosion resistance. In term of thermoelectric properties, at room temperature $\mathrm{CrN}$ has large Seebeck coefficient of $-135 \times 10^{-6}$ $\mathrm{VK}^{-1}$ and low thermal conductivity of $1.76 \mathrm{~W}(\mathrm{mK})^{-1}$, but it has high electrical resistivity of about $1.7 \times 10^{-5}$ to $3.5 \times 10^{-4} \Omega \mathrm{m}$. This large Seebeck coefficient comes from $\mathrm{Cr}$ has heavy effective mass since it has localized $3 d$ orbitals. This large effective mass re- 
sults in a high electrical resistivity. By forming solid solution with $\mathrm{ScN}$, we can exploit the empty $3 d$ orbitals in Sc to delocalize electrons in Cr $3 d$ orbitals, resulting in electrical resistivity reduction and possibly decreased thermal conductivity due to alloying element phonon scattering. Here I performed phase stability calculations of $\mathrm{Cr}_{1-\mathrm{x}} \mathrm{Sc}_{\mathrm{x}} \mathrm{N}$ disordered solid solutions and their electronic DOS by DFT with Localized Density Approximation with Hubbard $\mathrm{U}$ term $(\mathrm{LDA}+\mathrm{U})$. The choice of $\mathrm{LDA}+\mathrm{U}$ is because we consider the strongly correlate system that is CrN. Here we selected the value $3 \mathrm{eV}$ for the effective $U^{\text {eff }}$ applied on the $3 d$ orbitals of $\mathrm{Cr}$ and Sc. This value was found to be optimal for reproducing both the experimental lattice parameter of the paramagnetic phase and the experimental valence band electronic structure of the cubic phase ${ }^{174}$. Moreover I also did the calculated $\mathrm{ScN}$ with the same $U^{\text {eff }}$. The results show that the calculated lattice parameter closely match with the experimental value. The theoretical results show that $\mathrm{Cr}_{1-\mathrm{x}} \mathrm{Sc}_{\mathrm{x}} \mathrm{N}$ disordered solid solutions are stable and the correspond DOS suggest a possible improvement on thermoelectric properties due to the delocalization of electrons in $3 d$ orbitals. The experimental results show that we can synthesize $\mathrm{Cr}_{1-\mathrm{x}} \mathrm{Sc}_{\mathrm{x}} \mathrm{N}$ solid solutions thin films on $\mathrm{Al}_{2} \mathrm{O}_{3}(0001)$ by reactive magnetron sputtering, these results inline with the prediction from the theory calculations. The power factor of $8 \times 10^{-4} \mathrm{Wm}^{-1} \mathrm{~K}^{-2}$ at 775 $\mathrm{K}$ for $\mathrm{CrN}$ can be retained for the solid solution $\mathrm{Cr}_{0.92} \mathrm{Sc}_{0.08} \mathrm{~N}$. This shows a possible improvement of $Z T$ due to the possibility of lattice thermal conductivity reduction via phonon alloy (Sc) scattering. [Paper IV]

I also discuss present-day strategies on how to improve $Z T$ of materials. These strategies involve mainly forming solid solutions, nano-inclusion, or superlattices. Since a large temperature gradient has to be applied on thermoelectric material, this gradient can activate diffusion. The superlattice might intermix, solid solutions could order or phase separate, and nano-inclusion might be dissolved in the matrix. These processes could positively or negatively change thermoelectric properties of engineered materials. In order to choose the best strategies to engineer thermoelectric properties of ScN, I used again DFT to study phase stability of ScN and its solid solutions. 13 different nitrides and 3 other pnictides selected as candidates. From this study, I can suggest strategies to decrease thermal conductivity of ScN-based systems. 
For the solid solutions, $\mathrm{Sc}_{1-\mathrm{x}} \mathrm{Lu}_{\mathrm{x}} \mathrm{N}$ is interesting candidate, since it shows stability as disordered solid solution. Thus it has a possibility of decrease thermal conductivity due to phonon alloy scattering while retaining power factor because the mass difference between Sc and Lu is large. They have the same valence electrons meaning that Seebeck coefficient will be maintained when $\mathrm{Lu}$ is added into ScN since there is no doping effect. For superlattices, Indium is good candidate due to phase separation tendency with ScN. Moreover it also has a large mass difference compared to Sc. The reason for $\mathrm{Y}$ and La are not selected to form superlattice with $\mathrm{ScN}$ is that both YN and LaN are easily oxidized which can cause delaminate of the superlattice film due to high stress from the expansion of oxidized layer. In addition, I also found an interesting results for the Sc-Nb-N and Sc-Ta-N systems. The inherently layered ternary structures $\mathrm{ScNbN}_{2}$ and $\mathrm{ScTaN}_{2}$ are stable which open up a new field of study for thermoelectric applications. The Sc-V$\mathrm{N}$ system also exhibits the same tendency as Sc-Nb-N and Sc-Ta-N systems but not as pronounced. [Paper V]

According to all these findings and suggestions, on a personal level I can consider I achieved the aims that were set in the beginning of my Ph.D study. I have proposed a new class of materials, transition-metal nitrides for thermoelectric applications. I also suggest pathways to further improve thermoelectric properties of transition-metal nitrides. These pathways are not only applicable to transition-metal nitrides, but can be applied to any material in general. 




\section{Bibliography}

[1] I. E. A. (IEA), World Energy Outlook 2014. France: OECD/IEA, 2014.

[2] S. Bigerna, C. A. Bollino, and S. Micheli, The Sustainability of Renewable Energy in Europe. Switzerland: Spinger International Publishing, 2015.

[3] F. J. DiSalvo, "Thermoelectric Cooling and Power Generation," Science, vol. 285, p. 703, 1999.

[4] G. J. Snyder and E. S. Toberer, "Complex thermoelectric materials," Nat. Mater., vol. 7, pp. 105-114, 2008.

[5] C. J. Vineis, A. Shakouri, A. Majumdar, and M. G. Kanatzidis, "Nanostructured Thermoelectrics: Big Efficiency Gains from Small Features," Adv. Mater., vol. 22, pp. 3970-3980, 2010.

[6] A. Shakouri, "Recent Developments in Semiconductor Thermoelectric Physics and Materials," Annu. Rev. Mater. Res., vol. 41, pp. 399-431, 2011.

[7] T. M. Tritt, "Thermoelectric Phenomena, Materials, and Applications," Annu. Rev. Mater. Res., vol. 41, pp. 433-448, 2011.

[8] C. B. Vining, "An inconvenient truth about thermoelectrics," Nat. Mater., vol. 8, pp. 83-85, 2009.

[9] S. Chu and A. Majumdar, "Opportunities and challenges for a sustainable energy future," Nature, vol. 488, pp. 294-303, 2012.

[10] K. Matsubara and M. Matsuura, A Thermoelectric Application to Vehicles, ch. 52, pp. 1-10. Florida: CRC Press, 2005.

[11] X. Zheng, C. Liu, Y. Yan, and Q. Wang, "A review of thermoelectrics research-Recent developments and potentials for sustainable and renewable energy applications," Renewable Sustainable Energy Rev., vol. 32, pp. 486 503, 2014.

[12] T. Kajikawa, Thermoelectric Power Generation System Recovering Industrial Waste Heat, ch. 50. Florida: CRC Press, 2005.

[13] K.-T. Park, S.-M. Shin, A. S. Tazebay, H.-D. Um, J.-Y. Jung, S.-W. Jee, M.-W. Oh, S.-D. Park, B. Yoo, C. Yu, and J.-H. Lee, "Lossless hybridization between photovoltaic and thermoelectric devices," Sci. Rep., vol. 3, pp. -, 2013.

[14] D. Zhao and G. Tan, "A review of thermoelectric cooling: Materials, modeling and applications," Appl. Therm. Eng., vol. 66, pp. 15 - 24, 2014.

[15] X. Liu, W. X. Zhao, and L. H. Lingling, Packaging of High Power Semiconductor Lasers. New York: Spinger, 2014.

[16] S. B. Riffat and X. Ma, "Thermoelectrics: a review of present and potential applications," Appl. Therm. Eng., vol. 23, pp. 913-935, 2003. 
[17] D. Kraemer, B. Poudel, H.-P. Feng, J. C. Caylor, B. Yu, X. Yan, Y. Ma, X. Wang, D. Wang, A. Muto, K. McEnaney, M. Chiesa, Z. Ren, and G. Chen, "High-performance flat-panel solar thermoelectric generators with high thermal concentration," Nat. Mater., vol. 10, pp. 532-538, 2011.

[18] L. L. Baranowski, G. J. Snyder, and E. S. Toberer, "Concentrated solar thermoelectric generators," Energy Environ. Sci., vol. 5, pp. 9055-9067, 2012.

[19] W. Wong, D. Anderson, K. Tuttle, and R. Tew, "Status of NASA's advanced radioisotope power conversion technology research and development," in Space Technology and Applications International Forum - STAIF 2006, vol. 813 of AIP CONFERENCE PROCEEDINGS, pp. 340-347, 2006.

[20] J. Yang and T. Caillat, "Thermoelectric Materials for Space and Automotive Power Generation,” MRS Bull., vol. 31, pp. 224-229, 2006.

[21] L.-D. Zhao, V. P. Dravid, and M. G. Kanatzidis, "The panoscopic approach to high performance thermoelectrics," Energy Environ. Sci., vol. 7, pp. 251-268, 2014.

[22] H. J. Goldsmid, Introduction to Thermoelectricity. Heidelberg: Springer, 2009.

[23] T. J. Seebeck, "Ueber die magnetische Polarisation der Metalle und Erze durch Temperatur-Differenz," Ann. Phys., vol. 82, pp. 133-160, 1826. This article was first published at Abh. Akad. Wiss. Berlin, 289-346 (1820-21).

[24] E. Velmre, “Thomas Johann Seebeck (1770-1831)," Proc. Estonian Acad. Sci. Eng., vol. 13, pp. 276-282, 2007.

[25] Ørsted, Hans Chirstian, Experimenta circa efeffect conflictus electrici in acum magneticam. Hafniae, Copenhagen: Typis Schulzianis, 1820. The copy of this book can be found in URL : openlibray.org/publishers/Typis_Schulzianis.

[26] G. Magnus, "Ueber thermoelecktrische Ströme," Poggerndorffs Annalen der Physik, vol. 83, p. 469, 1851. The collection of Poggerndorffs Annalen der Physik can be found via URL :

de.wikisource.org/wiki/Annalen_der_Physikorgallica.bnf.fr.

[27] J. Martin, T. Tritt, and C. Uher, "High temperature Seebeck coefficient metrology," J. Appl. Phys., vol. 108, p. 121101, 2010.

[28] J. C. A. Peltier, "Nouvelles expansionériences sur la caloricité des courants électrique," Annalens de Chimie et de Physique, vol. 56, pp. 371-386, 1834.

[29] B. Spencer and W. R. Smith, eds., The New Werner Twentieth Century Edition of the Encyclopaedia Britannica, vol. 18. Werner Company, reviesed from 9th ed., 1907.

[30] T. D. Sparks, Oxide Thermoelectrics: The Role of Crystal Structure on Thermopower in Strongly Correlated Spinels. PhD thesis, Harvard University, Massachusetts, USA, May 2012.

[31] M. Grundmann, The Physics of Semiconductors; An introduction including Devices and Nanophysics. Heidelberg: Springer, 2006. 
[32] C. B. P. Finn, Thermal Physics. London: Chapmam \& Hall, 2nd ed., 1993.

[33] D. M. Rowe, General Principles and Basic Considerations, ch. 1, pp. 1-10. Florida: CRC Press, 2005.

[34] G. S. Nolas, J. Shaph, and H. J. Goldsmid, Thermoelectrics: Basic Principles and New Materials Developments. Springer Series in Materials Science, Heidelberg: Spinger, 2001.

[35] H. J. Goldsmid, Conversion Efficiency and Figure-of-Merit, ch. 3. Florida: CRC, 1995.

[36] N. W. Aschcroft and N. D. Mermin, Solid State Physics. California: Brooks Cole, 1976.

[37] C. Kittel, Introduction to Solid State Physics. USA: John Wiley \& Sons, Inc., 8th ed., 2005.

[38] M. Born and T. von Kármán, "Uber Schwingungen in Raumgittern," Physik. Z, vol. 13, p. 297, 1912.

[39] M. Born and K. Huang, Dynamical theory of crystal lattices. Oxford: Oxford University Press, 1964.

[40] G. K. Horton and A. A. Maradudin, Dynamical properties of solids. Dynamical Properties of Solids, North-Holland, 1974.

[41] J. M. Ziman, Principles of the Theory of Solids. Cambridge: Cambridge University Press, 2nd ed., 1979.

[42] J. M. Ziman, Electrons and Phonon: The Theory of Transport Phenomena in Solid. Oxford: Oxford University Press, 1960.

[43] F. Reif, Statistical Thermal Physics. New York: McGraw-hill book company, 1965.

[44] A. J. Minnich, "Advances in the measurement and computation of thermal phonon transport properties," J. Phys. Condens. Matter, vol. 27, p. 053202, 2015.

[45] P. G. Klemens, "Thermal Resistance due to Point Defects at High Temperatures," Phys. Rev., vol. 119, p. 507, 1960.

[46] H. Morkoç, Nitride Semiconductor Devices : Fundamentals and Applications. John Wiley \& Sons, 2013.

[47] J. Callaway, "Model for Lattice Thermal Conductivity at Low Temperatures," Phys. Rev., vol. 113, p. 1046, 1959.

[48] H. J. Goldsmid and A. W. Penn, "Boundary scattering of pphonon in solid solutions," Phys. Lett. A, vol. 27A, pp. 523-524, 1968.

[49] R. B. Wilson and D. G. Cahill, "Anisotropic failure of Fourier theory in time-domain thermoreflectance experiments," Nat. Commun., vol. 5, p. 5075, 2014. 
[50] G. A. Slack, New Materials and Performance Limits for Thermoelectric Cooling, ch. 34. Florida: CRC Press, 1995.

[51] G. S. Nolas, D. T. Morelli, and T. M. Tritt, "SKUTTERUDITES: A Phonon-Glass-Electron Crystal Approach to Advanced Thermoelectric Energy Conversion Applications," Annu. Rev. Mater. Sci., vol. 29, pp. 89-116, 1999.

[52] M. Christensen, A. B. Abrahamsen, N. B. Christensen, F. Juranyi, N. H. Andersen, K. Lefmann, J. Andreasson, C. R. H. Bahl, and B. B. Iversen, "Avoided crossing of ratter modes in thermoelectric materials," Nat. Mater., vol. 7, p. 811, 2008.

[53] C. Uher, Recent Advanced in the Development of Efficient N-Type Skutterudites, ch. 10, pp. 10-1. Florida: CRC, 2012.

[54] J. Sootsman, D. Y. Chung, and M. Kanatzidis, "New and Old Concepts in Thermoelectric Materials," Angew. Chem. Int. Ed., vol. 48, pp. 8616-8639, 2009.

[55] T. Tadano, Y. Gohda, and S. Tsuneyuki, "Impact of Rattlers on Thermal Conductivity of a Thermoelectric Clathrate: A First-Principles Study," Phys. Rev. Lett., vol. 114, p. 095501, 2015.

[56] K. Koumoto, R. Funahashi, E. Guilmeau, Y. Miyazaki, A. Weidenkaff, Y. Wang, and C. Wan, "Thermoelectric Ceramics for Energy Harvesting," J. Am. Ceram. Soc., vol. 96, pp. 1-23, 2013.

[57] L.-D. Zhao, J. He, D. Berardan, Y. Lin, J.-F. Li, C.-W. Nan, and N. Dragoe, "BiCuSeO oxyselenides: new promising thermoelectric materials," Energy Environ. Sci., vol. 7, pp. 2900-2924, 2014.

[58] Y.-L. Pei, H. Wu, D. Wu, F. Zheng, and J. He, "High Thermoelectric Performance Realized in a BiCuSeO System by Improving Carrier Mobility through 3D Modulation Doping," JACS, vol. 136, pp. 13902-13908, 2014.

[59] D. T. Morelli, J. P. Heremans, and G. A. Slack, "Estimation of the isotope effect on the lattice thermal conductivity of group IV and group III-V semiconductors," Phys. Rev. B, vol. 66, p. 195304, 2002.

[60] P. G. Klemens, "The Scattering of Low-Frequency Lattice Waves by Static Imperfections," Proc. Phys. Soc. London, Sect. A, vol. 68, p. 1113, 1955.

[61] J. Callaway and H. C. von Baeyer, "Effect of Point Imperfections on Lattice Thermal Conductivity," Phys. Rev., vol. 120, p. 1149, 1960.

[62] B. Abeles, "Lattice Thermal Conductivity of Disordered Semiconductor Alloys at High Temperatures," Phys. Rev., vol. 131, p. 1906, 1963.

[63] G. P. Srivastava, "Tuning phonon properties in thermoelectric materials," Rep. Prog. Phys., vol. 78, p. 026501, 2015.

[64] H. B. G. Casimir, "Note on the conduction of heat in crystals," Physica, vol. 5, pp. 495-500, 1938.

[65] K. E. Goodson and Y. S. Ju, "HEAT CONDUCTION IN NOVEL ELECTRONIC FILMS," Annu. Rev. Mater. Sci., vol. 29, pp. 261-293, 1999. 
[66] P. G. Klemens, “Theory of Thermal Conduction in Thin Ceramic Films," Int. J. Thermophys., vol. 22, pp. 265-275, 2001.

[67] N. Mingo, "Calculation of Si nanowire thermal conductivity using complete phonon dispersion relations," Phys. Rev. B, vol. 68, p. 113308, 2003.

[68] A. I. Persson, Y. K. Koh, D. G. Cahill, L. Samuelson, and H. Linke, "Thermal Conductance of InAs Nanowire Composites," Nano Lett., vol. 9, pp. 4484-4488, 2009.

[69] Z. Zhiyun, Z. Xuechao, and J. Guojun, "Thermally driven spin transport through a transverse-biased zigzag-edge graphene nanoribbon," J. Phys. Condens. Matter, vol. 24, p. 095302, 2012.

[70] H. Böttner, G. Chen, and R. Venkatasubramanian, "Aspects of Thin-Film Superlattice Thermoelectric Materials, Devices, and Applications," MRS Bull., vol. 31, pp. 211-217, 2006.

[71] Y. K. Koh, Y. Cao, D. G. Cahill, and D. Jena, "Heat-Transport Mechanisms in Superlattices," Adv. Funct. Mater., vol. 19, pp. 610-615, 2009.

[72] R. Venkatasubramanian, "Lattice thermal conductivtiy reduction and phonon localizationlike behavior in superlattice structures," Phys. Rev. B, vol. 61, p. 3091, 2000.

[73] K. Biswas, J. He, Q. Zhang, G. Wang, C. Uher, V. P. Dravid, and M. G. Kanatzidis, "Strained endotaxial nanostructures with high thermoelectric figure of merit," Nat. Chem., vol. 3, pp. 160-166, 2011.

[74] K. Biswas, J. He, I. D. Blum, C.-I. Wu, T. P. Hogan, D. N. Seidman, V. P. Dravid, and M. G. Kanatzidis, "High-performance bulk thermoelectrics with all-scale hierarchical architectures," Nature, vol. 489, pp. 414-418, 2012.

[75] S. I. Kim, K. H. Lee, H. A. Mun, H. S. Kim, S. W. Hwang, J. W. Roh, D. J. Yang, W. H. Shin, X. S. Li, Y. H. Lee, G. J. Snyder, and S. W. Kim, "Dense dislocation arrays embedded in grain boundaries for high-performance bulk thermoelectrics," Science, vol. 348, p. 109, 2015.

[76] F. L. Madarasz and P. G. Klemens, "Phonon scattering by dislocations in metallic alloys," Phys. Rev. B, vol. 23, p. 2553, 1981.

[77] G. D. Mahan and J. O. Sofo, "The best thermoelectric," Proc. Nat. Acad. Sci. U.S.A., vol. 93, p. 7436, 1996.

[78] Z. Fan, H.-Q. Wang, and J.-C. Zheng, "Searching for the best thermoelectrics through the optimization of transport distribution function," J. Appl. Phys., vol. 109, p. 073713, 2011.

[79] J. P. Heremans, V. Jovovic, E. S. Toberer, A. Saramat, K. Kurosaki, A. Charoenphakdee, S. Yamanaka, and G. J. Snyder, "Enhancement of thermoelectric efficiency in PbTe by distortion of the electronic density of states," Science, vol. 321, p. 554, 2008.

[80] N. F. Mott and H. Jones, The theory of the properties of metals and alloys. New York: Dover Publications, Inc., 1958. 
[81] A. Ishida, T. Yamada, T. Nakano, Y. Takano, and S. Takaoka, "Seebeck Effects and Electronic Thermal Conductivity of IV-VI Materials," Jpn. J. Appl. Phys., vol. 50, no. 3R, p. 031302, 2011.

[82] A. M. Rao, X. Ji, and T. M. Tritt, "Properties of Nanostructured One-Dimensional and Composite Thermoelectric Materials," MRS Bull., vol. 31, pp. 218-223, 2006.

[83] J. P. Heremans, B. Wiendlocha, and A. M. Chamoire, "Resonant levels in bulk thermoelectric semiconductors," Energy Environ. Sci., vol. 5, pp. 5510-5530, 2012.

[84] Y.-L. Pei and Y. Liu, "Electrical and thermal transport properties of Pb-based chalcogenides: PbTe, PbSe, and PbS," J. Alloys Compd., vol. 514, pp. 40-44, 2012.

[85] R. P. Chasmar and R. Stratton, "The Thermoelectric Figure of Merit and its Relation to Thermoelectric Generators," J. Electr. Contr., vol. 7, pp. 52-72, 1959.

[86] Y. Pei, A. D. LaLonde, H. Wang, and G. J. Snyder, "Low effective mass leading to high thermoelectric performance," Energy Environ. Sci., vol. 5, pp. 7963-7969, 2012.

[87] Y. Pei, H. Wang, and G. J. Snyder, "Band Engineering of Thermoelectric Materials," Adv. Mater., vol. 24, pp. 6125-6135, 2012.

[88] G. S. Nolas, J. Poon, and M. Kanatzidis, "Recent Developments in Bulk Thermoelectric Materials," MRS Bull., vol. 31, pp. 199-205, 2006.

[89] G. A. Slack and M. A. Hussain, "The maximum possible conversion efficiency of silicon-germanium thermoelectric generators," J. Appl. Phys., vol. 70, p. 2694, 1991.

[90] L. D. Hicks and M. S. Dresselhaus, "Effect of quantum-well structures on the the thermoelectric figure of merit," Phys. Rev. B, vol. 47, p. 12727, 1993.

[91] Y. Pei, X. Shi, A. LaLonde, H. Wang, L. Chen, and G. J. Snyder, "Convergence of electronic bands for high performance bulk thermoelectrics," Nature, vol. 473, pp. 66-69, 2011.

[92] H. Jin, C. M. Jaworski, and J. P. Heremans, "Enhancement in the figure of merit of p-type $\mathrm{Bi}_{100-x} \mathrm{Sb}_{x}$ alloys through multiple valence-band doping," Appl. Phys. Lett., vol. 101, p. 053904, 2012.

[93] W. Liu, X. Tan, K. Yin, H. Liu, X. Tang, J. Shi, Q. Zhang, and C. Uher, "Convergence of Conduction Bands as a Means of Enhancing Thermoelectric Performance of n-Type $\mathrm{Mg}_{2} \mathrm{Si}_{1-x} \mathrm{Sn}_{x}$ Solid Solutions," Phys. Rev. Lett., vol. 108, p. 166601, 2012.

[94] H. Wang, Y. Pei, A. D. LaLonde, and G. J. Snyder, "Weak electron-phonon coupling contributing to high thermoelectric performance in n-type PbSe," Proc. Natl. Acad. Sci. U.S.A., vol. 109, p. 9705, 2012. 
[95] D. M. Zayachuk, "The dominant mechaisms of charge-carrier scattering in lead telluride," Semiconductors, vol. 31, p. 173, 1997.

[96] M. Lundstrom, Fundamentals of carrier transport. Cambridge, United Kingdom: Cambridge University Press, 2nd ed., 2000.

[97] C. Jeong, R. Kim, and M. S. Lundstrom, "On the best bandstructure for thermoelectric performance: A Landauer perspective," J. Appl. Phys., vol. 111, p. 113707, 2012.

[98] G. D. Mahan, "Figure of merit for thermoelectrics," J. Appl. Phys., vol. 65, p. $1578,1989$.

[99] J. O. Sofo and G. D. Mahan, "Optimum band gap of a thermoelectric material," Phys. Rev. B, vol. 49, p. 4565, 1994.

[100] D. Vashaee and A. Shakouri, "Improved Thermoelectric Power Factor in Metal-Based Superlattices," Phys. Rev. Lett., vol. 92, p. 106103, 2004.

[101] D. Vashaee and A. Shakouri, "Electronic and thermoelectric transport in semiconductor and metallic superlattices," J. Appl. Phys., vol. 95, p. 1233, 2004.

[102] R. Venkatasubramanian, E. Siivola, T. Colpitts, and B. O'Quinn, "Thin-film thermoelectric devices with high room-temperature figures of merit," Nature, vol. 413, pp. 597-602, 2001.

[103] G. Zeng, J. M. O. Zide, W. Kim, J. E. Bowers, A. C. Gossard, Z. Bian, Y. Zhang, A. Shakouri, S. L. Singer, and A. Majumdar, "Cross-plane Seebeck coefficient of ErAs:InGaAs/InGaAlAs superlattices,” J. Appl. Phys., vol. 101, p. 034502 , 2007.

[104] J. M. Zide, D. O. Klenov, S. Stemmer, A. C. Gossard, G. Zeng, J. E. Bowers, D. Vashaee, and A. Shakouri, "Thermoelectric power factor in semiconductors with buried epitaxial semimetallic nanoparticles," Appl. Phys. Lett., vol. 87, pp. 112102-3, 2005.

[105] J. M. O. Zide, D. Vashaee, Z. X. Bian, G. Zeng, J. E. Bowers, A. Shakouri, and A. C. Gossard, "Demonstration of electron filtering to increase the Seebeck coefficient in $\mathrm{In}_{0.53} \mathrm{GaAs} / \mathrm{In}_{0.53} \mathrm{Ga}_{0.28} \mathrm{Al}_{0.19}$ As superlattices," Phys. Rev. B, vol. 74, p. 205335, 2006.

[106] M. Zebarjadi, Z. Bian, R. Singh, A. Shakouri, R. Wortman, V. Rawat, and T. Sands, "Thermoelectric Transport in a ZrN/ScN Superlattice," J. Electron. Mater., vol. 38, pp. 960-963, 2009.

[107] R. Dingle, H. L. Störmer, A. C. Gossard, and W. Wiegmann, "Electron mobilities in modulation-doped semiconductor heterojunction superlattices," Appl. Phys. Lett., vol. 33, p. 665, 1978.

[108] T. Mimura, S. Hiyamizu, T. Fujii, and K. Nanbu, "A New Field-Effect Transistor with Selectively Doped GaAs/n-Al $\mathrm{Ga}_{1-x}$ As Heterojunctions," Jpn. J. Appl. Phys., vol. 19, p. L225, 1980. 
[109] M. Zebarjadi, G. Joshi, G. Zhu, B. Yu, A. Minnich, Y. Lan, X. Wang, M. Dresselhaus, Z. Ren, and G. Chen, "Power Factor Enhancement by Modulation Doping in Bulk Nanocomposites," Nano Lett., vol. 11, pp. 2225-2230, 2011.

[110] Q. Hou, B. Gu, Y. Chen, Y. He, and J. Sun, "Enhancement of the thermoelectric power factor of $\mathrm{MnSi}_{1.7}$ film by modulation doping of $\mathrm{Al}$ and Cu," Appl. Phys. A, vol. 114, pp. 943-949, 2014.

[111] D. Wu, Y. Pei, Z. Wang, H. Wu, L. Huang, L.-D. Zhao, and J. He, "Significantly Enhanced Thermoelectric Performance in n-type Heterogeneous BiAgSeS Composites," Adv. Funct. Mater., vol. 24, pp. 7763-7771, 2014.

[112] S. V. Faleev and F. Léonard, "Theory of enhancement of thermoelectric properties of materials with nanoinclusions," Phys. Rev. B, vol. 77, p. 214304 , 2008.

[113] J. Yang, H.-L. Yip, and A. K.-Y. Jen, "Rational Design of Advanced Thermoelectric Materials," Adv. Energy Mater., vol. 3, pp. 549-565, 2013.

[114] A. J. Minnich, M. S. Dresselhaus, Z. F. Ren, and G. Chen, "Bulk nanostructured thermoelectric materials: current research and future prospects," Energy Environ. Sci., vol. 2, pp. 466-479, 2009.

[115] S. Mukerjee and J. E. Moore, "Doping dependence of thermopower and thermoelectricity in strongly correlated materials," Appl. Phys. Lett., vol. 90, p. 112107, 2007.

[116] L. E. Toth, Transition metal carbies and nitrides. New York-London: Academic Press, 1971.

[117] D. Gall, I. Petrov, N. Hellgren, L. Hultman, J. E. Sundgren, and J. E. Greene, "Growth of poly- and single-crystal ScN on $\mathrm{MgO}(001)$ : Role of low-energy $\mathrm{N}_{2}^{+}$ irradiation in determining texture, microstructure evolution, and mechanical properties," J. Appl. Phys., vol. 84, p. 6034, 1998.

[118] V. Rawat, Y. K. Koh, D. G. Cahill, and T. D. Sands, "Thermal conductivity of (Zr,W)N/ScN," J. Appl. Phys., vol. 105, p. 024909, 2009.

[119] S. W. King, R. F. Davis, and R. J. Nemanich, "Gas source molecular beam epitaxy of scandium nitride on silicon carbide and gallium nitride surfaces," $J$. Vac. Sci. Technol., A, vol. 32, p. 061504, 2014.

[120] J. P. Dismukes, W. M. Yim, J. J. Tietjen, and R. E. Novak, "Vapor deposition of semiconducting mononitrides of Sc, Y, and the rare earth elements," J. Cryst. Growth, vol. 9, p. 295, 1971.

[121] J. P. Dismukes, W. M. Yim, J. J. Tietjen, and R. E. Novak, "Vapor deposition of semiconducting mononitrides of scandium, yttrium, and the rare- earth elements," R.C.A. Review, vol. 31, pp. 680-691, 1970.

[122] H. A. Al-Brithen, A. R. Smith, and D. Gall, "Surface and bulk electronic structure of $\mathrm{ScN}(001)$ investigated by scanning tunneling microscopy/spectroscopy and optical absorption spectroscopy," Phys. Rev. B, vol. 70, p. 045303, 2004. 
[123] H. A. H. Al-Brithen, E. M. Trifan, D. C. Ingram, A. R. Smith, and D. Gall, "Phase stability, nitrogen vacancies, growth mode, and surface structure of ScN(001) under Sc-rich conditions," J. Cryst. Growth, vol. 242, pp. 345-354, 2002.

[124] J. P. Dismukes, W. M. Yim, and V. S. Ban, "Epitaxial growth and properties of semiconducting ScN," J. Cryst. Growth, vol. 13-14, pp. 365-370, 1972.

[125] D. Gall, M. Städele, K. Järrendahl, I. Petrov, P. Desjardins, R. T. Haasch, T. Y. Lee, and J. E. Greene, "Electronic structure of ScN determined using optical spectroscopy, photoemission, and ab initio calculations," Phys. Rev. B, vol. 63, p. 125119, 2001.

[126] G. Travaglini, F. Marabelli, R. Monnier, E. Kaldis, and P. Wachter, "Electronic structure of ScN," Phys. Rev. B, vol. 34, p. 3876, 1986.

[127] W. R. L. Lambrecht, "Electronic structure and optical spectra of the semimetal ScAs and of the indirect-band-gap semiconductors ScN and GdN," Phys. Rev. $B$, vol. 62, p. 13538, 2000.

[128] C. Stampfl, W. Mannstadt, R. Asahi, and A. J. Freeman, "Electronic structure and physical properties of early transition metal mononitrides:

Density-functional theory LDA, GGA, and screened-exchange LDA FLAPW calculations," Phys. Rev. B, vol. 63, p. 155106, 2001.

[129] M. A. Moram, Z. H. Barber, and C. J. Humphreys, "The effect of oxygen incorporation in sputtered scandium nitride films," Thin Solid Films, vol. 516, pp. 8569-8572, 2008.

[130] R. Deng, B. D. Ozsdolay, P. Y. Zheng, S. V. Khare, and D. Gall, "Optical and transport measurement and first-principles determination of the $\mathrm{ScN}$ band gap," Phys. Rev. B, vol. 91, p. 045104, 2015.

[131] D. Gall, I. Petrov, P. Desjardins, and J. E. Greene, "Microstructural evolution and Poisson ratio of epitaxial $\mathrm{ScN}$ grown on $\mathrm{TiN}(001) / \mathrm{MgO}(001)$ by ultrahigh vacuum reactive magnetron sputter deposition," J. Appl. Phys., vol. 86, p. 5524, 1999.

[132] J. M. Gregoire, S. D. Kirby, G. E. Scopelianos, F. H. Lee, and R. B. van Dover, "High mobility single crystalline ScN and single-orientation epitaxial YN on sapphire via magnetron sputtering," J. Appl. Phys., vol. 104, p. 074913, 2008.

[133] H. Al-Brithen and A. R. Smith, "Molecular beam epitaxial growth of atomically smooth scandium nitride films," Appl. Phys. Lett., vol. 77, p. 2485, 2000 .

[134] M. A. Moram, T. B. Joyce, P. R. Chalker, Z. H. Barber, and C. J. Humphreys, "Microstructure of epitaxial scandium nitride films grown on silicon," Appl. Surf. Sci., vol. 252, pp. 8385-8387, 2006.

[135] Moreno-Armenta, M. G. and Soto, G., "Electronic structure of scandium nitride with nitrogen and scandium deficits," Comput. Mater. Sci., vol. 40, pp. 275-281, 2007. 
[136] H. C. Barshilia, N. Selvakumar, B. Deepthi, and K. S. Rajam, "A comparative study of reactive direct current magnetron sputtered CrAlN and $\mathrm{CrN}$ coatings," Surf. Coat. Technol., vol. 201, pp. 2193-2201, 2006.

[137] G. G. Fuentes, R. Rodriguez, J. C. Avelar-Batista, J. Housden, F. Montalá, L. J. Carreras, A. B. Cristóbal, J. J. Damborenea, and T. J. Tate, "Recent advances in the chromium nitride PVD process for forming and machining surface protection," J. Mater. Process. Technol., vol. 167, pp. 415-421, 2005.

[138] M. L. Kuruppu, G. Negrea, I. P. Ivanov, and S. L. Rohde, "Monolithic and multilayer $\mathrm{Cr} / \mathrm{CrN}, \mathrm{Cr} / \mathrm{Cr}_{2} \mathrm{~N}$, and $\mathrm{Cr}_{2} \mathrm{~N} / \mathrm{CrN}$ coatings on hard and soft substrates," J. Vac. Sci. Technol., A, vol. 16, pp. 1949-1955, 1998.

[139] T. Polcar, T. Kubart, R. Novák, L. Kopecký, and P. Široký, "Comparison of tribological behaviour of TiN, TiCN and CrN at elevated temperatures," Surf. Coat. Technol., vol. 193, pp. 192-199, 2005.

[140] T. Polcar, N. M. G. Parreira, and R. Novák, "Friction and wear behaviour of CrN coating at temperatures up to $500^{\circ}$ C," Surf. Coat. Technol., vol. 201, pp. 5228-5235, 2007.

[141] J. Lin, W. D. Sproul, and J. J. Moore, "Microstructure and properties of nanostructured thick CrN coatings," Mater. Lett., vol. 89, pp. 55-58, 2012.

[142] J. Lin, W. D. Sproul, and J. J. Moore, "Tribological behavior of thick CrN coatings deposited by modulated pulsed power magnetron sputtering," Surf. Coat. Technol., vol. 206, pp. 2474-2483, 2012.

[143] D. Gall, C.-S. Shin, T. Spila, M. Odén, M. J. H. Senna, J. E. Greene, and I. Petrov, "Growth of single-crystal $\mathrm{CrN}$ on $\mathrm{MgO}(001)$ : Effects of low-energy ion-irradiation on surface morphological evolution and physical properties," J. Appl. Phys., vol. 91, p. 3589, 2002.

[144] C. X. Quintela, J. P. Podkaminer, M. N. Luckyanova, T. R. Paudel, E. L. Thies, D. A. Hillsberry, D. A. Tenne, E. Y. Tsymbal, G. Chen, C.-B. Eom, and F. Rivadulla, "Epitaxial CrN Thin Films with High Thermoelectric Figure of Merit," Adv. Mater., p. 1500110, 2015. DOI:10.1002/adma.201500110.

[145] C. Petrogalli, L. Montesano, M. Gelfi, G. M. La Vecchia, and L. Solazzi, "Tribological and corrosion behavior of CrN coatings: Roles of substrate and deposition defects," Surf. Coat. Technol., vol. 258, pp. 878-885, 2014.

[146] J. Lin, N. Zhang, W. D. Sproul, and J. J. Moore, "A comparison of the oxidation behavior of $\mathrm{CrN}$ films deposited using continuous dc, pulsed dc and modulated pulsed power magnetron sputtering," Surf. Coat. Technol., vol. 206, pp. 3283-3290, 2012.

[147] N. Beliardouh, K. Bouzid, C. Nouveau, B. Tlili, and M. Walock, "Tribological and electrochemical performances of $\mathrm{Cr} / \mathrm{CrN}$ and $\mathrm{Cr} / \mathrm{CrN} / \mathrm{CrAlN}$ multilayer coatings deposited by RF magnetron sputtering," Tribology International, vol. 82, pp. 443-452, 2015.

[148] P. M. Fabis, R. A. Cooke, and S. McDonough, "The influence of microstructure on stress state of sputter deposited chromium nitride films," J. Vac. Sci. Technol., A, vol. 8, pp. 3819-3826, 1990. 
[149] P. S. Herle, M. Hegde, N. Vasathacharya, S. Philip, M. R. Rao, and T. Sripathi, "Synthesis of TiN, VN, and CrN from Ammonolysis of $\mathrm{TiS}_{2}, \mathrm{VS}_{2}$, and $\mathrm{Cr}_{2} \mathrm{~S}_{3}$," J. Solid State Chem., vol. 134, pp. 120-127, 1997.

[150] O. Jankovský, D. Sedmidubský, v. Huber, P. Šimek, and Z. Sofer, "Synthesis, magnetic and transport properties of oxygen-free CrN ceramics," J. Eur. Ceram. Soc., vol. 34, pp. 4131-4136, 2014.

[151] C. X. Quintela, F. Rivadulla, and J. Rivas, "Thermoelectric properties of stoichiometric and hole-doped CrN," Appl. Phys. Lett., vol. 94, p. 152103, 2009.

[152] C. X. Quintela, B. Rodrguez-González, and F. Rivadulla, "Thermoelectric properties of heavy-element doped CrN," Appl. Phys. Lett., vol. 104, p. 022103, 2014.

[153] S. Wang, X. Yu, J. Zhang, M. Chen, J. Zhu, L. Wang, D. He, Z. Lin, R. Zhang, K. Leinenweber, and Y. Zhao, "Experimental invalidation of phase-transition-induced elastic softening in CrN," Phys. Rev. B, vol. 86, p. 064111, 2012.

[154] L. M. Corliss, N. Elliott, and J. M. Hastings, "Antiferromagnetic Structure of CrN," Phys. Rev., vol. 117, p. 929, 1960.

[155] A. Filippetti, W. E. Pickett, and B. M. Klein, "Competition between magnetic and structural transitions in CrN," Phys. Rev. B, vol. 59, p. 7043, 1999.

[156] A. Filippetti and N. A. Hill, "Magnetic Stress as a Driving Force of Structural Distortions: The Case of CrN," Phys. Rev. Lett., vol. 85, p. 5166, 2000.

[157] C. Constantin, M. B. Haider, D. Ingram, and A. R. Smith, "Metal/semiconductor phase transition in chromium nitride(001) grown by rf-plasma-assisted molecular-beam epitaxy," Appl. Phys. Lett., vol. 85, p. 6371, 2004.

[158] P. Hones, M. Diserens, R. Sanjinés, and F. Lévy, "Electronic structure and mechanical properties of hard coatings from the chromium-tungsten nitride system," J. Vac. Sci. Technol., B, vol. 18, pp. 2851-2856, 2000.

[159] K. Inumaru, K. Koyama, N. Imo-oka, and S. Yamanaka, "Controlling the structural transition at the Néel point of CrN epitaxial thin films using epitaxial growth," Phys. Rev. B, vol. 75, p. 054416, 2007.

[160] X. Y. Zhang, J. S. Chawla, B. M. Howe, and D. Gall, "Variable-range hopping conduction in epitaxial CrN(001)," Phys. Rev. B, vol. 83, p. 165205, 2011.

[161] F. Rivadulla, M. Banobre-Lopez, C. X. Quintela, A. Pineiro, V. Pardo, D. Baldomir, M. A. Lopez-Quintela, J. Rivas, C. A. Ramos, H. Salva, J.-S. Zhou, and J. B. Goodenough, "Reduction of the bulk modulus at high pressure in CrN," Nat. Mater., vol. 8, pp. 947-951, 2009.

[162] H. Willmann, P. Mayrhofer, P. A. Persson, A. Reiter, L. Hultman, and C. Mitterer, "Thermal stability of Al-Cr-N hard coatings," Scripta Mater., vol. 54, pp. 1847-1851, 2006. 
[163] J. Birch, T. Joelsson, F. Eriksson, N. Ghafoor, and L. Hultman, "Single crystal $\mathrm{CrN} / \mathrm{ScN}$ superlattice soft X-ray mirrors: Epitaxial growth, structure, and properties," Thin Solid Films, vol. 514, pp. 10-19, 2006.

[164] H. Willmann, M. Beckers, J. Birch, P. Mayrhofer, C. Mitterer, and L. Hultman, "Epitaxial growth of Al-Cr-N thin films on MgO(111)," Thin Solid Films, vol. 517, pp. 598-602, 2008.

[165] F. Rovere, D. Music, S. Ershov, M. to Baben, H.-G. Fuss, P. H. Mayrhofer, and J. M. Schneider, "Experimental and computational study on the phase stability of Al-containing cubic transition metal nitrides," J. Phys. D: Appl. Phys., vol. 43, p. 035302, 2010.

[166] F. Lomello, M. A. P. Yazdi, F. Sanchette, F. Schuster, M. Tabarant, and A. Billard, "Temperature dependence of the residual stresses and mechanical properties in TiN/CrN nanolayered coatings processed by cathodic arc deposition," Surf. Coat. Technol., vol. 238, pp. 216-222, 2014.

[167] S.-F. Chen, Y.-C. Kuo, C.-J. Wang, S.-H. Huang, J.-W. Lee, Y.-C. Chan, H.-W. Chen, J.-G. Duh, and T.-E. Hsieh, "The effect of $\mathrm{Cr} / \mathrm{Zr}$ chemical composition ratios on the mechanical properties of $\mathrm{CrN} / \mathrm{ZrN}$ multilayered coatings deposited by cathodic arc deposition system," Surf. Coat. Technol., vol. 231, pp. 247-252, 2013.

[168] S. Khamseh, M. Nose, T. Kawabata, T. Nagae, K. Matsuda, and S. Ikeno, "A comparative study of CrAlN films synthesized by dc and pulsed dc reactive magnetron facing target sputtering system with different pulse frequencies," J. Alloys Compd., vol. 508, pp. 191-195, 2010.

[169] D. Tytko, P.-P. Choi, and D. Raabe, "Thermal dissolution mechanisms of AlN/CrN hard coating superlattices studied by atom probe tomography and transmission electron microscopy," Acta Mater., vol. 85, pp. 32 - 41, 2015.

[170] D. Gall, C.-S. Shin, R. T. Haasch, I. Petrov, and J. E. Greene, "Band gap in epitaxial NaCl-structure CrN(001) layers," J. Appl. Phys., vol. 91, p. 5882, 2002.

[171] X. Y. Zhang, J. S. Chawla, R. P. Deng, and D. Gall, "Epitaxial suppression of the metal-insulator transition in CrN," Phys. Rev. B, vol. 84, p. 073101, 2011.

[172] A. S. Botana, F. Tran, V. Pardo, D. Baldomir, and P. Blaha, "Electronic structure of CrN: A comparison between different exchange correlation potentials," Phys. Rev. B, vol. 85, p. 235118, 2012.

[173] A. Herwadkar and W. R. L. Lambrecht, "Electronic structure of CrN: A borderline Mott insulator," Phys. Rev. B, vol. 79, p. 035125, 2009.

[174] B. Alling, T. Marten, and I. A. Abrikosov, "Effect of magnetic disorder and strong electron correlations on the thermodynamics of CrN," Phys. Rev. B, vol. 82, p. 184430, 2010.

[175] B. Alling, T. Marten, and I. A. Abrikosov, "Questionable collapse of the bulk modulus in CrN," Nat. Mater., vol. 9, pp. 283-284, 2010. 
[176] X. Y. Zhang and D. Gall, "CrN electronic structure and vibrational modes: An optical analysis," Phys. Rev. B, vol. 82, p. 045116, 2010.

[177] P. Tomeš, D. Logvinovich, J. Hejtmánek, M. H. Aguirre, and A. Weidenkaff, "Magnetic influence on thermoelectric properties of $\mathrm{CrO}_{0.1} \mathrm{~N}_{0.9}$," Acta Mater., vol. 59, pp. $1134-1140,2011$.

[178] B. Alling, Configurational and Magnetic Interactions in Multicomponent System. $\mathrm{PhD}$ thesis, Linköping University, Linköping, Sweden, 2010.

[179] M. Ekholm, Theoretical descriptions of complex magnetism in transition metals and their alloys. PhD thesis, Linköping University, Linköping, Sweden, 2012.

[180] O. Hellman, Thermal properties of materials from first principles. $\mathrm{PhD}$ thesis, Linköping University, Linköping, Sweden, 2012.

[181] P. Steneteg, Development of molecular dynamics methodology for simulations of hard material. PhD thesis, Linköping University, Linköping, Sweden, 2012.

[182] F. Ercolessi, "A molecular dynamics primer," lecture note, International School for Advanced Studies, Triste, Italy, June 1997. URL : www.sissa.it/furio.

[183] M. Born and R. Oppenheimer, "Zur Quantentheorie der Molekeln," Ann. Phys., vol. 389, pp. 457-484, 1927.

[184] M. R. Martin, Electronic Structure: Basic Theory and Practical Methods. Cambridge: Cambridge University press, 2004.

[185] D. R. Hartree, "The Wave Mechanics of an Atom with a Non-Coulomb Central Field. Part I. Theory and Methods," Math. Proc. Cambridge Philos. Soc., vol. 24, pp. 89-110, 1928.

[186] D. R. Hartree, "The Wave Mechanics of an Atom with a Non-Coulomb Central Field. Part II. Some Results and Discussion," Math. Proc. Cambridge Philos. Soc., vol. 24, pp. 111-132, 1928.

[187] D. R. Hartree, "The Wave Mechanics of an Atom with a non-Coulomb Central Field. Part III. Term Values and Intensities in Series in Optical Spectra," Math. Proc. Cambridge Philos. Soc., vol. 24, pp. 426-437, 1928.

[188] V. Fock, "Näherungsmethode zur Lösung des quantenmechanischen Mehrkörperproblems," Z. Phys. A: Hadrons Nucl., vol. 61, pp. 126-148, 1930.

[189] P. Hohenberg and W. Kohn, "Inhomogeneous Electron Gas," Phys. Rev., vol. 136, p. B864, 1964.

[190] W. Kohn and L. J. Sham, "Self-Consistent Equations Including Exchange and Correlation Effects," Phys. Rev., vol. 140, p. A1133, 1965.

[191] J. Harris, "Adiabatic-connection approach to Kohn-Sham theory," Phys. Rev. A, vol. 29, p. 1648, 1984.

[192] A. D. Becke, "Density-functional thermochemistry. I. The effect of the exchange-only gradient correction," J. Chem. Phys., vol. 96, pp. 2155-2160, 1992. 
[193] J. P. Perdew and A. Zunger, "Self-interaction correction to density-functional approximations for many-electron systems," Phys. Rev. B, vol. 23, p. 5048, 1981.

[194] A. D. Becke, "Density-functional exchange-energy approximation with correct asymptotic behavior," Phys. Rev. A, vol. 38, p. 3098, 1988.

[195] V. I. Anisimov, J. Zaanen, and O. K. Andersen, "Band theory and Mott insulators: Hubbard U instead of Stoner I," Phys. Rev. B, vol. 44, p. 943, 1991.

[196] V. I. Anisimov, I. V. Solovyev, and M. A. Korotin, "Density-functional theory and NiO photoemission spectra," Phys. Rev. B, vol. 48, p. 16929, 1993.

[197] E. R. Ylvisaker, W. E. Pickett, and K. Koepernik, "Anisotropy and magnetism in the LSDA + U method," Phys. Rev. B, vol. 79, p. 035103, 2009.

[198] A. D. Becke, "A new mixing of Hartree-Fock and local density-functional theories," J. Chem. Phys., vol. 98, pp. 1372-1377, 1993.

[199] A. D. Becke, "Density-functional thermochemistry. III. The role of exact exchange," J. Chem. Phys., vol. 98, pp. 5648-5652, 1993.

[200] J. Heyd, G. E. Scuseria, and M. Ernzerhof, "Hybrid functionals based on a screened Coulomb potential," J. Chem. Phys., vol. 118, pp. 8207-8215, 2003.

[201] D. M. Ceperley and B. J. Alder, "Ground State of the Electron Gas by a Stochastic Method," Phys. Rev. Lett., vol. 45, p. 566, 1980.

[202] A. D. Becke, "Density-functional thermochemistry. II. The effect of the Perdew-Wang generalized-gradient correlation correction," J. Chem. Phys., vol. 97, pp. 9173-9177, 1992.

[203] E. Finazzi, C. Di Valentin, G. Pacchioni, and A. Selloni, "Excess electron states in reduced bulk anatase TiO2: Comparison of standard GGA, GGA+U, and hybrid DFT calculations,” J. Chem. Phys., vol. 129, p. 154113, 2008.

[204] J. P. Perdew and Y. Wang, "Accurate and simple analytic representation of the electron-gas correlation energy," Phys. Rev. B, vol. 45, p. 13244, 1992.

[205] J. P. Perdew, K. Burke, and M. Ernzerhof, "Generalized Gradient Approximation Made Simple,” Phys. Rev. Lett., vol. 77, p. 3865, 1996.

[206] C. Höglund, J. Bareño, J. Birch, B. Alling, Z. Czigány, and L. Hultman, "Cubic $\mathrm{Sc}_{1-x} \mathrm{Al}_{x} \mathrm{~N}$ soild solution thin films deposited by reactive magnetron sputter epitaxy onto ScN(111)," J. Appl. Phys., vol. 105, p. 113517, 2009.

[207] B. Alling, A. Karimi, and I. A. Abrikosov, "Electronic origin of the isostructural decomposition in cubic $\mathrm{M}_{1-x} \mathrm{Al}_{x} \mathrm{~N}(\mathrm{M}=\mathrm{Ti}, \mathrm{Cr}, \mathrm{Sc}, \mathrm{Hf})$ : A first-principles study," Surf. Coat. Technol., vol. 203, pp. 883-886, 2008.

[208] C. Stampfl and C. G. Van de Walle, "Density-functional calculations for III-V nitrides using the local-density approximation and the generalized gradient approximation," Phys. Rev. B, vol. 59, p. 5521, 1999.

[209] H. Xiao, J. Tahir-Kheli, and W. A. Goddard, "Accurate Band Gaps for Semiconductors from Density Functional Theory," J. Phys. Chem. Lett., vol. 2, pp. 212-217, 2011. 
[210] P. Giannozzi, "Numerical Methods in Electronic Structure," lecture note, University of Udine, Udine, 2010. URL : www.fisica.uniud.it/ giannozz/Corsi/MetNum/metnum.html.

[211] D. A. Andersson, S. I. Simak, B. Johansson, I. A. Abrikosov, and N. V. Skorodumova, "Modeling of $\mathrm{CeO}_{2}, \mathrm{Ce}_{2} \mathrm{O}_{3}$, and $\mathrm{CeO}_{2-x}$ in the $\mathrm{LDA}+\mathrm{U}$ formalism," Phys. Rev. B, vol. 75, p. 035109, 2007.

[212] V. I. Anisimov and Y. Izyumov, Electronic Structure of Strongly Correlated Materials. Springer Series in Solid-State Sciences, Springer, 2010.

[213] B. Himmetoglu, A. Floris, S. de Gironcoli, and M. Cococcioni, "Hubbard-corrected DFT energy functionals: The LDA+U description of correlated systems," Int. J. Quantum Chem., vol. 114, pp. 14-49, 2014.

[214] A. G. Petukhov, I. I. Mazin, L. Chioncel, and A. I. Lichtenstein, "Correlated metals and the LDA +U method," Phys. Rev. B, vol. 67, p. 153106, 2003.

[215] S. L. Dudarev, S. Y. Savrasov, C. J. Humphreys, and A. P. Sutton, "Electron-energy-loss spectra and the structural stability of nickel oxide: An LSDA+U study," Phys. Rev. B, vol. 57, pp. 1505-1509, 1998.

[216] C. Adamo and V. Barone, "Toward reliable density functional methods without adjustable parameters: The PBE0 model," J. Chem. Phys., vol. 110, pp. 6158-6170, 1999.

[217] M. Ernzerhof, J. P. Perdew, and K. Burke, "Coupling-constant dependence of atomization energies," Int. J. Quantum Chem., vol. 64, pp. 285-295, 1997.

[218] J. P. Perdew, M. Ernzerhof, and K. Burke, "Rationale for mixing exact exchange with density functional approximations," J. Chem. Phys., vol. 105, pp. 9982-9985, 1996.

[219] J. Paier, M. Marsman, K. Hummer, G. Kresse, I. C. Gerber, and J. G. Angyan, "Screened hybrid density functionals applied to solids," J. Chem. Phys., vol. 124, pp. 154709-13, 2006.

[220] J. Heyd, J. E. Peralta, G. E. Scuseria, and R. L. Martin, "Energy band gaps and lattice parameters evaluated with the Heyd-Scuseria-Ernzerhof screened hybrid functional," J. Chem. Phys., vol. 123, pp. 174101-8, 2005.

[221] C. Rödl, F. Fuchs, J. Furthmüller, and F. Bechstedt, "Quasiparticle band structures of the antiferromagnetic transition-metal oxides $\mathrm{MnO}, \mathrm{FeO}, \mathrm{CoO}$, and NiO," Phys. Rev. B, vol. 79, p. 235114, 2009.

[222] V. L. Chevrier, S. P. Ong, R. Armiento, M. K. Y. Chan, and G. Ceder, "Hybrid density functional calculations of redox potentials and formation energies of transition metal compounds," Phys. Rev. B, vol. 82, p. 075122, 2010.

[223] P. E. Blöchl, "Projector augmented-wave method," Phys. Rev. B, vol. 50, p. 17953, 1994.

[224] D. Poster and E. K.E., Phase Transformations in Metals and Alloys. Florida: CRC Press, 2nd ed., 2004. 
[225] A. McNaught and A. Wilkinson, IUPAC. Compendium of Chemical Terminology, 2nd ed, (the "Gold Book"). Oxford: Blackwell Scientific Publications, 1997.

[226] D. Dye, "Microstructure and Properties of Materials," lecture note, Imperial College, London, 2014. URL : dyedavid.com/mse104/.

[227] P. Haasen, Physical Metallurgy. London: Cambridge University Press, 3rd ed., 1996.

[228] P. H. Mayrhofer, A. Hörling, L. Karlsson, J. Sjölén, T. Larsson, C. Mitterer, and L. Hultman, "Self-organized nanostructures in the Ti-Al-N system," Appl. Phys. Lett., vol. 83, p. 2049, 2003.

[229] A. Thore, M. Dahlqvist, B. Alling, and J. Rosén, “Temperature dependent phase stability of nanolaminated ternaries from first-principles calculations," Comput. Mater. Sci., vol. 91, pp. 251-257, 2014.

[230] A. V. Ruban and I. A. Abrikosov, "Configurational thermodynamics of alloys from first principles: effective cluster interactions," Rep. Prog. Phys., vol. 71, no. 4, p. 046501, 2008.

[231] J. Sanchez, F. Ducastelle, and D. Gratias, "Generalized cluster description of multicomponent systems," Physica A, vol. 128, p. 334, 1984.

[232] J. M. Sanchez, "Cluster expansions and the configurational energy of alloys," Phys. Rev. B, vol. 48, p. 14013, 1993.

[233] A. Zunger, S.-H. Wei, L. G. Ferreira, and J. E. Bernard, "Special quasirandom structures," Phys. Rev. Lett., vol. 65, p. 353, 1990.

[234] M. Born and K. Huang, Dynamical theory of crystal lattices. Oxford: Clarendon Press, 1954.

[235] D. Marx and J. Hutter, Modern Methods and Algorithms of Quantum Chemistry, vol. 1 of NIC Series. Jülich: John von Neumann Institute for Computing, 2000.

[236] D. Marx and J. Hutter, Ab initio molecular dynamics: basic theory and advanced methods. Cambridge University Press, 2009.

[237] R. Petrenko and J. Meller, "Molecular Dynamics," in Encyclopedia of Life Sciences, John Wiley \& Sons, Ltd, 2010.

[238] F. H. Stillinger and T. A. Weber, "Computer simulation of local order in condensed phases of silicon," Phys. Rev. B, vol. 31, p. 5262, 1985.

[239] J. Tersoff, "New empirical approach for the structure and energy of covalent systems," Phys. Rev. B, vol. 37, p. 6991, 1988.

[240] R. Feynman, "Forces in Molecules," Phys. Rev., vol. 56, p. 340, 1939.

[241] L. Verlet, "Computer "Experiments" on Classical Fluids. I. Thermodynamical Properties of Lennard-Jones Molecules," Phys. Rev., vol. 159, p. 98, 1967.

[242] C. R. de Oliveira and W. T., "Ergodic hypothesis in classical statistical mechanics," Revista Brasileira de Ensino de Física, vol. 29, pp. 189-201, 2007. 
[243] P. Hünenberger, "Thermostat Algorithms for Molecular Dynamics Simulations," in Advanced Computer Simulation (C. Dr. Holm and K. Prof. Dr. Kremer, eds.), vol. 173 of Advances in Polymer Science, pp. 105-149, Springer Berlin Heidelberg, 2005.

[244] S. Nosé, "Constant temperature molecular dynamics methods," Prog. Theor. Phys. Supp., vol. 103, pp. 1-46, 1991.

[245] H. C. Andersen, "Molecular dynamics simulations at constant pressure and/or temperature," J. Chem. Phys., vol. 72, pp. 2384-2393, 1980.

[246] M. P. Allen and D. J. Tildesley, Computer simulation of liquids. Oxford science publications, Clarendon Press, 1989.

[247] S. Nosé, "A molecular dynamics method for simulations in the canonical ensemble," Mol. Phys., vol. 52, pp. 255-268, 1984.

[248] W. Hoover, "Canonical dynamics: Equilibrium phase-space distributions," Phys. Rev. A, vol. 31, p. 1695, 1985.

[249] J. G. Kirkwood, "Statistical Mechanics of Fluid Mixtures," J. Chem. Phys., vol. 3, p. 300, 1935.

[250] D. West and S. Estreicher, "First-Principles Calculations of Vibrational Lifetimes and Decay Channels: Hydrogen-Related Modes in Si," Phys. Rev. Lett., vol. 96, p. 115504, 2006.

[251] S. Baroni, P. Giannozzi, and E. Isaev, "Density-Functional Perturbation Theory for Quasi-Harmonic Calculations," Rev. Mineral. Geochem., vol. 71, pp. 39-57, 2010.

[252] B. Fultz, "Vibrational thermodynamics of materials," Prog. Mater Sci., vol. 55, p. 247, 2010.

[253] A. Togo, L. Chaput, I. Tanaka, and G. Hug, "First-principles phonon calculations of thermal expansion in $\mathrm{Ti}_{3} \mathrm{SiC}_{2}, \mathrm{Ti}_{3} \mathrm{AlC}_{2}$, and $\mathrm{Ti}_{3} \mathrm{GeC}_{2}$," Phys. Rev. B, vol. 81, p. 174301, 2010.

[254] K. Parlinski, Z. Q. Li, and Y. Kawazoe, "First-Principles Determination of the Soft Mode in Cubic $\mathrm{ZrO}_{2}$," Phys. Rev. Lett., vol. 78, p. 4063, 1997.

[255] A. Togo, F. Oba, and I. Tanaka, "First-principles calculations of the ferroelastic transition between rutile-type and $\mathrm{CaCl}_{2}$-type $\mathrm{SiO}_{2}$ at high pressures," Phys. Rev. B, vol. 78, p. 134106, 2008.

[256] S. Baroni, P. Giannozzi, and A. Testa, "Green's-function approach to linear response in solids," Phys. Rev. Lett., vol. 58, p. 1861, 1987.

[257] P. Giannozzi, S. de Gironcoli, P. Pavone, and S. Baroni, "Ab initio calculation of phonon dispersions in semiconductors," Phys. Rev. B, vol. 43, p. 7231, 1991.

[258] S. Baroni, S. de Gironcoli, A. Dal Corso, and P. Giannozzi, "Phonons and related crystal properties from density-functional perturbation theory," Rev. Mod. Phys., vol. 73, pp. 515-562, 2001. 
[259] L. Dubrovinsky, N. Dubrovinskaia, O. Narygina, I. Kantor, A. Kuznetzov, V. B. Prakapenka, L. Vitos, B. Johansson, A. S. Mikhaylushkin, S. I. Simak, and I. A. Abrikosov, "Body-Centered Cubic Iron-Nickel Alloy in Earth's Core," Science, vol. 316, p. 1880, 2007.

[260] O. Hellman, I. A. Abrikosov, and S. I. Simak, "Lattice dynamics of anharmonic solids from first principles," Phys. Rev. B, vol. 84, p. 180301, 2011.

[261] O. Hellman and I. A. Abrikosov, "Temperature-dependent effective third-order interatomic force constants from first principles," Phys. Rev. B, vol. 88, p. 144301, 2013.

[262] M. L. Klein and G. K. Horton, “The rise of self-consistent phonon theory," J. Low Temp. Phys., vol. 9, pp. 151-166, 1972.

[263] M. Born, "Die Gültigkeitsgrenze der Theorie der idealen Kristalle und ihre Überwindung.," Festschr. Akad. Wiss. Göttingen, Math.-Phys. Kl., vol. 1, pp. 1-16, 1951.

[264] N. Gillis, N. Werthamer, and T. Koehler, "Properties of Crystalline Argon and Neon in the Self-Consistent Phonon Approximation," Phys. Rev., vol. 165, p. 951, 1968.

[265] P. Souvatzis, O. Eriksson, M. I. Katsnelson, and S. Rudin, "Entropy Driven Stabilization of Energetically Unstable Crystal Structures Explained from First Principles Theory," Phys. Rev. Lett., vol. 100, p. 95901, 2008.

[266] L. Chaput, A. Togo, I. Tanaka, and G. Hug, "Phonon-phonon interactions in transition metals," Phys. Rev. B, vol. 84, p. 094302, 2011.

[267] O. Hellman and D. A. Broido, "Phonon thermal transport in $\mathrm{Bi}_{2} \mathrm{Te}_{3}$ from first principles," Phys. Rev. B, vol. 90, p. 134309, 2014.

[268] D. J. Hooton, "Anharmonische Gitterschwingungen und die lineare Kette," $Z$. Angew. Phys., vol. 142, pp. 42-57, 1955.

[269] A. A. Maradudin and S. Vosko, "Symmetry Properties of the Normal Vibrations of a Crystal," Rev. Mod. Phys., vol. 40, pp. 1-37, 1968.

[270] O. Hellman, P. Steneteg, I. A. Abrikosov, and S. I. Simak, "Temperature dependent effective potential method for accurate free energy calculations of solids," Phys. Rev. B, vol. 87, p. 104111, 2013.

[271] B. Grabowski, L. Ismer, T. Hickel, and J. Neugebauer, "Ab initio up to the melting point: Anharmonicity and vacancies in aluminum," Phys. Rev. B, vol. 79, p. 134106, 2009.

[272] M. Omini and A. Sparavigna, "An iterative approach to the phonon Boltzmann equation in the theory of thermal conductivity," Physica B, vol. 212, p. 101, 1995.

[273] A. Ward, D. A. Broido, D. A. Stewart, and G. Deinzer, "Ab initio theory of the lattice thermal conductivity in diamond," Phys. Rev. B, vol. 80, p. 125203, 2009. 
[274] J. R. Yates, X. Wang, D. Vanderbilt, and I. Souza, "Spectral and Fermi surface properties from Wannier interpolation," Phys. Rev. B, vol. 75, p. 195121, 2007.

[275] P. Lambin and J. P. Vigneron, "Computation of crystal Green's functions in the complex-energy plane with the use of the analytical tetrahedron method," Phys. Rev. B, vol. 29, p. 3430, 1984.

[276] S. Lee, K. Esfarjani, J. Mendoza, M. S. Dresselhaus, and G. Chen, "Lattice thermal conductivity of $\mathrm{Bi}, \mathrm{Sb}$, and Bi-Sb alloy from first principles," Phys. Rev. B, vol. 89, p. 085206, 2014.

[277] M. Ohring, The materials science of Thin Films. California: Academic Press, 2nd ed., 1992.

[278] W. Grove, "On the Electro-Chemical Polarity of Gases," Philos. Trans. R. Soc. London, Ser. A, vol. 142, pp. 87-101, 1852.

[279] I. Langmuir, "Osillations in Ionized gases," Proc. Nat. Acad. Sci. U.S.A., vol. 14, p. 627, 1928.

[280] M. Lieberman and A. Lichtenberg, Principles of Palsma Discharges and Materials Processing. New York: John Wiley \& Sons, 2nd ed., 2005.

[281] J. Vossen and W. Kern, Thin Film Processes II. New York: Academic Press, 1991.

[282] P. Eklund, Multifunctional nanostructured Ti-Si-C thin films. PhD thesis, Linköping University, Linköping, Sweden, 2007.

[283] R. F. Bunshah, Handboork of Deposition Tehchnologies for Films and Coatings; Science, Technology, and Applications. New Jersey: Noyes Publications, 2nd ed., 1994.

[284] K. Seshan, Handbook of Thin-Film Deposition Processes Processes and Techniques. New York: Noyes Publications/William Andrew Pulishing, 2nd ed., 2001.

[285] P. E. J. Flewitt and R. K. Wild, Physical Methods for Materials Characterization. Philadelphia: Institute of Physics Publishing, 2nd ed., 2003.

[286] M. Birkholz, Thin Film Analysis by X-Ray Scattering. Weinheim: Wiley-VCH, 2006.

[287] D. B. Williams and C. B. Carter, Transmission Electron Microscopy. New York: Spinger, 2009.

[288] D. K. Schroder, Semiconductor Material and Device Characterization. New York: John Wiley \& Sons Inc., 3rd ed., 2006.

[289] R. B. Roberts, F. Righini, and R. C. Compton, "Absolute scale of thermoelectricity III,” Philos. Mag. B, vol. 52, pp. 1147-1163, 1985.

[290] G. A. Antonelli, B. Perrin, B. C. Daly, and D. G. Cahill, "Characterization of Mechanical and Thermal Properties Using Ultrafast Optical Metrology," MRS Bull., vol. 31, pp. 607-613, 2006. 
[291] D. G. Cahill, "Analysis of heat flow in layered structures for time-domain thermoreflectance," Rev. Sci. Instrum., vol. 75, p. 5119, 2004.

[292] D. G. Cahill, P. V. Braun, G. Chen, D. R. Clarke, S. Fan, K. E. Goodson, P. Keblinski, W. P. King, G. D. Mahan, A. Majumdar, H. J. Maris, S. R. Phillpot, E. Pop, and L. Shi, "Nanoscale thermal transport. II. 2003-2012," Appl. Phys. Rev., vol. 1, p. 011305, 2014.

[293] A. Schmidt, R. Cheaito, and M. Chiesa, "A frequency-domain themoreflectance method for the characterization of thermal properties," Rev. Sci. Instrum., vol. 80, p. 094901, 2009.

[294] D. G. Cahill, W. K. Ford, K. E. Goodson, G. D. Mahan, A. Majumdar, H. J. Maris, R. Merlin, and S. R. Phillpot, "Nanoscale thermal transport," J. Appl. Phys., vol. 93, p. 793, 2003.

[295] Y. Deng, H.-m. Liang, Y. Wang, Z.-w. Zhang, M. Tan, and J.-1. Cui, "Growth and transport properties of oriented bismuth telluride films," J. Alloys Compd., vol. 509, pp. 5683-5687, 2011.

[296] C. Höglund, B. Alling, J. Birch, M. Beckers, P. O. A. Persson, C. Baehtz, Z. Czigány, J. Jensen, and L. Hultman, "Effects of volume mismatch and electronic structure on the decomposition of ScAlN and TiAlN solid solutions," Phys. Rev. B, vol. 81, p. 224101, 2010. 
Papers 



\section{Papers}

The articles associated with this thesis have been removed for copyright reasons. For more details about these see:

http://urn.kb.se/resolve?urn=urn:nbn:se:liu:diva-117760 